\title{
AN ANALYTICAL, CONTROL-ORIENTED STATE SPACE MODEL FOR A PEM FUEL CELL SYSTEM
}

\author{
THÈSE No 3445 (2005) \\ PRÉSENTÉE À LA FACULTÉ SCIENCES ET TECHNIQUES DE L'INGÉNIEUR \\ Institut des sciences de l'énergie \\ SECTION DE GÉNIE ÉLECTRIQUE ET ÉLECTRONIQUE \\ ÉCOLE POLYTECHNIQUE FÉDÉRALE DE LAUSANNE \\ POUR L'OBTENTION DU GRADE DE DOCTEUR ÈS SCIENCES
}

PAR

Félix GASSER

ingénieur mécanicien diplômé EPF de nationalité suisse et originaire d'Eich (LU)

acceptée sur proposition du jury:

Prof. A.-Ch Rufer, directeur de thèse

Prof. D. Favrat, rapporteur

Dr K. Rajashekara, rapporteur

Dr J. Van Herle, rapporteur

Prof. A. Wokaun, rapporteur 



\section{Preface}

The results presented in this thesis have been elaborated during my time at the "Laboratoire d'Electronique Industrielle" directed by Prof. A. Rufer. The project was financed through support from the Swiss Federal Office of Energy. I would like to take this opportunity to express my gratitude to everyone who contributed to making it all possible.

My sincere thanks go to Prof. Rufer for accepting me in his lab and for the confidence he placed in me throughout this project.

I would like to thank the members of the jury, Prof. D. Favrat, Dr. J. Van herle, Dr. K. Rajashekera and Prof. A. Wokaun, particularly Dr. Rajashekara and Dr. Van herle for their discussions and insightful comments during the writing of the manuscript.

I am equally indebted to Dr. F. Büchi and A. Tsukada along with the whole fuel cell team at PSI who have introduced me to PEM fuel cells. Working with them during my two months at PSI has been a very enriching experience.

I am very grateful to Dr. Pascal Favre from the "Services Industriels de la Ville de Lausanne" for his generous loan of a $300 \mathrm{~W}$ fuel cell system upon which the first version of the test bench was based.

My thanks go to Olivier Räss, Nora Ludewig, Jean-Luc Arend and Toufann Chaudhuri who have worked with me on various diploma and semester projects. Many of their contributions have become part of this thesis.

I would like to thank all my colleagues from the Laboratoire d'Electronique Industrielle. Their friendship and support have provided the both relaxing and stimulating environment that allowed this work to come to fruition. My thanks go to Fabienne Vionnet for her assistance with all things administrative and to Roberto Zoia for providing a reliable, state of the art computing infrastructure.

Special thanks go to Yves Birbaum for his assistance in developing the test bench. I would also like to express my gratitude to Jean-Paul Barben, 
Jean-Paul Brugger, Pierre-André Pognant, Jean-Pierre Grobet and Karim Collomb who have spent a great deal of time manufacturing and installing custom-made parts of the experimental setup.

I would like to express my sincerest thanks to my dedicated and critical team of editors, my wife Neslihan, Philippe Barrade, Christian Blanc and Kaushik Rajashekara. Their valuable and insightful comments have made this thesis into what it is. Needless to say that all remaining errors are entirely mine.

Finally, I am infinitely grateful to all my friends and family, especially my wife Neslihan, who have provided much needed moral support and encouragement throughout this project. 


\section{Summary}

If fuel cell technology - with its inherent benefits of high efficiency and low emissions - is to be used in decentralised power sources, in mobile or transportation applications, the systems have to be able to adapt to fast load changes and varying operating conditions.

In order to achieve such performance, the balance of plant systems typically governed by an on-board system controller - need to dynamically supply the fuel cell stack with reactant gases at the right flow rates, pressures and humidities while keeping the fuel cell at its correct operating temperature. Since best overall system performance is achieved by using model-based controllers, an appropriate model is required to implement such controllers.

This thesis provides a control-oriented state space model for a PEM fuel cell system. The model describes the effects of a user interaction with any of the balance of plant actuators on overall system performance.

The system model is elaborated in a two-step process. In a first step, an analytical, steady state, cell-averaged, isothermal fuel cell stack model is developed. The model predicts the fuel cell voltage and membrane water content as a function of the stack's operating conditions - i.e. reactant flow rates, pressures and humidities as well as cell temperature. It provides an analytical expression to the overall water transport within the fuel cell stack.

In the second step, dynamic state space models are developed for the balance of plant systems. They link the effects of the auxiliary systems' actuators to the evolution of the operating conditions for the fuel cell stack.

In the context of this thesis, state space models for a non-pressurised air supply subsystem, for a recirculating, pressurised hydrogen supply subsystem and for a liquid cooled thermal management subsystem are elaborated.

A dedicated fuel cell test bench has been developed that was used to experimentally validate the proposed models. 



\section{Résumé}

Les pile à combustible sont aujourd'hui pressenties pour la production d'énergie décentralisée, pour des applications mobiles ou encore dans le secteur des transports. Elles possèdent en effet les avantages inhérents de rendements élevés et de faibles émissions. Cependant, pour de telles applications, elles doivent être capable de suivre des changements de charge rapides et de s'adapter à des conditions de fonctionnement variables.

Pour arriver à des telles performances, les systèmes auxiliaires - typiquement contrôlés par des régulateurs intégrés - doivent dynamiquement alimenter la pile avec les réactants aux bons débits, pressions et humidités, tout en maintenant une température idéale. Comme les meilleures performances sont obtenues en utilisant des régulateurs à base de modèles, il faut disposer d'un modèle approprié du système pour implémenter de tels régulateurs.

Cette thèse développe un modèle pour un système pile à combustible PEM. Le modèle décrit les effets d'une interaction de l'utilisateur avec les systèmes auxiliaires sur les performances de la pile à combustible.

Le modèle est établi en deux parties. Dans une première partie, un modèle analytique, isotherme, moyenné sur la cellule et en régime établi est développé. Le modèle prédit la tension de cellule et le contenu en eau de la membrane en fonction des conditions des réactants d'une part, et de la température d'autre part.

Dans une deuxième partie, des modèles d'état dynamiques sont développés pour les systèmes auxiliaires. Ils font le lien entre les actuateurs des systèmes auxiliaires et l'évolution des conditions de fonctionnement de la pile. Dans le contexte de cette thèse, des modèles d'état sont établis pour un système d'approvisionnement en air non pressurisé, pour un système d'approvisionnement en hydrogène pressurisé à re-circulation et pour un système de contrôle de température.

Un banc de test dédié a été développé lors de cette thèse. Les modèles établis y ont été vérifiés expérimentalement. 



\section{Zusammenfassung}

Damit Brennstoffzellen, mit ihren inhärenten Vorteilen von gutem Wirkungsgrad und geringen Emissionen, als dezentrale Kraftwerke, in mobilen Anwendungen oder im Transportsektor zum Einsatz kommen können, müssen sie sich schnell ändernden Last- und Betriebsbedingungen anpassen können.

Um solche Eigenschaften zu erreichen müssen die Hilfsaggregate, typischerweise durch integrierte Regler gesteuert, die Brennstoffzelle dynamisch mit der richtigen Menge an korrekt konditionierten Reaktionsgasen versorgen und das System bei der optimalen Temperatur halten. Da beste Resultate durch modell-basierte Regler erreicht werden, muss ein geeignetes Modell zur Verfügung stehen um solche Regler zu implementieren.

Diese Arbeit stellt ein regelungsorientiertes Zustandsmodell eines PEM Brennstoffzellensystems bereit. Das Modell beschreibt die Auswirkungen einer Interaktion mit den Hilfsaggregaten auf die Systemleistung.

Das Modell wird in zwei Stufen entwickelt. In einer ersten Stufe wird ein analytisches, eingeschwungenes, zellengemitteltes, isothermes Brennstoffzellenmodell entwickelt. Das Modell berechnet die Zellspannung und den Wassergehalt der Membran als Funktion der Betriebsbedingungen, das heisst Zelltemperatur und Massenstrom, Druck und Feuchte der Reaktionsgase. Eine analytische Beschreibung des Wassertransports in der Zelle wird erarbeitet.

In der zweiten Stufe werden dynamische Zustandsmodelle für die Hilfsaggregate entwickelt. Diese Modelle formen das Bindeglied zwischen den Stellorganen der Hilfsaggregate und den daraus resultierenden Betriebsbedingungen des Stapels. In dieser Arbeit werden Zustandsmodelle für eine nicht mit Druck beaufschlagte Luftspeisung, eine mit Druck beaufschlagte, rezirkulierende Wasserstoffspeisung sowie für eine wassergekühlte Temperaturregelung erarbeitet.

Ein Brennstoffzellenteststand wurde entwickelt und die erarbeiteten Modelle darauf experimentell verifiziert. 



\section{Contents}

Preface $\quad$ i

Summary iii

Résumé $v$

Zusammenfassung vii

Notations $\quad$ xv

1. Introduction $\quad 1$

1.1. The Global Picture . . . . . . . . . . . . . . . 2

1.2. Fuel Cells and The Hydrogen Economy . . . . . . . . . . . 2

1.3. Introduction to Fuel Cells . . . . . . . . . . . . . 3

1.4. The Operating Principle of Fuel Cells . . . . . . . . . . . 6

1.5. Motivation of the Present Work . . . . . . . . . . . . . 9

1.6. Literature Survey . . . . . . . . . . . . . . . . . 10

1.7. Contributions ................. 13

1.8. Structure of this Document ............. . 14

2. Fuel Cell Stack Model 17

2.1. Introduction . . . . . . . . . . . . . . . . . 18

2.2. Fuel Cell Equilibrium Voltage . . . . . . . . . . . . . 19

2.2.1. Mixed Potential . . . . . . . . . . . . 20

2.3. Fuel Cell Losses . . . . . . . . . . . . . . . . . 21

2.3.1. Activation Overpotential . . . . . . . . . . 22

2.3.2. Diffusion Overpotential . . . . . . . . . . . . 24

2.3.3. Electronic Ohmic Overpotential . . . . . . . . . 25

2.3.4. Ionic Overpotential . . . . . . . . . . 26

2.4. Gas Transport .................. 31 
2.4.1. Description of Reactant Supply . . . . . . . . . . . 32

2.4.2. Gas Channel Concentration Averaging . . . . . . . . 33

2.4.3. Gas Transport in the Gas Diffusion Layer . . . . . . 39

2.5. Water Transport . . . . . . . . . . . . . . . . 43

2.5.1. Water Transfer Coefficient $\alpha$. . . . . . . . . 44

2.5.2. Average Gas Channel Water Concentration . . . . . 45

2.5.3. Water Transport within the GDL . . . . . . . . . 49

2.5.4. Water Transport within the Membrane . . . . . . 50

2.5.5. Iterative Approaches to Overall Water Transport . . 55

2.5.6. Analytical Expression for the Overall Water Transport 58

2.6. Overall Fuel Cell Stack Model . . . . . . . . . . . . . . . . 59

2.7. Summary and Outlook . . . . . . . . . . . . 60

3. Balance of Plant Models 63

3.1. Introduction . . . . . . . . . . . . . . . . . . . 64

3.2. System Description . . . . . . . . . . . . . . . . . 64

3.3. Air Supply Subsystem Model . . . . . . . . . . . . 66

3.3.1. Air Compressor . . . . . . . . . . . 67

3.3.2. Pressure Dynamics . . . . . . . . . . . . . 68

3.3.3. Overall Air Supply Subsystem Model . . . . . . . . 69

3.3.4. Humidifier . . . . . . . . . . . . . . . . . 69

3.3.5. Backpressure Regulator ............ . 70

3.3.6. Summary ................. . . . . 71

3.4. Hydrogen Supply Subsystem Model . . . . . . . . . . . . . . 72

3.4.1. Hydrogen Recirculation Pump . . . . . . . . . . 73

3.4.2. Hydrogen Pressure Regulator . . . . . . . . . . . . . 74

3.4.3. Hydrogen Pressure Dynamics . . . . . . . . . . . 75

3.4.4. Summary ................ . . 76

3.5. Thermal Management Subsystem Model . . . . . . . . . . . 77

3.5.1. Cooling Pump .............. . . 78

3.5.2. Thermal Fuel Cell Model . . . . . . . . . . . 79

3.5.3. Cooling Liquid Reservoir . . . . . . . . . . . . . 81

3.5.4. Liquid-Liquid Heat Exchanger . . . . . . . . . . . 82

3.5.5. Liquid-Air Heat Exchanger . . . . . . . . . . . . . 85

3.5.6. Overall Cooling Circuit . . . . . . . . . . . 85

3.5.7. State Space Representation of the Cooling Circuit . 86

3.5.8. State Space Averaged Overall Cooling Circuit . . . . 90

3.5.9. Summary ................ . . 91 
3.6. Electronic Power Converters . . . . . . . . . . . . . . . . . 91

3.7. Overall Balance of Plant Model . . . . . . . . . . . . . . . 92

3.8. Summary . . . . . . . . . . . . . . . . 93

4. Design and Description of a Dedicated Test Bench 95

4.1. Introduction . . . . . . . . . . . . . . . . 96

4.2. Description of the FC stack . . . . . . . . . . . . 96

4.3. Description of the Test Bench . . . . . . . . . . . . . . 97

4.3.1. Air Supply Subsystem . . . . . . . . . . . . 100

4.3.2. Hydrogen Supply Subsystem . . . . . . . . . . . 102

4.3.3. Thermal Management Subsystem . . . . . . . . . . 106

4.4. Data Acquisition and Control . . . . . . . . . . . . . 109

4.4.1. Data Acquisition and Control Hardware . . . . . . . 110

4.4.2. Data Acquisition and Control Software . . . . . . . . 114

4.5. Summary . . . . . . . . . . . . . . . . 118

5. Experimental Results 121

5.1. Introduction . . . . . . . . . . . . . . . . . . . 122

5.2. Fuel Cell Stack . . . . . . . . . . . . . . . . . . 122

5.2.1. Model Validation on Literature Data . . . . . . . . . 124

5.2.2. Fuel Cell Parameter Identification . . . . . . . . . . 128

5.2.3. Fuel Cell Stack Model Validation . . . . . . . . . . . 132

5.2.4. Summary . . . . . . . . . . . . . . 134

5.3. Air Supply Subsystem . . . . . . . . . . . . . 135

5.3.1. Parameter Identification . . . . . . . . . . . 135

5.3.2. Model Validation . . . . . . . . . . . . . . 139

5.3.3. Summary . . . . . . . . . . . . . . . . 142

5.4. Hydrogen Supply Subsystem . . . . . . . . . . . . . . 143

5.4.1. Parameter Identification . . . . . . . . . . . . . . . 143

5.4.2. Model Validation . . . . . . . . . . . . . . . 144

5.4.3. Pressure Dynamics in the Hydrogen Supply Manifold 146

5.4.4. Summary . . . . . . . . . . . . . . . 148

5.5. Thermal Management Subsystem . . . . . . . . . . . . . 148

5.5.1. Parameter Identification . . . . . . . . . . . . . . . 148

5.5.2. Model Validation . . . . . . . . . . . . . 156

5.5.3. Summary . . . . . . . . . . . . . 159

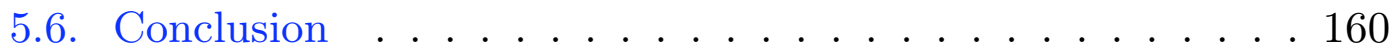


6. Conclusions and Future Work 163

6.1. Summary . . . . . . . . . . . . . . . . 164

6.2. Discussion . . . . . . . . . . . . . . 165

6.3. Future Work . . . . . . . . . . . . . . . . . 168

A. Derivation of Fuel Cell Performance Equations 171

A.1. Derivation of the Nernst equation . . . . . . . . . . . . . . . 172

A.2. Derivation of the Butler-Volmer Equation . . . . . . . . . . 173

A.3. Limiting Current Density . . . . . . . . . . . . . . . . 176

A.4. Theoretical Ionic Membrane Conductivity . . . . . . . . . . 177

B. Definition of Mass Transfer Processes and Parameters 181

B.1. Air Composition . . . . . . . . . . . . . . . . . . . 182

B.2. Gas Solubility in Water . . . . . . . . . . . . . . 182

B.3. Sherwood Type Mass Transfer . . . . . . . . . . . . . . . . 183

B.4. Diffusion . . . . . . . . . . . . . . . . . . . . . . 184

B.4.1. Binary Diffusion Coefficients . . . . . . . 185

B.4.2. Mixture Diffusion Coefficients . . . . . . . . . . 186

B.4.3. Diffusion in Porous Media . . . . . . . . . . 187

C. Analytical Solution for Water Transfer 189

C.1. Analytical Water Transport Solution . . . . . . . . . . . . . 190

C.2. Cubic Formula . . . . . . . . . . . . . . . . . . 193

C.2.1. General Solution . . . . . . . . . . . . . 193

C.2.2. Solution for Real Roots . . . . . . . . . . . . 194

$\begin{array}{lr}\text { D. State Space Notation } & 197\end{array}$

D.1. Introduction . . . . . . . . . . . . . . . . . . . . 198

D.2. Representation . . . . . . . . . . . . . . . 198

D.3. Discretisation . . . . . . . . . . . . . . . . . 199

D.4. Linearisation . . . . . . . . . . . . . . 200

D.5. Controllability and Observability . . . . . . . . . 202

D.6. State Space Averaging . . . . . . . . . . . . . . . 202

E. Matlab Implementation 205

E.1. Introduction . . . . . . . . . . . . . . . . 206

E.2. Data Structure .. . . . . . . . . . . . . . 206

E.3. Constants .................... 209

xii 
$\begin{array}{lr}\text { List of Figures } & \mathbf{2 1 5}\end{array}$

$\begin{array}{lr}\text { List of Tables } & 221\end{array}$

$\begin{array}{lr}\text { Bibliography } & 223\end{array}$

$\begin{array}{lr}\text { Curriculum Vitae } & 231\end{array}$ 



\section{Notations}

\section{Abbreviations}

$\begin{array}{ll}A D L & \text { Anode Gas Diffusion Layer } \\ A G C & \text { Anode Gas Channel } \\ B I P & \text { Bipolar Plate } \\ B O P & \text { Balance of Plant } \\ B R & \text { Backpressure Regulator } \\ C A N & \text { Controller Area Network } \\ C A T & \text { Catalyst Layer } \\ C C & \text { Cooling Circuit } \\ C D L & \text { Cathode Gas Diffusion Layer } \\ C G C & \text { Cathode Gas Channel } \\ C L & \text { Cooling Liquid } \\ C O & \text { Air Compressor } \\ C P & \text { Cooling Liquid Pump } \\ C V & \text { Chill Water Valve } \\ C W & \text { Chill Water } \\ D V & \text { Discharge Valve } \\ E H & \text { Exit Heater } \\ E I S & \text { Electrochemical Impedance Spectroscopy } \\ E P F L & \text { Ecole Polytechnique Fédérale de Lausanne } \\ E W & \text { Equivalent Weight: equivalent dry membrane weight } \\ & \text { per mol proton transport sites } \\ F C & \text { Fuel Cell } \\ G C & \text { Gas Channel } \\ G D L & \text { Gas Diffusion Layer } \\ G P I B & \text { General Purpose Instrumentation Bus, also known as } \\ H H V & \text { IEEE-488 } \\ H O R & \text { Higher Heating Value } \\ & \end{array}$




$\begin{array}{ll}H S & \text { Hydrogen Supply } \\ H X & \text { Heat Exchanger } \\ I V & \text { Current-Voltage } \\ L E I & \text { Laboratoire d'Electronique Industrielle } \\ L H V & \text { Lower Heating Value } \\ M E A & \text { Membrane Electrode Assembly } \\ P E & \text { Polymer Electrolyte } \\ P E F C & \text { Polymer Electrolyte Fuel Cell } \\ P E M & \text { Proton Exchange Membrane } \\ P S I & \text { Paul Scherrer Institute } \\ R T D & \text { Resistance Temperature Detectors } \\ P T F E & \text { Polytetrafluoroethylene (Teflon) } \\ O R R & \text { Oxygen Reduction Reaction } \\ R E G & \text { Region } \\ H P & \text { Hydrogen Recirculation Pump } \\ R V & \text { Cooling Liquid Reservoir } \\ T T L & \text { Transistor-Transistor Locic }\end{array}$

\section{Roman Symbols}

$\begin{array}{llc}a & \text { Absolute pressure (used in bar(a)) } & - \\ a_{i} & \text { Activity of species i }\left(a_{i}=p_{i} / p\right) & - \\ A & \text { Surface area } & \mathrm{m}^{2} \\ A_{s} & \text { Heat exchange surface area } & \mathrm{m}^{2} \\ c_{i} & \text { Concentration of species i } & \mathrm{mol} / \mathrm{m}^{3} \\ c_{p} & \text { Specific heat } & \mathrm{J} / \mathrm{mol} \\ d & \text { Diameter } & \mathrm{m} \\ d & \text { Duty cycle ratio } & - \\ D_{A B} & \text { Binary diffusivity (diffusion coefficient) of } \\ & \text { species A in species B } & \mathrm{m}^{2} / \mathrm{s} \\ D_{A G} & \text { Binary diffusivity (diffusion coefficient) of } \\ & \text { species A in gas mixture G } & \mathrm{m}^{2} / \mathrm{s} \\ D_{i, R E G} & \text { Effective diffusivity (diffusion coefficient) of } \\ & \text { species i in region REG } & \mathrm{m}^{2} / \mathrm{s} \\ E & \text { Cell voltage } & \mathrm{V} \\ \bar{E} & \text { Average cell voltage } & \mathrm{V}\end{array}$




\begin{tabular}{|c|c|c|}
\hline$E_{0}$ & Nernst potential & $V$ \\
\hline$F$ & Faraday constant & $\mathrm{C} / \mathrm{mol}$ \\
\hline$g$ & Gravitational acceleration & $m / s^{2}$ \\
\hline$g$ & Gauge (or relative) pressure (used in bar(g)) & - \\
\hline$\Delta \mathcal{G}$ & Molar Gibbs free reaction enthalpy & $J / m o l$ \\
\hline$h$ & Overall convective heat transfer coefficient & $\frac{W}{m^{2} K}$ \\
\hline$\Delta h$ & Reaction enthalpy per unit mass & $J / k g$ \\
\hline$H$ & Henry's constant for gas solubility & $\mathrm{Pa} \cdot \mathrm{m}^{3} / \mathrm{mol}$ \\
\hline$\Delta H$ & Reaction enthalpy & $J$ \\
\hline$\Delta \mathcal{H}_{H_{2}}$ & $\begin{array}{l}\text { Molar hydrogen heating value (Molar enthalpy } \\
\text { of reaction) }\end{array}$ & $\mathrm{J} / \mathrm{mol}$ \\
\hline$I$ & Current & $A$ \\
\hline$I^{\prime \prime}$ & Current density & $A / m^{2}$ \\
\hline$k$ & Conductive heat transfer coefficient & $\frac{W}{m \cdot K}$ \\
\hline$k_{\Phi}$ & Electrokinetic permeability & $\begin{array}{c}m \cdot n \\
m^{2}\end{array}$ \\
\hline$k_{D V}$ & Discharge coefficient & $\frac{P a}{s \ln m}$ \\
\hline$k_{F C}$ & Natural convection coefficient for the fuel cell & $\frac{W}{K}$ \\
\hline$k_{f}$ & Friction coefficient & $N m \frac{s}{r a d}$ \\
\hline$k_{h}$ & Hydraulic flow resistance & $\frac{P a}{s l p m}$ \\
\hline$k_{H P}$ & $\begin{array}{l}\text { Cooling pump quivalent voltage-flow rate } \\
\text { coefficient }\end{array}$ & $\frac{l p m}{V}$ \\
\hline$k_{H X}$ & $\begin{array}{l}\text { Specific heat exchanger power (for liquid-air } \\
\text { type) }\end{array}$ & $\frac{W}{s}$ \\
\hline$k_{m}$ & Speed-flow rate coefficient & $\frac{s l p m \cdot s}{r a d}$ \\
\hline$k_{p}$ & Hydraulic permeability & $\begin{array}{l}r a d \\
m^{2}\end{array}$ \\
\hline$k_{p}$ & $\begin{array}{l}\text { Pressure coefficient (relating compressor torque } \\
\text { to compression ratio) }\end{array}$ & $m^{3}$ \\
\hline$k_{R V}$ & $\begin{array}{l}\text { Natural convection coefficient for the water } \\
\text { reservoir }\end{array}$ & $\frac{W}{K}$ \\
\hline$k_{t}$ & Motor torque constant & $\frac{N m}{A}$ \\
\hline$k_{x}$ & Heat exchanger efficiency-flow rate coefficient & $\frac{\mathrm{A}}{\mathrm{kg}}$ \\
\hline$L$ & Length & $m$ \\
\hline$L$ & Inductance & $H$ \\
\hline$m$ & Mass & $k g$ \\
\hline$\dot{m}_{i}$ & Mass flow rate of species $\mathrm{i}$ & $\mathrm{kg} / \mathrm{s}$ \\
\hline $\mathcal{M}_{i}$ & Molar mass of species i & $\mathrm{kg} / \mathrm{mol}$ \\
\hline
\end{tabular}


$n \quad$ Number of moles of electrons exchanged in a reaction

$n_{i} \quad$ Number of moles of electrons exchanged in a reaction per mol of reactant $i$

$\left(n_{\mathrm{O}_{2}}=4, n_{H_{2}}=2\right.$ for the fuel cell reaction

$2 \mathrm{H}_{2}+\mathrm{O}_{2} \rightarrow \mathrm{H}_{2} \mathrm{O}$ )

$n_{\text {drag }} \quad$ Number of water molecules crossing the

membrane per proton (electroosmotic drag

coefficient)

$n_{F C} \quad$ Number of cells in a fuel cell stack

$\dot{N}_{i} \quad$ Molar flow rate of species i

$\dot{N}_{i}^{\prime \prime} \quad$ Molar flux density of species i

$\mathrm{Nu} \quad$ Nusselt number

$p \quad$ Total pressure

$p_{i} \quad$ Partial pressure of species i

$Q \quad$ Heat

$\dot{Q} \quad$ Heat flux

$\dot{Q}^{\prime \prime} \quad$ Heat flux density

$r_{m} \quad$ Membrane resistivity $r_{m}=\frac{1}{\sigma_{m}}$

$\mathcal{R} \quad$ Universal gas constant

$R \quad$ Ohmic resistance

Re Reynolds number

$s \quad$ Saturation ratio for two phase flow $\left(s=\frac{d V_{l}}{d V_{\text {void }}}\right)$

$S \quad$ Entropy

Sh Sherwood number

$t$

Time

$T \quad$ Temperature

$T_{r} \quad$ Reduced temperature $\left(T_{r}=\left(T-T_{c}\right) / T_{c}\right)$

$v \quad$ Translational speed or rate of progress

$\mathbf{u} \quad$ Input vector in state space notation

$U \quad$ Overall heat transfer coefficient

$V \quad$ Voltage

$V \quad$ Volume

$\dot{V} \quad$ Volumetric flow rate

$\mathcal{V} \quad$ Molar volume

$x \quad \mathrm{x}$-coordinate (x-y plane spans the FC

membrane)

$$
\begin{gathered}
\mathrm{mol} / \mathrm{s} \\
\mathrm{mol} /\left(\mathrm{m}^{2} s\right) \\
- \\
P a \\
P a \\
J \\
J / s \\
J /\left(m^{2} s\right) \\
\Omega \cdot m \\
J /(K \cdot m o l) \\
\Omega \\
- \\
- \\
J / K \\
- \\
s \\
K \\
K \\
m / s \\
- \\
W / m^{2} K \\
V \\
m^{3} \\
m^{3} / s \\
m^{3} / m o l \\
m \\
\end{gathered}
$$

xviii 
$\mathbf{x} \quad$ State vector in state space notation

$y \quad \mathrm{y}$-coordinate $(\mathrm{x}-\mathrm{y}$ plane spans the $\mathrm{FC}$

membrane)

y Output vector in state space notation

$m$ z-coordinate (perpendicular to the membrane)

\section{Greek Symbols}

$\beta \quad$ Reaction symmetry coefficient

$\gamma \quad$ GDL water transfer coefficient

$\gamma_{i} \quad$ Stoichiometric factor of component i

$\delta_{i} \quad$ Thickness of component i

$\epsilon \quad$ Heat exchanger efficiency

$\zeta \quad$ Exchange current density fitting

parameter

$\eta \quad$ Efficiency

$\eta \quad$ Overpotential

Ionic membrane Conductivity

$m$

$z \quad$ Compressibility factor

Stoichiometric flow rate of reactant $i$

$\mu_{i} \quad$ Viscosity of species i

$\rho_{i} \quad$ Mass density of species i (For

multicomponent mixtures, it represents

$m / s$

the density with respect to the overall

mixture volume such that $\rho_{m i x}=\sum_{i} \rho_{i}$ )

$\sigma_{m} \quad$ Membrane conductivity $\sigma_{m}=\frac{1}{r_{m}}$

$S / m$
$s$
$V$
-
-
$\mathrm{molH}_{2} \mathrm{O} / \mathrm{molSO}_{3}^{-}$
-
-
$\mathrm{rad} / \mathrm{s}$




\section{Indices}

\begin{tabular}{|c|c|}
\hline 0 & Standard conditions \\
\hline 1 & Initial value \\
\hline 2 & Final value \\
\hline$A, B, C$ & Components in gas mixture $\mathrm{G}$ \\
\hline act & Activation, mainly used for overpotential \\
\hline$a d m$ & Inlet manifold (french: admission) \\
\hline$a m b$ & Ambient conditions \\
\hline$a r$ & Arithmetic mean value \\
\hline$b$ & Backward \\
\hline$c$ & Critical \\
\hline$c$ & Cross sectional \\
\hline$c$ & Cold fluid \\
\hline conc & Concentration, mainly used for overpotential \\
\hline $\operatorname{conv}$ & Convective \\
\hline dif & Diffusive \\
\hline dis & Dissolved \\
\hline drag & (Mass transport by) electroosmotic drag \\
\hline el & Electrical \\
\hline$f$ & Forward \\
\hline$g$ & Gas phase \\
\hline$G$ & Gas or gas mixture \\
\hline$h$ & Hydraulic \\
\hline$h$ & Hot fluid \\
\hline$\imath$ & Species (i.e. $\mathrm{H}_{2} \mathrm{O}, \mathrm{O}_{2}$, etc.) \\
\hline in & Inlet \\
\hline ion & Ionic, mainly used for overpotential \\
\hline$i t$ & Isenthermal transformation \\
\hline$L$ & Air (germ. Luft) \\
\hline 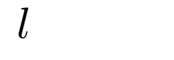 & Liquid phase \\
\hline$l m$ & Logarithmic mean value \\
\hline$m$ & Ion conductive phase or membrane \\
\hline ohm & Ohmic, mainly used for overpotential \\
\hline out & Outlet \\
\hline ref & Reference value or condition \\
\hline$S$ & Reaction surface (i.e. GDL-CAT interface) \\
\hline sat & Saturated conditions \\
\hline
\end{tabular}


$v \quad$ Water vapour

$w \quad$ Water 



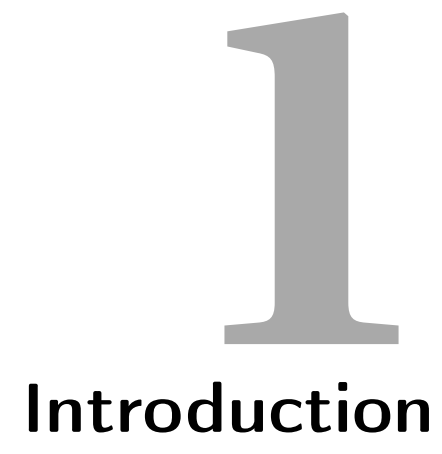




\subsection{The Global Picture}

Transforming today's non-sustainable energy usage into a globally sustainable energy economy is one of mankind's key challenges for the current century.

This transformation process encompasses a multitude of different aspects - technological, social, and economical.

It involves abandoning or changing energetically aberrant habits. Why is a mass of 1.5 tonnes displaced to transport one person for a couple of kilometres? Why is food transported around the globe when similar products are available locally? It involves energy prices reflecting a true and sustainable cost instead of excavation, transport and tax.

It involves technological advances to increase the efficiency of energy transformation and storage processes. It involves technological advances to develop more energy efficient transportation systems and housing.

While social and economical changes present a huge - and sadly so far untapped - potential in the reduction of current energy consumption, it is not enough. Technological advances are required to be able to successfully use renewables as a primary energy source. The reason lies in the stochastic nature of many renewable energy sources (i.e wind or solar power) as well as their limited availability.

Technological advances therefore need to provide solutions to the following two problems:

1. Efficient Energy Conversion

2. Energy Storage

\subsection{Fuel Cells and The Hydrogen Economy}

Fuel Cell (FC) technology with its inherent benefits of high efficiency almost twice that of a traditional internal combustion engine - and low emissions addresses efficient energy conversion. Additionally, its fuel of choice, hydrogen, provides a solution to the storage problem, allowing energy from renewable, fluctuating sources to be stored and transported until it is being used.

While hydrogen can be used as a means of storing energy [1], this only makes sense for renewable energy that could otherwise not be used. As a matter of fact, the hydrogen production-storage-usage cycle efficiency is rather low $[2,3]$. 


\subsection{Introduction to Fuel Cells}

Fuel Cells are electrochemical energy converters. They can be regarded as black-boxes (see Figure 1.1) converting chemical energy contained in a fuel directly into electrical energy while generating heat and water as by-products.

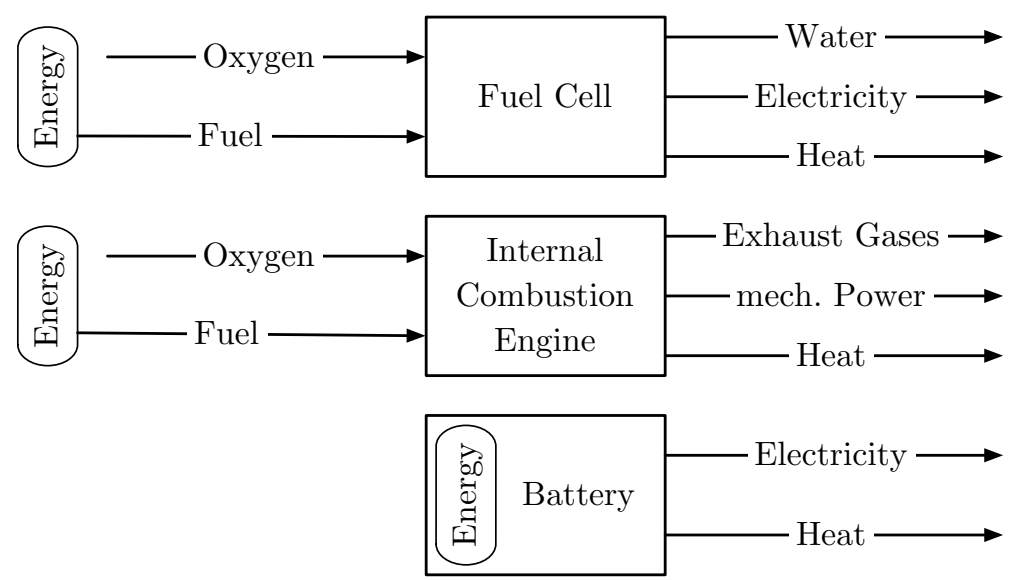

Figure 1.1: Schematic representation of different energy (battery, fuel cell and internal combustion engine) converters in the form of black-boxes.

The basic mechanism underlying this conversion is the same as the one for batteries. The primary difference being that the battery contains the reactants (i.e. fuel and oxidant) that generate electricity whereas those reactants need to be supplied externally to the fuel cell. In other words, a battery needs to be thrown away or recharged once those reactants are depleted while the fuel cell can be refuelled more easily and quickly by either refilling the tank with fuel (hydrogen) or replacing the fuel reservoir.

In this respect they are comparable to internal combustion engines (ICE) which, when provided with fuel and air, generate mechanical power with heat and exhaust gases as byproducts.

The main advantages of fuel cells with respect to traditional energy converters are:

- A high conversion efficiency. Moreover, that efficiency increases with diminishing load, a very interesting characteristic for the transportation sector where part load operation is the rule and ICEs run at reduced efficiency in low load conditions (see Figure 1.2). 


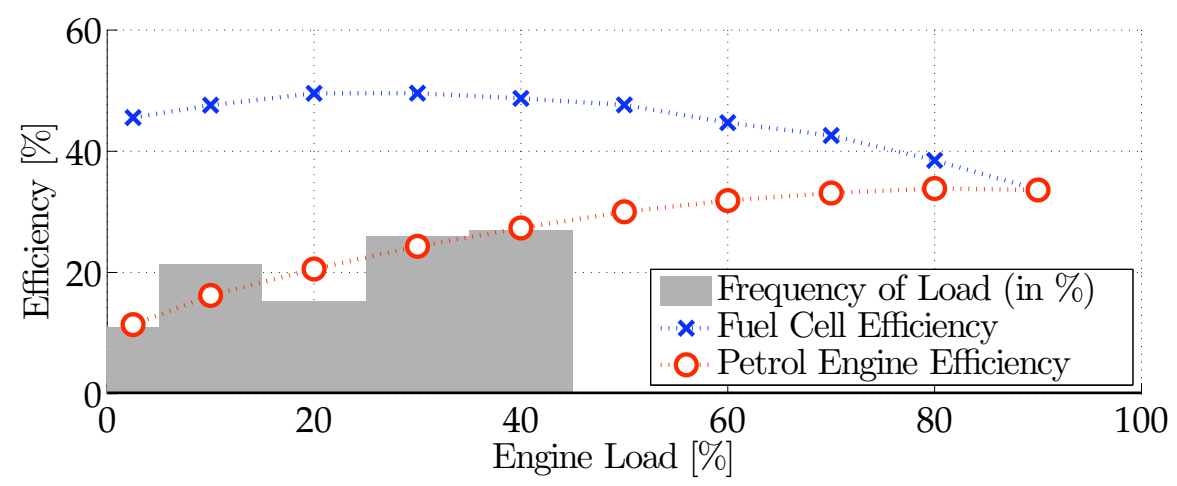

Figure 1.2: Typical Internal Combustion Engine and Fuel Cell efficiencies as a function of engine load. The bar-graph depicts the percentage at which the corresponding engine load occurs in the European ECE-15 driving cycle. Data from [4].

- Very low emissions. The actual emission level depends on the fuel - for cells fed by pure hydrogen, true zero-emission performance is achieved since the only reaction product is water. Even if natural gas or petrol is used as a fuel through a reforming process, $\mathrm{CO}_{2}$ emissions will be lower than a comparable ICE due to the fuel cell's higher efficiency. Additionally, no toxic nitrogen oxides $\left(N O_{x}\right)$ are generated.

- Low noise levels. Since the electrochemical reaction is a conversion process that requires no moving parts, operation of the fuel cell is completely silent. A fuel cell system, with all the necessary systems for cooling, power conversion and air and fuel supply will emit some noise, mainly due to the air compressor.

- System scalability. Due to their construction, fuel cell systems are modular power generators. Efficient systems can be built for power levels from several Watts to several Mega Watts.

The discovery of the fuel cell is generally attributed to Sir William R. Grove who depicted the first useful fuel cell in his article On the Gas Voltaic Battery - Voltaic Action of Phosphorus, Sulphur and Hydrocarbons [5] published in 1845. With the ongoing development of the already successful steam engine and the later discovery of the internal combustion 
engine and its adaptation for the transport sector, fuel cells did not see any industrial development until well into the 20th century.

Since then, different types of fuel cells have been developed. They are typically classified by either their operating temperature or the type of electrolyte. Table 1.1 gives an overview of the main classes of fuel cells with their associated fuels, operating temperatures and electrolyte types.

\begin{tabular}{lcccc}
\hline $\begin{array}{l}\text { Fuel Cell } \\
\text { Type }\end{array}$ & Electrolyte & $\begin{array}{c}\text { Charge } \\
\text { Carrier }\end{array}$ & Temperature & Fuel \\
\hline PEMFC & solid & $H^{+}$ & $50-90{ }^{\circ} \mathrm{C}$ & $\mathrm{H}_{2}$ \\
DMFC & solid & $\mathrm{H}^{+}$ & $50-90{ }^{\circ} \mathrm{C}$ & $\mathrm{CH}_{3} \mathrm{OH}$ \\
AFC & liquid & $\mathrm{OH}^{-}$ & $60-250^{\circ} \mathrm{C}$ & $\mathrm{H}_{2}$ \\
PAFC & liquid & $\mathrm{H}^{+}$ & $160-250{ }^{\circ} \mathrm{C}$ & $\mathrm{H}_{2}$ \\
MCFC & liquid & $\mathrm{CO}_{3}^{2-}$ & $\approx 650^{\circ} \mathrm{C}$ & $\mathrm{H}_{2}, \mathrm{CO}, \mathrm{CH}_{4}$ \\
SOFC & solid & $\mathrm{O}^{2-}$ & $750-1000^{\circ} \mathrm{C}$ & $\mathrm{H}_{2}, \mathrm{CO}, \mathrm{CH}_{4}$ \\
\hline
\end{tabular}

Table 1.1: Comparison of different fuel cells and their operating characteristics. Data from [6].

Proton Exchange Membrane Fuel Cells (PEMFC) or Polymer Electrolyte Fuel Cells (PEFC) are based on a solid polymer electrolyte. Fast startup times, low temperature operation and high power densities make them an easy to use technology especially for portable or transport applications. $C O$ poisons the catalyst and the hydrogen fuel has to be very pure. Because the polymer membrane has to be kept well humidified for good proton conduction, water management is one of the critical aspects of successfully running a PEMFC.

Direct Methanol Fuel Cells (DMFC) are similar in construction to PEM fuel cells. Since liquid methanol can be used as a fuel, no external fuel processing is required and high energy storage densities can be achieved. Unfortunately, the polymer membrane is not impermeable to liquid methanol and the resulting fuel crossover reduces overall system efficiency.

Alkaline Fuel Cells (AFC) are based on a liquid, concentrated $K O H$ electrolyte. AFCs can operate with non-precious metal catalysts (typically nickel) and therefore have a cost advantage over other types 
of fuel cells. The use of a liquid electrolyte requires an additional electrolyte re-circulation system. Unfortunately, $\mathrm{CO}_{2}$ is a poison for the liquid electrolyte and needs to be scrubbed from process air. Typically, the use of AFCs has been limited to niche applications such as military and space applications.

Phosphoric Acid Fuel Cells (PAFC) are based on a liquid acid electrolyte. Due to their higher operating temperature, they are less sensitive to $C O$ impurities in the fuel and water management is less of an issue. Additionally, they exhibit excellent long term stability. Their relatively long start-up times and low power densities limit their application to stationary power or co-generation plants.

Molten Carbonate Fuel Cells (MCFC) are based on a liquid molten carbonate electrolyte and generally exhibit very high conversion efficiencies. A high operating temperature allows direct use of non noble catalysts along with direct internal processing of fuels such as natural gas. Relatively long start-up times and low power densities again limit their application to stationary power or co-generation plants.

Solid Oxide Fuel Cells (SOFC) are based on a solid oxide electrolyte conducting oxygen $O^{2-}$ ions. As with the MCFC, the high operating temperature translates into non-noble catalysts, direct internal hydrocarbon fuel processing and high quality waste heat that can be utilised in combined-cycle power plants. Additionally, high power densities along with high efficiencies can be attained. Slow start-up times dictate their primary use as stationary power or co-generation plants.

\subsection{The Operating Principle of Fuel Cells}

As outlined in chapter 1.3, fuel cells directly convert chemical energy contained in a fuel into electricity. Depending on the type of fuel cell and the fuel being used, the actual reaction mechanism may differ from this description which is applicable to proton exchange membrane fuel cells, but the underlying concept remains the same.

Within the PEFC, hydrogen and oxygen are converted into water while generating electricity. A schematic diagram of the processes occurring in 
a PEFC is shown in Figure 1.3. The electrochemical reaction of those processes can be noted as

$$
\mathrm{H}_{2}+\frac{1}{2} \mathrm{O}_{2} \rightarrow \mathrm{H}_{2} \mathrm{O}
$$

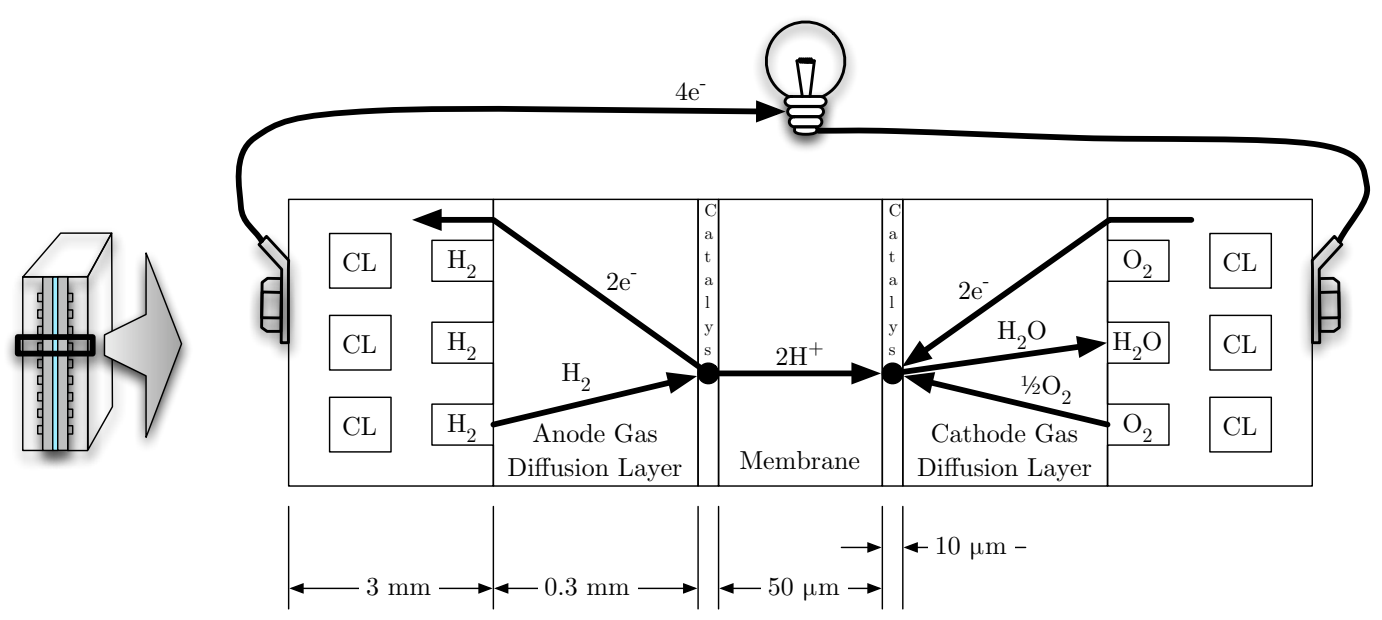

Figure 1.3: Schematic representation of a PEM fuel cell. The drawing is not to scale, but typical dimensions have been indicated on the drawing.

This overall reaction takes place in two, spatially separated, half reactions, shown on the left and right hand side of Fig. 1.3 respectively and given by:

$$
\begin{gathered}
\frac{1}{2} \mathrm{O}_{2}+2 e^{-}+2 \mathrm{H}^{+} \stackrel{\text { catalyst }}{\longrightarrow} \mathrm{H}_{2} \mathrm{O} \\
\mathrm{H}_{2} \stackrel{\text { catalyst }}{\longrightarrow} 2 \mathrm{H}^{+}+2 e^{-}
\end{gathered}
$$

(1.3) is typically termed the Hydrogen Oxidation Reaction (HOR) . (1.2) is composed of two reactions: the Oxygen Reduction Reaction (ORR) (1.4) and the reaction recombining the ionized species into product water (1.5):

$$
\begin{gathered}
\frac{1}{2} \mathrm{O}_{2}+2 e^{-} \stackrel{\text { catalyst }}{\longrightarrow} O^{2-} \\
\mathrm{O}^{2-}+2 \mathrm{H}^{+} \rightarrow \mathrm{H}_{2} \mathrm{O}
\end{gathered}
$$

The ORR and HOR give rise to a voltage difference across the whole cell that describes the transformation of chemical energy into electrical 
energy. If no net current is drawn from the cell, this voltage difference can be determined from thermodynamic properties for a given reaction and conditions (pressure, temperature) - see chapter 2.2 for more details.

In order to technically exploit such a reaction, a fuel cell is composed of an anode compartment containing the fuel (typically gaseous hydrogen) and a cathode compartment filled with the oxidant gas (typically oxygen contained in ambient air). The two chambers are separated through an electrically insulating (i.e. no electron conduction) and gas impermeable membrane - the electrolyte - that is capable of conducting protons. The area in contact with the membrane is covered with a platinum catalyst both on the anode and cathode side and is therefore referred to as a catalyst layer.

Adjacent to the catalyst layer on both sides of the membrane is a porous, electrically conducting gas diffusion layer (GDL). It allows reaction gases (i.e. hydrogen and oxygen) to flow to the reaction sites on the catalyst layer and product water to flow back out.

The gas diffusion layers are held in place by gas impermeable, electrically conducting plates. Gas channels are machined into these so called bipolar plates in order to distribute hydrogen and oxygen evenly over the membrane surface.

From the anode inlet, hydrogen is distributed over the whole cell surface from where it diffuses across the gas diffusion layer to the catalyst layer. Upon contact with the catalyst in the anode compartment, the hydrogen atoms are split into a proton $\left(H^{+}\right)$and an electron $\left(e^{-}\right)$. The electron cannot cross the membrane and leaves the fuel cell as electric current through the gas diffusion layer and the bipolar plate. The proton on the other hand is transported across the membrane to the cathode side.

On the cathode side, oxygen enters the fuel cell, is distributed over the cell surface by the gas channels, diffuses to the catalyst layer and again through the presence of the platinum catalyst - the oxygen atom recombines with the proton and the electron to form water. The product water then diffuses back through the gas diffusion layer and leaves the fuel cell through the gas channels.

The heat generated during the electrochemical reaction is transferred to the bipolar plates through conduction. From there, it is either transmitted to the ambient air through specially designed cooling fins or it is evacuated from the system through a cooling liquid (CL) circulating within the bipolar plates. 
Depending on the current density (i.e. the current per unit of cell surface area), electrical voltages between $1 V$ and $0.5 V^{1}$ are typically achieved. The fuel cell's characteristics and operating conditions determine the maximum current density and power that can be achieved per cell.

In order to increase the power and voltage of a fuel cell, a number $\left(n_{F C}\right)$ of single cells are stacked to form the so-called fuel cell stack. Such a configuration corresponds to an electrical series connection of the single elements. The reaction gases and cooling liquid are supplied to the cells through a parallel network of supply channels.

\subsection{Motivation of the Present Work}

If fuel cell technology is to be used in decentralised power sources or in mobile or transportation applications, the systems have to be able to adapt to fast load changes and varying operating parameters.

In order to achieve such performance, the system's behaviour along with its interactions with the different subsystems, typically between the FC, the FC auxiliaries (i.e. reactant supply and conditioning systems), and the electrical system it is being connected to, have to be understood and appropriately modelled. In the words of Pasaogullari et al [7], this can be summarised as:

It is also found that minimisation of performance limitations such as membrane dry-out and electrode flooding depends not only on material characteristics but also on the optimisation of these operating parameters. i.e. the humidification level and flow rate of reactant streams.

Once they are well understood and described in an appropriate model, this can be used to derive a control strategy for the whole FC system that maximises or optimises performance, efficiency and lifetime. Other benefits reside in the potential observability of variables that cannot be measured directly but represent an important parameter for improving system performance (e.g. membrane water content).

The Laboratoire d'Electronique Industrielle (LEI) has a strong interest in electrical power conversion and storage technologies, particularly in the context of future distributed power generation systems based on renewables.

\footnotetext{
${ }^{1}$ The voltage decreases as the current drawn from the fuel cell increases.
} 
Amongst others, Rivas [8] has recently developed a new DC-AC power conversion strategy for use with fuel cell systems. In order to successfully implement such a converter, the dynamic behaviour of the fuel cell system it is connected to has to be better understood.

While full featured 3D [9], 2D [10] or 1+1D [11] finite element models can describe the complicated phenomena occurring in the FC much more accurately, their numerical nature makes them unsuitable for use in an real-time system controller.

In fuel cell systems, the auxiliaries, also called Balance of Plant (BOP) play a significant role in the total performance of the system. Not much work has been reported in the literature related to the total system model and control by taking the auxiliaries into account.

The goal of the present work is therefore to elaborate a computationally non-intensive (ideally fully analytical) fuel cell system model. The model should retain parameters that have a physical significance so that it can be adapted to a given system. It should correctly describe the interaction between the different subsystems (i.e. the fuel cell stack, the reactant supply system, the thermal management system and the electrical power converter) from a controls point of view. Because of these requirements, only the dominant time constants of the system will be considered (mainly related to the auxiliaries) while a steady state, space averaged 1D model is elaborated for the FC stack.

\subsection{Literature Survey}

Ever since the early nineties, fuel cell modelling has become an important subject of research resulting in many different modelling approaches and models. This section aims to give an overview of the major fuel cell models for PEMFCs followed by a more detailed description of models dealing with interaction between a fuel cell stack and its auxiliaries.

The fuel cell models published by Springer et al. [12] and Bernardi et al. [13] were the first to provide complete, isothermal, one-dimensional, steady state models of a complete fuel cell. Their work was based on previous studies focusing on individual fuel cell components (membrane, diffusion electrodes, etc.). Nguyen et al. [14] and Fuller et al. [15] proposed additional models focusing on water and heat management within a PEMFC. Li et al. $[16,17]$ proposed a series of engineering fuel cell models to investigate the effect of various design and operating parameters. 
These models all focused on the description of the fuel cell stack and formed the basis for many 2D [18, 10] and 3D [19, 9, 20, 21] finite element models that allow to predict local reactant concentration, temperature and current density. In order to reduce the required computing power while still retaining detailed, locally resolved results, Freunberger et al. [11] have recently proposed a $1+1 \mathrm{D}$ fuel cell model. Their approach combines an along-the-channel description with a cross-membrane model giving them computationally non-intensive access to spatially resolved temperature, gas concentration and current density data.

Amphlett et al. [22, 23] developed a parametric fuel cell model that was then fitted to data from a Ballard Mk. IV fuel cell. Kim et al. [24], Lee et al. [25], and Mann et al. [26] proposed generic models that can be adjusted to a given fuel cell by adjusting a certain number of fitting parameters.

All of the above models provide valuable insight into the governing processes within a fuel cell stack. Since they focus on the fuel cell stack only, they cannot be directly used for developing control strategies for a fuel cell system. Additionally, the spatially resolved models are too complex and computationally intensive to be used for real-time applications. The simpler, parametric models do not resolve the system's dependence on operating conditions and therefore cannot provide any information relevant to system control.

Rodatz [27] developed a one dimensional, steady state fuel cell model. Based on stack temperature, current, anode and cathode humidities and pressures, it describes the cell voltage.

The overall cell voltage is determined as an open circuit voltage from which the activation losses (described in a Tafel equation) and ohmic membrane losses (determined from the membrane water content) are subtracted. The model does not take into account the modification of the average gas composition by the water transfer across the cell, and cannot be solved analytically.

This thesis provides an approach to overcome these deficiencies by proposing a model that does take into account the modification of the average gas composition by the water transfer across the cell, and can be solved analytically [28].

Furthermore, Rodatz developed a dynamic model of the air supply system. It describes the dynamics of the air mass flow rates into and out of the stack along with the pressure evolution in the inlet and outlet manifolds. The compressor is modeled based on a compressor map and provides the 
mass flow into the inlet manifold. A proportional valve regulates the airflow out of the exhaust manifold. The resulting dynamic air supply system model was then used to develop a model based controller that showed excellent performance with respect to response time.

Pukrushpan et al. [29] have proposed a fuel cell system model to be used for determining control laws and strategies. The air supply system model is very similar to the one described by Rodatz. It consists of a compressor characterised by its map and the pressure dynamics are the result of a mass balance on the inlet and outlet manifold. The flow rates into and out of the stack are given through the nozzle equation.

Based on the operating parameters described in the air supply model, a steady state stack model determines the fuel cell voltage. The stack model describes the overall voltage as an equilibrium voltage from which activation, concentration and ohmic (ionic) losses are deducted. The equilibrium voltage is based on the Nernst potential, activation overpotential is determined by a Tafel equation, ohmic overpotential based on water content dependent membrane resistance and concentration overpotential is expressed as a function of a limiting current density.

Gas transport across the gas diffusion layer is not explicitly modelled but is contained in the limiting current density notation. The average gas and water concentration in the gas channels is based on the inlet and outlet flow rates that are each given by the nozzle equation. This model is then used to determine multivariable controllers for the cathode oxygen supply. For redundancy in case of sensor failure, real time observers are calculated to estimate stack variables.

Because of the description of the diffusion overpotential in terms of a limiting current density, the model cannot reproduce a change in gas transport properties that is due to different operating conditions.

In collaboration with Pukrushpan et al., Miotti et al. [30] have recently proposed a control oriented model for a PEM fuel cell system where the same compressor map, filling-emptying approach is kept for the description of the air supply subsystem. The fuel cell is modelled (including water transport across the cell) through a map obtained from a numerical $1+1 \mathrm{D}$ model.

Hernandez et al. [31] are currently developing a non-linear, state space model of a PEMFC. It describes stack performance based on the description of the gas pressure evolution in the gas channels along with a probabilistic description of water condensation within the system. The model provides 
an approach to describe water condensation within the fuel cell but requires computationally intensive, iterative solution procedures that make it unsuitable for real-time applications.

\subsection{Contributions}

In order to successfully control and run a fuel cell system, the user (or a controller) can act on various actuators (i.e. air compressor, pressure reduction valve, heat exchanger, etc.).

Ideally, each of those actuators would be used to adjust its associated controllable variable that would then affect only one desired state within the FC system. Unfortunately, by modifying an actuator variable, more than just one state of the system is affected. In order to keep the system in its optimum state, a controller should be capable of adjusting all subsystems such that no such cross coupling phenomena occur.

This thesis provides an analytical, space-averaged PEM fuel cell system model that can be used to devise such a controller.

This is achieved by providing an analytical, steady state model for the processes occurring in the fuel cell. The model resolves the fuel cell performance based on the operating conditions (i.e. reactant pressure, flow rate and humidity along with fuel cell current and temperature). An algebraic solution to the water transport equations across the system addresses the shortcomings of comparable models in literature that require iterative solution procedures.

The fuel cell model's inputs are then linked to the user inputs by describing each of the auxiliary systems in a dynamic, state-space approach. On the air supply system's side, the resulting state space model directly links the voltage applied to a volumetric air compressor to the resulting air flow rate and pressure. This eliminates the need for a compressor map that is typically used to describe the air supply system in literature models.

On the hydrogen supply system's side, the evolution of the hydrogen mass flow is described as a function of the voltage supplied to the recirculation pump. This is an auxiliary system that has so far not been modelled by any control oriented fuel cell system model in literature.

The thermal management system is described by an analytical, dynamic set of equations that describe the temperature evolution in the system as a function of the fuel cell's operating point along with the user's interaction with the cooling circuit. This provides an additional element in a control 
oriented fuel cell system model that has not been addressed in literature so far.

The resulting system model can then be used as a basis for developing advanced fuel cell system controllers or to implement real-time maximum efficiency point tracking algorithms [32].

\subsection{Structure of this Document}

Chapter 1 has introduced the reader to the context of fuel cell technology and then summarised the motivation of the present work along with a literature survey and a presentation of the contributions of this thesis.

Chapter 2 describes how the fuel cell stack is modelled in the context of this thesis. The first section introduces the electrochemical reactions occurring in the cell. Afterwards, the fuel cell performance is described in terms of an equilibrium voltage from which the different losses are deducted. The losses are explained and analytical descriptions are derived for each of them.

In order to link the derived performance equations to the fuel cell's operating conditions, species transport within the fuel cell needs to be described. A mass balance based approach to determine average gas concentrations along the gas channel will be developed. This is then used as a basis for describing reaction gas and water transport in the direction perpendicular to the cell.

While similar approaches have been used to numerically calculate species transport across the cell in iterative schemes, a novel technique for analytically describing species transport in steady state conditions is presented.

The resulting averaged, 1D, steady state fuel cell model that describes cell performance as a function of the operating conditions is summarised in chapter 2.7.

Chapter 3 focuses on the system's auxiliaries responsible for providing reaction gas flow and conditioning along with temperature control. Based on the physical processes in each subsystem, dynamic (state space inspired) descriptions linking the user (or ultimately controller) input to the resulting operating conditions for the fuel cell stack are derived.

Chapter 4 focuses on the design of the test bench used for the experimental verification of the developed models. It describes first the balance of plant components installed to maintain the fuel cell in any desired operating 
condition. A second part outlines the data acquisition and control system that was designed to monitor and control the fuel cell system.

Chapter 5 presents experimental results from the PEMFC test bench installed at LEI. For each model presented in chapters 2 and 3, the model parameters are identified. Subsequent experimental results are then used to validate the models.

In Chapter 6, the general conclusions of the thesis are presented and recommendations are made for future research work on the topic of modelling and control of fuel cells. 

Fuel Cell Stack Model 


\subsection{Introduction}

This chapter presents an analytical PEM fuel cell stack model that describes its performance (i.e. the overall voltage) based on physical system inputs. These inputs - shown in Table 2.1 - can be due to the environment or other constant operating conditions (i.e. ambient pressure), to an interaction with the user (i.e. fuel cell current) or can be provided by the auxiliary systems (i.e. air mass flow).

\begin{tabular}{llr}
\hline Variable Name & Operating Parameter & Unit \\
\hline $\mathbf{I}_{\mathbf{e l}}$ & Fuel cell current & {$[\mathrm{A}]$} \\
$\mathbf{p}_{\mathbf{a i r}}$ & Air inlet pressure & {$[\mathrm{Pa}]$} \\
$\mathbf{p}_{\mathbf{H}_{\mathbf{2}}}$ & Hydrogen inlet pressure & {$[\mathrm{Pa}]$} \\
$\mathbf{p}_{\mathbf{a m b}}$ & Ambient pressure & {$[\mathrm{Pa}]$} \\
$\mathbf{\mathbf { m }}_{\mathbf{a i r}}$ & Air inlet mass flow rate & {$[\mathrm{kg} / \mathrm{s}]$} \\
$\dot{\mathbf{m}}_{\mathbf{H}_{\mathbf{2}}}$ & Hydrogen inlet mass flow rate & {$[\mathrm{kg} / \mathrm{s}]$} \\
$\psi_{\text {air }}$ & Air inlet humidity & {$[-]$} \\
$\psi_{\mathbf{H}_{\mathbf{2}}}$ & Hydrogen inlet humidity & {$[-]$} \\
$\mathbf{T}_{\text {stack }}$ & Stack temperature & {$[\mathrm{K}]$} \\
\hline
\end{tabular}

Table 2.1: FC model inputs and FC operating parameters.

In order to arrive at such a performance description, a first section recalls the performance equations that describe the overall fuel cell voltage as certain equilibrium voltage - termed the Nernst voltage and given by (2.4) - from which losses are deducted ${ }^{1}$.

$$
V_{F C}=E_{0}-\eta_{a c t}-\eta_{c o n c}-\eta_{o h m}-\eta_{i o n}
$$

These losses typically depend on the $\mathrm{O}_{2}$ and $\mathrm{H}_{2}$ concentration at the reaction sites along with the water content of the membrane ${ }^{2}$ which need to be determined.

In order to model the reactant concentration at the catalyst layer, a second part outlines species transport within the fuel cell which ties the

\footnotetext{
${ }^{1}$ The equilibrium voltage and losses are calculated for one cell. The results are then simply multiplied by the number of cells to obtain the overall fuel cell voltage.

${ }^{2}$ The ionic resistance of the membrane decreases with increasing water content, see chapter 2.3.4 for more details.
} 
performance equations to the operating conditions and system inputs. Water transport - detailed in section 2.5 - characterises the evolution of the membrane humidification state while oxygen and hydrogen transport outlined in section 2.4 - determines the respective gas concentrations at the reaction sites.

The basic performance equations along with the underlying description of gas and water transport have been adopted from literature. Where different formulations for the same phenomenology exist, they are compared qualitatively and quantitatively to justify their inclusion in a control system oriented model. Thereon, a novel approach to determine average gas and water concentrations in the gas channel forms the basis for an analytical description of water and gas transport within the system.

\subsection{Fuel Cell Equilibrium Voltage}

Thermodynamics state that the maximum obtainable energy from a chemical reaction corresponds to the Gibbs free enthalpy of reaction $\Delta \mathcal{G}$.

$$
\Delta \mathcal{G} \equiv \Delta \mathcal{H}-T \Delta \mathcal{S}
$$

\begin{tabular}{lcccc}
\hline End product & $\begin{array}{c}\Delta \mathcal{G}_{0} \\
{[\mathrm{~kJ} / \mathrm{mol}]}\end{array}$ & $\begin{array}{c}\Delta \mathcal{H}_{0} \\
{[\mathrm{~kJ} / \mathrm{mol}]}\end{array}$ & $\begin{array}{c}\Delta \mathcal{S}_{0} \\
{[\mathrm{~J} /(\mathrm{mol} \mathrm{K})]}\end{array}$ & $\begin{array}{c}E_{00} \\
{[\mathrm{~V}]}\end{array}$ \\
\hline $\mathrm{H}_{2} \mathrm{O}_{\text {liquid }}$ & -237.2 & -285.9 & -163.2 & 1.23 \\
$\mathrm{H}_{2} \mathrm{O}_{\text {gaseous }}$ & -228.6 & -241.8 & -44.38 & 1.19 \\
\hline
\end{tabular}

Table 2.2: Molar reaction enthalpies and equilibrium voltage $E_{00}$ for the fuel cell reaction $\mathrm{H}_{2}+\frac{1}{2} \mathrm{O}_{2} \rightarrow \mathrm{H}_{2} \mathrm{O}$ for standard reaction conditions (i.e. $T_{0}=25^{\circ} \mathrm{C}$ and $p_{0}=101^{\prime} 325 \mathrm{~Pa}$ ). Data from [33].

Conservation of energy directly relates the molar Gibbs free enthalpy of reaction to the maximum obtainable fuel cell voltage $E_{0}$ :

$$
\Delta \mathcal{G}=-n F E_{0}
$$

Since $\Delta \mathcal{G}$ is dependent on reactant pressure and temperature, the obtainable voltage for a given set of reaction parameters is given as a standard equilibrium voltage $E_{00}$ corrected by the deviation from those standard 
conditions $^{3}$. This results in the Nernst equation (2.4) that gives the equilibrium voltage $E_{0}$ for a given reaction as a function of temperature and reactant pressures if no current is drawn from the fuel cell. As soon as a net current is produced by the fuel cell, equilibrium conditions no longer apply and voltage losses occur in the cell.

$$
\begin{aligned}
& E_{0}=E_{00}+\frac{\Delta \mathcal{S}_{0}}{n F}\left(T-T_{0}\right)+\frac{\mathcal{R} T}{n F} \ln \left(\frac{\prod_{i}\left(\frac{p_{i}}{p_{0}}\right)^{\gamma_{i}}}{\prod_{j}\left(\frac{p_{j}}{p_{0}}\right)^{\gamma_{j}}}\right) \\
& \text { where: } E_{00}=\text { equilibrium potential at stand- }[V] \\
& \text { ard conditions (i.e. } p_{i}=p_{0}= \\
& 101^{\prime} 325 P a \text { and } T=298 K \text { ) } \\
& p_{i}=\text { partial pressure of educt } \mathrm{i} \quad[\mathrm{Pa}] \\
& p_{j}=\text { partial pressure of product } \mathrm{j}^{4} \quad[\mathrm{~Pa}]
\end{aligned}
$$

\subsubsection{Mixed Potential}

As shown above, the equilibrium voltage at standard conditions $E_{00}$ for the $\mathrm{H}_{2}+\frac{1}{2} \mathrm{O}_{2} \rightarrow \mathrm{H}_{2} \mathrm{O}$ reaction is roughly $1.23 \mathrm{~V}$. Unfortunately, in the case of PEFCs, water formation from hydrogen and oxygen is not the only reaction occurring in the cell.

Amongst others, hydrogenperoxide is formed at the cathode according to:

$$
\mathrm{H}_{2}+\mathrm{O}_{2} \rightarrow \mathrm{H}_{2} \mathrm{O}_{2}
$$

This undesirable side reaction is characterised by an equilibrium potential $E_{00}=0.682 \mathrm{~V}$. This along with other (less significant side reactions) leads to an observable overall equilibrium voltage of

$$
E_{0, n e t} \approx 1 \mathrm{~V}
$$

\footnotetext{
${ }^{3}$ The derivation of the Nernst equation can be found in Appendix A.1.

${ }^{4}$ For clarity and in order to free the reader from having to remember the sign change of $\gamma_{i}$ for educts and products, products are directly listed in the denominator of the logarithm.
} 


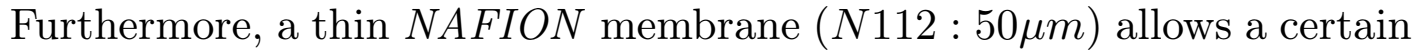
amount of $\mathrm{H}_{2}$ crossover further reducing the open circuit potential. Typical values for the $B R E S A^{5}$ stack are in the order of $950-970 \mathrm{mV}$.

\subsection{Fuel Cell Losses}

As mentioned above, the Nernst equation (2.4) gives the equilibrium voltage $E_{0}$ for a given reaction as a function of temperature and reactant pressures if no current is drawn from the fuel cell. As soon as a net current is produced by the fuel cell, equilibrium conditions no longer apply and voltage losses occur in the cell. Those losses are associated with different physical phenomena and it is typically distinguished between:

Activation losses $\left(\eta_{\text {act }}\right)$ that describe the resistance to charge transfer at each of the half cells. They are the principal source of losses at low current densities and are described more in detail in chapter 2.3.1.

Concentration losses $\left(\eta_{\text {conc }}\right)$ that describe the losses due to gas transport losses in the cell. Since gas transport in the cell is mainly by diffusion, these losses are also termed diffusion losses (or overpotentials). They are responsible for the steep drop-off of cell voltage at high current densities and are explained in chapter 2.3.2.

Electronic conduction losses $\left(\eta_{o h m}\right)$ that describe the losses due to electron conduction in the catalyst layer, the gas diffusion layer and the bipolar plates. They are directly proportional to current density and partly characterise the linear region in the I-V curve. More details can be found in chapter 2.3.3.

Ionic losses $\left(\eta_{i o n}\right)$ that describe the losses due to proton transport through the polymer membrane. Like the electronic conduction losses, they can be described as an ohmic overpotential. However, in the case of the ionic overpotential, the membrane resistance to proton transport is strongly dependent on the membrane water content. Chapter 2.3.4 shows these characteristics in more detail.

${ }^{5}$ BRESA is the PSI and ETHZ internal denomination for the fuel cell stack considered here [34]. 
Note that in the context of this thesis, the above losses have been considered independent of each other. This is correct for the gas diffusion layer and the membrane region but represents an approximation within the catalyst layer where those processes are highly interlinked.

\subsubsection{Activation Overpotential}

An activation overpotential is associated with activation energy required to initiate a charge transfer. The losses (overpotential) will be larger the higher the rate of charge transfer (i.e. the higher the current drawn from the fuel cell).

This applies to any charge transfer occurring in a Redox reaction. In the case of fuel cells, this corresponds to the oxygen reduction reaction at the cathode (1.4) and the hydrogen oxidation reaction (1.3).

The activation energy required for a charge transfer in an electric field can be expressed for the forward and backward reaction occurring at either electrode. This results in the Butler-Volmer ${ }^{6}(\mathrm{BV})$ equation for a redox overpotential, relating net current density in a redox reaction to the corresponding overpotential at a given electrode as a function of the exchange current density $I_{0}^{\prime \prime}$.

$$
I^{\prime \prime}=I_{0}^{\prime \prime}\left[\exp \left(-\frac{\beta n F \eta_{a c t}}{\mathcal{R} T}\right)-\exp \left(+\frac{(1-\beta) n F \eta_{a c t}}{\mathcal{R} T}\right)\right]
$$

For large overpotential values, one of the exponential terms in 2.7 tends to zero. Neglecting the term approaching zero yields the Tafel equation for the cathode and anode respectively.

$$
\begin{gathered}
I_{c}^{\prime \prime}=I_{0, c}^{\prime \prime} \exp \left(-\frac{\beta n F \eta_{a c t, c}}{\mathcal{R} T}\right) \\
I_{a}^{\prime \prime}=-I_{0, a}^{\prime \prime} \exp \left(+\frac{(1-\beta) n F \eta_{a c t, a}}{\mathcal{R} T}\right)
\end{gathered}
$$

The Tafel equation is a simplified description of the current density vs activation overpotential characteristics. It is a very good approximation of the real behaviour at higher current densities. At lower current densities,

\footnotetext{
${ }^{6}$ More details on the derivation of the Butler-Volmer equation can be found in Appendix A.2.
} 
the activation overpotential is described less precisely. It can be shown that while the current density is above roughly 3 times the exchange current density, the error in overpotential is below $10 \%$. For system characteristics typically encountered in fuel cell applications, this means that above roughly $5 \%$ stack load the Tafel equation correctly describes the activation overpotential.

Note that the underlying assumption of describing the activation overpotential through the Butler-Volmer or Tafel equation consists in considering the redox-reaction as an interface reaction. This neglects the space dependence and interlinkage of the reaction with ion, electron and gas transport in a physical three phase boundary (catalyst) layer. For a well designed, well humidified catalyst layer, this assumption is usually verified.

For typical PEFC applications, it can be shown that the anode exchange current density is considerably larger than the cathode one ${ }^{7}$ (see Tab. 2.3). This means that the activation losses (overpotential) at the anode are very small compared to the ones on the cathode and can generally be neglected [13].

\begin{tabular}{ccc}
\hline & Anode & Cathode \\
\hline$I_{0}^{\prime \prime}\left[A / \mathrm{cm}^{2}\right]$ & $1-2$ & 0.01 \\
\hline
\end{tabular}

Table 2.3: Typical exchange current densities for PEFCs (Data from [35]).

The overall activation overpotential can therefore be expressed as:

$$
\eta_{a c t, c}=\frac{\mathcal{R} T}{\beta n F}\left[\ln \left(I_{0, c}^{\prime \prime}\right)-\ln \left(I_{c}^{\prime \prime}\right)\right]
$$

The reaction symmetry coefficient $\beta$ is generally assumed to be $0.5^{8}$. The exchange current density $I_{0, c}^{\prime \prime}$ is a function of the oxygen concentration at the reaction interface along with the temperature. This dependence has

\footnotetext{
${ }^{7}$ The activation energy required to initiate a charge transfer for the ORR is substantially larger than the one required for the HOR.

${ }^{8}$ Other sources [36] have suggested a temperature dependence of $\beta$ for the ORR but for reasons of simplicity a constant $\beta=0.5$ is assumed for the following developments.
} 
been explained by Amphlett et al. [23]. Its expression can be substituted into (2.10) to yield ${ }^{9}$ :

$$
\eta_{a c t}=\frac{\mathcal{R} T}{\beta n F}\left[-\frac{\Delta \mathcal{G}_{a c t}}{\mathcal{R} T}+\zeta+(1-\beta) \ln \left(c_{O_{2}, S}\right)-\ln \left(I_{c}^{\prime \prime}\right)\right]
$$

$\zeta$ is a function of different parameters characterising the reaction sites ${ }^{10}$ it is used as a curve fitting parameter in the context of this model.

\subsubsection{Diffusion Overpotential}

The Nernst equation (2.4) expresses the equilibrium voltage for a set of operating conditions (temperature, reactant pressures). If no current is drawn, the reactant concentrations correspond to those in the gas channel. As soon as a certain net current is drawn from the fuel cell, reactants have to diffuse to the catalyst layer at a rate corresponding to the electric current (see chapter 2.4.1). This diffusion reduces the reactant concentration at the reaction surface.

In order to express the overpotential associated with gas transport, a Nernst potential for partial reactant pressures in the gas channel $E_{0, G C}$ and a Nernst potential corresponding to the partial reactant pressures at the reaction surface $E_{0, S}$ is considered.

The diffusion overpotential $\eta_{\text {conc }}$ will be defined as the difference between the two and can be written as:

$$
\eta_{\text {conc }}=E_{0, G C}-E_{0, S}=-\frac{\mathcal{R} T}{n F} \ln \left(\frac{\prod_{i} p_{i, G C}^{\gamma_{i}}}{\prod_{j} p_{j, G C}^{\gamma_{j}}} \frac{\prod_{j} p_{j, S}^{\gamma_{j}}}{\prod_{i} p_{i, S}^{\gamma_{i}}}\right)
$$

This approach presents the benefit of giving an expression for the diffusion overpotential. However, since the goal is to determine the overall cell voltage for a given set of operating parameters $E_{c e l l}=E_{0, G C}-\eta_{d}-\eta_{\text {others }}$, it is easier to directly calculate the cell voltage for the partial reactant pressures at the reaction surface:

$$
E_{0, S}=E_{0, G C}-\eta_{c o n c}=E_{00}+\frac{\Delta \mathcal{S}}{n F}\left(T-T_{0}\right)-\frac{\mathcal{R} T}{n F} \ln \left(\frac{\prod_{i} p_{i, S}^{\gamma_{i}}}{\prod_{j} p_{j, S}^{\gamma_{j}}}\right)
$$

\footnotetext{
${ }^{9}$ Since the cathode activation overpotential is the only activation loss considered, it is directly noted with the suffix "act" instead of "act,c".

${ }^{10}$ See [23] for more details.
} 
Some literature models describe the diffusion overpotential as a function of the cell's limiting current density ${ }^{11}$. However, since the limiting current density is not a constant for a given cell, but depends on the reactant concentration in the gas channel, such a description is only accurate for a given set of operating parameters and is therefore less useful for modelling a cell under varying operating conditions.

\subsubsection{Electronic Ohmic Overpotential}

The ohmic overpotential within a fuel cell is due to electron conduction in the gas diffusion layer, the catalyst layer, the bipolar plates and the current collector plates. Typically, the ohmic resistance of the current collector plates and the losses in the catalyst layer can be neglected with respect to the other contributions ${ }^{12}$.

Assuming that the electrical resistance in the bipolar plates and the diffusion layer can be determined, the overall ohmic overpotential is given as:

$$
\eta_{o h m}=\left(R_{d i f}+R_{b i p}\right) I_{e l}=R_{e q} I_{e l}
$$

A first estimation of the respective ohmic resistances can be obtained from extrapolation starting from the bulk conductivities. Effective crosssectional areas and conduction lengths have to be defined. Marr et al. [16] have proposed a methodology to obtain the overall electronic bipolar plate resistance using the gas channel geometry along with the bulk material conductivity.

A similar approach has been proposed for the gas diffusion layer. However, unclear contact resistance, unknown void fractions (especially when compressed within the cell) make this approach difficult to exploit. For a given stack it is easier to measure the overall equivalent resistance $R_{e q}$ directly.

\footnotetext{
${ }^{11}$ See Appendix A.3 for more information on that approach.

${ }^{12}$ Certain references [37], [16] have proposed a one dimensional description of the catalyst layer taking into account overpotential variation due to electronic resistance. Results show however, that the contribution due to the electron transport resistance is negligible with respect to the activation overpotential.
} 


\subsubsection{Ionic Overpotential}

The ionic overpotential is due to the resistance in proton transport within the polymer electrolyte membrane. As will be seen later on (see chapters 2.4 and 2.5), the membrane region not only acts as a transport media to protons but also to water molecules. Both transport phenomena are closely coupled.

This chapter will give a brief introduction to polymer electrolyte membranes. Since the transport properties of the membrane phase - for both water and proton transport - depend strongly on the membrane water content, a means of describing the actual membrane water content is introduced. Different approaches to determine the membrane water content and conductivity are shown and compared.

\section{Membrane Description}

The polymer membranes separating the anode and cathode compartments while conducting protons, exist in a number of different configurations and are available from different vendors. Their working principle is very similar and therefore, only the most widely used material, Nafion by DuPont, will be described here.

Nafion is a modified polyetrafluorethylene (PTFE) or Teflon where certain fluorine molecules in the main chain have been substituted with side chains (see Figure 2.1). These sidechains carry $\mathrm{SO}_{3}^{-}$ions at their tips. These sulfonic acid $\mathrm{SO}_{3}^{-}$groups - as opposed to the main PTFE moleculeare strongly hydrophilic. When hydrated, the protons that naturally attach to the sulfonic acid side chains become mobile and can travel throughout the electrolyte - the higher the water content, the more mobile the protons and therefore the higher the protonic conductivity of the material.

Such polymer membranes are typically characterise by their equivalent weight. It indicates the dry polymer weight per mol of $\mathrm{SO}_{3}^{-}$group. Typical values for Nafion are around 1'100 $\mathrm{g} / \mathrm{mol}$. Additionally, the membranes can be obtained in various thicknesses $-50 \mu \mathrm{m}$ is considered a good compromise between low ionic resistance, mechanical strength and hydrogen crossover.

\section{Membrane Water Content}

Water content within the membrane $\xi$ varies depending on the actual operating conditions. $\xi$ (also denoted $\lambda$ in literature) denotes the number 


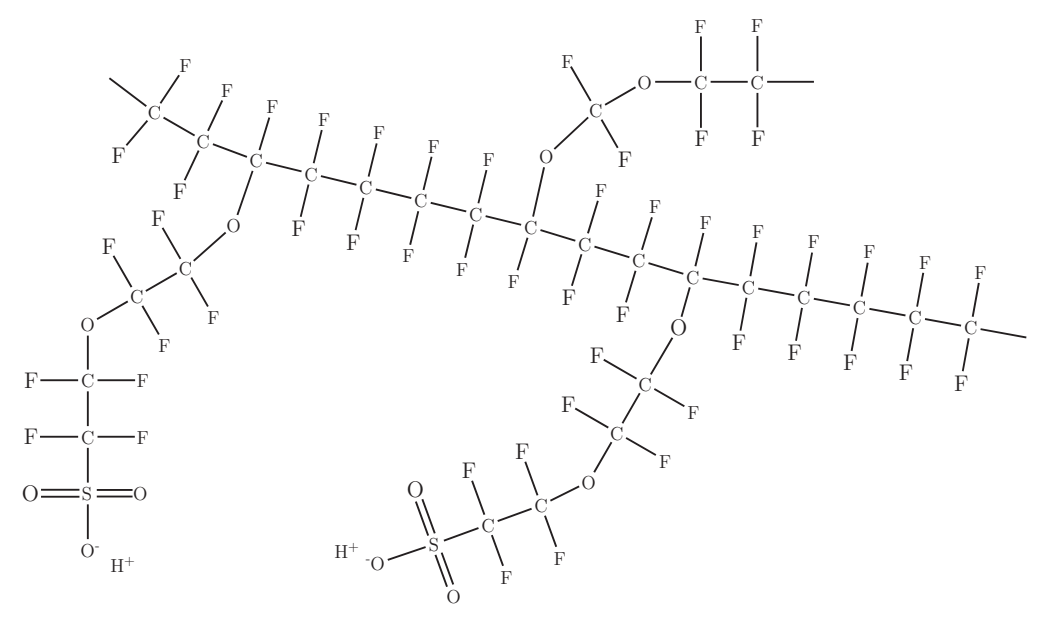

Figure 2.1: Chemical structure of Nafion. The long main chain is the base PTFE material upon which the carrying $\mathrm{SO}_{3}^{-}$side chains have been grafted.

of water molecules in the membrane with respect to the number of charge carrying $\mathrm{SO}_{3}^{-}$sites [12].

$$
\xi=\frac{n_{\mathrm{H}_{2} \mathrm{O}}}{n_{\mathrm{SO}_{3}^{-}}}
$$

For Nafion, the water content $\xi$ varies between 0 and 14 depending on the humidity of the surroundings. Interestingly, when the membrane is in contact with liquid water, the water content $\xi$ can go up to 22 . This discontinuity is generally known as Schroeder's paradox [38].

Water content of a substance in contact with a humid atmosphere is typically a function of the water activity $a$ at the interface. For equilibrium conditions, such a dependence has been curve fitted ${ }^{13}$ for Nafion 117 by Springer et al. [12] for dry gases (i.e. $p_{\mathrm{H}_{2} \mathrm{O}}<p_{\mathrm{H}_{2} \mathrm{O}}^{\mathrm{sat}}$ ):

$$
\xi_{303}=0.043+17.81 a-39.85 a^{2}+36.0 a^{3}, \text { for } 0<a \leq 1
$$

\footnotetext{
${ }^{13}$ The subscript 303 refers to the temperature in $K$ at which the experimental data was recorded.
} 
For humid gas mixtures up to $\chi_{\mathrm{H}_{2} \mathrm{O}}=3 \chi_{\mathrm{H}_{2} \mathrm{O}}^{\text {sat }}$ the following linear approximation has been used:

$$
\xi_{303}=14+1.4(a-1), \text { for } 1<a \leq 3
$$

Measurements were carried out at $30{ }^{\circ} \mathrm{C}$ but the authors estimate that they are also representative of other temperatures.

Hinatsu et al. [39] have measured the water uptake isotherm at $80^{\circ} \mathrm{C}$ and fitted it to the following equation:

$$
\xi=0.3+10.8 a-16.0 a^{2}+14.1 a^{3}
$$

(2.16), (2.17) and (2.18) have been plotted in Fig. 2.2. They represent membrane water content for a membrane that is in equilibrium (static) with water vapour at a given activity $a$. During operation, such steady state equilibrium conditions are rarely achieved and the membrane water content depends on water transport to, from and within the membrane.

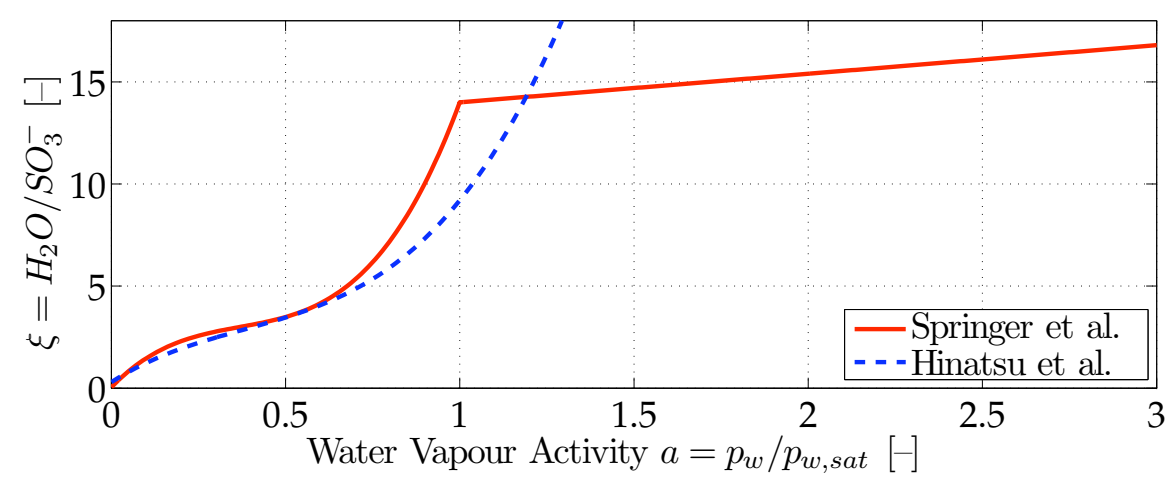

Figure 2.2: Membrane water content as a function of water activity for Nafion 117.

\section{Membrane Conductivity}

It has been widely shown in literature that the membrane conductivity $\sigma_{m}$ is strongly water content dependent [40], [41], [23] and [42]. Theoretical derivations of the overall membrane conductivity (see Appendix A.4) do not exhibit this characteristic since no such dependencies have been reported in literature for the ionic membrane conductivity or the electrokinetic 
permeability - parameters that theoretically characterize ionic membrane resistance.

Therefore, experimental data from literature was adopted to describe the variation of overall membrane resistivity with water content.

Springer et al. [12] have approximated experimental data to fit the following relationship ${ }^{14}$ :

$$
\sigma_{m}^{0}=0.5139 \cdot \xi-0.326, \text { for } \xi>1
$$

The resulting water-content-dependent membrane conductivity $\sigma_{m}^{0}$ at $30^{\circ} \mathrm{C}$ can then be adjusted for temperature dependence according to:

$$
\sigma_{m}=\sigma_{m}^{0} \exp \left[1268\left(\frac{1}{303}-\frac{1}{T}\right)\right]
$$

Mann et al. [26] have used data from Büchi and Scherer [40] to establish a correlation for the overall Nafion membrane resistivity $r_{m}$. It is expressed as (note that in (2.21), current density is expressed in units of $\mathrm{A} / \mathrm{cm}^{2}$ ):

$$
r_{m}=\frac{1.816\left[1+0.03 I^{\prime \prime}+0.062\left(\frac{T}{303}\right)^{2} I^{\prime \prime 2.5}\right]}{\left(\xi-0.634-3 I^{\prime \prime}\right) \exp \left[4.18\left(\frac{T-303}{T}\right)\right]}
$$

The exponential factor in (2.21) is the correction factor if the cell is not at $30^{\circ} \mathrm{C}$. The pre-exponential term is a fit of resistance data from [40] corrected for water content $\xi$, current density $I^{\prime \prime}$ and temperature $T^{15}$.

(2.20) and (2.21) have been plotted against membrane water content and are shown in Figure 2.3. Note the almost linear relation between water content and membrane conductivity. Both equations yield qualitatively the same behaviour. An increase in temperature increases membrane conductivity whereas an increase in current density (for (2.21)) has relatively few influence.

For comparison reasons (A.33) has been evaluated for typical literature values (see Table A.1). The resulting conductivities are also shown on

\footnotetext{
${ }^{14}$ Their data was established for Nafion 117. Since Nafion 112 differs from Nafion 117 in thickness only, their data is assumed to correctly describe the water uptake mechanisms in Nafion 112.

${ }^{15}$ Note that the equations have been established for Nafion 117. Even though Nafion 112 has the same structure as Nafion 117, [40] indicate that the increase of membrane resistivity with current density is less pronounced for thinner membranes.
} 


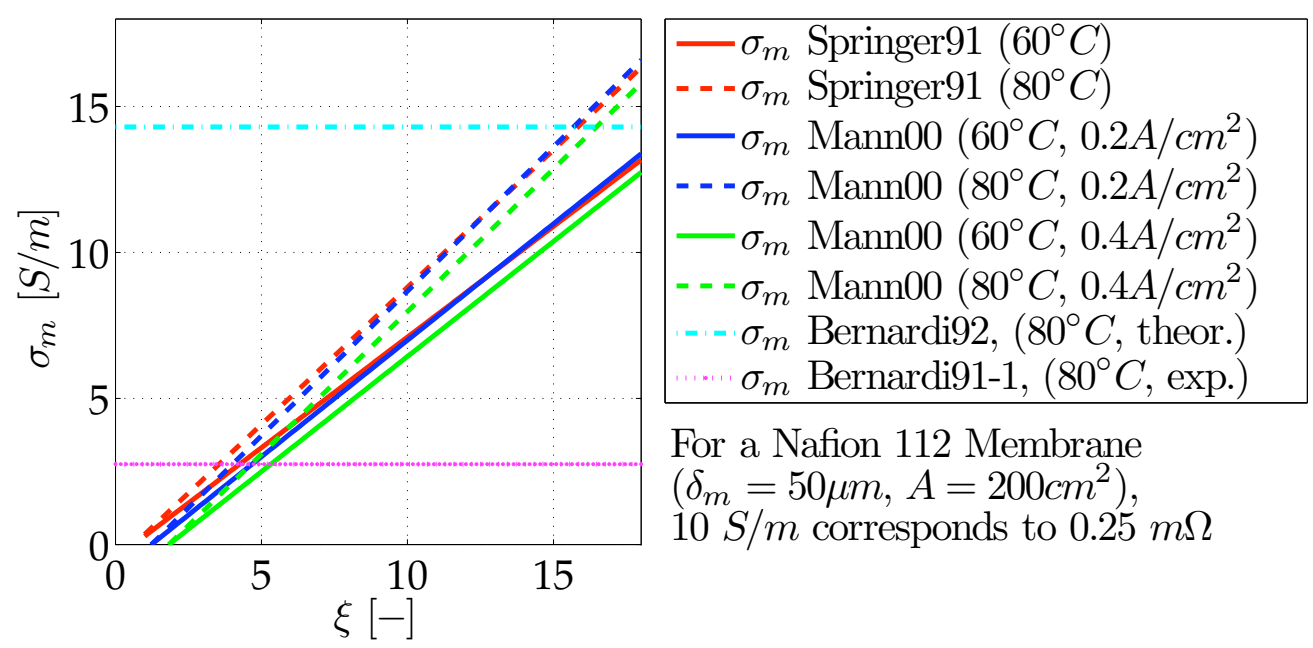

Figure 2.3: Membrane conductivity dependence on membrane water content based on Springer et al. (2.20), on Mann et al. (2.21) and on a theoretical Schlögl/Nernst approach (A.33) (parameter values for (A.33) are taken from Bernardi et al. (see Table A.1)).

Figure 2.3. Since membrane water content was not indicated for values in Table A.1, the values have been plotted as horizontal lines.

Accounting for the small differences between (2.20) and (2.21), the description of the membrane conductivity as in (2.19) and (2.20) has been retained for describing the ionic overpotential. In the context of the model, the base membrane conductivity is expressed as

$$
\sigma_{m}^{0}=k_{\sigma} \cdot \xi-0.326, \text { for } \xi>1
$$

while the temperature dependence of the actual membrane conductivity - expressed in (2.20) - remains unchanged. By fitting the parameter $k_{\sigma}$ to the considered fuel cell, the model can be adjusted for membranes that exhibit different properties than the ones identified in [12].

The ionic overpotential is then given by:

$$
\eta_{i o n}=\frac{\delta_{m} I^{\prime \prime}}{\sigma_{m}}
$$




\subsection{Gas Transport}

In the expression of the overall cell voltage (2.1), both the activation overpotential $\eta_{a c t}$ and the concentration overpotential $\eta_{\text {conc }}$ depend on the reactant concentration at the catalyst layer. Therefore, that concentration needs to be described as a function of the cell's operating condition.

A detailed, space-resolved description of the highly interlinked multiphase species transport (i.e. water and reaction gases) within the fuel cell is typically achieved with different $(3 \mathrm{D}, 2 \mathrm{D}+1 \mathrm{D}, 2 \mathrm{D}$ or $1 \mathrm{D}+1 \mathrm{D})$ finite element analysis models at the expense of simulation time.

In order to obtain a computationally non-intensive, ideally analytical model, while retaining physical significance, a space averaged, 1D description of the occurring processes has been developed in the context of this thesis.

In a first step, section 2.4.2 shows how mass balance considerations on the two gas channels - addressing water and gas transport at the same time - can be employed to yield average species concentrations therein.

Based on these averaged species concentrations, section 2.4.3 then describes gas transport across the GDL based on a simple 1D diffusion process where the diffusion coefficients take the average species composition into account.

Similarly, water transport (section 2.5) across the GDL-membrane-GDL sandwich can then also be analysed in a 1D reference, based on the average concentrations in the gas channel.

Note that this approach is subject to a "chicken and egg" problem since the water flux across the GDL-membrane-GDL sandwich has to be known to determine the average species concentration in the gas channel, which in turn - needs to be established to calculate the water flux perpendicular to the membrane.

This has traditionally been solved through numerical, iterative models. An approach for analytically determining the water transfer within the cell based on the operating conditions will be presented in section 2.5.6.

Figure 2.4 gives a good overview of the occurring gas and water transport processes for the anode and cathode compartments. Reaction gas transport (i.e. oxygen on the cathode side and hydrogen on the anode side) can be attributed to two distinct regions in the fuel cell (gas channel, gas diffusion layer (GDL)), occurring in two spacial directions with different associated transport mechanisms. 


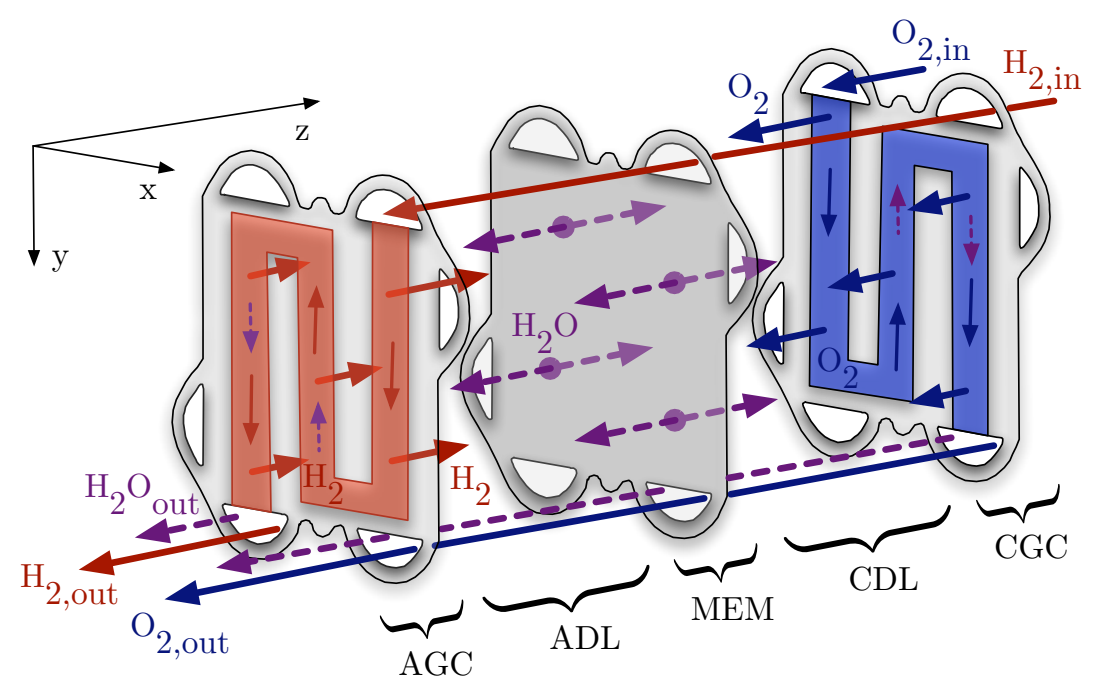

Figure 2.4: Schematic representation of species transport within a PEM fuel cell repeat element. Nitrogen flow is not represented on the cathode side since it does not participate in the electrochemical reactions.

Convective gas transport within the gas channel in the $x$-y-plane is driven by a pressure gradient imposed by the fuel cell auxiliaries (i.e. air compressor or hydrogen pump). However, for the electrochemical reaction to take place in the catalyst layer, a gas flux perpendicular ( $z$-direction) to the main gas flow in the gas channel has to be established. This gas flux is typically due to concentration difference-driven diffusion mechanisms. The molar flow rate of the oxygen flow in the $z$-direction is directly related to the electrical fuel cell current as given in (2.24). Since diffusional transport is concentration related, there is a strong dependency on water transport which directly influences the remaining species concentration.

For the developments in this chapter, only a single cell is considered. The co-ordinate system's $z$-axis originates at the interface between the anode diffusion layer and the membrane and is defined positive in the direction towards the cathode compartment.

\subsubsection{Description of Reactant Supply}

This section outlines an expression for the rate of reactant consumption for a given fuel cell current and introduces a non-dimensional factor $\lambda$ that relates the actual reactant supply to the fuel cell current. 


\section{Faraday's Law}

The basic fuel cell reactions (1.2) and (1.3) can be related to the resulting fuel cell current through Faraday's Law :

$$
\dot{N}_{i}=\frac{I_{e l}}{n_{i} F}
$$

where $n_{i}$ represents the number of moles of exchanged electrons per mol of reactant $i$. Examining (1.2) and (1.3) yields:

$$
\begin{gathered}
n_{\mathrm{O}_{2}}=4 \\
n_{H_{2}}=2 \\
n_{H_{2} \mathrm{O}}=2
\end{gathered}
$$

(2.24) represents the minimum rate of reactant supply that is required to produce the corresponding electrical current.

\section{Stoichiometric Flow Rates}

In order to easily express the actual rate of reactant supply, a non-dimensional factor $\lambda$, termed the stoichiometric flow rate, is introduced. It is defined for the anode and cathode compartments respectively and relates the reactant supply to the current delivered by the fuel cell.

$$
\lambda=\frac{\text { actual reactant supply }}{\text { required reactant supply }}
$$

Accounting for (2.24), this can be written as:

$$
\lambda_{i}=\frac{\dot{N}_{i, \text { actual }} n_{i} F}{I_{\text {el }}}=\frac{\dot{m}_{i} n_{i} F}{\mathcal{M}_{i} I_{\text {el }}}
$$

By taking the air composition into account, $\lambda_{\mathrm{O}_{2}}$ can be converted into a stoichiometric air flow rate $\lambda_{\text {air }}$.

\subsubsection{Gas Channel Concentration Averaging}

The different species flow rates into and out of the anode and cathode gas channels are shown in Figure 2.5 and 2.6. 

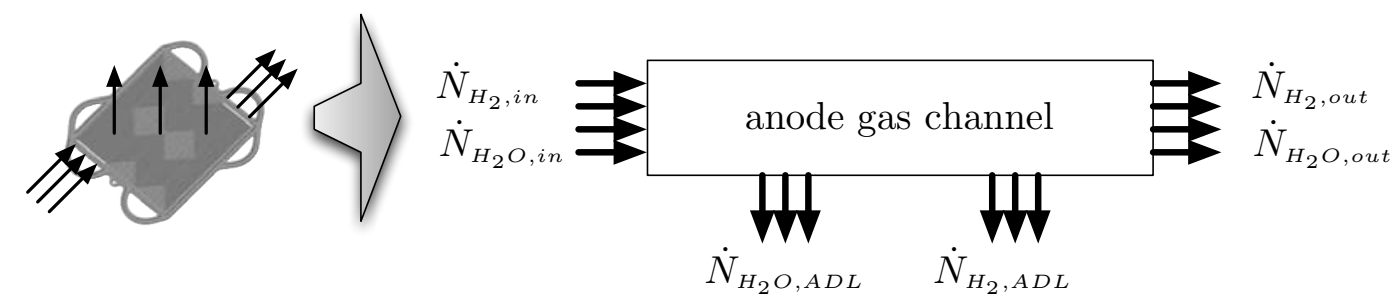

Figure 2.5: Molar inlet and outlet flow rates for the anode gas channel.
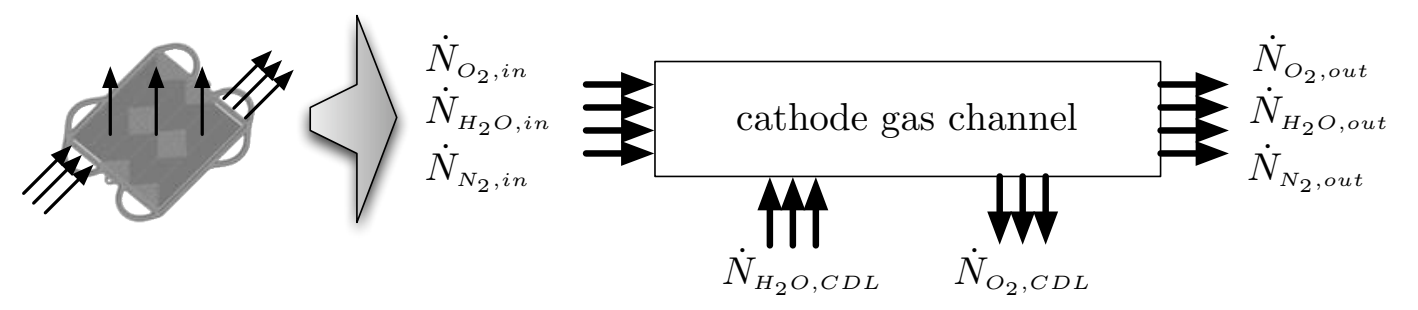

Figure 2.6: Molar inlet and outlet flow rates for the cathode gas channel.

The water flow rates across the anode and cathode GDL are considered to be known for establishing the mass balance (section 2.4.2) and species transport (sections 2.4.3 and 2.5) equations. Once the respective equations are elaborated, they can then be linked to find the overall water flow rates.

\section{Inlet Gas Composition}

As stated in Appendix B.1, dry air is assumed to be composed of $80 \%$ Nitrogen and 20\% Oxygen. For a measured dew point at the fuel cell inlet, the corresponding partial water vapour pressure $p_{v}^{i n}$ can then be determined. For a given overall pressure $p_{L}$, this yields for the inlet cathode gas composition:

$$
\begin{gathered}
\chi_{H_{2} O, c}^{i n}=\frac{p_{v}^{i n}}{p_{L}} \\
\chi_{O_{2}, c}^{i n}=0.2\left(1-\chi_{H_{2} O, c}^{i n}\right) \\
\chi_{N_{2}, c}^{i n}=0.8\left(1-\chi_{H_{2} O, c}^{i n}\right)
\end{gathered}
$$

For the anode compartment a similar approach gives: 


$$
\begin{gathered}
\chi_{H_{2} O, a}^{i n}=\frac{p_{v}^{i n}}{p_{G}} \\
\chi_{H_{2}, a}^{i n}=1-\chi_{H_{2} O, a}^{i n}
\end{gathered}
$$

The molar water fraction for a gas mixture has been plotted as a function of relative humidity, overall gas pressure and temperature in Figure 2.7. Note that at low temperatures and relative humidities, it represents a small portion of the overall gas. However, at high relative humidities or temperatures, it cannot be neglected anymore.

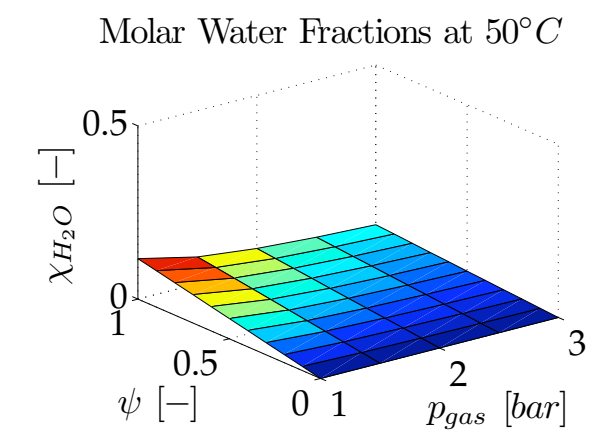

Molar Water Fractions at $70^{\circ} \mathrm{C}$

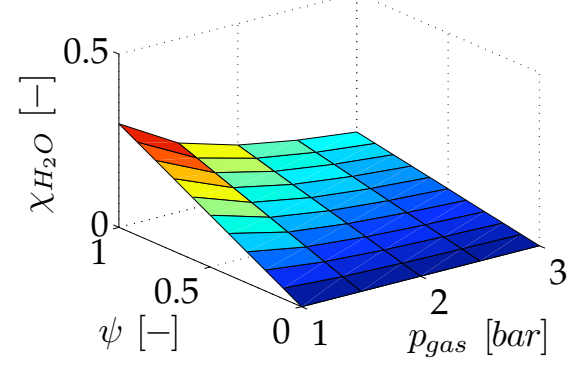

Molar Water Fractions at $60^{\circ} \mathrm{C}$

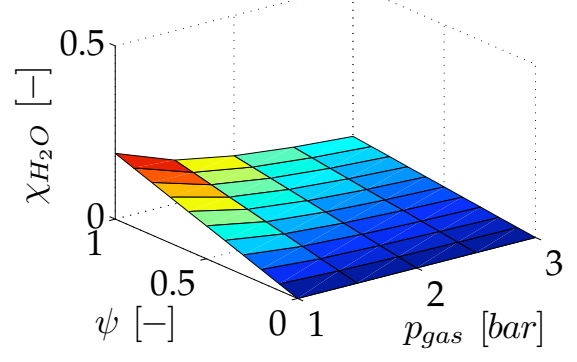

Molar Water Fractions at $80^{\circ} \mathrm{C}$

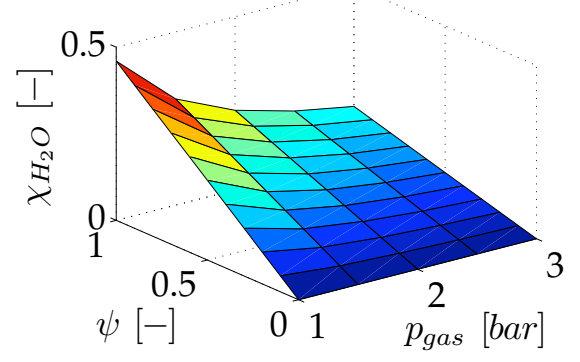

Figure 2.7: Molar water fraction in a gas mixture as a function of relative humidity $\psi$, gas pressure and temperature. See comments on page 35 for the motivation behind running fuel cells at higher gas pressures.

Figure 2.7 also gives an explanation as to why running a fuel cell at higher gas pressures is beneficial for performance. Partial reactant pressure (oxygen or hydrogen) $p_{i}=\chi_{i} p_{G}$ not only increases due to the increased overall gas pressure but also because the water molar fraction decreases (at constant gas dew point or relative humidity). 
The molar flow rates corresponding to the above molar fractions can then be determined using (2.35).

$$
\chi_{i}=\frac{\dot{N}_{i}}{\sum_{i} \dot{N}_{i}}=\frac{\dot{N}_{i}}{\dot{N}_{G}}=\dot{N}_{i} \frac{\mathcal{M}_{G}}{\dot{m}_{G}}=\dot{N}_{i} \frac{\sum_{i} \chi_{i} \mathcal{M}_{i}}{\dot{m}_{G}}
$$

The reactant fluxes in the GDL $\left(\dot{N}_{\mathrm{O}_{2}, C D L}\right.$ and $\dot{N}_{H_{2}, A D L}$ in Figure 2.5 and 2.6) are expressed as a function of the current density according to (2.24). The inlet flow rates for oxygen, nitrogen and hydrogen can therefore be expressed as a function of the stoichiometric flow rate $\lambda$. This yields for the anode and cathode compartments:

$$
\begin{aligned}
& \dot{N}_{O_{2}, c}^{i n}=\lambda_{O_{2}} A \frac{I^{\prime \prime}}{4 F} \\
& \dot{N}_{N_{2}, c}^{i n}=4 \lambda_{O_{2}} A \frac{I^{\prime \prime}}{4 F} \\
& \dot{N}_{H_{2}, a}^{i n}=\lambda_{H_{2}} A \frac{I^{\prime \prime}}{2 F}
\end{aligned}
$$

(2.30) to (2.38) can then be rearranged to result in an expression for the cathode and anode inlet water flow rate.

$$
\begin{aligned}
& \dot{N}_{H_{2} O, c}^{i n}=\frac{\chi_{H_{2} O, c}^{i n}}{1-\chi_{H_{2} O, c}^{i n}} 5 \lambda_{O_{2}} A \frac{I^{\prime \prime}}{4 F} \\
& \dot{N}_{H_{2} O, a}^{i n}=\frac{\chi_{H_{2} O, a}^{i n}}{1-\chi_{H_{2} O, a}^{i n}} \lambda_{H_{2}} A \frac{I^{\prime \prime}}{2 F}
\end{aligned}
$$

\section{Outlet Gas Composition}

Assuming no gas storage in the gas channel, mass conservation dictates that inlet and outlet mass flow be equal.

$$
\dot{m}^{\text {in }}=\dot{m}^{\text {out }}
$$

Air being a mixture of oxygen, nitrogen and water vapour, the above equation can be rewritten in terms of molar flow rates for each component (conservation of species) as shown in Figure 2.6. $\dot{N}_{H_{2} O, C D L}$ denotes the part of the reaction water that diffuses back into the gas channel. 


$$
\begin{gathered}
\dot{N}_{O_{2}, c}^{i n}-\dot{N}_{O_{2}, C D L}=\dot{N}_{O_{2}, c}^{o u t} \\
\dot{N}_{N_{2}, c}^{\text {in }}=\dot{N}_{N_{2}, c}^{\text {out }} \\
\dot{N}_{H_{2} O, c}^{\text {in }}+\dot{N}_{H_{2} O, C D L}=\dot{N}_{H_{2} O, c}^{\text {out }}
\end{gathered}
$$

For the anode compartment, the same approach yields:

$$
\begin{gathered}
\dot{N}_{H_{2}, a}^{i n}-\dot{N}_{H_{2}, A D L}=\dot{N}_{H_{2}, a}^{o u t} \\
\dot{N}_{H_{2} O, a}^{i n}-\dot{N}_{H_{2} O, A D L}=\dot{N}_{H_{2} O, a}^{o u t}
\end{gathered}
$$

\section{Average Gas Channel Gas Composition}

The above equations can then be used to determine the average molar flow rates (or average molar fractions) for the different species. Taking the arithmetic mean value of the inlet and outlet values, yields for the cathode compartment:

$$
\begin{gathered}
\overline{\dot{N}}_{O_{2}}=0.5\left(2 \dot{N}_{O_{2}}^{i n}-\dot{N}_{O_{2}, C D L}\right) \\
\dot{\hat{N}}_{H_{2} O, c}=0.5\left(2 \dot{N}_{H_{2} O, c}^{i n}+\dot{N}_{H_{2} O, C D L}\right) \\
\dot{\hat{N}}_{N_{2}}=\dot{N}_{N_{2}}^{i n}
\end{gathered}
$$

For the anode a similar development results in:

$$
\begin{gathered}
\dot{\dot{N}}_{H_{2}}=0.5\left(2 \dot{N}_{H_{2}}^{i n}-\dot{N}_{H_{2}, A D L}\right) \\
\overline{\dot{N}}_{H_{2} O, a}=0.5\left(2 \dot{N}_{H_{2} O, a}^{i n}-\dot{N}_{H_{2} O, A D L}\right)
\end{gathered}
$$

Using the stoichiometric oxygen and hydrogen flow rates along with the water fluxes $\dot{N}_{H_{2} O, C D L}$ and $\dot{N}_{H_{2} O, A D L}$ expressed as a function of $\alpha^{16}$, (2.47) to (2.51) can be rearranged to yield for the cathode:

$$
\overline{\dot{N}}_{O_{2}}=\left(\lambda_{O_{2}}-0.5\right) A \frac{I^{\prime \prime}}{4 F}
$$

\footnotetext{
${ }^{16}$ See chapter 2.5.1 for more details on that notation.
} 


$$
\begin{gathered}
\overline{\dot{N}}_{N_{2}}=4 \lambda_{O_{2}} A \frac{I^{\prime \prime}}{4 F} \\
\overline{\dot{N}}_{H_{2} O, c}=\left(\frac{5 \chi_{H_{2} O, c}^{i n}}{\left(1-\chi_{H_{2} O, c}^{i n}\right)} \lambda_{O_{2}}+1+\alpha\right) A \frac{I^{\prime \prime}}{4 F}
\end{gathered}
$$

And for the anode, a similar development yields:

$$
\begin{gathered}
\overline{\dot{N}}_{H_{2}}=\left(\lambda_{H_{2}}-0.5\right) A \frac{I^{\prime \prime}}{2 F} \\
\overline{\dot{N}}_{H_{2} O, a}=\left(\frac{\chi_{H_{2} O, a}^{i n}}{\left(1-\chi_{H_{2} O, a}^{i n}\right)} \lambda_{H_{2}}-\frac{1}{2} \alpha\right) A \frac{I^{\prime \prime}}{2 F}
\end{gathered}
$$

These average molar flow rates in the anode and cathode compartments can then be used to determine the average molar gas fractions. Substituting (2.35) into (2.52) to (2.56) then yields for the molar gas fractions in the cathode:

$$
\begin{aligned}
\bar{\chi}_{H_{2} O, C G C} & =\frac{\frac{\chi_{H_{2} O, c}^{i n}}{1-\chi_{H_{2} O, c}^{i n}} 5 \lambda_{O_{2}}+\alpha+1}{\left(1+\frac{\chi_{H_{2} O, c}^{i n}}{1-\chi_{H_{2} O, c}^{i n}}\right) 5 \lambda_{O_{2}}+\alpha+0.5} \\
\bar{\chi}_{O_{2}, C G C} & =\frac{\lambda_{O_{2}}-0.5}{\left(1+\frac{\chi_{H_{2} O, c}^{i n}}{1-\chi_{H_{2} O, c}^{i n}}\right) 5 \lambda_{O_{2}}+\alpha+0.5} \\
\bar{\chi}_{N_{2}, C G C} & =\frac{4 \lambda_{O_{2}}}{\left(1+\frac{\chi_{H_{2} O, c}^{i n}}{1-\chi_{H_{2} O, c}^{i n}}\right) 5 \lambda_{O_{2}}+\alpha+0.5}
\end{aligned}
$$

For the anode, a similar development yields:

$$
\bar{\chi}_{H_{2} O, A G C}=\frac{\frac{\chi_{H_{2} O, c}^{i n}}{1-\chi_{H_{2} O, a}^{i n}} \lambda_{H_{2}}-\frac{1}{2} \alpha}{\left(1+\frac{\chi_{H_{2} O, a}^{i n}}{1-\chi_{H_{2} O, a}^{i n}}\right) \lambda_{H_{2}}-\frac{1}{2} \alpha-0.5}
$$




$$
\bar{\chi}_{H_{2}, A G C}=\frac{\lambda_{H_{2}}-0.5}{\left(1+\frac{\chi_{H_{2} O, a}^{i n}}{1-\chi_{H_{2} O, a}^{i n}}\right) \lambda_{H_{2}}-\frac{1}{2} \alpha-0.5}
$$

where $\chi_{\mathrm{H}_{2} \mathrm{O}}^{i n}$ is a function of temperature, gas pressure and relative inlet humidity and is given by:

$$
\chi_{H_{2} O}^{i n}=\frac{\psi p_{s a t}(T)}{p_{G}}
$$

\subsubsection{Gas Transport in the Gas Diffusion Layer}

From the average oxygen and hydrogen molar fractions determined in section 2.4.2, gas transport across the GDL can now be described. Assuming steady state behaviour, the overall oxygen and hydrogen flow rate is directly proportional to the current drawn from the fuel cell according to Faraday's Law (2.24).

The transfer of reaction gases from the gas channel to the reaction site involves three distinct transport mechanisms also shown on Figure 2.8

1. The gas migrates from the gas channel (convection driven) into the gas diffusion layer (diffusion driven). Such a process is typically described by a Sherwood ${ }^{17}$ analogy.

2. The gas diffuses from the GDL region adjacent to the gas channel towards the catalyst layer. This is described as a simple 1D diffusion process.

3. The gas dissolves into the membrane phase-water mixture covering the reaction site. This is described by a Henry ${ }^{18}$ dissolution law.

The governing equations for the different transport mechanisms will be outlined more in detail below. They are given for the case of oxygen

\footnotetext{
${ }^{17}$ The Sherwood number (Sh) is a dimensionless number used to describe mass-transfer. It represents the ratio of length scale to the diffusive boundary layer thickness and can be regarded as the mass transfer analogue of the Nusselt Number $(\mathrm{Nu})$.

${ }^{18}$ The Henry constant describes the ratio of a specie's partial gas pressure to its corresponding concentration when dissolved in a liquid. See Appendix B.2 for more details.
} 
transport in the cathode compartment. However, the same mechanisms apply to hydrogen transport on the anode side.

Note that while multicomponent diffusion is typically described by the Maxwell Equation (B.11), this can be modified to result in a Fick type diffusion with an adapted overall diffusion coefficient based on the composition of the gas mixture (see Appendix B.4.2).

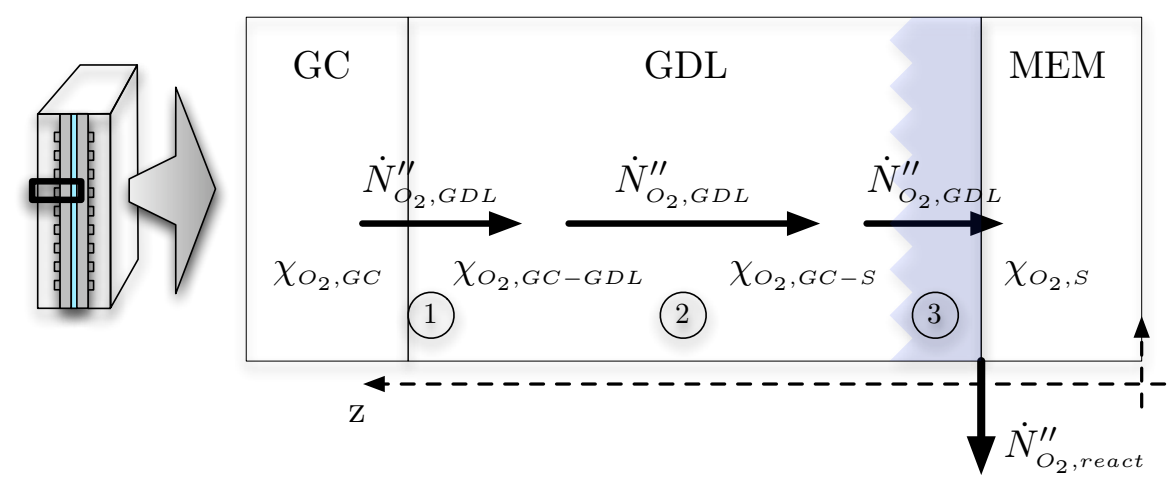

Figure 2.8: Schematic representation of the reaction gas transport in the z-direction perpendicular to the membrane, shown here for the case of oxygen. The same mass transport mechanisms are responsible for hydrogen transport in the anode compartment.

The Sherwood analogy allows to determine the mass transfer from a gas channel into the adjacent GDL by means of a gas concentration at the GDL-GC interface. This concentration can be determined according to:

$$
c_{O_{2}, G C-G D L}=c_{O_{2}, G C}-\frac{\dot{N}_{O_{2}}^{\prime \prime} d_{h} A_{G D L}}{S h D_{O_{2} L, G C} A_{G C}}
$$

Note the area correction factor that takes into account the reduced surface area for diffusion into the GC (only the GC surface is available as opposed to the active membrane surface in the GDL). Also, since the Sherwood number concerns the diffusive boundary layer thickness and because the molar flux rate is expressed as an area specific term, the hydraulic diameter $d_{h}$ represents the hydraulic diameter of one of $n$ parallel gas channels.

For a given oxygen concentration at the GDL-GC interface $c_{O_{2}, G C-G D L}$, the oxygen concentration at the GDL-catalyst layer interface $c_{\mathrm{O}_{2}, G D L-S}$ 
is determined for unidirectional (z-direction) diffusional gas transport according to:

$$
c_{O_{2}, G D L-S}=c_{O_{2}, G C-G D L}-\frac{\delta_{G D L} \dot{N}_{O_{2}}^{\prime \prime}}{D_{O_{2} L, G D L} \phi^{1.5}}
$$

where $\phi^{1.5}$ is a Bruggemann correction factor accounting for the porous nature of the GDL.

Finally assuming liquid water within the catalyst layer, the concentration of dissolved oxygen therein can be written according to Henry's law (see Appendix B.2 for more details).

$$
c_{O_{2}, S}=\frac{p_{O_{2}, G D L-S}}{H_{O_{2}-H_{2} O, e q}}=\frac{c_{O_{2}, G D L-S} \mathcal{R} T}{H_{O_{2}-H_{2} O, e q}}
$$

In order to account for the amount of liquid water present in the catalyst layer, the equivalent Henry constant $\mathrm{H}_{\mathrm{O}_{2}-\mathrm{H}_{2} \mathrm{O} \text {,eq }}$ is expressed as a function of the average saturation ratio $\varphi$ in the gas channel. It is thus given by:

$$
H_{\mathrm{O}_{2}-\mathrm{H}_{2} \mathrm{O}, \mathrm{eq}}=\mathcal{R} T+\varphi \cdot \mathrm{H}_{\mathrm{O}_{2}-\mathrm{H}_{2} \mathrm{O}}
$$

If no water is present in the gas channel, $H_{\mathrm{O}_{2}-\mathrm{H}_{2} \mathrm{O}, e q}=\mathcal{R} T$ and no Henry losses occur. Combining (2.65) with (2.63) and (2.64) yields a description of the dissolved oxygen concentration at the reaction site.

$$
\begin{aligned}
c_{O_{2}, S}=\frac{\mathcal{R} T}{H_{O_{2}-H_{2} O, e q}} & \left(c_{O_{2}, G C}-\frac{\dot{N}_{O_{2}}^{\prime \prime} d_{h} A_{G D L}}{S h D_{O_{2} L, G C} A_{G C}}\right. \\
& \left.-\frac{\delta_{G D L} \dot{N}_{O_{2}}^{\prime \prime}}{D_{O_{2} L, G D L} \phi^{1.5}}\right)
\end{aligned}
$$

\section{Equivalent Diffusion Coefficient}

Alternatively, (2.67) can be written as a function of an equivalent overall GDL diffusivity $D_{e q, G D L}$ which yields:

$$
c_{\mathrm{O}_{2}, S}=\frac{\mathcal{R} T}{H_{\mathrm{O}_{2}-\mathrm{H}_{2} \mathrm{O}}}\left(c_{\mathrm{O}_{2}, G C}-\frac{\delta_{G D L}}{D_{e q, G D L}} \dot{N}_{\mathrm{O}_{2}}^{\prime \prime}\right)
$$


Where the equivalent overall GDL diffusivity is given by:

$$
D_{e q, G D L}=\left(\frac{d_{h} A_{G D L}}{S h \delta_{G D L} D_{O_{2} L, G C} A_{G C}}+\frac{1}{D_{O_{2} L, G D L} \phi^{1.5}}\right)^{-1}
$$

Note that the diffusivities $D_{O_{2} L}^{G C}$ and $D_{O_{2} L}^{G D L}$ are functions of temperature and gas compositions. In order to correctly describe the transport phenomena, the diffusivities would have to be determined for each node in a finite element code.

Since such an approach is not feasible within the framework of an analytical model, the diffusivities are based on the average gas composition in the gas channel (see section 2.4.2) at the given cell temperature. Thus $D_{O_{2} L, G D L}=D_{O_{2} L, G C}$ and therefore the overall equivalent cathode diffusion layer (CDL) diffusivity can be expressed as:

$$
D_{e q, C D L}=\frac{D_{O_{2} L, C G C}}{\frac{d_{h} A_{C D L}}{S h \delta_{C D L} A_{C G C}}+\frac{1}{\phi^{1.5}}}
$$

For the anode diffusion layer (ADL), a similar approach yields:

$$
D_{e q, A D L}=\frac{D_{H_{2} L, A G C}}{\frac{d_{h} A_{A D L}}{S h \delta_{A D L} A_{A G C}}+\frac{1}{\phi^{1.5}}}
$$

Note that in both cases only the nominator is dependent on the actual operating point of the fuel cell - the denominator is characteristic of the system's dimensions and materials.

The dependence of $D_{O_{2} L, C G C}$ and $D_{H_{2} L, A G C}$ on gas composition, temperature and pressure is shown in (B.15) in Appendix B.4.2. In order to keep this dependence on operating parameters, while permitting parameter identification for the diffusive mass transport, $k_{\mathrm{O}_{2}-\mathrm{H}_{2} \mathrm{O}}$ is considered to be a modelling parameter. $k_{\mathrm{O}_{2}-\mathrm{N}_{2}}$ is calculated based on the theoretical quotient $\frac{k_{\mathrm{O}_{2}-\mathrm{H}_{2} \mathrm{O}}}{k_{\mathrm{O}_{2}-\mathrm{N}_{2}}}$.

(2.68) is useful in determining the limiting current density of the fuel cell for a set of operating conditions. A limiting current density is said to occur when the reactant concentration (i.e. oxygen) at the catalyst layer becomes zero. For this to happen, the following relation has to hold:

$$
c_{\mathrm{O}_{2}, G C}=\frac{\delta_{G D L}}{D_{e q, G D L}} \dot{N}_{\mathrm{O}_{2}, l i m}^{\prime \prime}
$$


Incorporating (2.24), this can be solved to yield the limiting current density as shown in (A.20).

$$
I_{l i m}^{\prime \prime}=4 F c_{O_{2}, G C} \frac{D_{e q, G D L}}{\delta_{G D L}}
$$

\subsection{Water Transport}

Water management is one of the critical aspects of successful FC operation. As shown in section 2.3.4, the membrane resistance to ion transfer is strongly dependent on water content. For best conductivity, the membrane should be kept fully humidified which implies high water content in the anode and cathode gas streams.

However, high water contents in the gas streams will lower the cell potential since the oxygen and hydrogen concentrations decrease and more importantly - will increase the danger of water condensation in the GDL or gas channels. When condensation occurs, reaction gas transport pathways become partially or fully blocked and cell potential decreases substantially. If such a blockage is sustained over longer periods of time, inverse cell potentials with resulting permanent membrane damage will follow.

It is therefore important to be able to correctly describe the membrane water content and the mechanisms governing water transport to and from the membrane. The difficulty lies in the fact that water is the only species within an FC that can move throughout the system, i.e. from anode to cathode and back, and is susceptible to condensation.

This chapter proposes a simplified, analytical expression for overall water transport within the fuel cell. First, a brief reminder of the mass balance based average gas channel water concentration is given. To ease the description of the different water fluxes, a water transfer coefficient $\alpha$ is introduced.

Simplified descriptions are then derived from literature to form the governing equations that quantitatively describe the water transport across the GDL and the membrane. Finally, the mass balance approach and the transport phenomena are coupled to yield a description of the overall water transport.

The traditional description of overall water transport necessitating numerical iteration procedures to find a solution will be outlined. In a final 
section, a new approach to obtaining an analytical description for the overall water transfer within the fuel cell will be presented.

\subsubsection{Water Transfer Coefficient $\alpha$}

In order to simplify the description of the water fluxes within the cell, a membrane water transfer coefficient $\alpha$ [43] is introduced measuring the water crossing from the anode to the cathode compartment as a function of the water production at the cathode.

$$
\alpha=\frac{\dot{N}_{H_{2} O, M E M}^{\prime \prime}}{\dot{N}_{H_{2} O, \text { react }}^{\prime \prime}}=\frac{\dot{N}_{H_{2} O, M E M}^{\prime \prime}}{\frac{I^{\prime \prime}}{2 F}}
$$

Through a mass balance on the (AM) and (CM) nodes, the different contributions to water transport shown in Figure 2.9 can be expressed as:

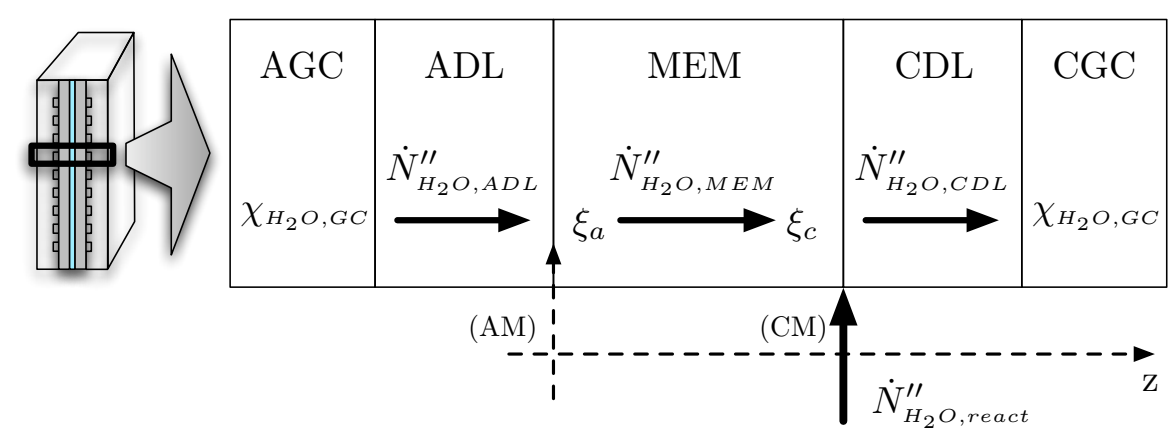

Figure 2.9: Water transport across the GDL-membrane-GDL sandwich within a fuel cell.

$$
\begin{gathered}
\dot{N}_{H_{2} O, A D L}^{\prime \prime}=\dot{N}_{H_{2} O, M E M}^{\prime \prime} \\
\dot{N}_{H_{2} O, \text { react }}^{\prime \prime}+\dot{N}_{H_{2} O, M E M}^{\prime \prime}=\dot{N}_{H_{2} O, C D L}^{\prime \prime}
\end{gathered}
$$

Using (2.74), the node equations (2.75) and (2.76) can be expressed as a function of $\alpha$. Rearranging the result then yields the water fluxes in the anode and cathode gas diffusion layers. 


$$
\begin{gathered}
\dot{N}_{H_{2} O, C D L}^{\prime \prime}=(1+\alpha) \frac{I^{\prime \prime}}{2 F} \\
\dot{N}_{H_{2} O, A D L}^{\prime \prime}=\alpha \frac{I^{\prime \prime}}{2 F}=\dot{N}_{H_{2} O, M E M}^{\prime \prime}
\end{gathered}
$$

\subsubsection{Average Gas Channel Water Concentration}

In section 2.4.2, expressions for the average species concentration (or molar fractions) were obtained from mass balance considerations on both gas channels. The resulting equations ((2.60) and (2.57)) are given as a function of the inlet water molar fractions (i.e. inlet gas humidity), the stoichiometric flow rates $\lambda$ and the water transfer coefficient $\alpha$. For ease of reference, they have been repeated below.

$$
\begin{gathered}
\bar{\chi}_{H_{2} O, C G C}=\frac{\frac{\chi_{H_{2} O, c}^{i n}}{1-\chi_{H_{2} O, c}^{i n}} 5 \lambda_{O_{2}}+\alpha+1}{\left(1+\frac{\chi_{H_{2} O, c}^{i n}}{1-\chi_{H_{2} O, c}^{i n}}\right) 5 \lambda_{O_{2}}+\alpha+0.5} \\
\bar{\chi}_{H_{2} O, A G C}=\frac{\frac{\chi_{H_{2} O, c}^{i n}}{1-\chi_{H_{2} O, a}^{i n}} \lambda_{H_{2}}-\frac{1}{2} \alpha}{\left(1+\frac{\chi_{H_{2} O, a}^{i n}}{1-\chi_{H_{2} O, a}^{i n}}\right) \lambda_{H_{2}}-\frac{1}{2} \alpha-0.5}
\end{gathered}
$$

The results have been plotted for a given set of temperatures, pressures and relative inlet humidities and are shown in Figure 2.10 and Figure 2.11. The blueish, upper horizontal surface represents the saturation level while the grayish, lower horizontal surface shows the minimum average water content.

(2.79) can be modified to express $\alpha$ as a function of the average molar water fraction in the cathode gas channel. Substituting $k_{c}=\frac{\chi_{\mathrm{H}_{2} O, c}^{i n}}{1-\chi_{\mathrm{H}_{2} \mathrm{O}, c}}$ yields:

$$
\alpha=\frac{5 \lambda_{O_{2}} k_{c}-5 \bar{\chi}_{\mathrm{H}_{2} \mathrm{O}, C G C} \lambda_{c}\left(1+k_{c}\right)-0.5 \bar{\chi}_{\mathrm{H}_{2} O, C G C}+1}{\bar{\chi}_{\mathrm{H}_{2} O, C G C}-1}
$$


$80^{\circ} \mathrm{C}, 50 \% \mathrm{RH}$ and 2 bar(a)

$80^{\circ} \mathrm{C}, 100 \% \mathrm{RH}$ and 2 bar(a)
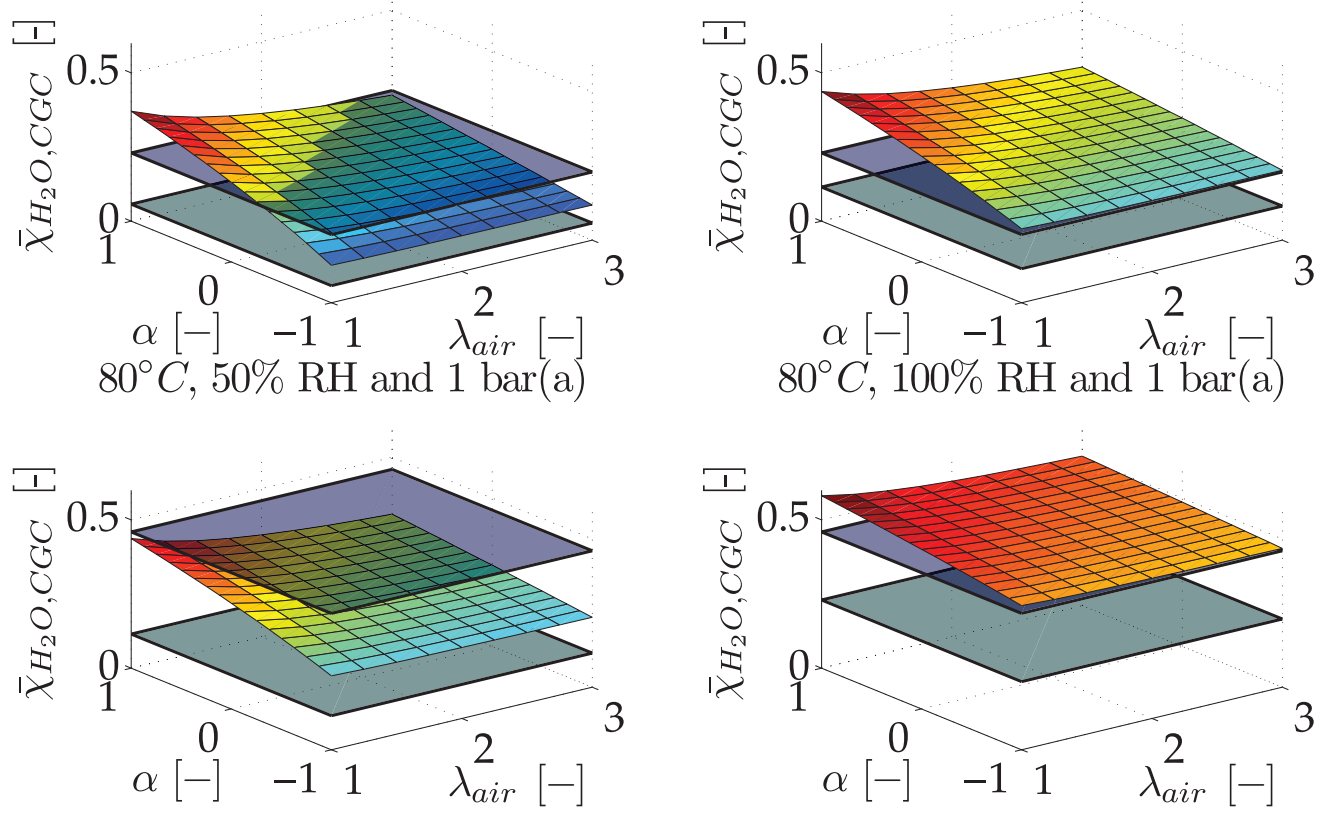

Figure 2.10: Average molar water fraction in the cathode gas channel for varying operating parameters. The blueish, upper horizontal surface represents the saturation level while the grayish, lower horizontal surface shows the minimum average water content. 
$80^{\circ} \mathrm{C}, 50 \% \mathrm{RH}$ and 2 bar(a)
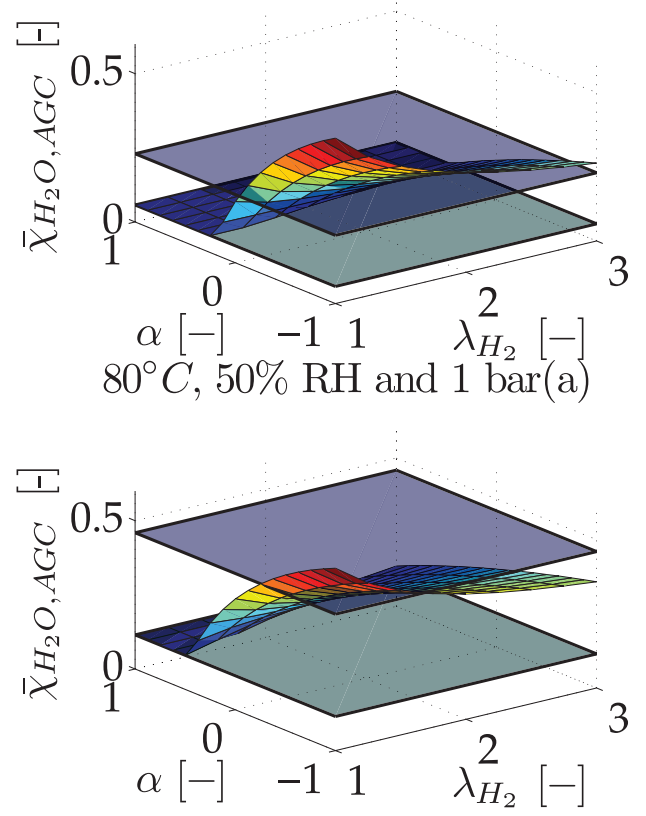

$80^{\circ} \mathrm{C}, 100 \% \mathrm{RH}$ and $2 \operatorname{bar}(\mathrm{a})$
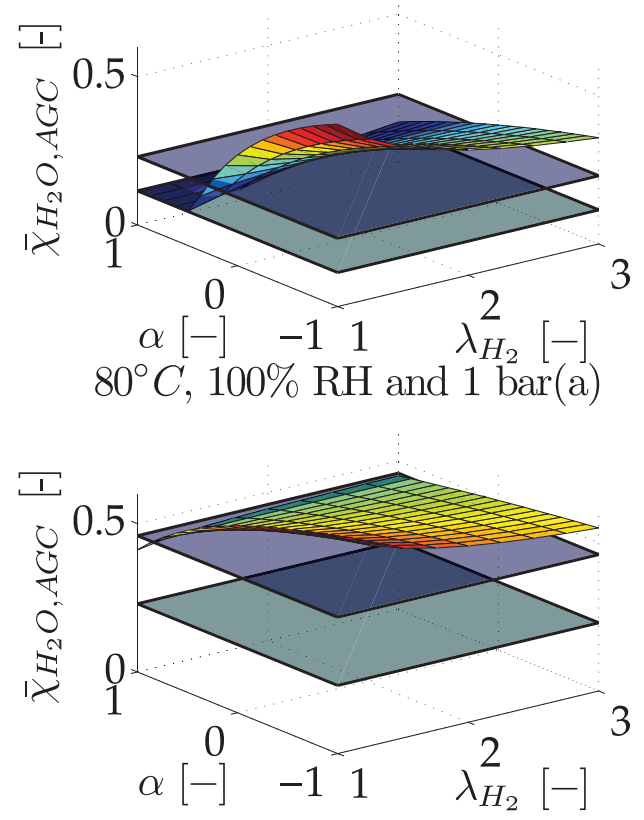

Figure 2.11: Average molar water fraction in the anode gas channel for varying operating parameters. The blueish, upper horizontal surface represents the saturation level while the grayish, lower horizontal surface shows the minimum average water content. 
This can then be inserted into (2.80) to directly relate the average water molar fraction in the anode gas channel to the one in the cathode gas channel.

The minimum average molar water fraction in either channel occurs (mutually exclusively) when all water entering the gas channel is transported across the membrane. The resulting average molar water fraction in that channel then corresponds to half the inlet molar water fraction and is shown as a grayish, horizontal surface on Figure 2.10 and Figure 2.11.

This can be expressed as a corresponding minimum and maximum water transfer coefficient $\alpha=f\left(\chi_{\mathrm{H}_{2} \mathrm{O}}^{i n}, \lambda\right)$ for the cathode and anode respectively and is shown in Figure 2.12.

$$
\begin{gathered}
\alpha \geq-\frac{\chi_{H_{2} O, c}^{i n}}{1-\chi_{H_{2} O, c}^{i n}} \frac{5}{2} \lambda_{O_{2}}-1 \\
\alpha \leq \frac{\chi_{H_{2} O, a}^{i n}}{1-\chi_{H_{2} O, a}^{i n}} \lambda_{H_{2}}
\end{gathered}
$$

Note that the minimum water transfer coefficient occurs when all water entering the cathode gas channel crosses the membrane into the anode compartment along with all water produced by the electrochemical reaction. The maximum water transfer coefficient corresponds to all water entering the anode gas channel crossing to the cathode side.

$$
\text { Cathode: } \alpha_{\text {min }}=\mathrm{f}\left(\lambda_{\text {air }}, \chi_{H_{2} O, C G C}^{i n}\right) \quad \text { Anode: } \alpha_{\max }=\mathrm{f}\left(\lambda_{H_{2}}, \chi_{H_{2} O, A G C}^{i n}\right)
$$

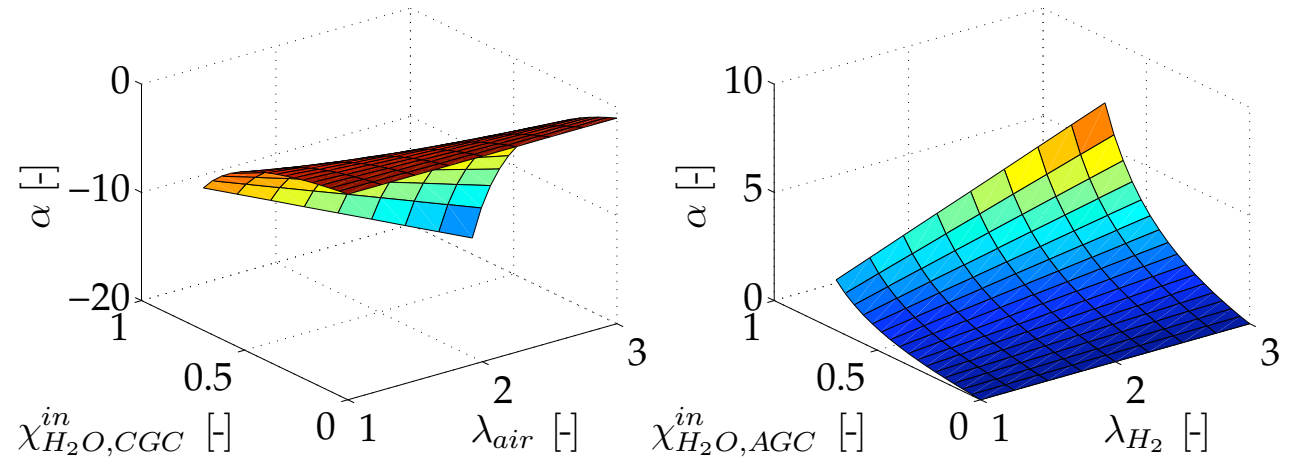

Figure 2.12: Maximum and minimum water transfer coefficients depending on the inlet molar water fraction and the stoichiometric flow rate in the cathode and anode GC respectively. 


\subsubsection{Water Transport within the GDL}

Water transport in the GDL is closely interwoven with gas transport in that same region. In order to correctly describe what is happening, multicomponent diffusion with phase changes and liquid water convection would have to be accounted for [44]. The resulting equations can only be solved by finite element software and will therefore be unsuitable for our purposes.

Berg et al. [45] have shown an interesting approach that allows to regroup most unknowns in one parameter that has to be fitted to experimental data thus providing an analytical expression of water transport within the GDL. Their approach has been adapted and is outlined in the following paragraphs. It consists of using the similarity in governing equations between liquid water transport described by Darcy's law and gaseous species transport described by Fick's law. Appropriate modifications of both governing equations show that both represent the species flux as a resistance term times a difference in potential.

The resistance term of both equations has been regrouped in an overall water transfer resistance coefficient $\gamma$ that describes mass transport limitations due to the GDL and the water uptake of the membrane through the GDL-MEM interface.

As shown in chapter 2.3.4, for equilibrium conditions, membrane water content can be expressed as a function of water activity in the adjacent gas phase. This is then used to define equivalent water content values $\xi_{a}^{*}$ and $\xi_{c}^{*}$ (2.16) based on the water activities in the GC. The difference between actual membrane water content and the equivalent membrane water content based on the gas composition in the GC is then used as a driving force for water transport which can be expressed as follows:

$$
\begin{gathered}
\dot{N}_{H_{2} O, A D L}^{\prime \prime}=-\gamma \frac{\rho}{E W}\left(\xi_{a}-\xi_{a}^{*}\right) \\
\dot{N}_{H_{2} O, C D L}^{\prime \prime}=\gamma \frac{\rho}{E W}\left(\xi_{c}-\xi_{c}^{*}\right)
\end{gathered}
$$

Substituting the water fluxes with their respective expression in $\alpha$ then yields:

$$
\alpha \frac{I^{\prime \prime}}{2 F}=-\gamma \frac{\rho}{E W}\left(\xi_{a}-\xi_{a}^{*}\right)
$$




$$
(1+\alpha) \frac{I^{\prime \prime}}{2 F}=\gamma \frac{\rho}{E W}\left(\xi_{c}-\xi_{c}^{*}\right)
$$

Saturation is said to occur if the membrane water content becomes $\xi=14$. In such a case, $\xi a$ and $\xi c$ are capped at 14 .

For finite element analysis calculations, (2.85) and (2.84) are perfectly adapted since based on boundary conditions at the channel inlet $\left(\xi_{c, \text { in }}^{*}\right.$ and $\left.\xi_{a, i n}^{*}\right)$, the resulting fluxes and water concentrations can be calculated.

Unfortunately, for an overall balance notation, the average water activity in the gas channel $\bar{\chi}_{H_{2} O, G C}$ depends on the water flux across the GDL. Therefore, $\bar{\xi}_{c}^{*}$ and $\bar{\xi}_{a}^{*}$ need to be expressed as a function of water fluxes in the anode and cathode GDLs (see chapter 2.4.2).

\subsubsection{Water Transport within the Membrane}

Water transport in the membrane can be related to three distinct transport phenomena:

Water transport by electroosmotic drag is related to a frictional interaction between protons and water molecules - as protons migrate across the membrane water molecules get dragged along.

Water transport by diffusion (backdiffusion) is due to a water concentration difference on either side of the membrane. Typically, water concentration at the cathode is higher than at the anode due to the water production by the electrochemical reaction. Since the resulting water transport is in the opposite direction of the proton movement, this phenomena is also called backdiffusion.

Water transport by convection is due to a pressure difference across the membrane. This is typically given by the FC system design.

The mechanisms associated with these three ways of water transport will be shown in the following paragraphs. 


\section{Water Transport by Electroosmotic Drag}

Referring to Springer et al. [12], the electroosmotic drag coefficient $n_{\text {drag }}$ is adopted. It is assumed to be proportional to the membrane water content and approximated by:

$$
n_{\text {drag }}=2.5 \frac{\xi}{22}
$$

Including (2.88) in (2.24), the net water flux rate due to electroosmotic drag can be written as:

$$
\dot{N}_{\mathrm{H}_{2} \mathrm{O}, \text { drag }}^{\prime \prime}=n_{\text {drag }} \frac{I^{\prime \prime}}{F}
$$

\section{Water Transport by Diffusion}

Diffusional species transport is governed by Fick's Law (B.9) which can be adapted to be expressed as a function of a membrane water content differential:

$$
\dot{N}_{H_{2} O, d i f}^{\prime \prime}=-\frac{\rho_{d r y}}{E W_{m}} D_{H_{2} O}^{M E M} \frac{d \xi}{d z}
$$

The difficulty then resides in determining the equivalent diffusion coefficient for water.

Motupally et al. [46] have done a review on water diffusion coefficients in literature and reported data from Zawodzinski et al. [47], Fuller [15] and Nguyen et al. [14]. The corresponding equations are shown in (2.91), (2.92), (2.93) and (2.94).

$$
\begin{gathered}
D_{\mathrm{H}_{2} \mathrm{O}}^{M E M}=3.1 \cdot 10^{-7} \xi\left(-1+e^{0.28 \xi}\right) \exp \left[-\frac{2436}{T}\right], \text { for } 0<a \leq 3 \\
D_{\mathrm{H}_{2} \mathrm{O}}^{M E M}=4.17 \cdot 10^{-8} \xi\left(1+161 e^{-\xi}\right) \exp \left[-\frac{2436}{T}\right], \text { for } 3 \leq a<17 \\
D_{\mathrm{H}_{2} \mathrm{O}}^{M E M}=2.1 \cdot 10^{-7} \xi \exp \left(-\frac{2436}{T}\right)
\end{gathered}
$$




$$
D_{\mathrm{H}_{2} \mathrm{O}}^{M E M}=\left(1.76 \cdot 10^{-9}+1.94 \cdot 10^{-8} \xi\right) \exp \left(-\frac{2436}{T}\right)
$$

Additionally, Springer et al. [12] have fitted their experimental data (for values of $\xi \geq 4$ ) to the following relation which was subsequently adopted by Olsommer et al. [48]:

$$
\begin{aligned}
D_{H_{2} \mathrm{O}}^{M E M}= & \left(2.563-0.33 \xi+0.0264 \xi^{2}-0.000671 \xi^{3}\right) 10^{-10} \\
& \exp \left[2416\left(\frac{1}{303}-\frac{1}{T}\right)\right]
\end{aligned}
$$

Apart from Zawodzinski et al.'s discontinous equations (2.91) and (2.92), all of the above expressions for the water diffusion coefficient can be expressed as a function in the form of

$$
D_{\mathrm{H}_{2} \mathrm{O}}^{M E M}=\left(d_{1}+d_{2} \xi+d_{3} \xi^{2}+d_{4} \xi^{3}\right) \exp \left(-\frac{d_{5}}{T}\right)
$$

where the coefficients depend on the chosen curve fit. Table 2.4 lists the different parameters for curve fits from Fuller et al. [15], (2.93), Nguyen et al. [14], (2.94) and Springer et al. [12], (2.95).

\begin{tabular}{cccc}
\hline Curve fit parameters & Fuller et al. & Nguyen et al. & Springer et al. \\
\hline$d_{1}$ & 0 & $1.7 \cdot 10^{-9}$ & $7.44 \cdot 10^{-7}$ \\
$d_{2}$ & $2.1 \cdot 10^{-7}$ & $1.94 \cdot 10^{-8}$ & $-9.58 \cdot 10^{-8}$ \\
$d_{3}$ & 0 & 0 & $7.66 \cdot 10^{-9}$ \\
$d_{4}$ & 0 & 0 & $-1.95 \cdot 10^{-10}$ \\
$d_{5}$ & 2436 & 2436 & 2416 \\
\hline
\end{tabular}

Table 2.4: Coefficients for the general expression of the water diffusion coefficient in the membrane phase according to different experimental data and curve fits.

The experimental values ${ }^{19}$ found by [12] along with the values given by (2.91) to (2.95) are shown in Figure 2.13. Since the temperature correction

\footnotetext{
${ }^{19}$ Note the pronounced peak in experimental data at water contents $\xi \approx 3$. In fact, the experimentally determined diffusivity was an intradiffusion coefficient $D^{\prime}$ relating water diffusion to the gradient of the logarithm of activity (i.e.
} 
factors are virtually independent between the different formulations, only values at $30^{\circ} \mathrm{C}$ have been plotted.

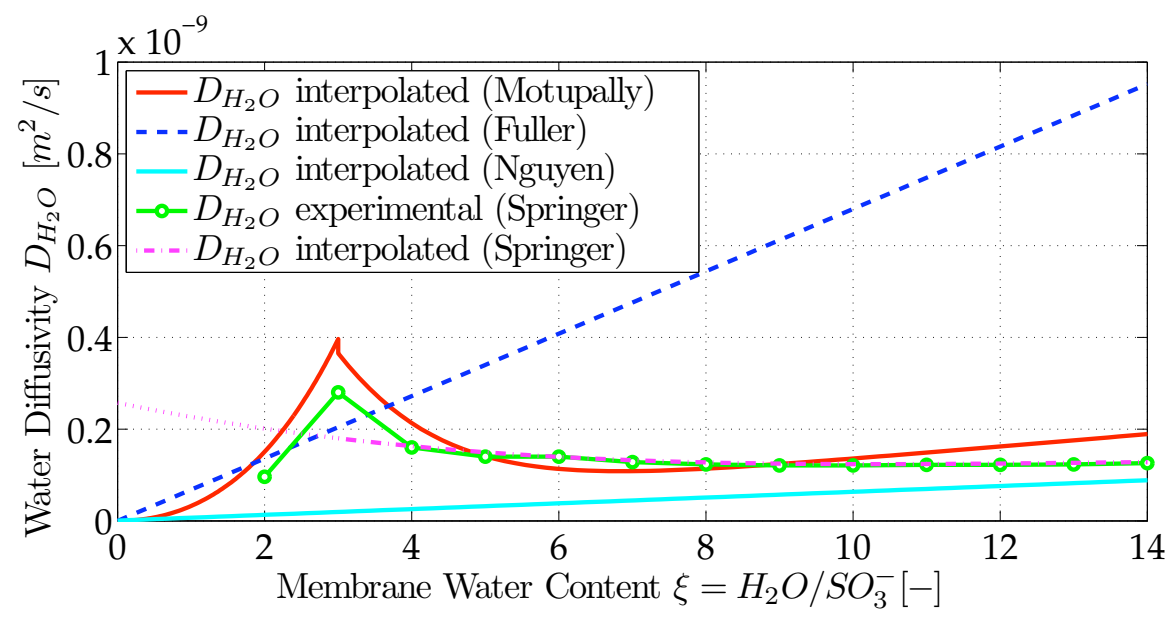

Figure 2.13: Water diffusivity in the membrane region at $30^{\circ} \mathrm{C}$ as a function of membrane water content (Experimental values as determined by [12] and interpolated values according to (2.91) to (2.95)).

Because of the limited membrane conductivity at low humidification conditions, fuel cells need to be operated with high values of $\xi$. Therefore, the peak observed in Figure 2.13 is rarely encountered in practice and is generally neglected by adopting a linear increase of the diffusivity with membrane water content.

Accounting for the large discrepancy of the different formulations, a constant ${ }^{20}$ value for water diffusivity between membrane water contents of $5<\xi \leq 14$ can be regarded as appropriate.

\section{Water Transport by Convection}

Convective water transport is due to a pressure differential across the membrane. Rowe et al. [17] have expressed the resulting water flux in

$\left.\dot{N}_{H_{2} O, d i f}^{\prime \prime}=D^{\prime} c_{H_{2} O} \frac{d(\ln a)}{d \xi} \frac{d \xi}{d z}\right)$. (2.16) was then used to transform the experimentally determined intradiffusion coefficient $D^{\prime}$ into the effective water diffusion coefficient $D_{\mathrm{H}_{2} \mathrm{O}}^{M E M}$. Therefore, the steep gradient of (2.16) around $\xi=3$ makes $D_{\mathrm{H}_{2} \mathrm{O}}^{\mathrm{MEM}}$ very sensitive to the exact form of (2.16) (i.e. its coefficients that have been curve-fitted).

${ }^{20}$ Such a value would still need to be corrected for temperature dependence. The temperature dependence as stated in (2.91) to (2.95) can simply be carried over. 
terms of the hydraulic membrane permeability and water concentration. Their expression can be extended to be a function of membrane water content:

$$
\dot{N}_{H_{2} O, c o n v}^{\prime \prime}=-\frac{\xi \rho_{m, d r y}}{E W_{m}(1+s \xi)} \frac{k_{p}}{\mu_{H_{2} O}} \frac{\Delta p}{\delta_{m}}
$$

Where $s=0.0126$ is a non-dimensional swelling factor experimentally determined by Springer et al. [12]. Note that in (2.97), membrane swelling was assumed to occur in the thickness dimension only. If expansion in the $x-y$ plane is to be included, the cube of the correction term $(1+s \xi)$ needs to be applied.

Typical literature values for the hydraulic membrane permeability are shown in Table 2.5 .

\begin{tabular}{ccc}
\hline $\begin{array}{c}k_{p} \\
{\left[\mathrm{~m}^{2} / s\right]}\end{array}$ & $\begin{array}{c}\mathrm{T} \\
{\left[{ }^{\circ} \mathrm{C}\right]}\end{array}$ & Source \\
\hline $1.8 \cdot 10^{-18}$ & 80 & Bernardi et al. [13] \\
$1.8 \cdot 10^{-18}$ & 90 & Bernardi et al. [13] \\
$1.58 \cdot 10^{-18}$ & 80 & Fales et al. [49] \\
\hline
\end{tabular}

Table 2.5: Typical literature values for the hydraulic membrane permeability $k_{p}$.

\section{Overall Water Transport within the Membrane}

(2.89), (2.90) and (2.97) can be combined to give the overall water transport through the membrane. Notations are further simplified by introducing $e=2.5 \frac{I^{\prime \prime}}{22 F}$ and $f=\frac{\rho_{d r y}}{E W_{m}} \frac{k_{p}}{\mu_{H_{2} O} O} \frac{\Delta p}{\delta_{m}}$.

$$
\begin{aligned}
\dot{N}_{H_{2} O, M E M}^{\prime \prime}= & \dot{N}_{H_{2} \mathrm{O}, d r a g}^{\prime \prime}+\dot{N}_{H_{2} O, d i f}^{\prime \prime}+\dot{N}_{H_{2} O, c o n v}^{\prime \prime} \\
= & 2.5 \frac{\xi}{22} \frac{I^{\prime \prime}}{F}-\frac{\rho_{m}}{E W_{m}} D_{H_{2} O}^{M E M} \frac{d \xi}{d z} \\
& -\frac{\xi \rho_{m}}{E W_{m}(1+s \xi)} \frac{k_{p}}{\mu_{H_{2} O}} \frac{\Delta p}{\delta_{m}} \\
= & -D_{H_{2} O}^{M E M} \frac{d \xi}{d z}+\left(e-\frac{f}{(1+s \xi)}\right) \xi
\end{aligned}
$$


Figure 2.14 gives an idea of the order of magnitude of the water flux rates due to drag-, diffusive- or convective driving forces. The values shown have been established with standard literature values and apply to a Nafion 112 membrane.
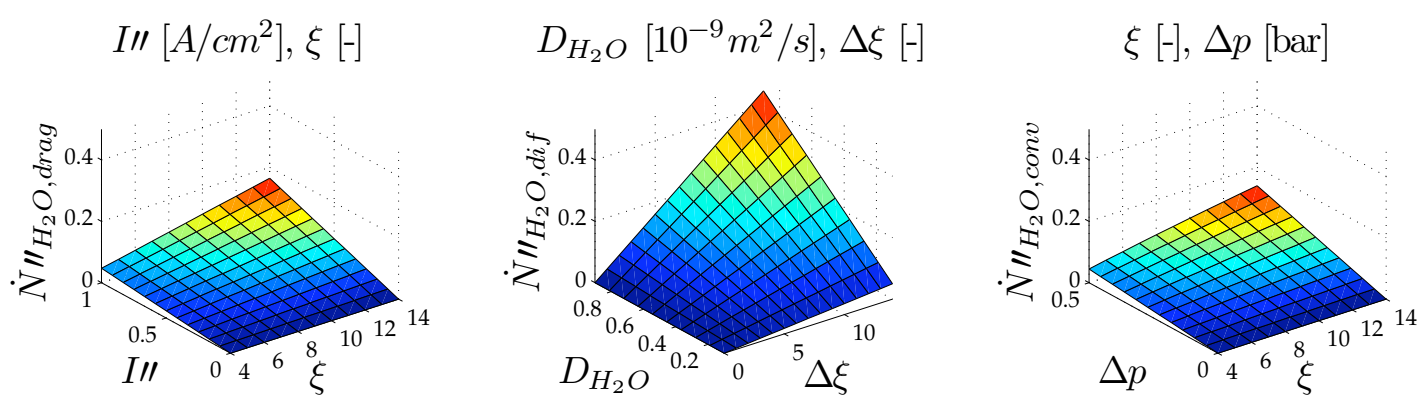

Figure 2.14: Overview of the different contributions (drag, diffusive and convective) to water transport across the membrane. The water flux rates $\left[\frac{m o l}{s \cdot m^{2}}\right]$ have been calculated for standard literature values for the parameters and are shown as a function of the driving force(s). For the case of diffusive transport, the flux rate is also shown as a function of the water diffusivity in the membrane since there are large differences between the values proposed in literature.

(2.98) can then be integrated over the membrane thickness to get the water transfer rate as a function of the water concentration at the membrane interfaces. Unfortunately, there is no direct solution to the differential equation as it is.

\subsubsection{Iterative Approaches to Overall Water Transport within the Fuel Cell}

The overall water transport in the FC can be solved by solving the system of equations formed by the governing equation of all regions - i.e. the gas channel, the ADL and CDL layers along with the membrane region. Unfortunately, there is no direct solution to the equations as they have been shown in sections 2.5.2 to 2.5.4. Appropriate simplifications have to be introduced. This section gives a brief overview of the approaches generally adopted in literature. Some of the expressions presented will form the basis for a non-iterative description of the overall water transport within the fuel cell. 


\section{The Berg et al. Approach}

The Berg et al. [45] approach for the membrane region consists in neglecting water transport due to pressure differences and considering electroosmotic drag independent of membrane water content. Additionally, the water diffusivity in the membrane is considered a linear function of water content. Substituting $\dot{N}_{H_{2} O, M E M}^{\prime \prime}=\alpha \frac{I^{\prime \prime}}{2 F}$, this results in (2.98) being reduced to:

$$
\alpha \frac{I^{\prime \prime}}{2 F}=-d_{2} \exp \left(-\frac{d_{5}}{T}\right) \xi \frac{d \xi}{d z}+\frac{I^{\prime \prime}}{F}
$$

(2.99) can be solved to yield an expression for the water transfer coefficient $\alpha$ as a function of the equivalent water sorption values in the gas channel $\left(\xi_{a}^{*}\right.$ and $\left.\xi_{c}^{*}\right)$ :

$$
\alpha=\frac{-\frac{h^{2}+2 h \xi_{c}^{*}+\xi_{c}^{* 2}-\xi_{a}^{* 2}}{2 \delta_{m}}+\frac{\frac{I^{\prime \prime}}{F}}{d_{2} \exp \left(-\frac{d_{5}}{T}\right)}}{\frac{2 h\left(\xi_{c}^{*}+\xi_{a}^{*}\right)}{2 \delta_{m}}+\frac{I^{\prime \prime}}{2 F d_{2} \exp \left(-\frac{d_{5}}{T}\right)}}
$$

where $h=\frac{I^{\prime \prime} E W}{2 F \gamma \rho}$. (2.99) can then be solved to yield an analytical expression of membrane water content as a function of the position in the membrane. This can then be integrated to result in an average membrane water content that can be used to determine the membrane conductivity. More details can be found in [45].

While (2.100) provides an analytical expression for $\alpha$ for a known gas composition in the anode and cathode gas channels (i.e. known $\xi_{c}^{*}$ and $\xi_{a}^{*}$ ), no overall analytical expression can be found to express $\alpha$ as a function of the cell's operating conditions. Numerical methods have to be used to find an overall solution.

\section{The Linear Concentration Profile Approach}

The Linear Concentration Profile Approach consists in assuming a linear water content variation (i.e. a constant gradient) within the membrane and expressing all concentration dependent variables as a function of the 
average water content. In the CDL, this linear water profile assumption is introduced to replace $\xi_{c}$ with $2 \bar{\xi}-\xi_{a}$. This yields:

$$
(1+\alpha) \frac{I^{\prime \prime}}{2 F}=\gamma \frac{\rho}{E W}\left(2 \bar{\xi}-\xi_{a}-\xi_{c}^{*}\right)
$$

For the ADL region, (2.86) can be solved for $\xi_{a}$, which can then be substituted into (2.101). This results in an expression for the average membrane water content.

$$
\bar{\xi}=\frac{1}{2} \frac{I^{\prime \prime}}{2 F} \frac{E W}{\gamma \rho}+\frac{1}{2}\left(\xi_{c}^{*}+\xi_{a}^{*}\right)
$$

For the membrane region, the governing equation (2.98) can be rewritten with the linear water profile assumption to yield:

$$
\alpha \frac{I^{\prime \prime}}{2 F}=-D \frac{2 \bar{\xi}-\xi_{a}}{\delta_{m}}+\left(e-\frac{f}{(1+s \bar{\xi})}\right) \bar{\xi}
$$

Substituting $\xi_{a}$ with (2.86) then allows to simplify (2.103) to result in an expression for $\alpha$ as a function of $\bar{\xi}$.

$$
\alpha=\frac{\left(e+\frac{f}{(1+s \bar{\xi})}-\frac{2 D}{\delta_{m}}\right) \bar{\xi}+\frac{D}{\delta_{m}} \xi_{a}^{*}}{\frac{I^{\prime \prime}}{2 F}\left(1+\frac{D}{\delta_{m}} \frac{E W}{\gamma \rho}\right)}
$$

The overall water transfer coefficient $\alpha$ can then simply be calculated as a function of the equivalent water sorption values in the gas channel $\left(\xi_{a}^{*}\right.$ and $\xi_{c}^{*}$ ) by substituting (2.102) into (2.104). Since $D$ is also a function of $\bar{\xi}$, this results in a rather complex expression and is therefore not shown here.

As with the approach outlined in section 2.5.5, (2.104) cannot be solved analytically to yield an expression for $\alpha$ as a function of the cell's operating conditions. It is therefore only useful in iterative procedures trying to converge towards a water transfer coefficient based on mass transfer and mass balance considerations. 


\subsubsection{Analytical Expression for the Overall Water Transport}

In order to obtain an analytical solution to the overall water balance, additional simplifications have to be introduced into the governing equations.

- The membrane water sorption characteristic (see chapter 2.3.4) is considered linear. This can be expressed as:

$$
\xi^{*}=a+b \bar{\chi}
$$

- The water diffusivity in the membrane phase is considered constant. This is justified by the large discrepancies (see section 2.5.4) between experimental data and the fact that fuel cells operate in a high membrane water content regime where experimental data suggests an almost constant diffusivity. It leads to a water diffusivity formulation of the form:

$$
D_{H_{2} O, M E M}=d_{1} \cdot \exp \left(\frac{d_{5}}{T}\right)=d_{6}
$$

- The swelling factor $s$ in the term related to convective water transfer (2.97) is considered zero.

- As in section 2.5.5, the water content profile within the membrane is assumed linear. The average membrane water content is then given by:

$$
\bar{\xi}=\frac{1}{2} \frac{I^{\prime \prime}}{2 F} \frac{E W}{\gamma \rho}+\frac{1}{2}\left(\xi_{c}^{*}+\xi_{a}^{*}\right)=k+\frac{1}{2}\left(\xi_{c}^{*}+\xi_{a}^{*}\right)
$$

Accounting for the above simplification results in a governing equation for water transport in the membrane, one for water transport in the gas diffusion layer and the mass balance equations for the anode and cathode gas channels. In order to simplify subsequent calculations, a number of substitutions has been undertaken to express the different governing equations. The substitutions along with the solution procedure can be found in Appendix C.1. 
The approach finally results in an equation for the overall water transfer coefficient $\alpha$.

$$
\begin{aligned}
\alpha= & \frac{t}{s}\left(k+a+0.5 \frac{b(n-0.5 \alpha)}{n+q-0.5 \alpha-0.5}+0.5 \frac{b(m+\alpha+1)}{m+p+\alpha+0.5}\right) \\
& +\frac{u}{s}\left(a+\frac{b(n-0.5 \alpha)}{n+q-0.5 \alpha-0.5}\right)
\end{aligned}
$$

This can then be expanded into a cubic equation for the water transfer coefficient $\alpha$ as a function of the system parameters and operating conditions.

$$
0=a_{0}+a_{1} \alpha+a_{2} \alpha^{2}+a_{3} \alpha^{3}
$$

The coefficients in (2.109) are given in Appendix C.1. Since the equation for $\alpha$ is cubic, there can be 1,2 or 3 real solutions. The mass balance equations - for which no simplifying assumptions have been made - are used to delimit the solution interval and determine the correct solution. If several - from a mass balance point of view valid - solutions are found, the trend in previous time steps is used to determine the correct solution.

(2.109) represents a fully analytical solution to the overall water transfer problem in a fuel cell.

\subsection{Overall Fuel Cell Stack Model}

Sections 2.2 to 2.5 have introduced the building blocks for a steady state, cell-averaged, analytical PEM fuel cell stack model that predicts the fuel cell voltage and membrane water content based on the operating conditions (see Table 2.1). This section outlines how those building blocks fit together to form the overall stack model as shown in Figure 2.15.

In a first step, based on the operating conditions, the water transfer coefficient $\alpha$ is calculated by solving (2.109). With the given water transfer coefficient, the average species concentrations $\bar{\chi}_{i}$ in the gas channel are determined according to (2.57) to (2.61)

With $\alpha$ and $\bar{\chi}_{i}$, the average membrane water content $\bar{\xi}$ and the partial reactant pressures at the reaction sites can be determined according to (2.107) and (2.68) respectively. 


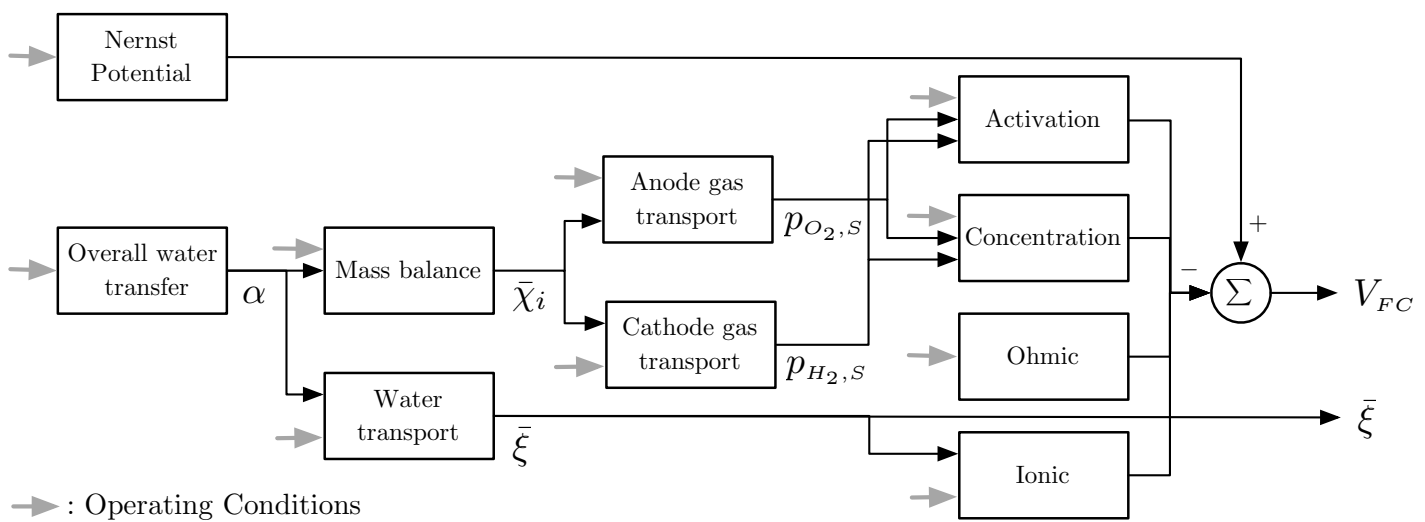

Figure 2.15: Flowchart outlining the fuel cell stack model elaborated in chapter 2 .

In a final step, the performance equations (2.1), (2.4), (2.11), (2.12), (2.14) and (2.23) are evaluated based on the average membrane water content $\bar{\xi}$ and the partial reactant pressures at the reaction sites $p_{i, S}$ to yield the overall cell voltage.

The dependence of the different variables on the operating conditions is shown in Table 2.6. A variable that depends directly on a given operating condition is denoted with "๑". A variable that is calculated based on another one which itself - directly or indirectly - depends on the considered operating condition is noted "o".

\subsection{Summary and Outlook}

In the first part of this chapter, the equations describing fuel cell performance have been introduced. They were mainly adapted from descriptions available in literature. In a second part, an approach for averaging the species concentration over the length of the gas channel was introduced. These averaged species concentrations then formed the basis for a 1 dimensional description of reaction gas and water transport.

A novel approach for analytically determining the overall water transport within the fuel cell based on operating conditions was introduced. This eliminates the traditional iterative procedure to determine water transport across the cell. 


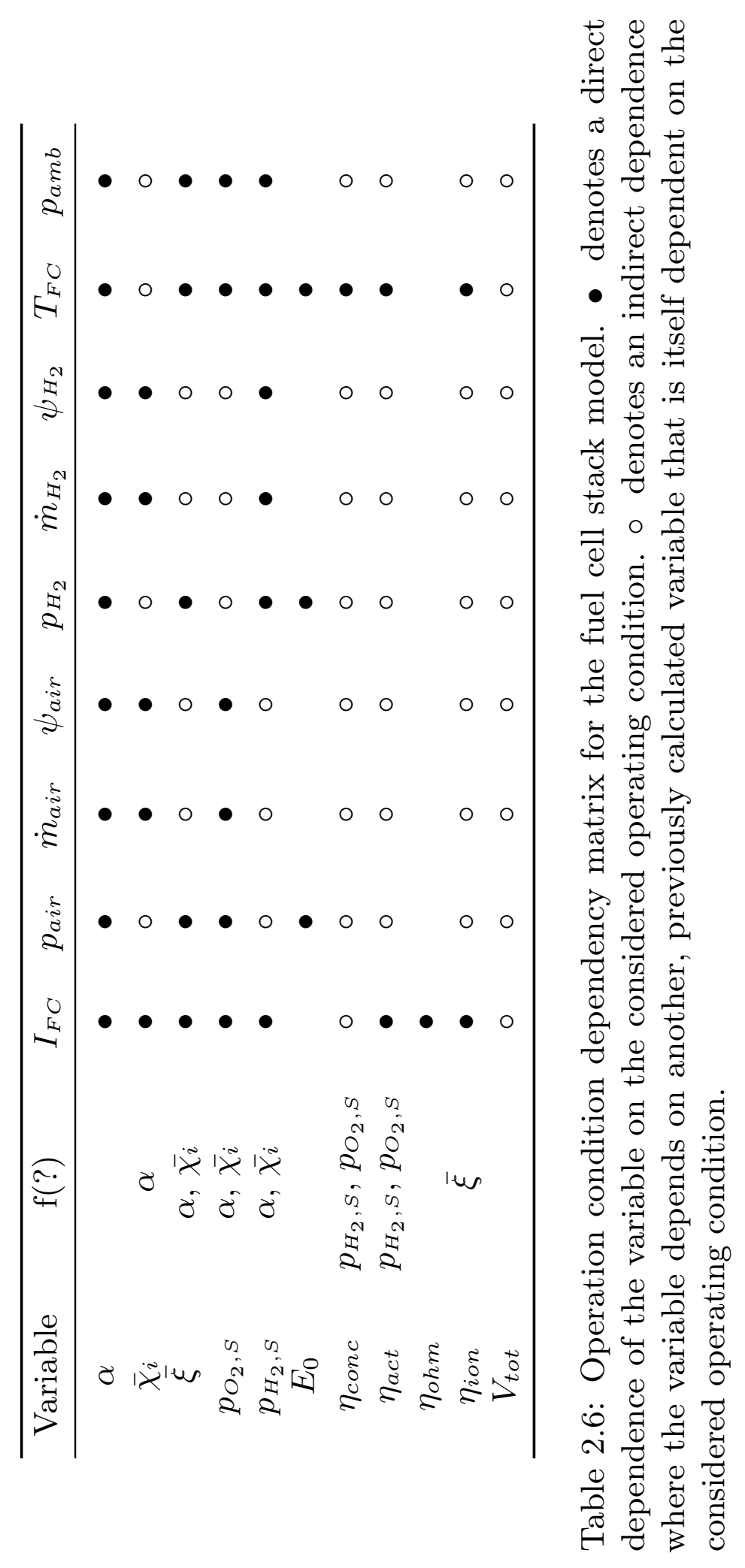


Finally, the modelling equations presented within this chapter are assembled to form an analytical, cell-averaged, steady state fuel cell stack model that predicts the stack voltage along with the membrane water content based on the operating conditions of the fuel cell.

The model can be adjusted for different PEM fuel stacks by fitting certain parameters of the model (see chapter 5 for more details).

The resulting model now forms the basis of the desired fuel cell system model. In chapter 3, dynamic, state-space models are introduced for the different auxiliary systems. Those models then provide the linkage that connect the user's interaction with the system (e.g. applying a certain voltage to the air compressor) to an operating condition for the fuel cell stack (e.g. air mass flow rate).

Once they are assembled, the effect of a user's interaction with the system will be directly described as a resulting modification in fuel cell voltage and membrane water content. 
Balance of Plant Models 


\subsection{Introduction}

This section focuses on modelling the auxiliary systems that supply the fuel cell with reactant gases at appropriate flow rates, pressures and humidities and control the temperature of the cell. They are the link between the user (or ultimately a system controller) and the operating conditions of the fuel cell stack. Understanding and modelling their static and dynamic behaviour therefore provides a description of how user actions affect overall system performance.

In order to easily integrate the subsystems into an overall fuel cell system model, the focus is to derive a linear (or linearised) state space subsystem model in matrix notation.

A first section focuses on describing the air supply subsystem resulting in a dynamic description of the mechanical, electrical and hydraulic phenomena therein. Additional elements that could - in a different fuel cell system - be part of the air supply system are explained and their modelling approach outlined.

The hydrogen supply subsystem is then described and modelled. Even though the hydrogen pressure cannot be adjusted dynamically by the user, a description of the mechanical pressure regulator is given to provide an understanding of the involved mechanisms.

A lumped capacitance model of the thermal management subsystem is developed to describe the temperature dynamics of the system based on user and system inputs. The resulting model is non-linear and needs to be linearised around a given operating point.

\subsection{System Description}

The FC system considered here is shown in Figure 3.1. It is composed of an air and hydrogen supply and conditioning system, a thermal management subsystem along with the fuel cell stack.

In order to facilitate the understanding of the modelling approach, a brief overview of the functions assumed by the different subsystems will be given.

A volumetric air compressor feeds air into the cathode manifold where it passes through a humidification system and enters the FC stack at a given temperature and relative humidity. From the cathode, where part of 


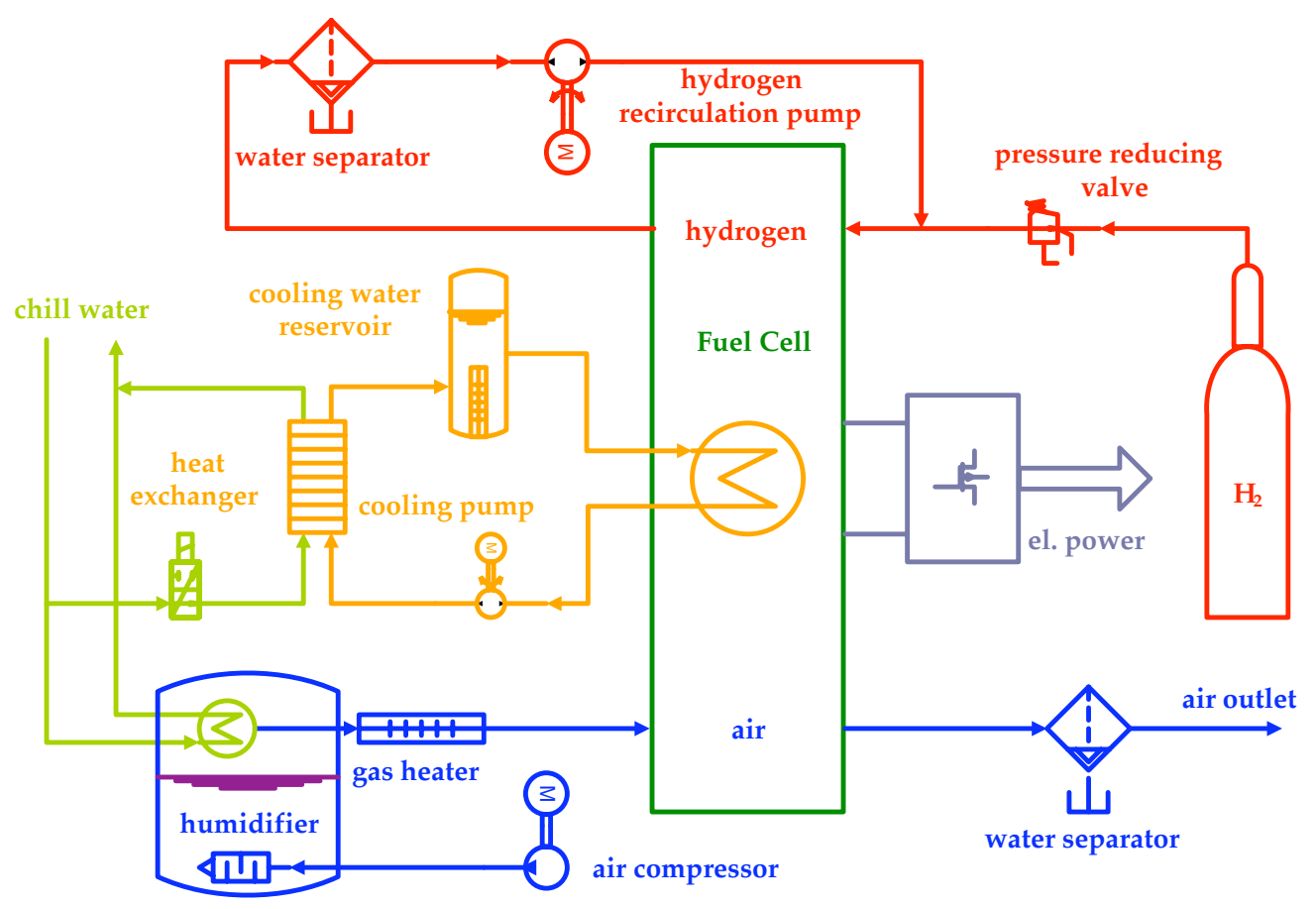

Figure 3.1: Polymer Electrolyte Fuel Cell System.

the oxygen is consumed by the electrochemical reaction, it exits the FC through a water separator into ambient air.

On the hydrogen side, the fuel is introduced into the anode manifold and from there into the FC through a pressure regulator. Upon exiting the FC stack, the leftover hydrogen is re-injected into the anode manifold by a volumetric hydrogen pump. The generated electricity is either fed into a given load or injected into the grid through an adapted power converter. Heat is rejected through a water cooling circuit connected to a liquid/liquid heat exchanger. Cooling water flow is provided by a small, speed controllable water pump.

In a given fuel cell system, not all of these elements are necessarily going to be part of the system. Costs and complexity will limit the number of auxiliary system to a strict minimum necessary to get good system performance. 


\subsection{Air Supply Subsystem Model}

The air supply subsystem consisting of an air compressor, a humidification system, the fuel cell cathode compartment along with the water separator at the exit is shown in Figure 3.1. From a controls point of view, the user input to the air supply subsystem is the voltage applied to the compressor motor. The resulting outputs are an air flow rate and pressure in the FC cathode compartment.

For the air supply dynamics, the humidifier only contributes to the inlet volume and the influence of the water separator at the exit can be neglected. The volume of the FC cathode gas distribution channels is very small compared to the inlet chamber volume (pipes and humidifier) and can therefore be modelled as a simple airflow resistance. The schematic resulting from these assumptions is shown in Figure 3.2.

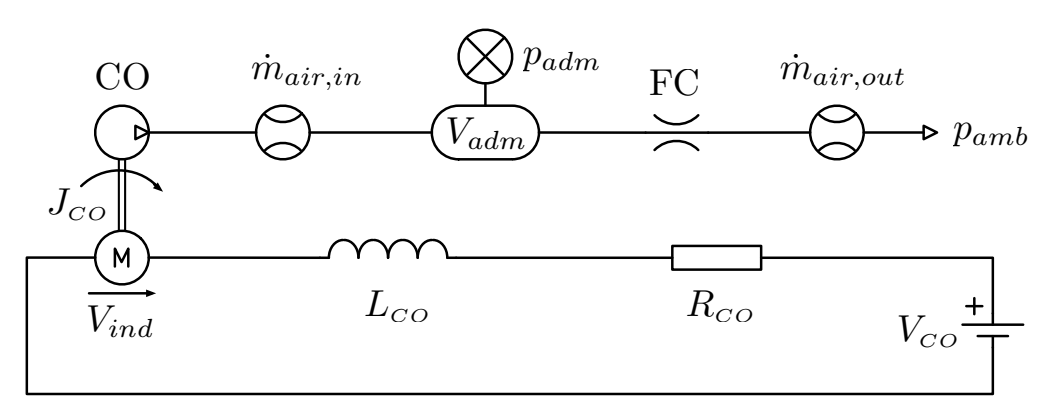

Figure 3.2: Model schematic for the air supply subsystem.

Within such a representation, three different time constants in the system can be distinguished - an electrical time constant related to the current in the compressor, a mechanical time constant related to the inertia of the compressor and a hydraulic time constant related to the volume in the air supply system.

Because of the experimental nature of the implemented humidification system, the humidifier is not included in the model. Inlet air humidity is accounted for through the measurement of the air dew point in the inlet manifold. 


\subsubsection{Air Compressor}

The compressor part is shown more in detail in Figure 3.3. A loop equation on the electrical circuit then yields an expression for the current dynamics.

$$
V_{C O}=L_{C O} \frac{d i_{C O}}{d t}+R_{C O} \cdot i_{C O}+k_{t, C O} \cdot \omega_{C O}
$$

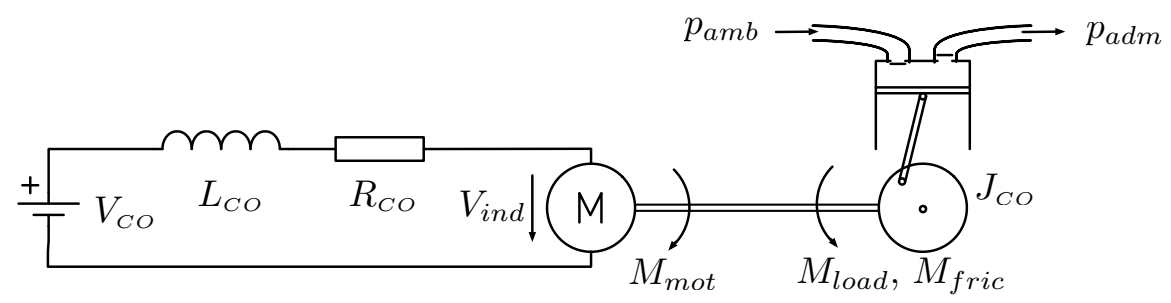

Figure 3.3: Schematic of the air compressor.

For the mechanical side, the speed dynamics are described by a torque balance on the compressor shaft.

$$
J_{C O} \cdot \dot{\omega}_{C O}=M_{m o t}-M_{f r i c}-M_{l o a d}
$$

The load torque being proportional to the difference between the inlet and outlet pressure $\left(p_{a m b}\right.$ and $\left.p_{a i r}\right)$ respectively, (3.2) can be rewritten as

$$
J_{C O} \cdot \dot{\omega}_{C O}=k_{t, C O} \cdot i_{C O}-k_{f, C O} \cdot \omega_{C O}-k_{p, C O}\left(p_{a i r}-p_{a m b}\right)
$$

where the first term on the right represents the motor torque $M_{m o t}$, the second term the friction torque $M_{\text {fric }}$ and the third term the load torque $M_{\text {load }}$.

Measurements on the air compressor have shown a well-approximated linear relationship between compressor speed $\left(\omega_{C O}\right)$ and the resulting air flow rate $\left(\dot{m}_{a i r}\right)$. Assuming a proportionality factor $k_{m, C O},(3.1)$ and (3.3) 
can be written in matrix state space notation (i.e. $\dot{\mathbf{x}}=\mathbf{A} \cdot \mathbf{x}+\mathbf{B} \cdot \mathbf{u}$ and $\mathbf{y}=\mathbf{C} \cdot \mathbf{x}+\mathbf{D} \cdot \mathbf{u})$.

$$
\begin{aligned}
& {\left[\begin{array}{l}
\frac{d i_{C O}}{d t} \\
\frac{d \omega_{C O}}{d t}
\end{array}\right]=\left[\begin{array}{cc}
-\frac{R_{C O}}{L_{C O}} & -\frac{k_{t, C O}}{L_{C O}} \\
\frac{k_{t, C O}}{J_{C O}} & -\frac{k_{f, C O}}{J_{C O}}
\end{array}\right]\left[\begin{array}{c}
i_{C O} \\
\omega_{C O}
\end{array}\right]} \\
& +\left[\begin{array}{ccc}
\frac{1}{L_{C O}} & 0 & 0 \\
0 & -\frac{k_{p, C O}}{J_{C O}} & \frac{k_{p, C O}}{J_{C O}}
\end{array}\right]\left[\begin{array}{c}
V_{C O} \\
p_{a d m} \\
p_{a m b}
\end{array}\right] \\
& {\left[\dot{m}_{a i r, i n}\right]=\left[\begin{array}{ll}
0 & k_{m, C O}
\end{array}\right]\left[\begin{array}{l}
i_{C O} \\
\omega_{C O}
\end{array}\right]}
\end{aligned}
$$

\subsubsection{Pressure Dynamics}

Assuming constant temperature and ideal gas law behaviour, the term describing the pressure dynamics is expressed by (3.6).

$$
\begin{aligned}
\frac{d p_{a d m}}{d t} & =\frac{\mathcal{R} T}{V_{a d m} \mathcal{M}_{a i r}}\left(\dot{m}_{a i r, i n}-\dot{m}_{a i r, \text { out }}\right) \\
& =\frac{\mathcal{R} T}{V_{a d m} \mathcal{M}_{a i r}}\left[\dot{m}_{a i r, i n}-\frac{p_{a d m}-p_{a m b}}{k_{h}}\right]
\end{aligned}
$$

The outlet air flow rate $\dot{m}_{\text {air,out }}$ can be described as a function of the pressure drop across the fuel cell stack. For a laminar flow regime typical of fuel cell applications, it can be shown that the pressure drop is linear with the air flow rate (i.e. $\left.\dot{m}_{a i r, o u t}=\frac{1}{k_{h}}\left(p_{a d m}-p_{a m b}\right)\right)$. Additionally, for a volumetric compressor, the resulting flow rate is proportional to the compressor speed. (i.e. $\dot{m}_{a i r, i n}=k_{m} \cdot \omega_{C O}$ ).

Note that (3.6) can be used to determine the time constant of the pressure evolution within the inlet manifold. With the above assumptions, it can be shown that the time constant is given by:

$$
\tau_{p_{a d m}}=\frac{V_{a d m} \mathcal{M} k_{h}}{\mathcal{R} T} \propto V_{a d m} k_{h}
$$

In order to have a fast air supply system, both the inlet manifold volume $V_{a d m}$ and the hydraulic flow resistance $k_{h}$ of the fuel cell need to be minimised. 


\subsubsection{Overall Air Supply Subsystem Model}

Equations (3.1), (3.3) and (3.6) can then be combined in matrix notation to give a state space description of the air supply subsystem.

$$
\begin{aligned}
& {\left[\begin{array}{c}
\frac{d i_{C O}}{d t} \\
\frac{d \omega_{C O}}{d t} \\
\frac{d p_{a d m}}{d t}
\end{array}\right]=\left[\begin{array}{ccc}
-\frac{R_{C O}}{L_{C O}} & -\frac{k_{t, C O}}{k_{C O}} & 0 \\
\frac{k_{t, C O}}{J_{C O}} & -\frac{k_{f, C O}}{J_{C O}} & -\frac{k_{p, C O}}{J_{C O}} \\
0 & \frac{\mathcal{R} T k_{m, C O}}{V_{a d m} \mathcal{M}_{a i r}} & -\frac{\mathcal{R}^{\prime}}{V_{a d m} \mathcal{M}_{a i r} k_{h}}
\end{array}\right]\left[\begin{array}{c}
i_{C O} \\
\omega_{C O} \\
p_{a d m}
\end{array}\right] } \\
&+ {\left[\begin{array}{cc}
\frac{1}{L_{C O}} & 0 \\
0 & \frac{k_{p, C O}}{J_{C O}} \\
0 & \frac{\mathcal{R} T}{V_{a d m} \mathcal{M}_{a i r} k_{h}}
\end{array}\right]\left[\begin{array}{c}
V_{C O} \\
p_{a m b}
\end{array}\right] } \\
& {\left[\begin{array}{c}
\dot{m}_{\text {air, in }} \\
\dot{m}_{\text {air,out }} \\
p_{\text {adm }}
\end{array}\right]=\left[\begin{array}{ccc}
0 & k_{m} & 0 \\
0 & 0 & \frac{1}{k_{h}} \\
0 & 0 & 1
\end{array}\right]\left[\begin{array}{c}
i_{C O} \\
\omega_{C O} \\
p_{a d m}
\end{array}\right]+\left[\begin{array}{cc}
0 & 0 \\
0 & -\frac{1}{k_{h}} \\
0 & 0
\end{array}\right]\left[\begin{array}{c}
V_{C O} \\
p_{a m b}
\end{array}\right] }
\end{aligned}
$$

In the context of the fuel cell stack model presented in chapter 2, the air flow rate out of the inlet manifold corresponds to the air flow rate through the fuel cell stack (denoted $\dot{m}_{a i r}$ in chapter 2) and the inlet manifold pressure $p_{a d m}$ represents the air inlet pressure of the FC stack (denoted $p_{\text {air }}$ in chapter 2). Additionally, the air flow rate into the inlet manifold does not influence the fuel cell stack directly. Accounting for these notation changes, the output equation relevant to the fuel cell stack model can be rewritten as:

$$
\left[\begin{array}{c}
\dot{m}_{a i r} \\
p_{a i r}
\end{array}\right]=\left[\begin{array}{ccc}
0 & 0 & \frac{1}{k_{h}} \\
0 & 0 & 1
\end{array}\right]\left[\begin{array}{c}
i_{C O} \\
\omega_{C O} \\
p_{a d m}
\end{array}\right]+\left[\begin{array}{cc}
0 & -\frac{1}{k_{h}} \\
0 & 0
\end{array}\right]\left[\begin{array}{c}
V_{C O} \\
p_{a m b}
\end{array}\right]
$$

\subsubsection{Humidifier}

As shown in Figure 3.1, a humidifier controls the air humidity before the cathode inlet. The humidification system installed in the current test bench 
is a lab setup that achieves air humidification through a bubbler system ${ }^{1}$. Due to its energy consumption, such a system would never be part of a stand-alone FC setup where less energy intensive techniques such as water injection into the compressor [50] or stack internal humidification [51] are typically used. Therefore, the humidification system has not been modelled here - humidity is accounted for through direct measurements.

More details on the installed humidification and humidity measurement systems can be found in chapter 4.3.1.

\subsubsection{Backpressure Regulator}

In many fuel cell applications, the fuel cell stack is designed to run at above ambient pressures to increase its performance and efficiency ${ }^{2}$. In such a case, a backpressure regulating valve is fitted at the fuel cell outlet.

Control system oriented modelling of such configurations have been shown by [27] and [29]. This section aims to give further details about the modelling of a backpressure regulator - it includes some experimental results and shows why further work is needed to correctly describe the effect of a user applied valve voltage or current on the resulting air pressure.

The backpressure regulator is basically a variable cross section valve. The cross section can be adjusted by applying a certain coil current that induces a magnetic force acting against a mechanical spring. Increasing coil current increases the magnetic force and results in an increasing cross section of the valve. For a given flow rate that is imposed by the air compressor, the variable valve cross section $A_{c}$ determines the resulting backpressure.

Assimilating the valve to the equivalent circuit shown in Figure 3.4 and assuming limited plunger travel so that the maximum resulting cross section is smaller than the nozzle cross section, the cross section can be expressed as:

$$
A_{c, B R}=\pi \cdot d_{B R} \cdot x_{B R}
$$

The manufacturer's specification indicates a proportional relationship ${ }^{3}$ between the applied valve voltage and the resulting opening of the valve.

\footnotetext{
${ }^{1}$ A heated water bath through which air is "bubbled" to acquire a dew point corresponding to the bath's temperature.

${ }^{2}$ This refers only to the FC stack efficiency - since more compressing power is needed, the overall efficiency can actually decrease.

${ }^{3}$ This can also be obtained by a force balance applied to the valve plunger.
} 


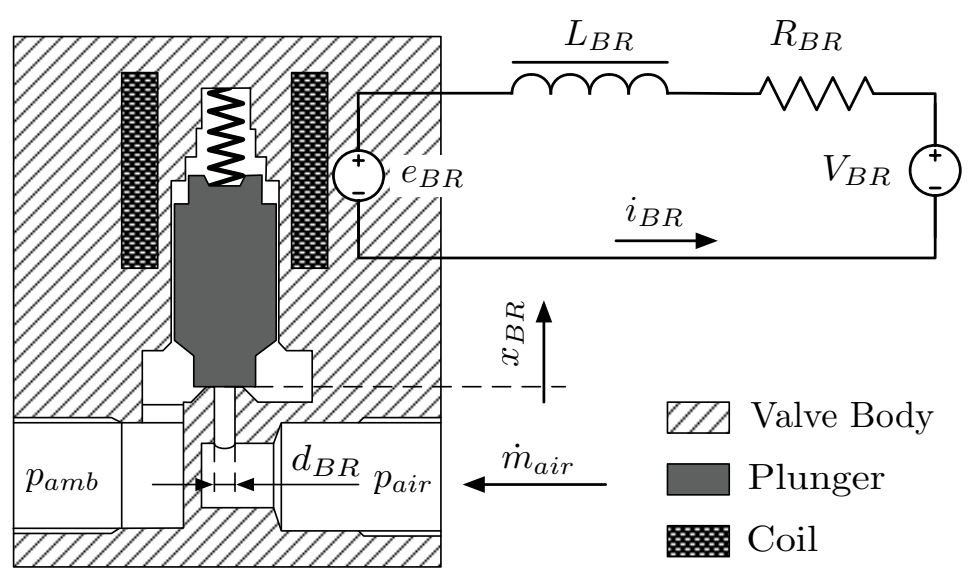

Figure 3.4: Schematic of the backpressure regulator.

The electromechanical time constants for such a valve are typically in the order of 30-40 $\mathrm{ms}$.

The nozzle equation [52] then relates the flow rate for a given cross section to the resulting pressure difference across the valve. Up to a certain critical pressure ratio, the pressure difference will increase exponentially with the air flow rate for steady state conditions.

Experiments have shown (see Figure 3.5) that this description is only representative of large valve openings. When the valve opening is small (i.e. large pressure differences), the valve characteristic seems inverted and assumes a logarithmic behaviour. It is believed that at such small openings, aerodynamic forces acting on the plunger modify its axial position and therefore the valve opening and air pressure.

In order to model the effect of a current or voltage change on the resulting air pressure, a detailed analysis of the backpressure regulator will be required. It needs to formally represent the observed inversion of the valve characteristics at small valve openings.

\subsubsection{Summary}

This section has introduced a linear, state-space model for the dynamics of a non-pressurised air supply system fed by a volumetric compressor. The model describes the evolution of the air flow rate into and out of the inlet

\footnotetext{
${ }^{4}$ Through the manufacturer's electronic control unit, an applied voltage is inversely proportional to the applied valve current $i_{F C}$.
} 


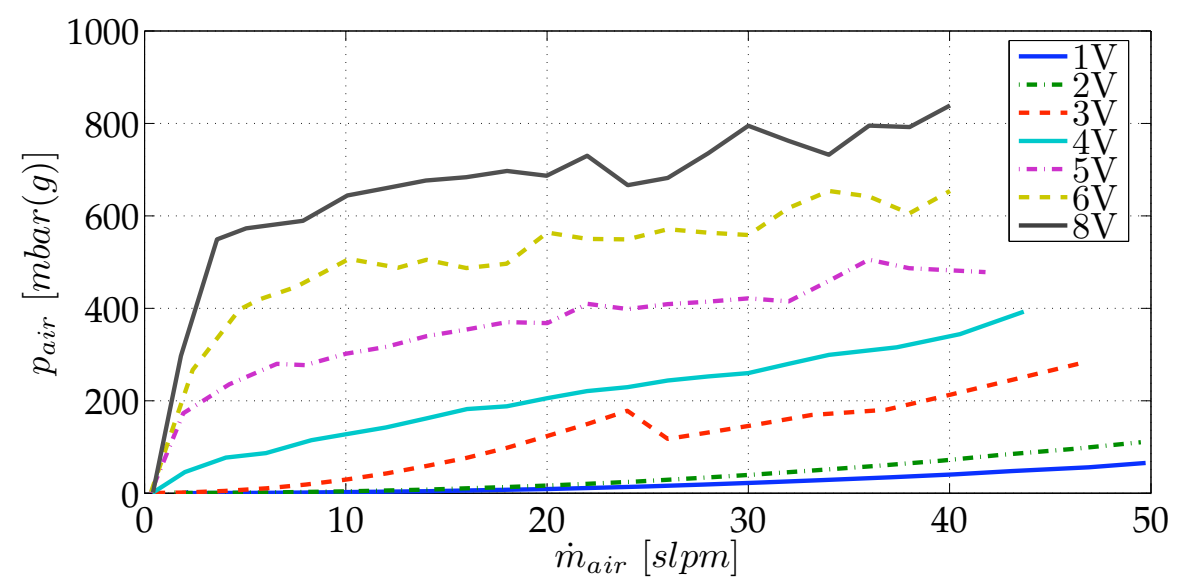

Figure 3.5: Experimental backpressure regulation valve characteristics. The lines for a valve voltage ${ }^{4}$ of 1,2 and (partially) $3 V$ (large valve openings) correspond to the model - at smaller valve openings, the valve characteristics are inverted.

manifold along with the pressure in the inlet manifold. The user supplied compressor voltage along with the ambient pressure are regarded as model inputs.

Problems with the current modelling approaches of a backpressure regulator are outlined and suggestions for future modelling activities in that domain are given.

\subsection{Hydrogen Supply Subsystem Model}

The hydrogen supply subsystem is composed of a pressure regulator connecting it to the hydrogen source, a hydrogen recirculation pump and the FC anode compartment (see Figure 3.1). From a controls point of view, the user input to the hydrogen supply subsystem is the voltage applied to the pump - the resulting output is a hydrogen flow rate through the FC anode compartment.

A hydrogen supply subsystem model can therefore be built based on the schematic shown in Figure 3.6.

The hydrogen pressure within the supply manifold is directly controlled by the pressure regulator. Therefore, the dynamics of the pressure variation 


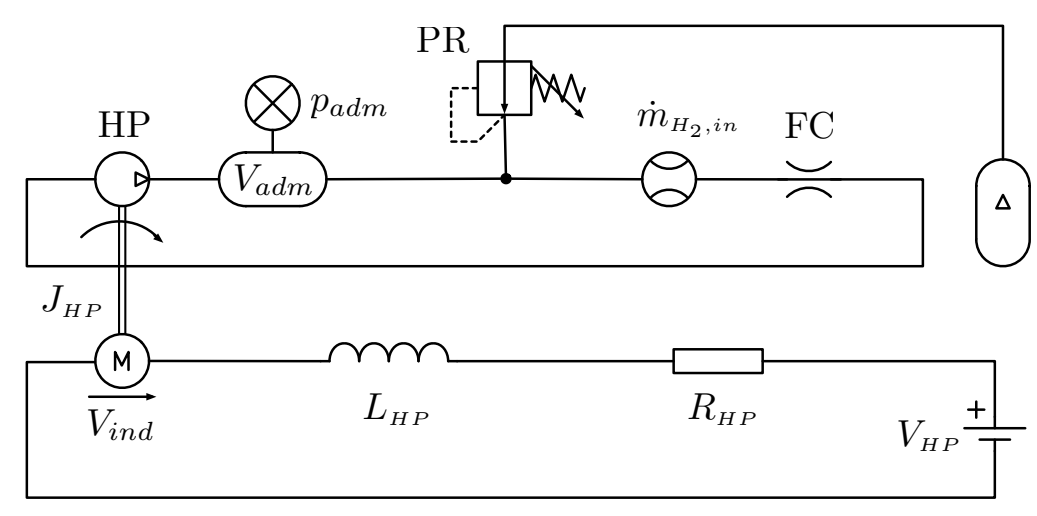

Figure 3.6: Model schematic for the hydrogen supply subsystem.

in the hydrogen compartment then depend on the characteristics of the pressure regulator.

The amount of water within the closed hydrogen supply subsystem can be easily obtained from the water transfer coefficient determined in the fuel cell stack model. However, the molar water fraction at the fuel cell inlet depends on the amount of water that condensates in the system. This has proven very hard to model and hydrogen humidity is therefore accounted for through direct measurement.

\subsubsection{Hydrogen Recirculation Pump}

Equations (3.1) and (3.3) can be adapted to the hydrogen recirculation pump resulting in the state space model shown in (3.12) and (3.13). Note that, because of the assumption of laminar flow conditions, the pressure drop term depends linearly on the flow rate and thus the pump speed. Its contribution has therefore been integrated into an overall friction coefficient $k_{f, H P}$.

$$
\begin{aligned}
{\left[\begin{array}{c}
\frac{d i_{H P}}{d t} \\
\frac{d \omega_{H P}}{d t}
\end{array}\right]=} & {\left[\begin{array}{rr}
-\frac{R_{H P}}{L_{H P}} & -\frac{k_{t, H P}}{L_{H P}} \\
\frac{k_{t, H P}}{J_{H P}} & -\frac{k_{f, H P}}{J_{H P}}
\end{array}\right]\left[\begin{array}{c}
i_{H P} \\
\omega_{H P}
\end{array}\right] } \\
& +\left[\begin{array}{c}
\frac{1}{L_{H P}} \\
0
\end{array}\right]\left[V_{H P}\right]
\end{aligned}
$$




$$
\left[\dot{m}_{H_{2}}\right]=\left[\begin{array}{ll}
0 & k_{m, H P}
\end{array}\right]\left[\begin{array}{c}
i_{H P} \\
\omega_{H P}
\end{array}\right]
$$

For the test stand installed at $L E I$, the motor amplifier running the hydrogen recirculation pump already has a fast current controller built in. In this case, the motor current set-value becomes the user input and (3.12) reduces to

$$
\begin{gathered}
{\left[\frac{d \omega_{H P}}{d t}\right]=\left[-\frac{k_{f, H P}}{J_{H P}}\right]\left[\omega_{H P}\right]+\left[\frac{k_{t, H P}}{J_{H P}}\right]\left[i_{H P}\right]} \\
{\left[\dot{m}_{H_{2}}\right]=\left[k_{m, H P}\right]\left[\omega_{H P}\right]}
\end{gathered}
$$

\subsubsection{Hydrogen Pressure Regulator}

The pressure in the hydrogen compartment is controlled by a mechanical pressure regulator (see Figure 3.6). The pressure regulator interfaces a high pressure (typically between 50 to 250 bar) hydrogen storage volume to the low pressure hydrogen supply manifold. By pre-loading a spring, the user sets a desired operating pressure. During operation, a control valve within the pressure regulator adjusts the flow rate into the hydrogen compartment to control the pressure to the desired level.

Through a number of interconnected sensing volumes, a hydro-mechanical feedback loop is created to regulate the pressure to the level pre-set through the pre-loaded spring. The dynamics of the closed loop regulation depend on the exact geometry of the different sensing volumes and interconnections along with the friction and stiction characteristics.

A first steady state pressure regulator model was proposed by Iberall et al. [53]. Tsai et al. [54] later developed a dynamic model of a spring loaded pressure regulator. Since their models do not take any stiction into account, they are not able to predict the experimentally observed behaviour of the pressure regulator installed on the test bench (see Figure 3.8). 


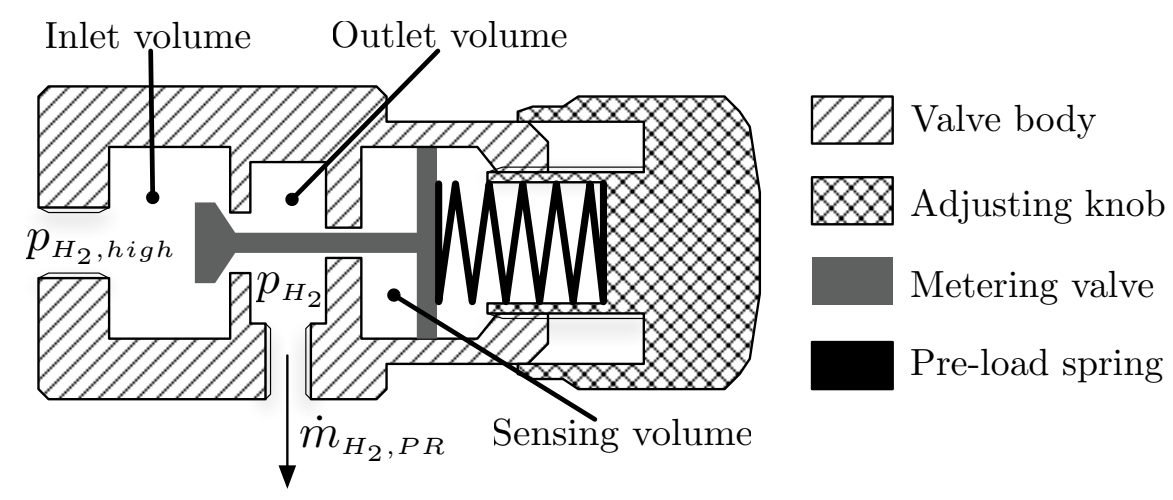

Figure 3.7: Schematic drawing of a spring loaded pressure regulator with one sensing volume.

\subsubsection{Hydrogen Pressure Dynamics}

Since the hydrogen supply (HS) manifold represents a closed circuit, the pressure dynamics can be expressed through a mass balance on the manifold volume. This can be written as:

$$
\begin{aligned}
\frac{d p_{H S}}{d t} & =\frac{\mathcal{R} T}{V_{H S} \mathcal{M}_{H_{2}}}\left(\dot{m}_{H_{2}, \text { in }}-\dot{m}_{H_{2}, \text { out }}\right) \\
& =\frac{\mathcal{R} T}{V_{H S} \mathcal{M}_{H_{2}}}\left[\dot{m}_{H_{2}, P R}-\frac{I_{e l}}{2 F} \mathcal{M}_{H_{2}}\right]
\end{aligned}
$$

where $\dot{m}_{H_{2}, P R}=f\left(p_{H S}\right)$ is a function of the hydrogen pressure. Since this relationship is difficult to model $^{5}$, the pressure dynamics are not described through a model in the context of this thesis.

The hydrogen pressure dynamics have been verified experimentally by suddenly opening a discharge valve with a certain flow resistance on the hydrogen supply manifold, pressurised at 500 mbar(a). The resulting pressure evolution is plotted for the case with and without pressure regulator on Figure 3.8.

\footnotetext{
${ }^{5}$ See chapter 3.4.2.
} 


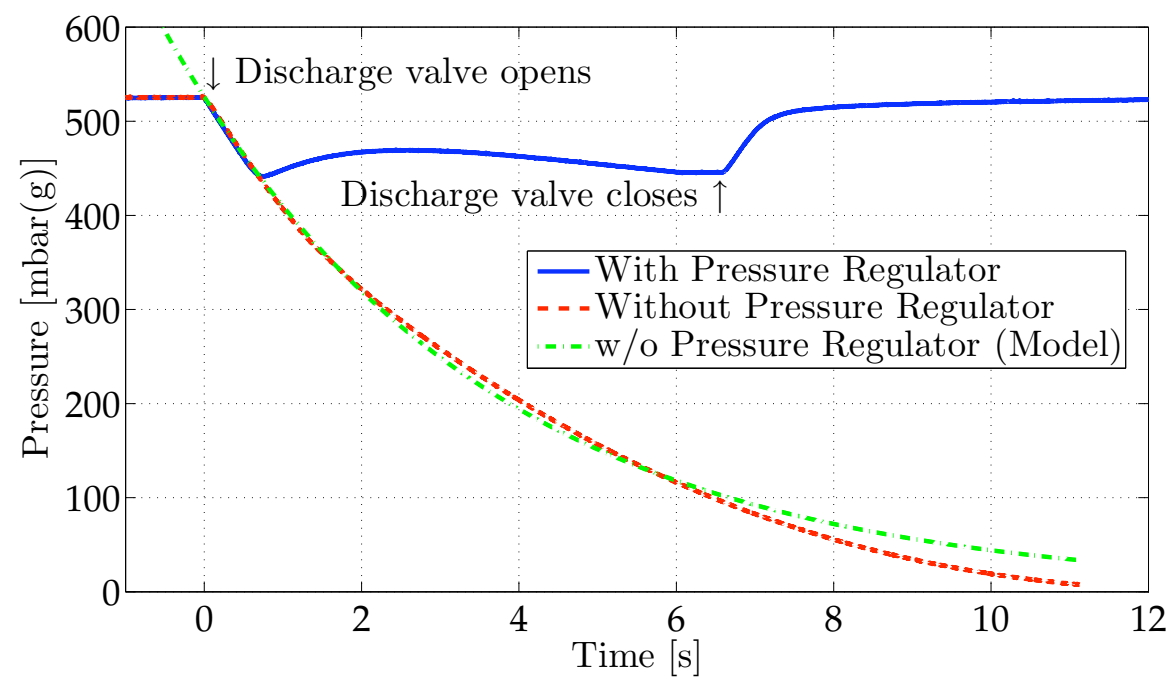

Figure 3.8: Pressure evolution in the hydrogen supply manifold following a sudden opening of a discharge valve. The results are shown with and without pressure regulator.

If a discharge flow rate $\dot{m}_{H_{2}, \text { out }}=k_{D V}\left(p_{H S}-p_{a m b}\right)$ is assumed, the pressure variation can be shown to follow an exponential law with a time constant

$$
\tau_{H S}=\frac{V_{H S}}{k_{D V} \mathcal{R} T}
$$

With the discharge coefficient $k_{D V}$ and the volume of the hydrogen supply manifold, this results in a time constant of $\tau_{H S} \approx 4 s$ which is verified experimentally when no pressure regulator is present. With the pressure regulator, a difference of around 80 mbar between the setpoint and the actual pressure is required before the regulator starts injecting hydrogen. During this time, the pressure evolution is governed by the time constant established in (3.17). Under steady state conditions with the discharge valve open, a permanent error of around 80 mbar subsists.

\subsubsection{Summary}

This section has introduced a linear, state-space model for the dynamics of the hydrogen flow rate in the fuel cell. The model considers a closed 
loop hydrogen supply where the flow rate is imposed through a volumetric pump.

The hydrogen pressure in the manifold is imposed by a mechanical pressure regulator. The problems with current pressure regulator models are introduced and a comparison between theoretical and actual hydrogen pressure dynamics is shown.

\subsection{Thermal Management Subsystem Model}

The thermal management subsystem is shown in detail below (Figure 3.9). It is composed of the cooling channels integrated into the fuel cell's bipolar plates, a variable speed cooling liquid (CL) pump (CP) and a liquid-liquid heat exchanger (HX) to reject any surplus heat to a chill water circuit (CW) (see Figure 3.1). The experimental setup also integrates a cooling water reservoir (RV) with an electrical heating element, allowing the system to be brought to operating temperature prior to switch-on along with maintaining operating temperature in low-load conditions.

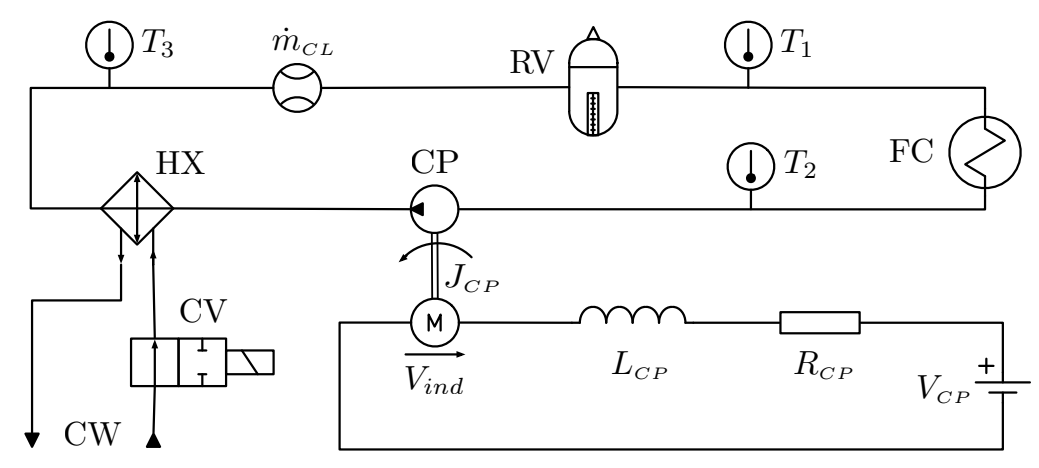

Figure 3.9: Model schematic for the thermal management subsystem.

This chapter will establish a state-space dynamic model describing the temperature evolution of the fuel cell and cooling liquid reservoir as a function of the user inputs and ambient conditions. The losses in the fuel cell $\dot{Q}_{l o s s}$, the heating power in the cooling liquid reservoir $\dot{Q}_{\text {heat }}$, the voltage applied to the cooling liquid pump $V_{C P}$ and the duty cycle applied to the chill water valve $d$ are considered as user inputs. Ambient conditions are represented by the ambient temperature $T_{a m b}$ and the chill water temperature $T_{C W}$. 
In order to describe the main dynamics of the system, the heat transfer processes occurring in the components of the thermal management subsystem are described for each component in chapters 3.5.1 to 3.5.5. In this context, the FC and the water reservoir are considered as lumped masses with associated dynamics while the heat exchanger is considered as a heat transfer element only. The cooling liquid temperature is assumed constant within the connecting hoses.

Sections 3.5.6 to 3.5.8 then assemble the presented governing equations for each component and derive a non-linear state space model that is then linearised around a given operating point to be presented in matrix notation.

\subsubsection{Cooling Pump}

The cooling pump's $(\mathrm{CP})$ role in the thermal management subsystem is to provide a cooling liquid flow at a desired flow rate. The user adjusts the pump speed by applying a voltage $V_{H P}$ to the pump motor. The resulting cooling liquid flow rate can then be considered an input to the other elements in the cooling circuit - the higher the flow rate, the more heat can be transferred from one element to the next.

In order to describe the dynamics of the cooling pump, it can be treated like the air compressor shown in chapter 3.3.1. The primary difference resides in the fact that the $\mathrm{CP}$ inertia and friction coefficient not only comprises the mechanical inertia and friction, but also the inertia of the cooling liquid (CL) and its friction on the tube walls ${ }^{6}$. This can be expressed as:

$$
\begin{gathered}
{\left[\begin{array}{c}
\frac{d i_{C P}}{d t} \\
\frac{d \omega_{C P}}{d t}
\end{array}\right]=\left[\begin{array}{cc}
-\frac{R_{C P}}{L_{C P}} & -\frac{k_{t, C P}}{L_{C P}} \\
\frac{k_{t, C P}}{J_{C P}} & -\frac{k_{f, C P}}{J_{C P}}
\end{array}\right]\left[\begin{array}{c}
i_{C P} \\
\omega_{C P}
\end{array}\right]+\left[\begin{array}{c}
1 \\
L_{C P} \\
0
\end{array}\right]\left[V_{C P}\right]} \\
{\left[\dot{m}_{C L}\right]=\left[\begin{array}{ll}
0 & k_{m, C P}
\end{array}\right]\left[\begin{array}{c}
i_{C P} \\
\omega_{C P}
\end{array}\right]}
\end{gathered}
$$

\footnotetext{
${ }^{6}$ Assuming laminar flow in the cooling circuit and neglecting minor losses, it can be shown that the pressure drop in the circuit is proportional to the flow rate and therefore to the compressor speed. As shown in chapter 3.3.1, a pressure difference across a volumetric compressor is proportional to the compressor load torque. Therefore, those hydrodynamic friction terms can be directly included in a global viscous friction term for the cooling pump.
} 
It can be shown that the electrical time constant $\left(\tau_{e l} \approx 0.005 \mathrm{~s}\right)$ and the mechanical time constant $\left(\tau_{m e c} \approx 0.5 s\right.$ ) of the cooling pump are significantly smaller than the dominant thermal time constants of the cooling circuit.

Therefore, the state space model of the cooling pump can be reduced to its steady state input-output description. Setting all time derivatives in (3.18) and (3.19) to zero and solving for the CL mass flow rate yields:

$$
\dot{m}_{C L}=\frac{k_{t, C P} \frac{V_{C P}}{R_{C P}}}{k_{m, C P}\left(k_{f, C P}+\frac{k_{t, C P}{ }^{2}}{R_{C P}}\right)}=k_{C P} V_{C P}
$$

Since all terms in (3.20) apart from the pump voltage $V_{C P}$ are characteristic for the pump in question, they can be replaced with a single, experimentally identifiable constant $k_{C P}$ as shown.

The steady state pump speed and current are given $b^{7}$ :

$$
\begin{gathered}
i_{C P}=\frac{V_{C P}}{R_{C P}+\frac{k_{t, C P}^{2}}{k_{f, C P}}} \\
\omega_{C P}=\frac{V_{C P}}{\frac{R_{C P} k_{f, C P}}{k_{t, C P}}-k_{t, C P}}
\end{gathered}
$$

The power consumption of the cooling pump can then be easily determined by multiplying (3.21) by the pump voltage. This yields:

$$
P_{C P}=\frac{V_{C P}^{2}}{R_{C P}+\frac{k_{t, C P}^{2}}{k_{f, C P}}}
$$

\subsubsection{Thermal Fuel Cell Model}

In the fuel cell, the losses described in chapter 2.3 lead to a heat production. The heat is dissipated in the regions where the respective losses occur and is then evacuated from the cell through conduction to the bipolar plates and from there through convection to the ambient air and cooling liquid.

\footnotetext{
${ }^{7}$ This is obtained by setting all time derivatives in (3.18) to zero.
} 
This leads to temperature gradients in the direction perpendicular to the cell. However, they are generally negligible with respect to nonuniform in-plane temperature distribution (hot spots) as has been shown by Freunberger et al. [55]. Therefore, the fuel cell will be considered isothermal in the context of this thesis.

Furthermore, hydrogen and air are assumed to be supplied to the fuel cell at cell temperature and thus do not contribute to the temperature evolution of the system. Any liquid that condenses or evaporates within the system will contribute to the temperature evolution. However, since the fuel cell stack model presented in chapter 2 does not describe the condensation or evaporation of water, this contribution cannot be accounted for in the thermal model.

It can be shown that for the considered stack [56] and cooling pump, the Biot number in the cooling channels is inferior to $B i \leq 0.2$. This signifies that the the conduction phenomena within the plate presents much less heat transfer resistance than the convective term. Therefore, the stack can be treated as a lumped capacitance model [57] and a constant bipolar plate temperature can be assumed.

Applying a basic energy balance for the bipolar plate allows to express the evolution of the fuel cell temperature as

$$
\begin{aligned}
\frac{d T_{F C}}{d t}= & \frac{1}{m_{F C} c_{p, F C}}\left[\dot{Q}_{l o s s}+\dot{m}_{C L} \cdot c_{p, C L}\left(T_{1}-T_{2}\right)\right. \\
& \left.-k_{F C}\left(T_{F C}-T_{a m b}\right)\right]
\end{aligned}
$$

where the following phenomena have been taken into account:

Natural convection with the ambient air can be shown to closely follow a law of the form

$$
\dot{Q}_{c o n v}=-k_{F C}\left(T_{F C}-T_{a m b}\right)
$$

as shown on Figure 3.10. The logarithm of the Raleigh number $\left(R a_{L}\right)$ being inferior to 9 indicates a laminar flow regime [57]. If the fuel cell is considered as a flat plate, an average value for the natural convection coefficient of $k_{F C} \approx 0.7 \mathrm{~W} / \mathrm{K}$ results.

Forced internal convection in the cooling channels is described through an energy balance between inlet and outlet temperatures. 


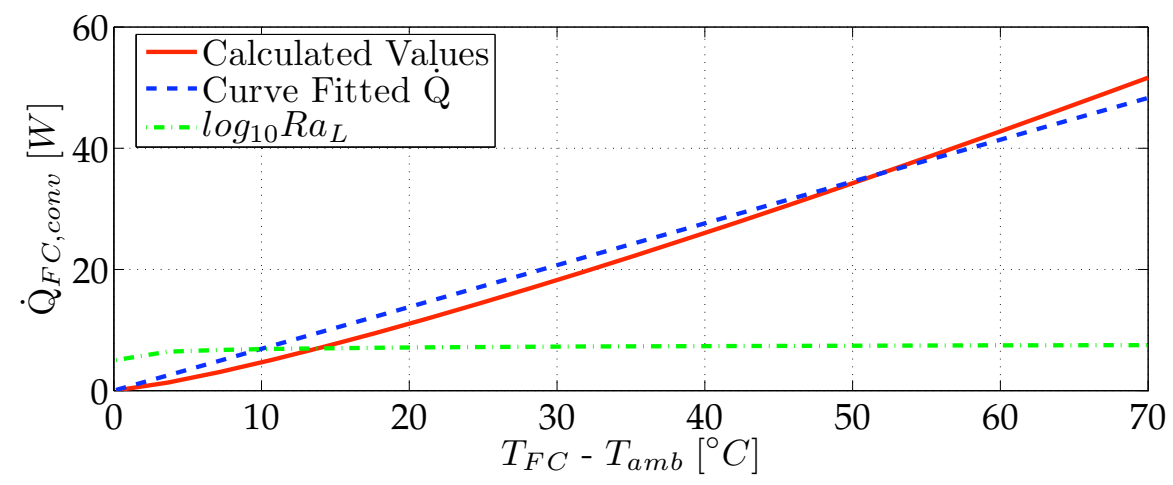

Figure 3.10: Heat transfer through free convection along the fuel cell that is considered as a vertical flat plate.

Fuel Cell Losses are included as a heat source term. According to the definition of the fuel cell losses in chapter 2.3, the dissipated heat per cell can be written as:

$$
\dot{Q}_{\text {loss }}=\left(E_{0}-\bar{E}\right) I_{e l}
$$

Since the air and hydrogen supplied to the fuel cell enter the cell at a temperature close to the one of the fuel cell, convective cooling through process gases has not been accounted for. Also, because the fuel cell model does not distinguish between gaseous and liquid water, the effects of water vaporisation and condensation on the cell temperature have been neglected.

Note that since $T_{1}$ is a measure of the CL temperature at the reservoir exit, $T_{1}=T_{R V}$ can be assumed. The FC exit temperature $T_{2}$ is determined by the heat transferred to the CL along the cooling channels. Describing the heat transfer through forced internal convection, this can be expressed as:

$$
T_{2}=T_{F C}-\left(T_{F C}-T_{R V}\right) \exp \left(-\frac{A_{s} \cdot h_{F C}}{\dot{m}_{C L} \cdot c_{p, C L}}\right)
$$

The equivalent average convection coefficient $h_{F C}$ can be determined through experimental Nusselt number correlations [58].

\subsubsection{Cooling Liquid Reservoir}

The cooling liquid reservoir serves as a storage volume for the cooling liquid and permits evacuation of any air in the cooling circuit. In order to bring 
the fuel cell up to operating temperature prior to start-up, an electrical heating cartridge has been integrated into the reservoir. The heat supplied by the heater can be adjusted by the user and is denoted as $\dot{Q}_{\text {heat }}$.

Because the reservoir and the cooling liquid it contains represent a large thermal inertia, a time dependent formulation for the temperature evolution has to be found. In analogy with (3.24), the cooling liquid temperature in the reservoir is expressed as:

$$
\begin{aligned}
\frac{d T_{R V}}{d t}= & \frac{1}{m_{R V} c_{p, R V}}\left[\dot{Q}_{\text {heat }}+\dot{m}_{C L} \cdot c_{p, C L}\left(T_{3}-T_{R V}\right)\right. \\
& \left.-k_{R V}\left(T_{R V}-T_{a m b}\right)\right]
\end{aligned}
$$

In this context, the natural convection heat transfer coefficient $k_{R V}$ comprises the natural convection within the reservoir, within the heat exchanger and the cooling liquid tubing.

\subsubsection{Liquid-Liquid Heat Exchanger}

The liquid-liquid type heat exchanger is used to reject waste heat from the fuel cell. The cooling liquid heated in the fuel cell transfers heat to a chill water circuit as it passes through the heat exchanger. In order to obtain the CL temperature at the HX outlet, the heat transfer within the HX needs to be described as a function of the chill water and cooling liquid flow rates.

This is achieved by using an NTU analogy [57]. The number of transfer units (NTU) is characteristic for a given heat exchanger and is defined as:

$$
N T U=\frac{U A}{C_{\min }}
$$

where:

$$
\begin{array}{rlr}
A & =\text { Heat exchange surface area } & {\left[m^{2}\right]} \\
U & =\text { Overall heat transfer coefficient } & {\left[\frac{W}{m^{2} K}\right]} \\
C_{\text {min }} & =\min \left[c_{p, h} \cdot \dot{m}_{h}, c_{p, c} \cdot \dot{m}_{c}\right] & {\left[\frac{k J}{K}\right]}
\end{array}
$$


Based on a known NTU value ${ }^{8}$, a heat exchanger efficiency $\epsilon$ can be calculated according to the following equations:

$$
\begin{gathered}
\epsilon=1-e^{\frac{1}{C_{R}} N T U^{0.22}\left[e^{\left(-C_{R} N T U^{0.78}\right)}-1\right]} \\
\epsilon=\frac{1-e^{-N T U\left(1-C_{R}\right)}}{1-C_{R} e^{-N T U\left(1-C_{R}\right)}} \quad \text { for } C_{R}<1 \\
\epsilon=\frac{N T U}{1+N T U} \quad \text { for } C_{R}=1
\end{gathered}
$$

$$
\text { where: } \begin{aligned}
C_{R} & =\frac{C_{\min }}{C_{\max }} \\
C_{\max } & =\max \left[c_{p, h} \cdot \dot{m}_{h}, c_{p, c} \cdot \dot{m}_{c}\right]
\end{aligned}
$$

(3.30) is applicable for a cross flow type heat exchanger and (3.31) and (3.32) are applicable for counter flow type heat exchangers.
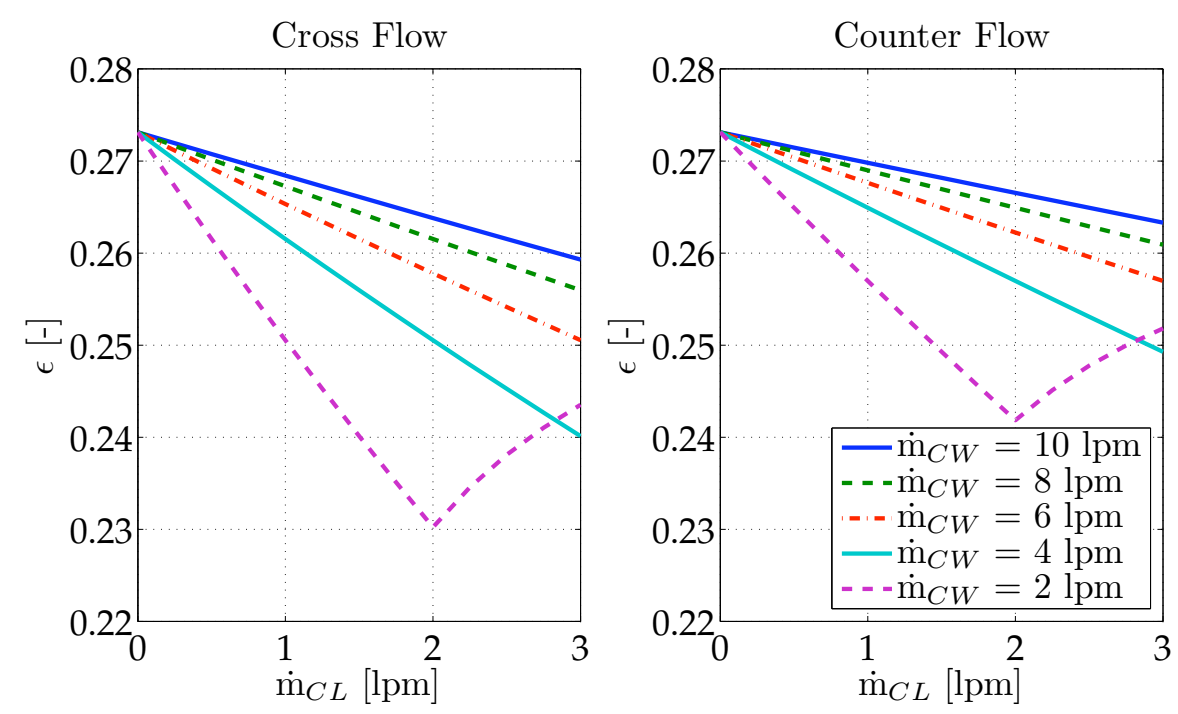

Figure 3.11: Heat exchanger efficiency $\epsilon$ for various hot and cold water flow rate conditions.

\footnotetext{
${ }^{8}$ The NTU value of a heat exchanger can be either calculated based on experimental data or obtained from the manufacturer.
} 
As shown in Figure 3.11, for as long as $C_{C L}<C_{C W}$, (3.30) and (3.31) result in a linear relationship for $\epsilon$ of the form

$$
\epsilon=\epsilon_{0}-k_{x} \dot{m}_{C L}
$$

where $k_{x}$ is the heat exchanger efficiency-flow rate coefficient for a given HX. $\epsilon_{0}$ is a constant independent of the HX type and is given by (3.31) as $C_{R}$ approaches zero.

$$
\epsilon_{0}=1-e^{-N T U} \approx 0.273
$$

For the configuration of the LEI test stand (see chapter 4), the chill water flow rate $\dot{m}_{C W}=8.5 \mathrm{lpm}$ is at least four times the cooling liquid flow rate. Therefore, the HX efficiency can be expressed using the linear formulation (3.33). Additionally, it can be shown that the relative difference between the efficiency of a cross-flow and a counter-flow type HX is below $2 \%$.

The heat transfer in the HX can then be described for two distinct cases:

1. When the chill water flow through the HX is turned off, i.e. $C_{C W}=0$, no heat transfer occurs and the inlet and outlet temperatures are the same.

$$
T_{3}=T_{2}
$$

2. When the chill water flow through the HX is turned on, i.e. $C_{C W} \neq 0$, and while $C_{C L}<C_{C W}, T_{3}$ can be expressed as:

$$
T_{3}=\epsilon\left(T_{C W}-T_{2}\right)+T_{2}
$$

where $\epsilon$ is the heat exchanger efficiency that depends linearly on the cooling liquid flow.

The overall heat transfer can be expressed as a function of the chill water and cooling liquid inlet temperatures.

$$
\dot{Q}=\epsilon C_{\min }\left(T_{C W}-T_{2}\right)
$$

If the ranges of the $\mathrm{CW}$ and $\mathrm{CL}$ flow rates cross, a discontinuity of the heat transfer will occur along the line where $\dot{m}_{C L}=\dot{m}_{C W}$. If the hardware configuration is such that this is the case, care needs to be taken to implement the change in heat transfer as the CW flow rate is reduced below the CL flow. 


\subsubsection{Liquid-Air Heat Exchanger}

Alternatively, the surplus heat from the fuel cell could be rejected through an air-liquid heat exchanger. Such a system would be typically assisted by a cooling fan forcing ambient air through the radiator.

For such heat exchangers, the heat transfer can be shown to be proportional to the temperature difference between the ambient air and the cooling liquid at the inlet $T_{2}$. The proportionality factor is denoted $k_{H X}$ and depends on the air flow through the HX.

$$
T_{3}=\frac{\left(T_{a m b}-T_{2}\right) k_{H X}}{\dot{m}_{C L} \cdot c_{p, C L}}+T_{2}
$$

If the cooling fan speed can be adjusted continuously, the specific heat exchanger power $k_{H X}$ can be expressed as a function of the fan speed and therefore as a function of the user input applied to the HX.

If the fan can only be switched on or off, the heat transfer is described for each case through the corresponding specific heat exchanger power $k_{H X}^{o n}$ and $k_{H X}^{o f f}$. These descriptions can then be combined into an overall description as described in chapter 3.5.8.

\subsubsection{Overall Cooling Circuit}

(3.24), (3.27), (3.28) along with either (3.36) and (3.35) or (3.38) can then be regrouped to yield an expression for the time derivative of the bipolar plate and cooling liquid reservoir temperatures. Since the system installed at LEI is composed of a liquid-liquid heat exchanger, only that configuration will be shown here.

The fuel cell temperature can be expressed as:

$$
\begin{aligned}
\frac{d T_{F C}}{d t}= & \frac{1}{m_{F C} c_{p, B P}}\left[\dot{Q}_{\text {loss }}-k_{F C}\left(T_{F C}-T_{a m b}\right)+\dot{m}_{C L} c_{p, C L}\right. \\
& {\left.\left[T_{1}-T_{F C}+\left(T_{F C}-T_{R V}\right) \exp \left(-\frac{A_{s} h_{F C}}{\dot{m}_{C L} c_{p, C L}}\right)\right]\right] }
\end{aligned}
$$


And the cooling water reservoir temperature is given by (for the HX turned on and off respectively):

$$
\begin{aligned}
\frac{d T_{R V, \text { on }}}{d t}= & \frac{1}{m_{R V} c_{p, C L}}\left[\dot{Q}_{\text {heat }}-k_{R V}\left(T_{R V}-T_{a m b}\right)+\dot{m}_{C L} c_{p, C L}\right. \\
& {\left[\epsilon_{0}-k_{x} \dot{m}_{C L} T_{C W}+\left(1-\epsilon_{0}+k_{x} \dot{m}_{C L}\right)\right.} \\
& \left.\left.\left(T_{F C}-\left(T_{F C}-T_{R V}\right) \exp \left(-\frac{A_{s} h_{F C}}{\dot{m}_{C L} c_{p, C L}}\right)\right)-T_{R V}\right]\right] \\
\frac{d T_{R V, \text { off }}}{d t}= & \frac{1}{m_{R V} c_{p, C L}}\left[\dot{Q}_{h e a t}-k_{R V}\left(T_{R V}-T_{a m b}\right)+\dot{m}_{C L} c_{p, C L}\right. \\
& {\left.\left[T_{F C}-\left(T_{F C}-T_{R V}\right) \exp \left(-\frac{A_{s} h_{F C}}{\dot{m}_{C L} c_{p, C L}}\right)-T_{R V}\right]\right] }
\end{aligned}
$$

\subsubsection{State Space Representation of the Overall Cooling Circuit}

(3.39) to (3.41) can be viewed as a state space expression of the system with states $T_{F C}$ and $T_{R V}$ and inputs $\dot{Q}_{\text {loss }} \dot{Q}_{\text {heat }} \dot{m}_{C L}$ and $T_{C W}$. Additionally, (3.20) describes the cooling liquid flow rate $\dot{m}_{C L}$ as a function of the user input $V_{C P}$ and can therefore be substituted into (3.39) to (3.41).

Since these governing equations are not linear, they cannot be expressed in matrix notation. However, linearising the system around a given operating point (state space variables and system inputs), allows to express the system's dynamics in a state space matrix form. Denoting the operating point with $\bar{x}$ and the deviation from that operating point as $\tilde{x}$, yields for the HX turned on and off respectively ${ }^{9}$ :

$$
\left[\frac{d \tilde{T}_{F C}}{d t}\right]=\left[\frac{\mathbf{A}_{C C}}{d t}\right]\left[\begin{array}{c}
\tilde{T}_{F C} \\
\tilde{T}_{R V}
\end{array}\right]+\left[\mathbf{B}_{C C}\right]\left[\begin{array}{c}
\tilde{\dot{Q}}_{\text {loss }} \\
\tilde{\dot{Q}}_{\text {heat }} \\
\tilde{V}_{C P} \\
\tilde{T}_{C W} \\
\tilde{T}_{\text {amb }}
\end{array}\right]
$$

\footnotetext{
${ }^{9}$ See Appendix D for more details on the state space notation of a linearised system.
} 


$$
\left[T_{F C}\right]=\left[\begin{array}{ll}
1 & 0
\end{array}\right]\left[\begin{array}{l}
\bar{T}_{F C}+\tilde{T}_{F C} \\
\bar{T}_{R V}+\tilde{T}_{R V}
\end{array}\right]
$$

where $A_{C C}$ and $B_{C C}$ are the Jacobian matrices of (3.39) to (3.41) with respect to the states and system inputs. They are given for the HX turned on as:

$$
\mathbf{A}_{C C, \text { on }}=\left[\begin{array}{cc}
-\frac{F(1-E)+k_{F C}}{G} & \frac{F(1-E)}{G} \\
\mathbf{A}_{C C, \text { on }}(2,1) & \mathbf{A}_{C C, \text { on }}(2,2)
\end{array}\right]
$$

Where $\mathbf{A}_{C C, \text { on }}(2,1)$ and $\mathbf{A}_{C C, \text { on }}(2,2)$ are given by:

$$
\begin{gathered}
\left.\mathbf{A}_{C C, \text { on }}(2,1)\right)=\frac{F(1-E)\left(1-\epsilon_{0}+k_{x} \overline{\dot{m}}_{C L}\right)}{H} \\
\mathbf{A}_{C C, \text { on }}(2,2)=\frac{F\left(E\left(1-\epsilon_{0}+k_{x} \overline{\dot{m}}_{C L}\right)-1\right)-k_{R V}}{H} \\
B_{C C, \text { on }}=\left[\begin{array}{ccccc}
\frac{1}{G} & 0 & B_{C C, \text { on }}(1,3) & 0 & \frac{k_{F C}}{G} \\
0 & \frac{1}{H} & B_{C C, \text { on }}(2,3) & \frac{F}{H}\left(\epsilon_{0}-k_{x} \overline{\dot{m}}_{C L}\right) & \frac{k_{R V}}{H}
\end{array}\right]
\end{gathered}
$$

Where $B_{C C, \text { on }}(1,3)$ and $B_{C C, \text { on }}(2,3)$ are given by:

$$
\begin{aligned}
B_{C C, \text { on }}(1,3)= & \frac{c_{p, C L}\left(\bar{T}_{R V}(1-E)-\bar{T}_{F C}(1-E)\right)}{G} \\
& +\frac{\frac{E\left(\bar{T}_{F C}-\bar{T}_{R V}\right) A_{s} \cdot h_{F C}}{\dot{m}_{C L}}}{G}
\end{aligned}
$$




$$
\begin{aligned}
B_{C C, \text { on }}(2,3)= & \frac{c_{p, C L}}{H}\left[\left(\epsilon_{0}-k_{x} \overline{\dot{m}}_{C L}\right) \bar{T}_{C W}-\bar{T}_{R V}\right. \\
& \left.+\left(\bar{T}_{F C}(1-E)+\bar{T}_{R V} E\right)\left(1-\epsilon_{0}+k_{x} \overline{\dot{m}}_{C L}\right)\right] \\
& +\frac{F}{H}\left[-k_{x} \bar{T}_{C W}+k_{x}\left(\bar{T}_{F C}-E\left(\bar{T}_{F C}-\bar{T}_{R V}\right)\right)\right. \\
& \left.-\frac{E\left(\bar{T}_{F C}-\bar{T}_{R V}\right) A_{s} \cdot h_{F C}\left(1-\epsilon_{0}+k_{x} \overline{\dot{m}}_{C L}\right)}{\dot{m}_{C L} F}\right]
\end{aligned}
$$

where: $\quad E=\exp \left(-\frac{A_{s} h_{F C}}{\overline{\dot{m}}_{C L} c_{p, C L}}\right)$

$$
\begin{aligned}
F & =\overline{\dot{m}}_{C L} \cdot c_{p, C L} \stackrel{m_{C L} c_{p L}}{=} k_{C P} \bar{V}_{C P} \\
G & =m_{F C} \cdot c_{p, B P} \\
H & =m_{R V} \cdot c_{p, C L} \\
\overline{\dot{m}}_{C L} & =k_{C P} \bar{V}_{C P}
\end{aligned}
$$
$[W / K]$ $[W / K]$ $[\mathrm{kg} / \mathrm{s}]$

When the HX is turned off, $A_{C C}$ and $B_{C C}$ become:

$$
\begin{gathered}
A_{C C, \text { of } f}=\left[\begin{array}{cr}
-\frac{F(1-E)+k_{F C}}{G} & \frac{F(1-E)}{G} \\
\frac{F(1-E)}{H} & -\frac{F(1-E)+k_{R V}}{H}
\end{array}\right] \\
B_{C C, \text { off }}=\left[\begin{array}{ccccc}
\frac{1}{G} & 0 & B_{C C, o f f}(1,3) & 0 & \frac{k_{F C}}{G} \\
0 & \frac{1}{H} & B_{C C, o f f}(2,3) & 0 & \frac{k_{R V}}{H}
\end{array}\right]
\end{gathered}
$$

Where $B_{C C, \text { off }}(1,3)$ and $B_{C C, \text { off }}(2,3)$ are given by:

$$
\begin{aligned}
B_{C C, \text { off }}(1,3)= & \frac{c_{p, C L}\left(\bar{T}_{R V}(1-E)-\bar{T}_{F C}(1-E)\right)}{G} \\
& +\frac{\frac{E\left(\bar{T}_{F C}-\bar{T}_{R V}\right) A_{s} \cdot h_{F C}}{\bar{m}_{C L}}}{G}
\end{aligned}
$$




$$
\begin{aligned}
B_{C C, \text { off }}(2,3)= & \frac{c_{p, C L}\left(\bar{T}_{F C}(1-E)-\bar{T}_{R V}(1-E)\right)}{H} \\
& -\frac{\frac{E\left(\bar{T}_{F C}-\bar{T}_{R V}\right) A_{s} \cdot h_{F C}}{\dot{m}_{C L}}}{H}
\end{aligned}
$$

Since $A$ and $B$ are linearised descriptions around an operating point, several $A, B$ matrices have to be calculated depending on the actual operating point. The matrices depend on the following operating parameters:

- $\bar{T}_{C W}$

- $\bar{T}_{R V}$

- $\bar{T}_{F C}$

- $\bar{V}_{C P}$

The cooling system of the FC stack was designed with a temperature difference between stack temperature and cooling liquid inlet temperature of roughly $5^{\circ} \mathrm{C}$. It is therefore assumed that

$$
\bar{T}_{R V}=\bar{T}_{F C}=\bar{T}_{C C}=\frac{1}{2}\left(T_{R V}+T_{F C}\right)
$$

This approach leaves $A_{C C, \text { on }}$ and $A_{C C, \text { off }}$ unchanged and yields for $B_{C C, \text { on }}$ and $B_{C C, \text { off }}$ respectively:

$$
\begin{gathered}
B_{C C, \text { on }}=\left[\begin{array}{cccccc}
\frac{1}{G} & 0 & 0 & 0 & \frac{k_{F C}}{G} \\
0 & \frac{1}{H} & \mathbf{B}_{C C, \text { on }}(2,3) & \frac{F}{H}\left(\epsilon_{0}-k_{x} \overline{\dot{m}}_{C L}\right) & \frac{k_{R V}}{H}
\end{array}\right] \\
B_{C C, \text { off }}=\left[\begin{array}{ccccc}
\frac{1}{G} & 0 & 0 & 0 & \frac{k_{F C}}{G} \\
0 & \frac{1}{H} & 0 & 0 & \frac{k_{R V}}{H}
\end{array}\right]
\end{gathered}
$$

Where $\mathbf{B}_{C C, \text { on }}(2,3)$ is given by:

$$
\mathbf{B}_{C C, \text { on }}(2,3)=\frac{c_{p, C L}\left(\epsilon_{0}-2 k_{x} \overline{\dot{m}}_{C L}\right)\left(\bar{T}_{C W}-\bar{T}_{C C}\right)}{H}
$$

Note that while the heat exchanger is on, a modification of the CL flow rate only affects the reservoir temperature. Through the system's 
cross coupling (i.e. off diagonal terms in (3.44)) this translates into a temperature change of the bipolar plate. Since those off-diagonal terms in $A_{C C, \text { on }}$ also depend on the CL flow rate a change in $\overline{\dot{m}}_{C L}$ will affect all temperatures in the system.

The same is true with the HX turned off where $B_{C C, \text { off }}$ indicates no direct influence of the CL flow rate on the reservoir and fuel cell temperatures. However, the cross coupling inherent to the system as shown in (3.50) depends on the CL flow rate.

\subsubsection{State Space Averaged Overall Cooling Circuit}

The system description outlined in chapter 3.5.7 results in two distinct models depending on whether the chill water valve is open or closed ${ }^{10}$.This section outlines the technique to convert those two distinct state space models into a single model where the user action on the chill water valve appears as a system input.

Because of the large thermal time constants in the cooling system, the chill water valve can be switched at a fixed frequency with the duty cycle adapted to control the system's temperature. In such a configuration, state space averaging theory (see Appendix D) allows an overall system equation to be found. The input vector is extended by the duty cycle and the system matrix is a weighted sum of the system matrices with the HX turned on and off respectively ((3.44) and (3.50)).

$$
\begin{gathered}
\left.\left[\frac{d \tilde{T}_{F C}}{d t}\right] d \frac{d T_{R V}}{d t}\right]=\left[\mathbf{A}_{C C}\right]\left[\begin{array}{c}
\tilde{T}_{F C} \\
\tilde{T}_{R V}
\end{array}\right]+\left[\mathbf{B}_{C C}\right]\left[\begin{array}{c}
\tilde{\dot{Q}}_{\text {loss }} \\
\tilde{\hat{Q}}_{\text {eat }} \\
\tilde{V}_{C P} \\
\tilde{T}_{C W} \\
\tilde{T}_{a m b} \\
\tilde{d}
\end{array}\right] \\
{\left[T_{F C}\right]=\left[\begin{array}{ll}
1 & 0
\end{array}\right]\left[\begin{array}{c}
\bar{T}_{F C}+\tilde{T}_{F C} \\
\tilde{T}_{R V}+\tilde{T}_{R V}
\end{array}\right]}
\end{gathered}
$$

${ }^{10}$ In a configuration where the chill water flow rate can be adjusted continuously through the chill water valve, the system needs to be described by two distinct models because of the discontinuity in heat transfer as the $\mathrm{CW}$ flow rate becomes smaller than the CL flow rate. Two models are also required if a liquid-air type heat exchanger is used in combination with a fan that can only be switched on or off. 
In the case of an air-liquid HX, no state space averaging is required since the fan speed directly acts as a variable control input.

\subsubsection{Summary}

This section has introduced a state-space model to describe the temperature evolution of the fuel cell and the cooling liquid reservoir as a function of ambient conditions and as a function of the user's interaction with the system.

Because the resulting state space equations were non-linear, they have been linearised around a given operating point.

\subsection{Electronic Power Converters}

This section will give a brief overview of the function of electronic power converters. Its scope is not to model such a device but to situate their context. More details on power converters specifically targeted for fuel cell applications can be found in Carlos Rivas' Ph.D. thesis [8].

Fuel cell stacks provide electric power in the form of a certain DC current and voltage. In order for this electrical energy to be useful for driving electric loads (e.g. motors), electric power converters provide a highly dynamic, accurate and flexible way to adapt the voltage, current and frequency levels to the load.

From a controls point of view, electronic power converters act as an input to the fuel cell system by extracting a certain electrical current from the fuel cell. Inherently, electronic power converters are capable of following highly dynamic load conditions. In order not to damage the fuel cell system in the process, feedback from the fuel cell system is required when trying to track a given load profile.

The fuel cell system model elaborated in the context of this thesis provides a means of implementing such a feedback (through model predictive control) without requiring the installation of additional sensors.

Electronic power converters operate by switching at high frequencies (typically from 4-20'000 $\mathrm{kHz}$ ) between different current paths. This allows to modulate the power transfer as desired while generating very few losses. Unfortunately, this produces a high frequency current ripple in the load current withdrawn from the fuel cell. The magnitude of the ripple depends 
on the configuration of the power converter along with the operating conditions.

The description of the high frequency dynamic phenomena that are triggered within the cell by that current ripple is hardly reported in literature and is beyond the scope of this thesis.

A preliminary, model based analysis of the effects of current ripple on the fuel cell operating conditions has been reported by Gemmen et al. [59]. They estimate that to ensure a negligible impact of inverter ripple, the inverter frequencies ${ }^{11}$ need to be above $120 \mathrm{~Hz}$ and have ripple factors of less than $4 \%$.

\subsection{Overall Balance of Plant Model}

The state space models for the different auxiliary systems (i.e (3.8) and (3.10) for the air supply subsystem, (3.14)) and (3.15) for the hydrogen supply subsystem and (3.58) and (3.59) for the thermal management subsystem) can then be easily assembled into a global state space model for the overall balance of plant system.

Adopting the notations outlined in Appendix D, this can be written as:

$$
\begin{aligned}
{\left[\begin{array}{c}
\dot{\mathbf{x}}_{C O} \\
\dot{\mathbf{x}}_{H P} \\
\dot{\mathbf{x}}_{C C}
\end{array}\right]=} & {\left[\begin{array}{ccc}
\mathbf{A}_{C O} & \mathbf{0} & \mathbf{0} \\
\mathbf{0} & \mathbf{A}_{H P} & \mathbf{0} \\
\mathbf{0} & \mathbf{0} & \mathbf{A}_{C C}
\end{array}\right]\left[\begin{array}{l}
\mathbf{x}_{C O} \\
\mathbf{x}_{H P} \\
\mathbf{x}_{C C}
\end{array}\right] } \\
& +\left[\begin{array}{ccc}
\mathbf{B}_{C O} & \mathbf{0} & \mathbf{0} \\
\mathbf{0} & \mathbf{B}_{H P} & \mathbf{0} \\
\mathbf{0} & \mathbf{0} & \mathbf{B}_{C C}
\end{array}\right]\left[\begin{array}{l}
\mathbf{u}_{C O} \\
\mathbf{u}_{H P} \\
\mathbf{u}_{C C}
\end{array}\right] \\
{\left[\begin{array}{l}
\mathbf{y}_{C O} \\
\mathbf{y}_{H P} \\
\mathbf{y}_{C C}
\end{array}\right]=} & {\left[\begin{array}{ccc}
\mathbf{C}_{C O} & \mathbf{0} & \mathbf{0} \\
\mathbf{0} & \mathbf{C}_{H P} & \mathbf{0} \\
\mathbf{0} & \mathbf{0} & \mathbf{C}_{C C}
\end{array}\right]\left[\begin{array}{l}
\mathbf{x}_{C O} \\
\mathbf{x}_{H P} \\
\mathbf{x}_{C C}
\end{array}\right] } \\
& +\left[\begin{array}{ccc}
\mathbf{D}_{C O} & \mathbf{0} & \mathbf{0} \\
\mathbf{0} & \mathbf{D}_{H P} & \mathbf{0} \\
\mathbf{0} & \mathbf{0} & \mathbf{D}_{C C}
\end{array}\right]\left[\begin{array}{l}
\mathbf{u}_{C O} \\
\mathbf{u}_{H P} \\
\mathbf{u}_{C C}
\end{array}\right]
\end{aligned}
$$

\footnotetext{
${ }^{11}$ Typical inverter switching frequencies are several thousand Hertz.
} 
The overall output vector is given by:

$$
\mathbf{y}=\left[\begin{array}{c}
\mathbf{y}_{C O} \\
\mathbf{y}_{H P} \\
\mathbf{y}_{C C}
\end{array}\right]=\left[\begin{array}{c}
\dot{m}_{a i r} \\
p_{a i r} \\
\dot{m}_{H_{2}} \\
T_{F C}
\end{array}\right]
$$

which represent the operating conditions described by the balance of plant model. As mentioned before, the hydrogen pressure is adjusted by the user and then controlled by hydro-mechanical pressure regulator.

The dynamics of the hydrogen pressure, along with the fuel cell inlet humidities in the air and hydrogen supply subsystems have not been modelled in the context of this thesis. From a fuel cell's point of view, they are considered as ambient conditions - along with the ambient pressure - and are accounted for in the fuel cell stack model through direct measurements.

\subsection{Summary}

In the first part of this chapter, a typical PEM fuel cell system with its associated balance of plant systems was shown. Dynamic state-space models were developed for each of the auxiliary systems, i.e. air supply subsystem, hydrogen supply subsystem and thermal management subsystem. Each of those models provides a dynamic description of the effect of a user's interaction with the considered auxiliary component.

Since the auxiliary systems impose the operating conditions for the fuel cell stack, this yields a dynamic description of the FC operating conditions as a function of the user's input.

Experimental results with respect to the models presented in chapters 2 and 3 will be presented in chapter 5 . The test bench used to obtain those experimental results will be described in more detail in chapter 4 . 



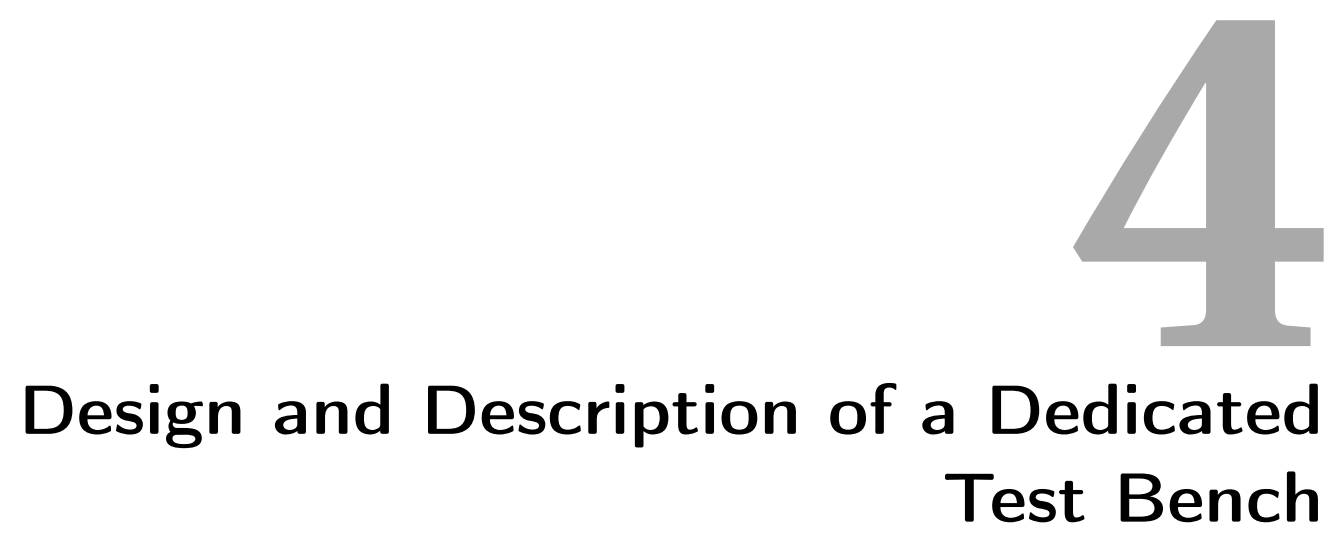




\subsection{Introduction}

In order to be able to experimentally verify the developed models and gain hands-on experience with fuel cell system control, a dedicated FC test bench was developed and built at LEI.

A fuel cell stack jointly developed at the Paul Scherrer Institute (PSI) and the Swiss Federal Institute of Technology in Zürich (ETHZ) was acquired and assembled in-house. Appropriate balance of plant components were selected and installed to provide the FC stack with reactant gases and adjust its operating temperature.

A dedicated hardware and software data acquisition and system control solution was developed and successfully implemented on the test stand.

This chapter aims to give an overview of the experimental setup and provide information about the design choices and implemented solution. Sections $4.2-4.4$ first give a brief overview of the fuel cell stack, the test bench, the data acquisition and control system and then provide more detailed insight into the components' functions and design criteria.

\subsection{Description of the FC stack}

The BRESA FC stack that was acquired from PSI was designed to be used in a fuel cell vehicle $[60,50]$ where oxygen would be provided from ambient air through a compressor and hydrogen would be supplied in compressed gaseous form.

The stack is liquid cooled, provides about $65 \mathrm{~W}$ from each of its $200 \mathrm{~cm}^{2}$ repeat elements. It is designed to operate at $2 \operatorname{bar}(a)$ pressure, a temperature of $70^{\circ} \mathrm{C}$ and air and hydrogen stoichiometry of 2 .

Each carbon bipolar plate is made from two parts that are then glued together. This provides cooling liquid channels within the bipolar plates and assures effective heat removal. The reactant gases enter the stack in a manifold and are then distributed over the cells' surface through meandering gas channels on the anode and cathode side.

E-tek ELAT electrodes and gas diffusion media are used for gas transport to and from the reaction sites and provide a catalyst layer $\left(0.5 \mathrm{mg} \mathrm{Pt} / \mathrm{cm}^{2}\right)$ where the electrochemical reaction takes place. NAFION 112 membranes separate the anode and cathode gas channels and provide a proton conduction medium. More details on the stack and its design can be found in Martin Ruge's Ph.D. thesis [34]. 
To comply with already existing elements of the test bench (particularly the flow meters), 7 repeat elements were acquired from PSI and assembled at LEI to provide a stack delivering about $400 \mathrm{~W}$ under design operating conditions.

The aluminium compression plates as well as the half-bipolar plates at the stack extremities were not available anymore. They were machined according to PSI's drawings at LEI's workshops and Steinemann Carbon $A G$ respectively. The stack was then assembled at $L E I$.

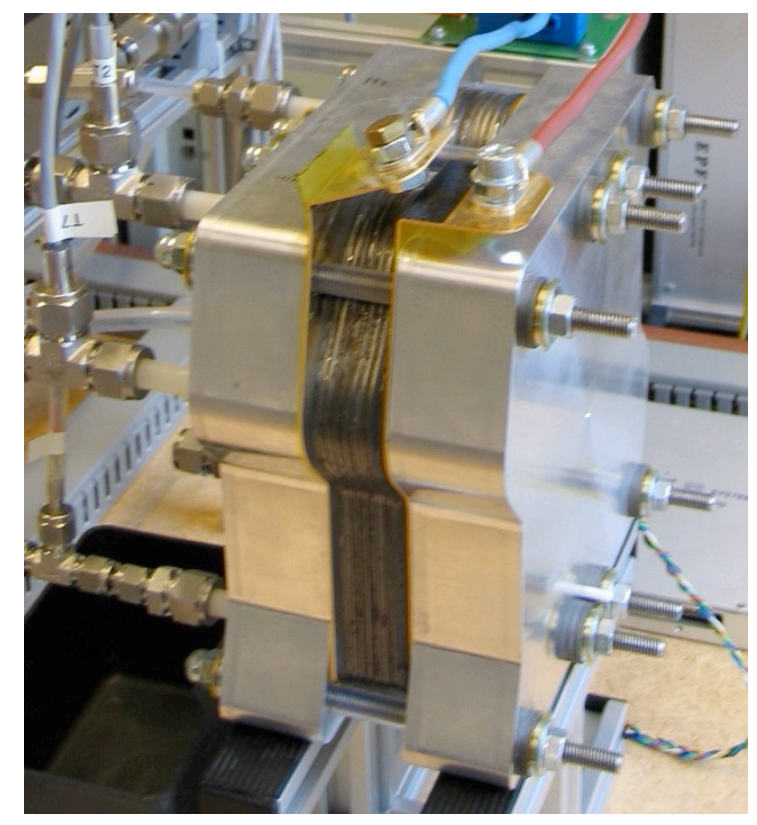

Figure 4.1: Picture of the BRESA FC stack installed at LEI.

The current-voltage characteristics of the stack's first test-run are shown in Figure 4.2. Note that the data was recorded at ambient pressure prior to running the stack in.

\subsection{Description of the Test Bench}

A schematic of the experimental system is shown in Figure 4.3. All sensors are shown by a circle indicating the type of measurement. Inputs that can be controlled by the user are represented by arrows along with a label for the input variable. 


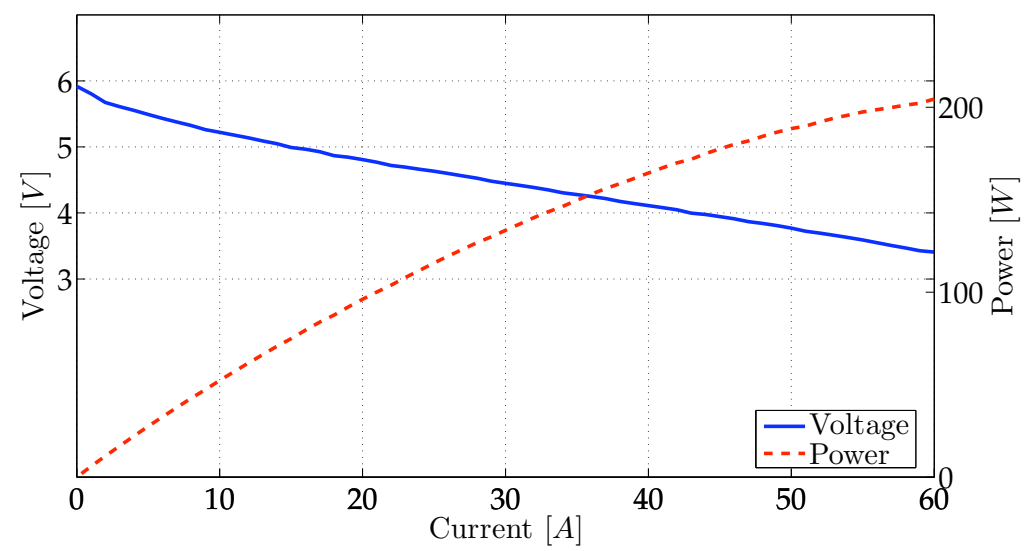

Figure 4.2: IV curve of the stack's first test run with the operating conditions $T_{\text {stack }}=60^{\circ} \mathrm{C}, T_{\text {air }}=70^{\circ} \mathrm{C}$, air dew-point $T_{D P}=60^{\circ} \mathrm{C}$ and non-controlled stoichiometries.

The thermal management subsystem is composed of a cooling liquid recirculation pump, a cooling liquid reservoir with a heating element that allows to either heat the fuel cell to its operating temperature or maintain a cell temperature under low load conditions. A liquid-liquid heat exchanger rejects any waste heat from the cooling liquid circuit to a secondary cooling circuit. The amount of heat rejected through the heat exchanger can be adjusted through the electro-valve $R_{3}$.

The secondary cooling circuit is maintained at around $17^{\circ} \mathrm{C}$ through a liquid-liquid heat exchanger connected to the building's air conditioning circuit. Its cooling liquid is pumped by an independent, mains powered cooling pump and also feeds the water condenser in the hydrogen circuit along with the humidification system.

The air supply subsystem is composed of an air compressor feeding air through a mass flow meter into an air humidification device. Humidity and air temperature can be adjusted independently before the process air enters the fuel cell stack where inlet pressure, temperature and humidity are measured.

The hydrogen supply subsystem is fed with compressed hydrogen between 1 and 2 bar(a) through a manually adjustable pressure reducing valve. Before entering the fuel cell stack the hydrogen's flow rate, pressure, humidity and temperature are measured. At the FC exit, the electro-valve $R_{1}$ either feeds the surplus hydrogen through a condenser or directly to a 


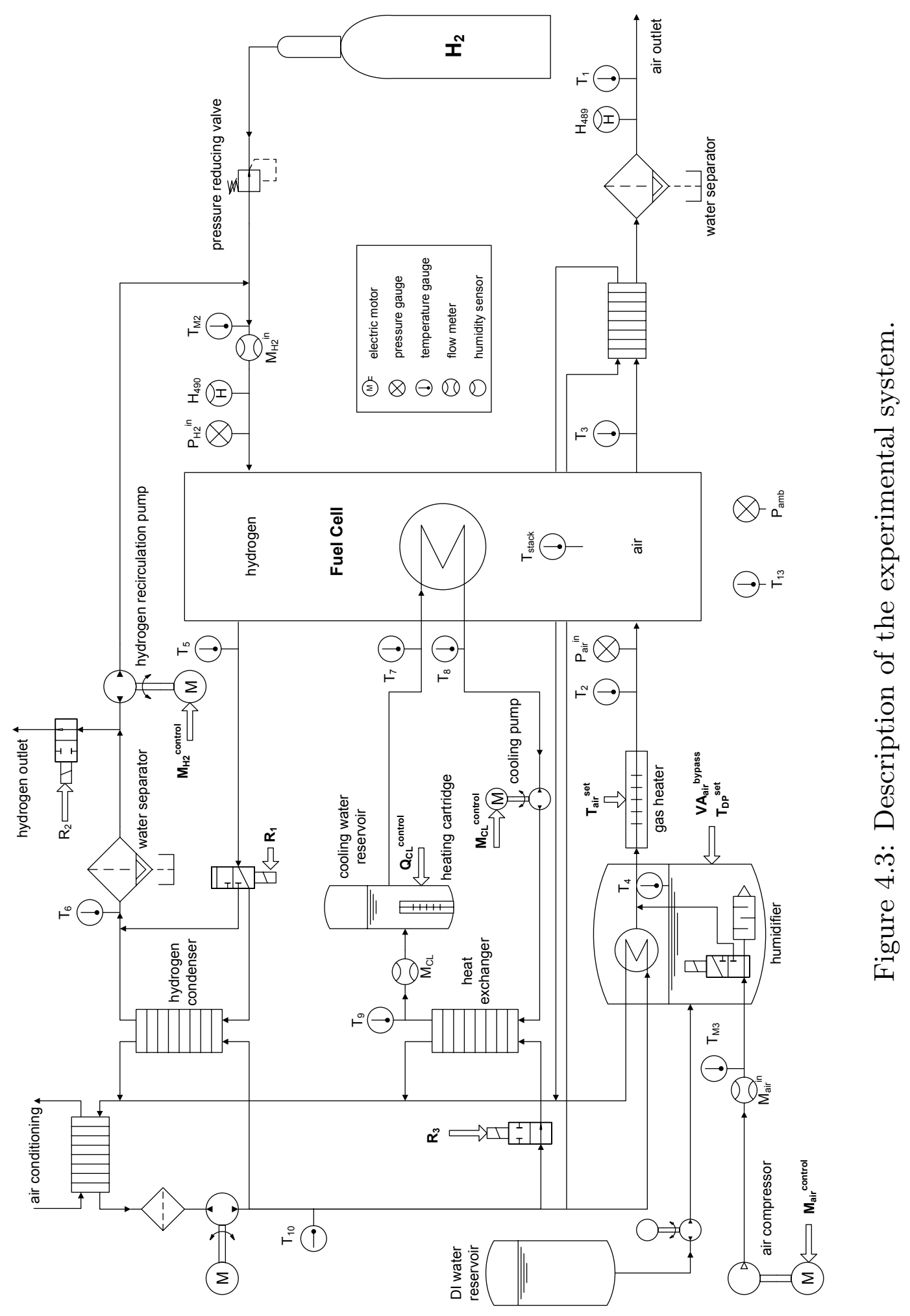


liquid water separator. If passed through the condenser, the hydrogen is cooled to condense water vapour that has accumulated in the gas. From the water separator, the hydrogen is pumped to the fuel cell inlet by a hydrogen recirculation pump. The electro-valve $R_{2}$ allows automated purging of the hydrogen circuit.

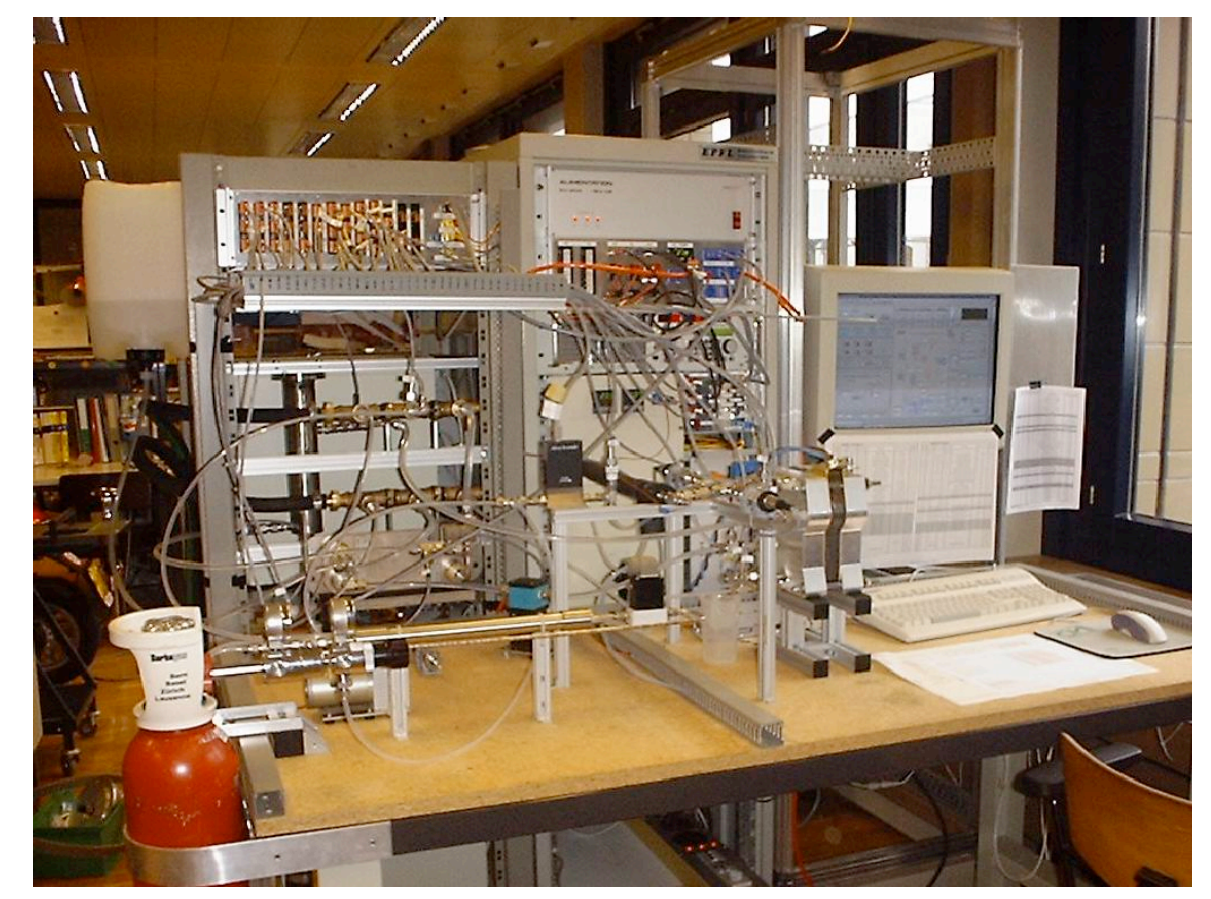

Figure 4.4: Picture of the fuel cell test stand installed at LEI.

The test bench is designed for any water cooled PEM fuel cell stack with an electrical gross power up to $480 W^{1}$ at a average cell voltage of $0.5 \mathrm{~V}$ and stoichiometric flow rates of $\lambda=3$.

\subsubsection{Air Supply Subsystem}

The air supply subsystem is designed to supply the fuel cell stack with humidified air at stoichiometric flow rates of $\lambda_{\text {air }}=3$ at absolute pressures of up to $2 \operatorname{bar}(a)$.

\footnotetext{
${ }^{1}$ The original design goal specified $500 \mathrm{~W}$ of electrical power. This would have required the purchase of new hydrogen and air flow meters. Therefore, the design point of the test bench was derated to $480 \mathrm{~W}$.
} 


\section{Air Compressor}

The design point corresponds to a compression ratio of 2 and a flow rate of 50 slpm (480 W@ $0.5 \mathrm{~V} / \mathrm{cell}$ ). Since PEM fuel cell stacks are very sensitive to impurities contained in the reaction gases, an oil-less compressor with wetted components made from non-corroding materials is required.

An ASF Thomas 2907CDC22 double head membrane compressor was chosen. It is powered by a $12 \mathrm{~V}$ DC motor and supplies a maximum air flow rate of $63 \mathrm{slpm}$ at a relative pressure of $1 \mathrm{bar}(\mathrm{g})$ (see Figure 4.5).

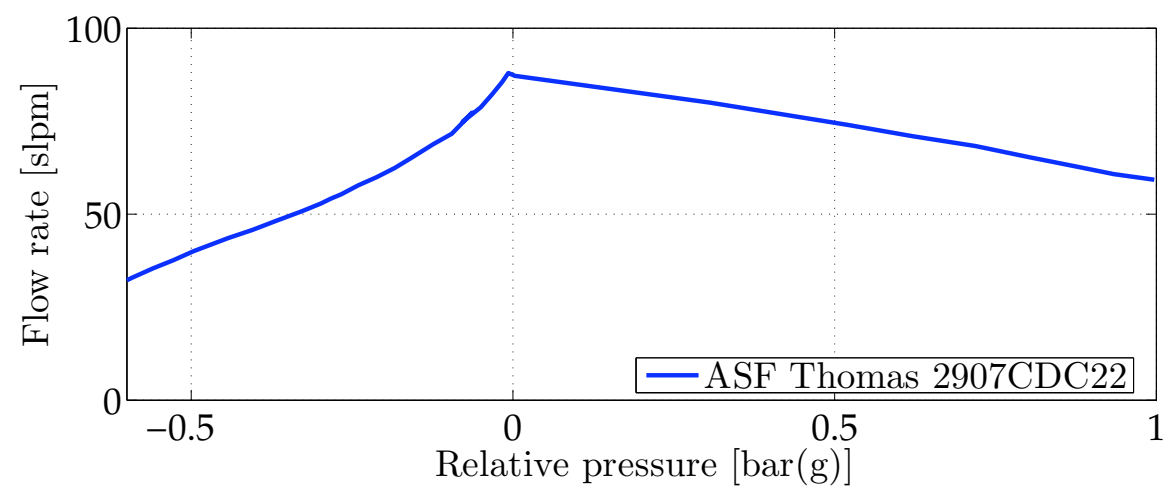

Figure 4.5: Characteristics of the installed ASF Thomas 2907CDC22 double head membrane compressor (manufacturer data).

Because it is an off-the-shelf industrial component, power consumption is rather high. The manufacturer specifies an electrical power consumption of $240 \mathrm{~W}$ at a compression ratio of 2 and a flow rate of $63 \mathrm{slpm}$ corresponding to an overall isentropic efficiency of $\eta_{K s} \approx 1 \%$. It is thus unsuitable for stand-alone fuel cell systems where the auxiliaries are powered by the fuel cell but proved adequate for a test bench environment.

\section{Air Humidification System}

In order to run the fuel cell system at any desired humidity, an Arbin DPH humidifier is installed downstream from the air compressor.

The schematic of the humidifier system is shown in Figure 4.6. A humidifier core $(D P H)$ is filled with deionised water from a reservoir and electrically heated to the desired dew point temperature. The process air is fed through the humidifier core where it takes up water. If this is not 
desired, a bypass valve allows air to be directly fed to the exit heater that adjusts the air temperature to the chosen exit temperature.

Chill water is required for the core to operate correctly. Additionally, it serves as a heat transfer medium in the exit heater. Its flow can be adjusted through a manual flow regulator (denoted as "Black Flow Regulator") large flow rates allow fast reductions of the humidifier core and exit heater but decrease the speed with temperature can be increased. This is shown in Figure 4.7 where the chill water flow rate is increased to $50 \mathrm{cl} / \mathrm{min}$ for negative changes in set-point temperature.

A second manual flow regulator (denoted "Transparent Flow Regulator") feeds chill water through a concentric tube heat exchanger that cools the chill water from the exit heater outlet.

Both electrical heating elements are powered by electronic temperature controllers whose set-points are manually adjusted by the user. An additional temperature sensor has been integrated into the system to monitor the dew point temperature $T_{D P}$ from the data acquisition system.

Figure 4.7 shows the dynamic response of the humidifier system to a change in set-point temperature. The time constants for increasing temperatures are given by available heating power compared to the thermal inertia. For the $D P H$ core, time constants of $\tau_{D P H, u p} \approx 12 \mathrm{~min}$ are observed. For the exit heater, they are around $\tau_{E H, u p} \approx 4 \mathrm{~min}$. When reducing the desired temperature, the chill water flow determines the resulting temperature. On the measurements in Figure 4.7, the flow rate was increased to $50 \mathrm{cl} / \mathrm{min}$ resulting in time constants around $\tau_{\text {decrease }} \approx 2 \mathrm{~min}$.

\subsubsection{Hydrogen Supply Subsystem}

The hydrogen supply subsystem is designed to supply the fuel cell stack with hydrogen at stoichiometric flow rates of $\lambda_{H_{2}}=3$ at absolute pressures up to $2 \operatorname{bar}(a)$. No external hydrogen humidification is required - the hydrogen gets humidified in the fuel cell. Since the hydrogen supply circuit is closed, the acquired humidity accumulates within the system. In order to regulate the hydrogen humidity, a switchable water condenser is installed in the system.

\section{Pressure Regulator}

The pressure regulator supplies hydrogen at the desired pressure to the hydrogen manifold. It interfaces a high pressure $(20-250 \operatorname{bar}(g))$ hydrogen 


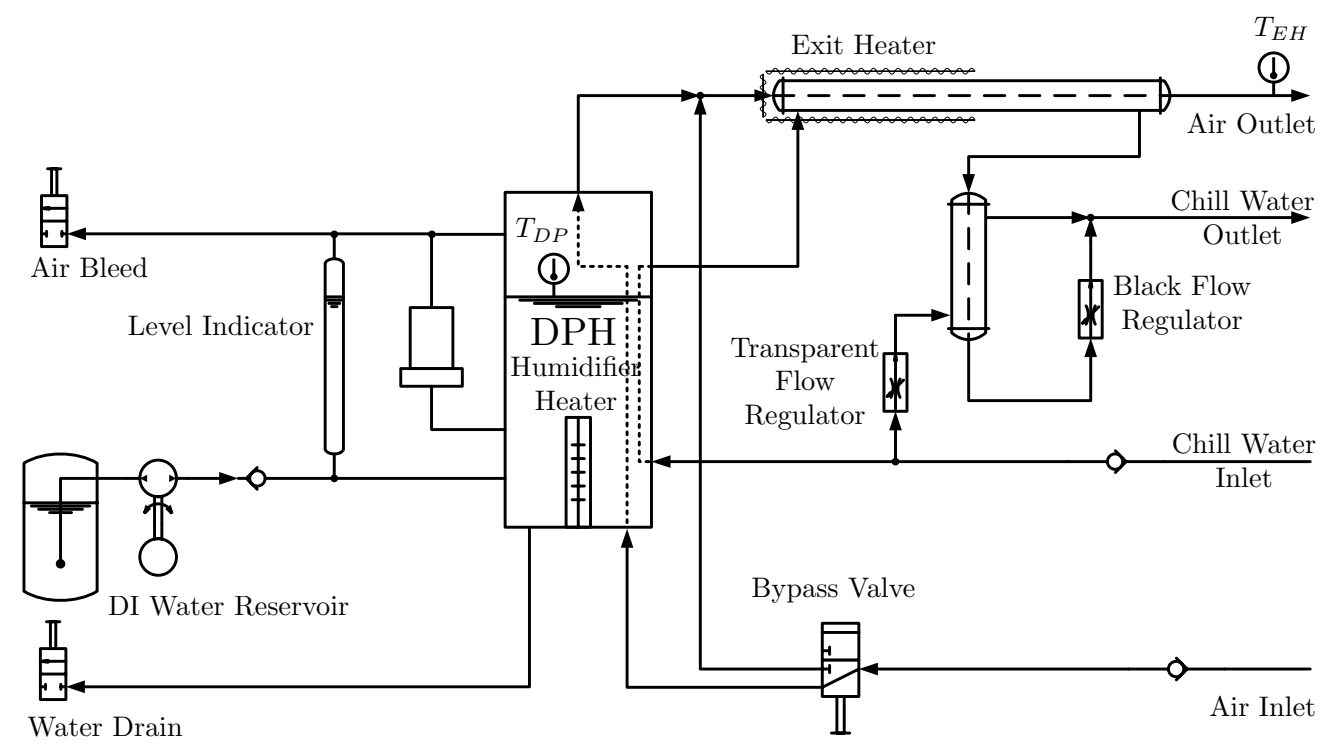

Figure 4.6: Schematic representation of the Arbin DHP humidification system.

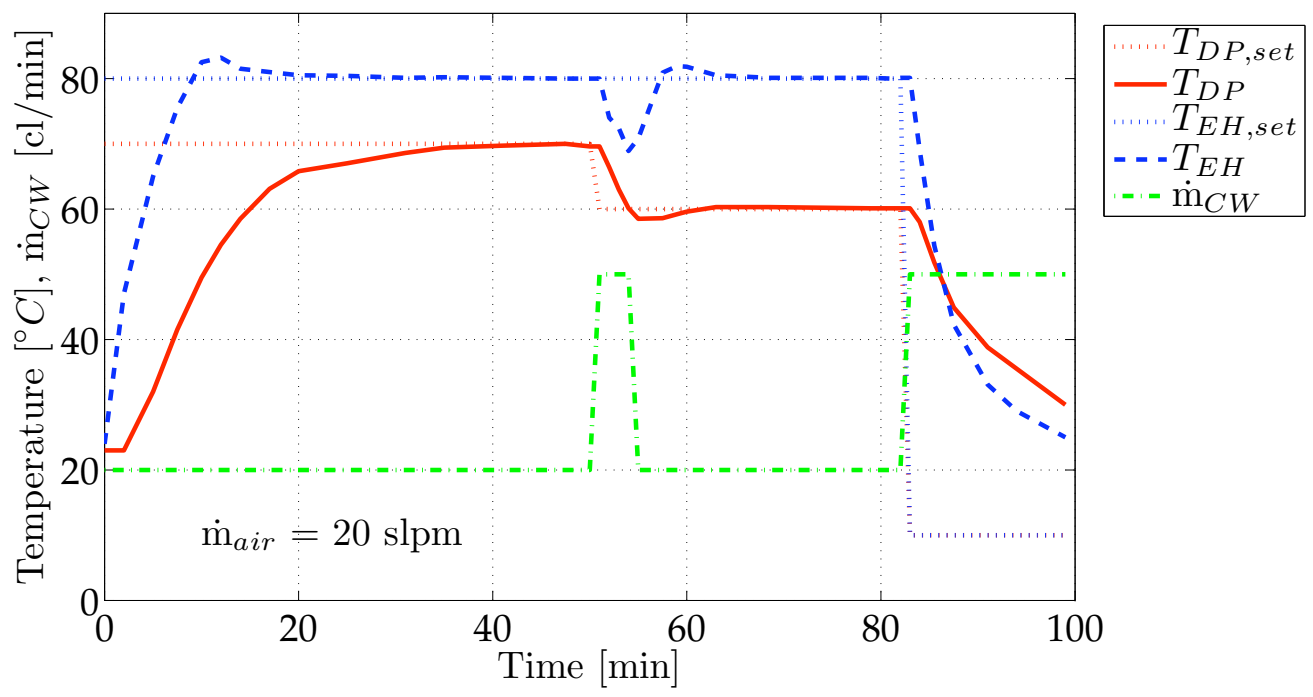

Figure 4.7: Experimental temperature evolution of the dew point $\left(T_{D P}\right)$ and exit heater $\left(T_{E H}\right)$ temperatures. 
container to the fuel cell and automatically adjusts the hydrogen flow rate so as to keep the desired pressure within the hydrogen manifold.

An Air Liquide HBS 315/1 pressure regulator is installed on the test bench. It is capable of maintaining a user adjusted pressure level between 0 and $1 \operatorname{bar}(\mathrm{g})$ for flow rates below $20 \mathrm{slpm}$. If the flow rates increase any further, the pressure level cannot be maintained any more and drops (see Figure 4.8). Because the pressure regulator only needs to supply the effectively consumed hydrogen (i.e. $\lambda_{H_{2}}=1$ ) - corresponding to $6 \mathrm{slpm}$ of hydrogen at the test bench design point of $480 \mathrm{~W} @ 0.5 \mathrm{~V}$ - the desired operating pressure can be effectively maintained.

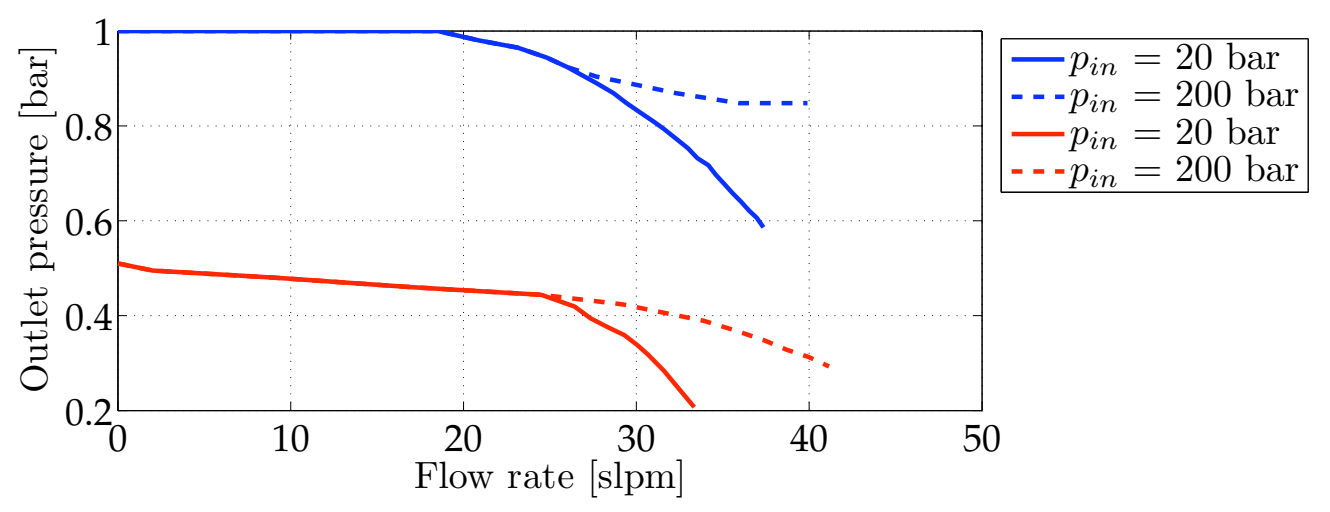

Figure 4.8: Characteristics of the Air Liquide HBS 315/1 spring loaded pressure regulator (manufacturer supplied data).

The dynamics of the pressure regulator are difficult to model. They have been outlined in chapter 3.4.3 where experimental data shows the dynamic response of the pressure regulator to a sudden change in pressure in Figure 3.8 on page 76.

\section{Water Condenser and Separator}

Water can migrate through the fuel cell from the cathode (where it is produced by the electrochemical reaction) to the anode compartment and back. In order to regulate the hydrogen humidity in the hydrogen supply circuit, a water condenser and separator system has been designed.

The water condenser is a concentric tube heat exchanger where the outside compartment is fed with chill water maintained around $17^{\circ} \mathrm{C}$. It is designed to cool $100 \%$ humid hydrogen at $60{ }^{\circ} \mathrm{C}$ to $30^{\circ} \mathrm{C}$ while transferring 
enough heat to condense the water vapour to an exit dew point of $30^{\circ} \mathrm{C}$. It is manufactured from stainless steel to avoid any corrosion in the system.

A custom made water separator eliminates any liquid water from the fuel stream before the hydrogen is re-circulated by the hydrogen re-circulation pump. A transparent water level indicator allows to easily determine the amount of condensed water in the system.

\section{Condenser Bypass Valve}

The hydrogen humidity can be adjusted by either feeding the surplus hydrogen from the fuel cell outlet through the water condenser or bypassing it. The Bürkert Type 124 condenser bypass $2 / 3$ way valve is made from poly-propylene with a minimum orifice opening diameter of $4 \mathrm{~mm}$. The solenoid actuator is powered by $220 \mathrm{~V}$ AC and can be automatically switched from the data acquisition and control system. Under no power conditions, hydrogen is directed through the water condenser.

\section{Hydrogen Recirculation Pump}

The hydrogen recirculation pump needs to establish a hydrogen flow rate of $20 \mathrm{slpm}$ at the design point. Since the hydrogen supply circuit is pressurised by the pressure regulator, the pump only needs to overcome the pressure drop from the fuel cell, the water condenser and the condenser bypass valve. The overall pressure drop has been evaluated at $\delta p_{H_{2}} \leq 0.3 \mathrm{bar}$.

As with the air compressor, purity considerations require an oil-less pump with wetted components made from non-corroding materials.

A $K N F N 828 K N D C$ single head membrane pump is installed on the test bench. It is powered by a $24 \mathrm{~V}$ DC motor and supplies a maximum hydrogen flow rate of $5 \mathrm{slpm}$ against a relative pressure difference of $1 \operatorname{bar}(g)$. At the design point of $0.3 \operatorname{bar}(g)$, the flow rate is approximately 20 slpm (see Figure 4.9).

The hydrogen pump has been conceived as a low-cost, laboratory pump. Therefore, its power consumption is high ( $40 \mathrm{~W}$ at maximum pressure and flow rate) and makes the pump unsuitable for stand-alone fuel cell systems where the auxiliaries are powered by the fuel cell but proves adequate for a test bench environment. 


\subsection{DESCRIPTION OF THE TEST BENCH}

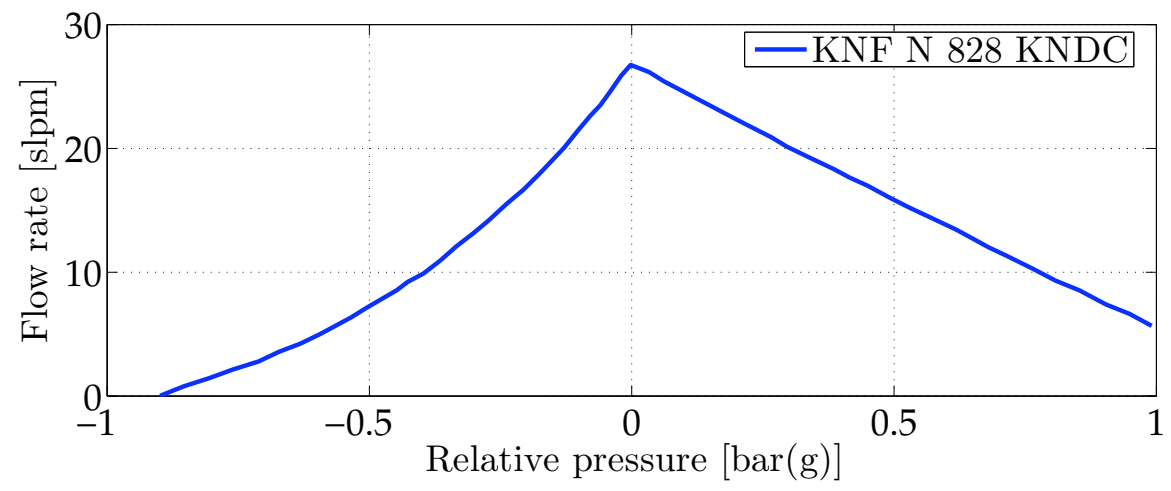

Figure 4.9: Characteristics of the installed KNF $N 828$ KNDC single head membrane pump (manufacturer data).

\section{Hydrogen Purge Valve}

In order to easily purge the hydrogen supply circuit, a dedicated purge valve is installed on the test bench. It is a Bürkert Type 6011 2/2 solenoid valve made from stainless steel, capable of withstanding a pressure difference up to $6 \operatorname{bar}(g)$. It is powered by $220 \mathrm{~V}$ AC and can be automatically switched from the data acquisition and control system. Under no power conditions, the valve is closed by a pre-tensioned spring.

\subsubsection{Thermal Management Subsystem}

The thermal management is designed to maintain the fuel cell at any temperature between 45 and $80^{\circ} \mathrm{C}$ regardless of the fuel cell's operating point. This means that the system must be able to maintain the $\mathrm{FC}$ at $80^{\circ} \mathrm{C}$ under no load conditions and be capable of rejecting its waste heat of $700 W^{2}$ at $45^{\circ} \mathrm{C}$.

Because the cooling liquid is in direct contact with all cells of the stack, it cannot be electrically conductive. Therefore, deionised water is chosen as a cooling liquid and all elements within the cooling circuit must be made of non-corroding materials to maintain the water in its deionised state.

\footnotetext{
${ }^{2}$ This corresponds to the waste heat produced at the design point of $480 \mathrm{~W}$ at $0.5 \mathrm{~V}$.
} 


\section{Cooling Liquid Reservoir and Heating Element}

A cooling liquid reservoir was designed to provide a cooling liquid reserve to compensate for any evaporation, to function as an air purging device and to operate as a cooling liquid heater. It is made from stainless steel, provides an optical fill level indicator and contains about $0.6 l$ of deionised cooling liquid.

An integrated Watlow $660 \mathrm{~W}$ electrical heating cartridge is powered by $220 \mathrm{~V}$ AC through a dedicated temperature controller. The temperature controller's set-point temperature is adjusted manually. The heating cartridge can also be directly connected to one of the data acquisition and control system's $220 \mathrm{~V}$ solid state relays. The cooling liquid temperature can then be computer controlled from the test stand's control interface.

\section{Cooling Liquid Recirculation Pump}

The cooling liquid recirculation pump needs to establish a cooling liquid flow through the fuel cell and heat exchanger so that the operating temperature can be maintained. The fuel cell's design [34] requires a minimum flow rate of $3.2 \mathrm{~g} / \mathrm{s}$ per cooling channel - for the 7-cell stack (8 cooling channels) installed at LEI, this translates into a minimum overall cooling liquid flow rate of $1.5 \mathrm{lpm}$. This flow has also been adapted as the design point for the heat exchanger.

The maximum pressure drop in the cooling circuit is estimated at around 80 mbar for the cooling channels in the fuel cell and around 8 mbar in the heat exchanger.

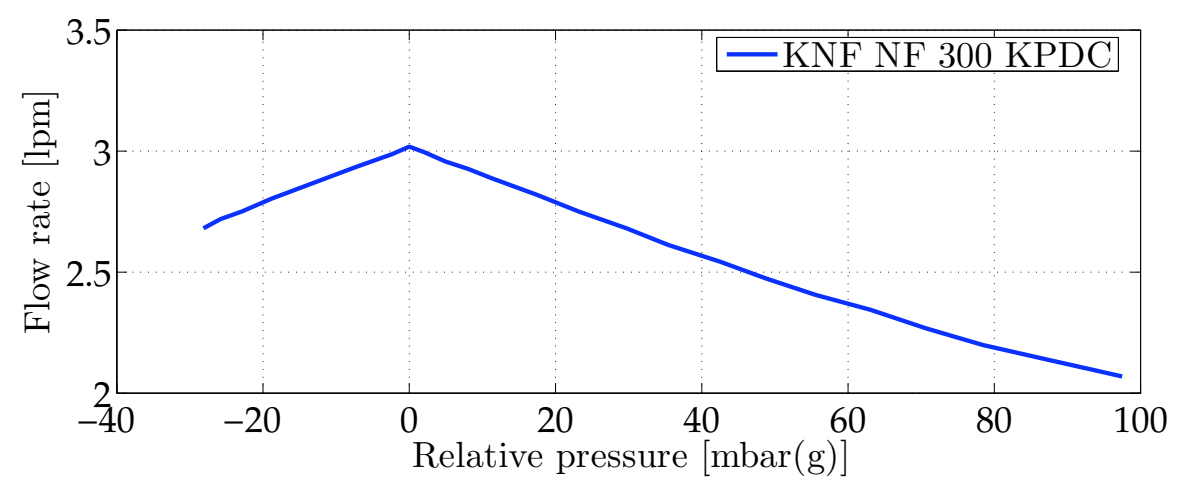

Figure 4.10: Characteristics of the installed KNF $N 300$ KPDC single head membrane pump (manufacturer data). 
A $K N F N 300 K P D C$ single head membrane pump is installed on the test bench. It is powered by a $24 \mathrm{~V}$ DC motor and supplies a maximum cooling liquid flow rate of $2.2 \mathrm{lpm}$ against a pressure drop of $80 \mathrm{mbar}$ (see Figure 4.10). Power consumption at the design point is around $35 \mathrm{~W}$.

\section{Primary Circuit Heat Exchanger}

The primary circuit heat exchanger has been dimensioned to reject the maximum waste heat with a cooling liquid inlet temperature of $45^{\circ} \mathrm{C}$ and a chill water inlet temperature of $17^{\circ} \mathrm{C}$. A Funke TPL 00-K-4-22-1.1 plate heat exchanger made from stainless steel is installed on the test bench. It is capable of rejecting $750 \mathrm{~W}$ of heat at the design point. As the fuel cell temperature increases, the heat transfer capacity increases linearly to a maximum of $1730 \mathrm{~W}$ at $T_{F C}=80^{\circ} \mathrm{C}$.

\section{Secondary Circuit Chill Water Pump}

The secondary circuit chill water pump needs to supply chill water to the heat exchanger, to the water condenser in the hydrogen supply subsystem and to the air humidifier. All circuits are connected in parallel to a chill water distribution manifold - the flow in each circuit is determined by its flow resistance.

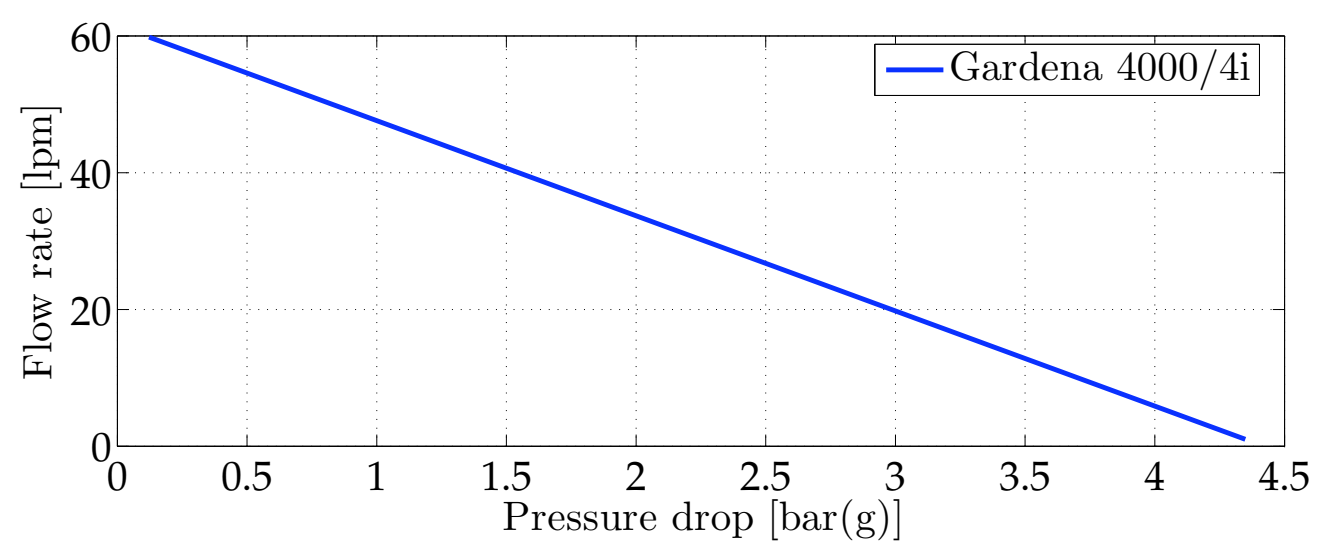

Figure 4.11: Characteristics of the Gardena $4000 / 4 i$ water pump (manufacturer data).

A Gardena 4000/4i water pump has been installed on the test bench. It is capable of supplying chill water up to $60 \mathrm{lpm}$ under no pressure drop 
conditions. For the equipment installed on the LEI test bench, the overall flow rate is around $20 \mathrm{lpm}$. The pump is powered by $220 \mathrm{~V}$ AC and can be automatically switched on and off from the data acquisition and control system.

\section{Chill Water Valve}

To control the chill water flow through the primary heat exchanger, a solenoid shut-off valve has been installed upstream from the primary circuit heat exchanger. The valve is a Bürkert Type 0211B $2 / 2$ solenoid valve made from brass. It provides little flow resistance through its opening diameter of $3 \mathrm{~mm}$ while withstanding a pressure difference up to $6 \operatorname{bar}(g)$. It is powered by $220 \mathrm{~V}$ AC and can be automatically switched from the data acquisition and control system. Under no power conditions, the valve is opened by a pre-tensioned spring to make sure the system can still be cooled in case of a power or solenoid failure.

\section{Secondary Circuit Heat Exchanger}

A secondary circuit heat exchanger is connected to EPFL's air conditioning system to reject all waste heat from the FC test bench. Chill water flow is provided by the chill water pump at flow rates around $20 \mathrm{lpm}$. The Isolux WP3-50 stainless steel plate heat exchanger has a rated power of $10 \mathrm{~kW}$ allowing isothermal chill water conditions around $17^{\circ} \mathrm{C}$ regardless of the system's operating conditions.

\subsection{Description of the Data Acquisition and Control System}

To control and supervise the test bench, a data acquisition and control system has been designed. Particular emphasis was placed on being able to record system transients. It is centred around a PC equipped with two data acquisition cards that are controlled by a specially designed LabView data acquisition and control program.

All the sensors are connected to and powered by a data acquisition interface rack where the signals are adapted to the DAQ cards' requirements. The sensor data is acquired in real time and streamed to text files to be used for model identification and verification. Dedicated digital to analog 
conversion channels along with digital signal outputs are used to control the test bench and fuel cell system.

This chapter will focus on the sensors and actuator design and selection in a first part before describing the data acquisition and control software in more detail.

\subsubsection{Data Acquisition and Control Hardware}

\section{Data Acquisition Boards}

A National Instruments NI 6014 and NI PCI 16 MIO DAQ card is installed in the test bench PC. They acquire sensor data through 16 16-bit and 12-bit AD channels respectively and output set-point signals through a total of four DA channels.

8 digital I/O lines are available per device. They are used to control the solid state relays for switching $220 \mathrm{~V}$ powered equipment and for powering three additional low resolution $\mathrm{D} / \mathrm{A}$ outputs through a set of multiplexers located on the interface cards.

Each card is equipped with 724 -bit and 3 16-bit counters that are used for frequency measurements of pulse signals and allow synchronisation of several DAQ cards.

The NI PCI 16 MIO card is capable of sampling each of the 16 input channels at $16 \mathrm{kS} / \mathrm{s}$ while the NI 6014 provides a sampling rate of $12 \mathrm{kS} / \mathrm{s}$. Both cards are piloted through a custom designed LabView interface.

Additionally, a National Instruments AT-GPIB/TNT GPIB interface card allows the data acquisition and control software to communicate with devices on the GPIB bus. Currently, this is the case for the electronic load.

A Smart GmbH PCC-PCI interface card provides data exchange between the LabView data acquisition and control program and external devices on the $C A N$ bus (i.e. the cell voltage monitoring system).

\section{Temperature Measurements}

Temperature measurements are acquired through PT 100 resistance temperature detectors (RTD). To guarantee fast response times, the sensing elements have been custom fit to Swagelok fittings. Smaller, and therefore faster sensors were chosen for gas temperature measurements while larger, more robust elements have been implemented for liquid temperature measurements. 
Typical response time constants is around $\tau_{P T 100, \text { gas }}=120 \mathrm{~ms}$ for the gas temperature sensors and around $\tau_{P T 100, \text { liquid }}=400 \mathrm{~ms}$ for the liquid temperature sensors. The sensors are connected to interface cards where their signal is transformed into a standard 4-20 $\mathrm{mA}$ output signal through a Burr Brown XTR 105 integrated circuit.

\section{Pressure Measurements}

Three Keller PR-33 piezoresistive pressure sensors have been installed on the test bench. They monitor the air and hydrogen inlet pressure along with the ambient pressure. The air and hydrogen sensors are calibrated for relative pressures from $0-1 \operatorname{bar}(g)$ while the ambient pressure sensor monitors absolute pressures from 0.8-1.2 $\operatorname{bar}(a)$ - both with accuracies of $\pm 0.5 \%$ of full scale.

The sensors operate in a temperature range between $-10-80^{\circ} \mathrm{C}$. The $0-$ $10 \mathrm{~V}$ output signal is generated by an internal DA converter at a frequency of $100 \mathrm{~Hz}$. A manufacturer-supplied software allows to monitor and reprogram the sensors through a $R S 485$ connection.

The sensors are connected to interface cards providing power and directing their signals to the data acquisition cards.

\section{Flow Measurements}

The test stand comprises three flow metering devices, monitoring the air and hydrogen flow rates along with the cooling liquid flow. The air and hydrogen flow meters allow to get data fast enough to resolve transient operating conditions. The cooling liquid flow meter is only suitable for steady state measurements.

Two Alicat Scientific $M$ series mass flow meters monitor the air and hydrogen flow rates respectively. The mass flow rate is calculated from pressure drop readings across a laminar flow element within the flow meter along with gas temperature measurements. Because this measurement technique does not involve the thermal inertia inherent to thermal mass flow meters, response time is very fast at around $10 \mathrm{~ms}$.

The mass flow rates and temperatures are output through $0-10 \mathrm{~V}$ analog connectors with an accuracy of $\pm 1 \%$ of full scale. The air flow meter is calibrated for flow rates from 0-50 slpm while the hydrogen flow meter's range is from $0-20 \mathrm{slpm}$ - temperature sensor range is from $0-80^{\circ} \mathrm{C}$. The 
flow meters feature an $R S$ 232 connector allowing data collection by any PC.

A Gems Sensors FT-110 Series turbine flow rate sensor provides a cooling liquid flow rate measurement. The sensor has an operating range from $0.5-5 \mathrm{lpm}$ with an accuracy of $\pm 3 \%$ of full scale. The measurement is output as frequency modulated pulse signal that is connected to the DAQ cards through the interface rack.

\section{Humidity Measurements}

Honeywell HIH-3602-C and HIH-3610-002 humidity sensors are used to monitor hydrogen and air humidities. They provide an analog output voltage proportional to the relative humidity with an accuracy of $\pm 2 \% R H$. Additionally (for the $H I H-3602-C$ type), an integrated PT 1000 RTD provides the local temperature.

\section{Voltage Measurements}

Compressor, pump and fuel cell voltages are acquired through a custom made interface card featuring a differential instrumentation operational amplifier (Burr Brown INA 117) per channel. The amplifiers' output signals are fed to the DAQ cards.

\section{Single Cell Voltage Measurements}

Individual FC cell voltages are acquired through a dedicated Smart GmbH $C V M$ system. Up to 80 cells with individual voltages up to $\pm 3 V$ can be monitored simultaneously. The cell voltages are transmitted through a $C A N$ bus that is connected to the host computer through a PC-CAN interface card.

Since signal transmission and voltage measurements cannot be synchronised with the real time data acquisition system, a fuel cell current mirror signal is read simultaneously with the cell voltages. This allows the CVM data to be time correlated with the other measurements from the test bench. 


\section{Current Measurements}

Compressor, pump and fuel cell voltages are acquired through a set of galvanically isolated $L E M$ current transducers. Their measurement circuit load resistance is integrated into the respective interface card from where the output signal is connected to the DAQ cards.

\section{V AC Switching Interface}

A set of six Cosmo KSD203AC2 solid state relays can be switched through the digital I/O channels provided by the DAQ cards. They switch alternative current, single phase loads up to $750 \mathrm{~W}$. Turn-on and off times are typically around $8 \mathrm{~ms}$.

\section{Pump Motor Controllers}

The cooling liquid and hydrogen pumps are each powered by a Portescap ELD-3503-V4 motor controller capable of supplying up to $2.5 \mathrm{~A}$ continuous current. They are used in transconductance mode where a motor current is generated based on the set-point input signal from the DAQ cards. Power supply to the motor controllers is through a $24 \mathrm{~V}$ DC laboratory power supply.

In order to handle the current levels of the air compressor motor, a custom designed H-bridge motor controller has been developed at LEI. It is powered from a $12 \mathrm{~V}$ laboratory power supply and adjusts the motor voltage to the level specified by a set-point input signal.

\section{Electronic Load}

The fuel cell load is simulated by an TDI Dynaload $R B L-488$ electronic load. It is controlled from the LabView interface through a GPIB bus. The load can be operated in constant current, constant resistance, constant voltage or constant power mode.

For each load mode, the set-point value is transmitted through the $I E E E-488$ bus. If a particular load profile is desired, an analog, external reference input can be provided or - for pulse loading - an integrated pulse load mode can be activated.

The load is capable of sinking a maximum electrical power of $2000 \mathrm{~W}$ with currents up to $300 \mathrm{~A}$ and voltages up to $100 \mathrm{~V}$. 


\subsection{DATA ACQUISITION AND CONTROL}

\section{Temperature Controllers}

A Watlow Series 935A temperature controller is used to set and regulate the cooling liquid temperature through the heating cartridge in the cooling liquid reservoir. The controller features an external set-point input so that it can be connected to the data acquisition and control system. This allows automatic adjustment of the set-point temperature depending on the operating conditions.

The Arbin air humidification system features two RKC CB 100 temperature controllers to set and control the air dew point and exit temperatures. The controllers do not provide a set-point input channel - the desired dew point and exit temperatures therefore have to be adjusted manually.

\section{Manual Control Interface}

For manual control of the fuel cell or to carry out emergency shut down procedures, a manual control interface has been designed.

A master switch changes from manual to automatic system control. In manual mode, the set-point and control signals are manually adjusted on the front panel, in automatic mode, the set-point and control signals generated by the LabView data acquisition and control software are transmitted to the test bench.

In manual mode, the air compressor, cooling liquid and hydrogen pump speeds are manually controlled through potentiometers. Individual toggle switches directly change the state of the 6 solid state relays. Additional switches turn the electronic load on or off and tare the mass flow meters.

\subsubsection{Data Acquisition and Control Software}

A dedicated data acquisition and control software was developed in LabView. It allows automatic control of the test bench and fuel cell while streaming all sensor data to disk for later analysis.

The system can be run in two different modes depending on what type of phenomena needs to be observed:

Closed loop mode provides data acquisition and system control at the same time. The available system bandwidth limits the sampling frequency to about $100 \mathrm{~Hz}$. 
The air flow rate, the cooling liquid and hydrogen flow rates are adjusted through software implemented, closed loop, PID controllers.

The test bench sensor data is read individually at each sampling period. This permits synchronisation between data acquired by the sensors and data acquired through other means (i.e. CAN bus, counters, etc.). Therefore, all the data acquired (see chapter Appendix E.2) is recorded to disk.

Open loop mode provides data acquisition without system control (i.e. open loop control of the auxiliary systems). In this mode, faster phenomena (up to $1 \mathrm{kHz}$ ) can be captured.

The variables indicated as user controllable in Figure 4.3 are adjusted by the user (either through manual controls or by fixing their values on the LabView interface) but cannot be controlled in a closed loop because of limited system resources.

The test bench sensor data is buffered in the DAQ cards and uploaded to the software in batches. As a result, no synchronisation between data acquired by the sensors and data acquired through other means is possible. Therefore, the state of the digital I/O channels, the state of the DA output channels, the cell voltages and the cooling liquid flow rate are not recorded to disk. They are, however, still displayed on the user interface.

Open loop mode is only to be used for short periods of time to monitor specific fast transient phenomena.

After start-up, the system runs through an initialisation routine during which, feedback is provided to the user as to which steps have been successfully completed. First the PC- $C A N$ interface driver is loaded. Upon completion, communication with external instruments is established on the $C A N$ and GPIB buses. Additionally, the "blackbox" file to which sensor data is written periodically is loaded into memory and a new header row with the current data and time is written to the file.

Once the start-up procedure is completed, the system is in run mode where the test bench is run and monitored by the system. The functionality that is available to the user in run mode is described more in detail in the section entitled "Functionality" on page 116.

Upon a shut-down command issued by the user, the system closes the "blackbox" file and saves it back to disk and terminates communication 
on the $C A N$ and GPIB buses. During the whole procedure, the user is informed about the state of the shut-down routine.

\section{Functionality}

This section explains more about the functionality provided by the data acquisition and control software. It focuses on what is available in closed loop mode since that is the intended use of the test bench.

Data acquisition Appendix E.2 lists the variables measured by the data acquisition and control system. They are displayed on screen and can be streamed to disk for later usage. The first 32 measurements are acquired through the $\mathrm{AD}$ converters of the $\mathrm{DAQ}$ cards. The cooling liquid flow rate $m_{C L}$ is determined through an DAQ card internal counter - its value is only updated at a rate of $2 \mathrm{~Hz}$. The individual cell voltages are acquired through the $C A N$ bus connected to the host PC.

Normally, the variables are not directly written to disk (apart from the black box mode mentioned on page 117). However, the user can initiate a data acquisition mode. While in this mode, all data gets streamed to disk at the user specified data acquisition frequency. For each time data acquisition mode is started, a new measurement file is created.

Data display Under normal test bench operation, the variables are filtered with a user adjustable time constant (typically set to $\tau_{\text {display }}=0.5 \mathrm{~s}$ and then displayed as numerical values in the user interface on screen. Additionally, characteristic operating parameters (i.e. power, stoichiometric flow rates) are derived from the measurements and displayed on screen.

The data is displayed on a schematic representation of the fuel cell system in order to facilitate data recognition. An additional bar graph diagram provides real-time feedback of all cell voltages in the system.

IV curve creation An IV curve creation mode automatically runs the fuel cell from no load conditions to a predefined maximum current and back. The current steps, maximum current and time step between each measurement point is user adjustable. 
The operating conditions (i.e. stoichiometric flow rates, temperature, etc) are maintained at the values set before starting the IV curve.

Black box functionality While the test bench data acquisition and control software is running, all variables are written to a dedicated black box file every 10 seconds. This provides automated long term monitoring of the test bench and allows identification of abnormalities during operation.

Load programming The electronic load (see section 4.4.1) is programmed and supervised through a GPIB bus. The user can adjust the load mode (i.e constant current, voltage, power or resistance), the duty cycle load parameters along with the desired load. Additional interface elements connect and disconnect the electronic load from the fuel cell and initiate pulse mode.

System Control Through the interface, the user can directly switch the solenoid valves installed on the test bench. The chill water valve is switched automatically by a temperature control loop that monitors the cooling liquid temperature at the fuel cell outlet. If the temperature exceeds the maximum value specified by the user, the chill water valve is opened to cool the fuel cell.

The air and hydrogen flow rates are specified by the user as a certain minimum flow rate to be maintained along with a desired stoichiometric flow rate. The cooling liquid flow rate is directly specified by the user. Independent, software based PID controllers then maintain the flow rates at the specified values.

The following variables are controllable manually (i.e. not through the LabView interface), but their states are measured and recorded.

1. Air dew point temperature $\left(T_{D P, \text { set }}\right)$

2. Air inlet temperature $\left(T_{a i r, s e t}\right)$

3. Hydrogen pressure at the FC inlet $\left(p_{\mathrm{H}_{2}}\right)$

User Interface A user interface has been established (see Figure 4.12) that allows to observe all system variables along with modifying any operating conditions. The interface is divided into two panes - the left one is for user interaction while the right one displays the state of the test bench. 


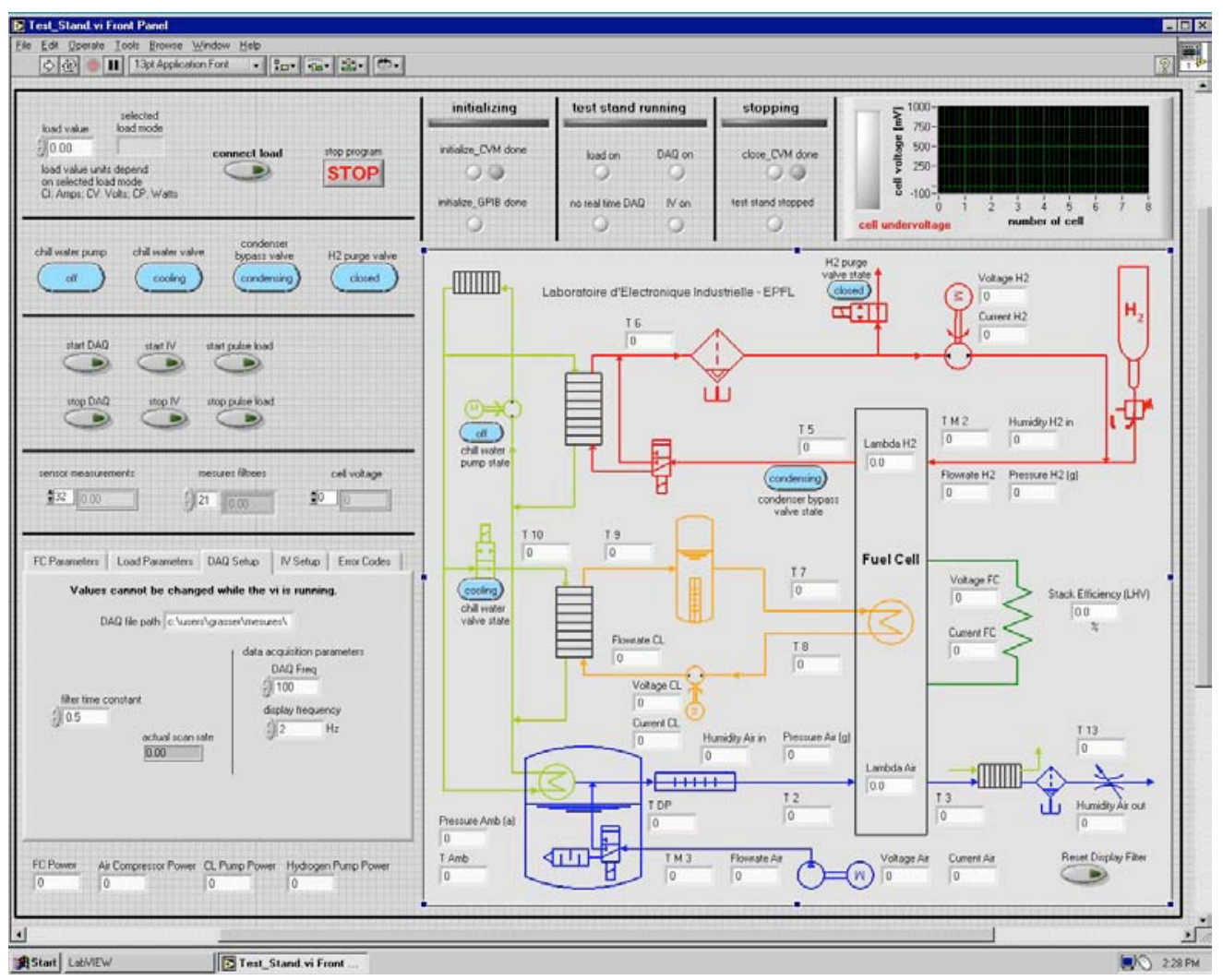

Figure 4.12: User interface for the data acquisition and control system.

The data display region shows all the acquired system variables as described on page 116. Additionally, the state of the test bench is indicated - it can be either initialising, running or stopping. For each stage, green lights signal what is currently being executed while red lights signal a problem.

The user interaction region allows to set the operating conditions for the test bench, to pilot the solenoid valves and to initiate data acquisition, pulse load or IV curve mode.

\subsection{Summary}

This chapter has presented the experimental test facilities installed at $L E I$. In the first part, the installed fuel cell stack (a joint development between $P S I$ and ETHZ) was presented. 
The characteristics of the balance of plant components of the test bench were described along with the design criteria that led to their choice. In a later part, the sensors that form the basis of the data acquisition system were introduced. Finally, the functions assumed by the data acquisition and control software were outlined.

The experimental results obtained on the test bench will be presented in chapter 5 along with a comparison with data obtained from the models developed in chapters 2 and 3. 



\section{Experimental Results}




\subsection{Introduction}

This chapter relates the fuel cell stack and balance of plant models to experimental results obtained on the fuel cell test bench installed at $L E I$.

In a first section, modelling parameters are identified for the fuel cell stack model presented in chapter 2 - both based on literature data and on local measurements. FC stack performance is then compared to the data predicted by the stack model.

Focus is then shifted to the balance of plant models presented in chapter 3 , where for each balance of plant subsystem - i.e. the air supply, the hydrogen supply and the thermal management subsystem - the model parameters are identified based on experimental data. The model performance is then evaluated and analysed.

\subsection{Fuel Cell Stack}

The analytical, cell averaged FC stack model presented in chapter 2 describes the mean cell voltage as a function of the fuel cell's operating conditions. The model is adjusted to a given fuel cell stack through a number of parameters as shown in Figure 5.1.

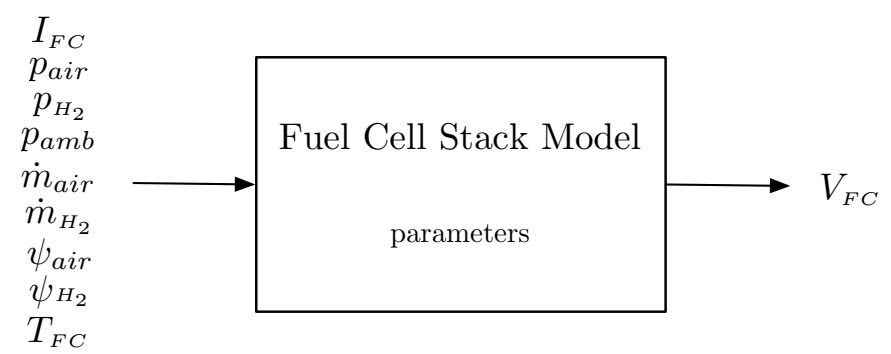

Figure 5.1: Schematic representation of the fuel cell stack model.

This chapter aims to fit the characteristic fuel cell stack parameters for the stack installed at LEI. Unfortunately, a significant degradation of the fuel cell stack was observed over time as can be seen in Figure 5.2. The stack was initially assembled in Spring 2003 from elements manufactured for the $V W$ fuel cell car developed at PSI and ETHZ.

As can be seen from the relative current-voltage curves, the degradation seems to affect different reaction mechanisms. 


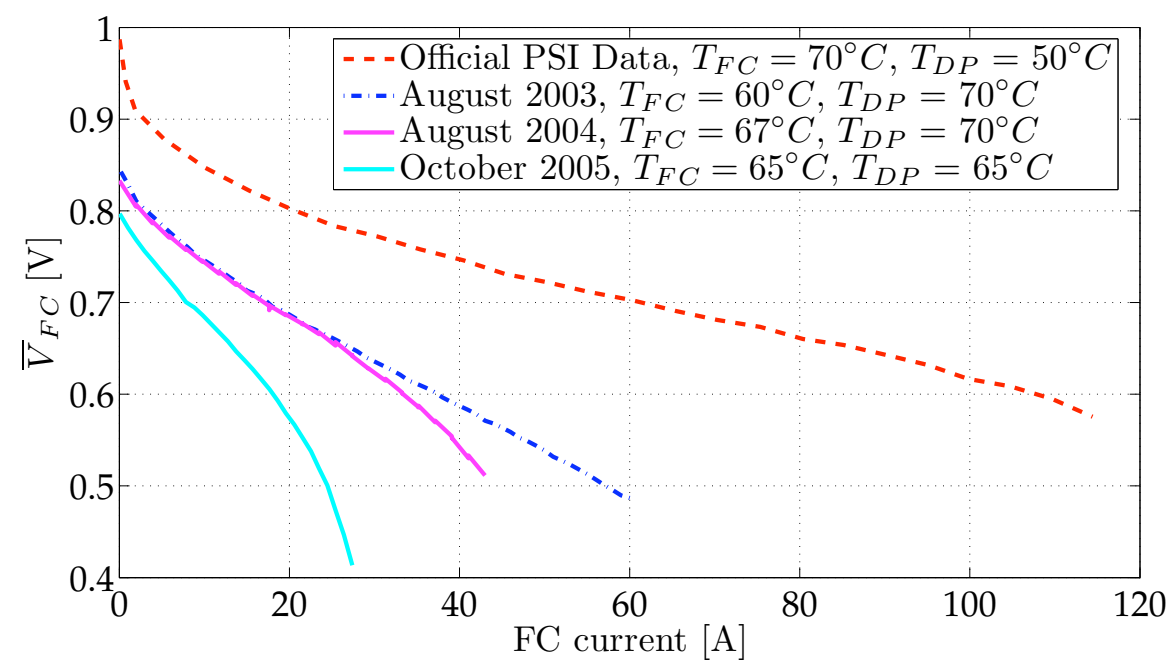

Figure 5.2: Performance evolution of the BRESA fuel cell stack installed at $L E I$. For reference purposes, the current-voltage characteristic [56] of a new stack running under design operating conditions is also shown. Note that the reference system is operated at a pressure of $2 \operatorname{bar}(a)$ whereas the $L E I$ system runs at ambient pressure.

- A degradation of the catalyst layer performance [61] is seen in the drastically lowered open circuit and low current density voltage. This can be combined with a locally thinned or pinholed membrane that allows some hydrogen crossover which decreases the equilibrium voltage.

The degradation of the catalyst layer performance is typically attributed to a chemical degradation of the catalyst particles, a decrease in active catalyst surface at the three-phase boundary due to a migration and sintering of platinum particles and a decrease of ionically conductive material at the three-phase boundary.

- A degradation of the membrane is seen in the increase of the overall resistivity. Since ohmic resistance is unlikely to vary in time, this can be attributed to a degradation of the ion transport properties of the membrane [62] [63].

- A degradation of the transport properties of the gas diffusion layer is seen through the steep drop of the IV curve at high current densities. 
This is generally attributed to a modification of the gas diffusion layer hydrophobicity [64] along with a mechanical degradation.

Additionally, as observed with many fuel cell stacks, substantial differences exist between different cells in the stack installed at LEI. This concerns both overall cell performance as well as a sensitivity to different operating conditions. Particularly, cell number 7 has very low performance along with an inability to run at high hydrogen stoichiometric flow rates and/or low air stoichiometric flow rates. Cell number 1 is unable to deliver adequate performance at higher current densities. The remaining cells (i.e. 2-6) are quite uniform in their behaviour.

This non-uniformity makes it very difficult to run the stack over wide ranges of operating conditions because any of the differing elements becomes a limiting factor.

The developed model assumes an averaged cell to describe the overall stack performance. While it provides a good approximation for a healthy fuel cell stack, an old, degrading stack's performance is less likely to be appropriately modelled. Therefore, in the context of this work, the average voltage from cells 2-6 was used as a basis for comparison with modelled data.

Because of the strong degradation observed on the experimental setup, the model has also been verified against literature data. This is outlined in chapter 5.2.1. Chapter 5.2.2 and 5.2.3 then focuses on parameter identification and model validation based on experimental data obtained from the test bench.

\subsubsection{Model Validation on Literature Data}

Literature data from Büchi et al. [56] was used to validate the fuel cell stack model developed in chapter 2. The data consists in two current-voltage curves recorded at a cell temperature of $T_{F C}=70^{\circ} \mathrm{C}$ and stoichiometric flow rates of $\lambda_{\text {air }}=\lambda_{H_{2}}=2$ with reaction gas dew point temperatures of $T_{D P}=50^{\circ} \mathrm{C}$ and $T_{D P}=65^{\circ} \mathrm{C}$ respectively.

The data set with a reaction gas dew point temperature of $T_{D P}=50^{\circ} \mathrm{C}$ was used to identify the model parameters.

Figure 5.3 shows modelled fuel cell performance against experimental data. The corresponding water transfer coefficient $\alpha$ and partial reactant pressures within the system are represented on Figure 5.4. 


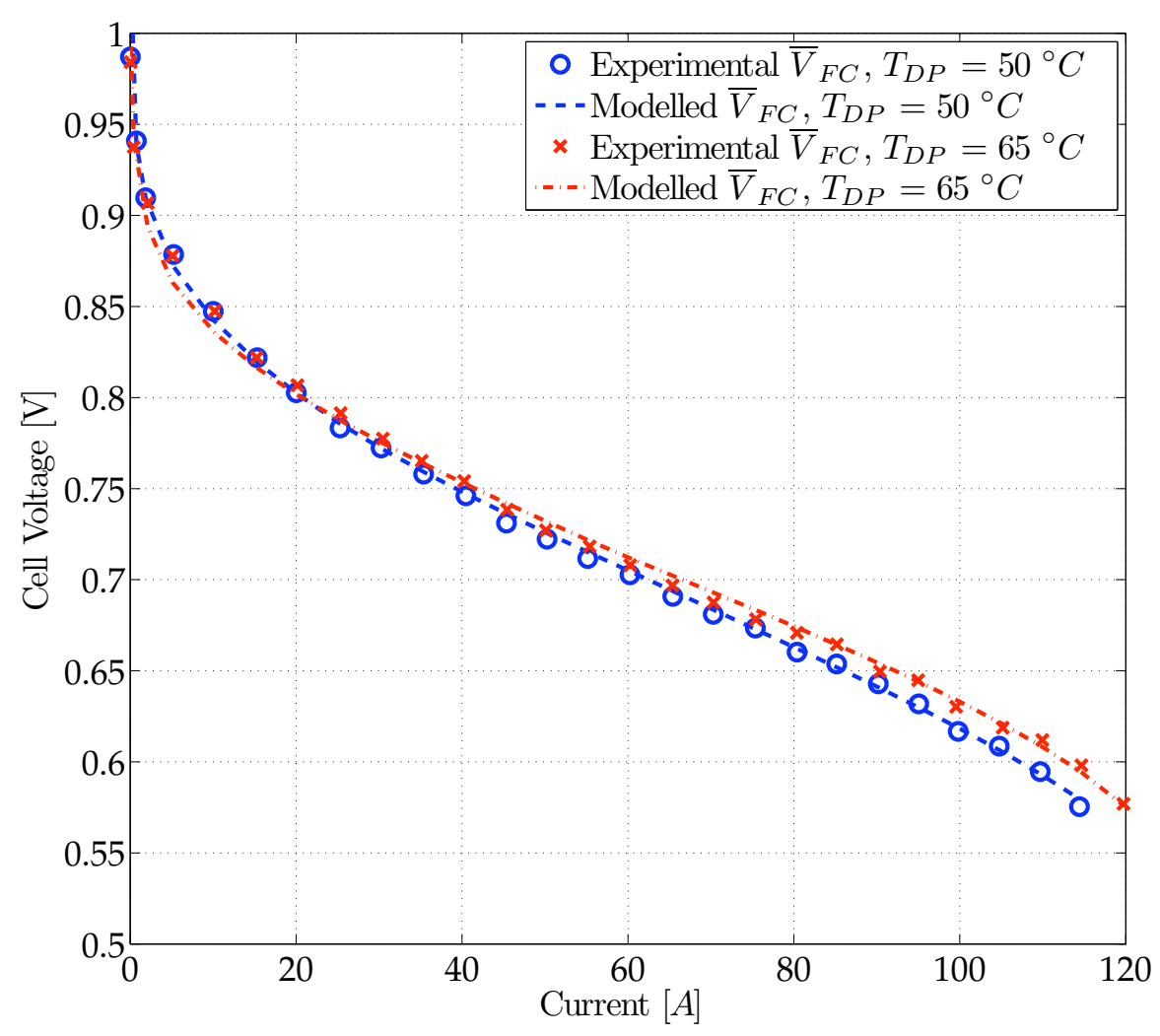

(a) Modelled and measured fuel cell voltage

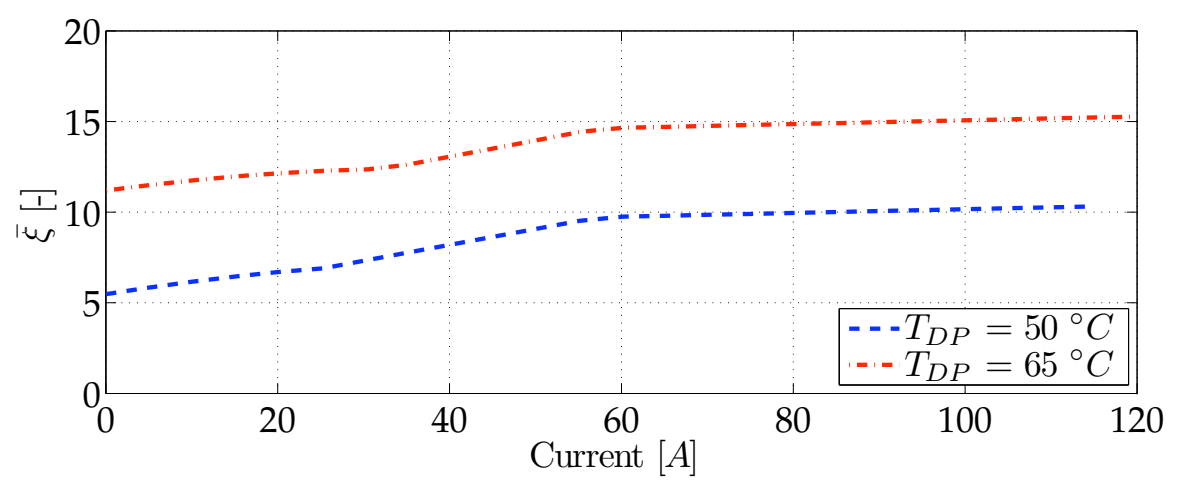

(b) Modelled average membrane water content $\bar{\xi}$

Figure 5.3: Comparison between experimental and modelled fuel cell voltage. The experimental data [56] was recorded at an average cell temperature of $T_{F C}=70^{\circ} \mathrm{C}$, stoichiometric flow rates of $\lambda_{a i r}=\lambda_{H_{2}}=2$ and dew point temperatures of $T_{D P}=50^{\circ} \mathrm{C}$ and $T_{D P}=65^{\circ} \mathrm{C}$ respectively. 


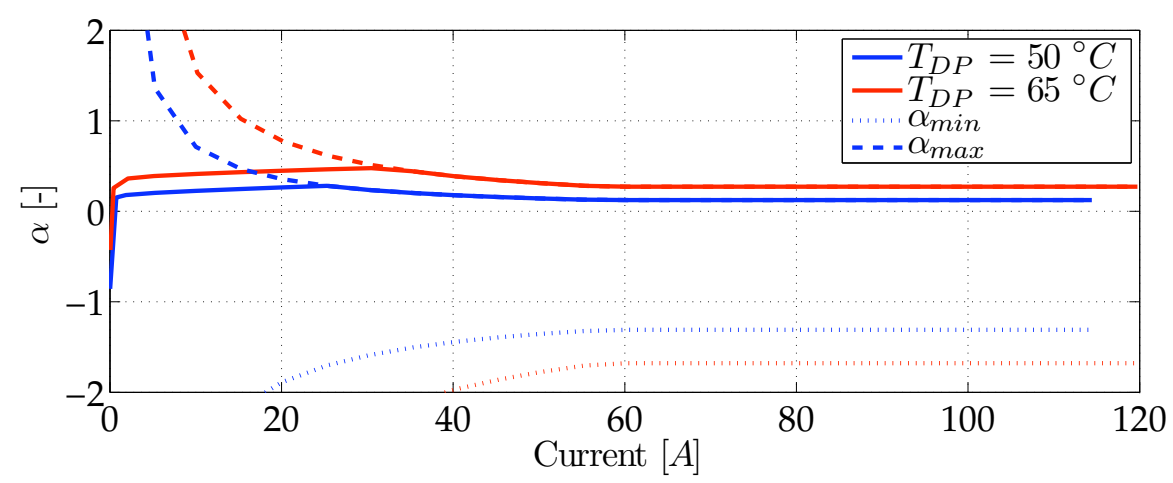

(a) Modelled water transfer coefficient $\alpha$

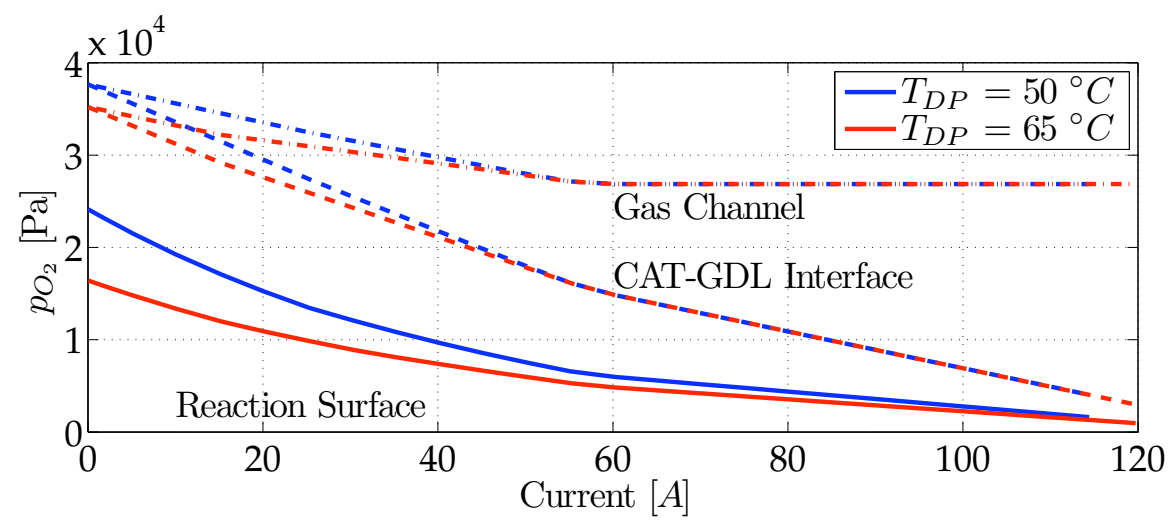

(b) Modelled partial oxygen pressures $p_{O_{2}}$

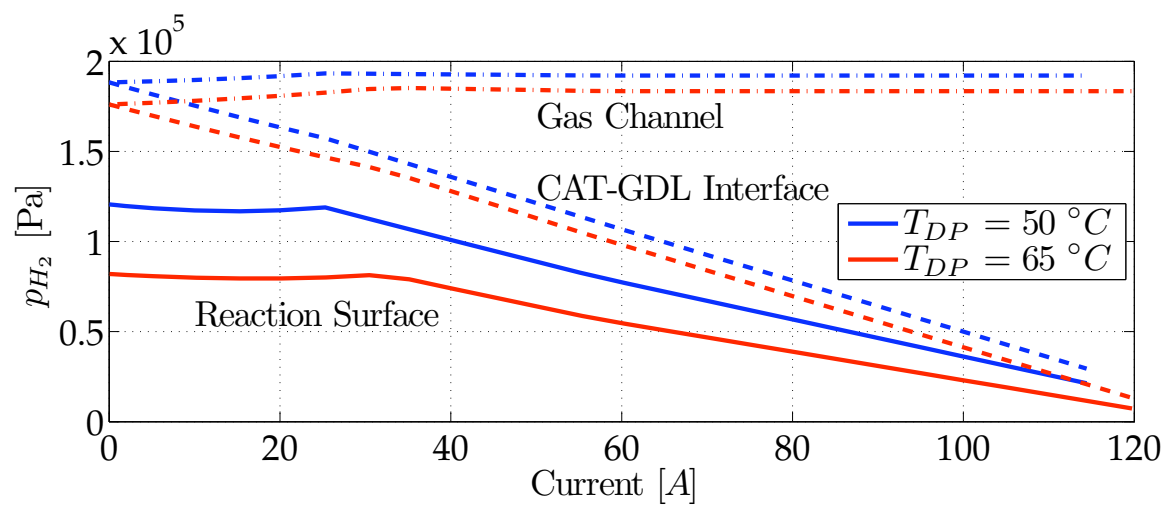

(c) Modelled partial Hydrogen pressures $p_{H_{2}}$

Figure 5.4: Modelled water transfer coefficient $\alpha$ and partial reactant pressures for the performance data shown in Figure 5.3. 
The respective model parameters can be found in Table 5.2. Parameters that were considered constant (i.e. a function of a particular FC stack's design) are listed in Appendix E.3.

Since no experimental data was available to do a fitting of the parameters relating to water transport - namely $\gamma, d_{1}$ and $k_{p}$ - literature values were used for those parameters. Note that changing the value of $k_{p}$ does not affect the model output because the anode and cathode pressures were equal during the experiment.

The gas diffusion layer permeability $\gamma$ mainly affects the membrane water content for a given set of operating conditions. During the fitting procedure, $\gamma$ was adjusted slightly from its literature value to yield the membrane water contents shown in Figure 5.3(b).

The resulting water transfer coefficient $\alpha$ shown in Figure 5.4(a) is positive for most of the IV curve indicating a net water flow from the anode to the cathode compartment. For current values larger than roughly $30 \mathrm{~A}$, the modelled water transfer coefficient corresponds to the maximum value admissible from mass balance considerations. It suggests a dominance of electro-osmotic drag as a transport mechanism within the membrane and is a strong indication that the literature values for the water transport parameters need to be further adapted to correctly describe the overall water transport. Further details on this procedure will be given in chapter 5.2.2 where appropriate experimental data was collected.

The average partial oxygen pressure in the gas channel depicted in Figure 5.4(b) decreases with increasing current since the partial water pressure increases due to water production. Beyond a current of roughly $60 A$, the saturation limit is reached. The partial oxygen fraction remains constant and additional water condenses. The same graph shows the oxygen partial pressure after diffusion through the GDL (denoted as CAT-GDL Interface) along with its value at the reaction site after dissolution in the Nafion-Water mixture composing the three phase boundary.

The partial pressure evolution for hydrogen is shown in Figure 5.4(c). The partial hydrogen pressure in the gas channel increases up to a current of roughly $30 \mathrm{~A}$. Beyond that value, it remains constant since all water entering the anode is transported across the membrane. This is a direct consequence of the water transfer coefficient $\alpha$ tending to its maximum value (See Figure 5.4(a)).

The modelled fuel cell voltage shown in Figure 5.3(a) was then fitted to the experimental data by adjusting the oxygen and hydrogen diffusivit- 
ies ${ }^{1}$ through $k_{O_{2}, w}$ and $k_{H_{2}, w}$, the exchange current density ${ }^{2}$ through $\zeta$, the equivalent ohmic resistance $R_{e q}$ and the ionic conductivity ${ }^{3}$ through $k_{\sigma}$.

With the thus fitted parameters, the model is able to predict the change in cell performance due to a change in inlet gas humidity. The prediction error remains inferior to $2 \%$ over the entire operating range.

\subsubsection{Fuel Cell Parameter Identification}

Experimental data collected on the LEI test bench was used to determine the model parameters for the installed FC stack. This was carried out in two distinct stages.

In a first stage, water collected during steady state operation of the fuel cell was used to determine any water transport relevant parameters. In a second stage, pseudo steady state data (i.e. IV curves) are exploited to fit the remaining performance related modelling parameters.

The same dependence matrix as shown in chapter 2.6 for the operating conditions has been established for the different modelling parameters that may be adjusted to fit the model to a given fuel cell stack. It is shown in Table 5.1.

\section{Water Transport Relevant Parameter Identification}

In order to identify the water transport relevant parameters (i.e. $\gamma, d_{1}$ and $k_{p}$ ), the fuel cell was run for extended periods of time at a given operating point. During that time, liquid water was collected from the water separators in the anode and cathode compartments (see Figure 4.3). This allows to determine the average water transfer coefficient for those operating conditions with a degree of redundancy.

It was found that large discrepancies exist between the amounts collected on the anode and cathode side. This could be traced to a faulty dew point temperature read-out from the humidification system. It remains yet to be shown whether this is due to a malfunctioning sensor or actuator. Through the amounts of liquid water collected, the air dew point temperature error could be evaluated at $-12.3{ }^{\circ} \mathrm{C}$ with a standard deviation of $2{ }^{\circ} \mathrm{C}$. The

\footnotetext{
${ }^{1}$ See Appendix B.4.1 for more details on how $k_{O_{2}, w}$ and $k_{H_{2}}$,w affect the respective diffusivities.

${ }^{2}$ See chapter 2.3.1 for more details on how $\zeta$ affects the exchange current density.

${ }^{3}$ See chapter 2.3.4 for more details on how $k_{\sigma}$ affects the ionic conductivity.
} 


\begin{tabular}{cccccccccc}
\hline Variable & $\mathrm{f}(?)$ & $k_{\mathrm{O}_{2}, w}$ & $k_{H_{2}, w}$ & $\gamma$ & $\zeta$ & $R_{e q}$ & $d_{1}$ & $k_{p}$ & $k_{\sigma}$ \\
\hline$\alpha$ & & & & $\bullet$ & & $\bullet$ & $\bullet$ & \\
$\bar{\chi}_{i}$ & $\alpha$ & & & $\circ$ & & $\circ$ & $\circ$ & \\
$\bar{\xi}$ & $\alpha, \bar{\chi}_{i}$ & & & $\bullet$ & & $\bullet$ & $\bullet$ & \\
$p_{O_{2}, S}$ & $\alpha, \bar{\chi}_{i}$ & $\bullet$ & & $\circ$ & & $\circ$ & $\circ$ & \\
$p_{H_{2}, S}$ & $\alpha, \bar{\chi}_{i}$ & & $\bullet$ & $\circ$ & & $\circ$ & $\circ$ & \\
$\eta_{\text {conc }}$ & $p_{i, S}$ & $\circ$ & $\circ$ & $\circ$ & & $\circ$ & $\circ$ & \\
$\eta_{\text {act }}$ & $p_{i, S}$ & $\circ$ & $\circ$ & $\circ$ & $\bullet$ & & $\circ$ & $\circ$ & \\
$\eta_{\text {ohm }}$ & $\bar{\xi}$ & & & & & $\bullet$ & & & \\
$\eta_{\text {ion }}$ & $\bar{\xi}$ & & & $\circ$ & & & $\circ$ & $\circ$ & $\bullet$ \\
$V_{\text {tot }}$ & & $\circ$ & $\circ$ & $\circ$ & $\circ$ & $\circ$ & $\circ$ & $\circ$ & $\circ$ \\
\hline
\end{tabular}

Table 5.1: Parameter dependency matrix for the fuel cell stack model. - denotes a direct dependence of the variable on the considered parameter. - denotes an indirect dependence where the variable depends on another, previously calculated variable that is itself dependent on the considered parameter.

experimental dew point temperatures in this chapter have therefore been corrected by that value.

Unfortunately, the pronounced, non-uniform degradation of the stack made it very difficult to carry out those experiments over wide ranges of operating conditions. Non-realistic (i.e. for stand-alone applications) stoichiometric flow rates were required on the cathode side to get any appreciable current densities from the stack. On the hydrogen side, dry-out was encountered for stoichiometric flow rates above 4 and flow rates below 3 were not doable because of the reduced stack current compared to the design point of the test bench.

Figure 5.5(a) shows the experimental and modelled water transfer coefficient $\alpha$ while Figure 5.5(b) and (c) show the corresponding operating conditions.

Note that while the abscissa represents the time in minutes, the data has been concatenated from different measurements. Transients between the different operating conditions have been omitted.

Up to around $330 \mathrm{~min}$, hydrogen exiting from the fuel cell stack was re-circulated through the condenser thus maintaining a stack inlet dew point around $28^{\circ} \mathrm{C}$. For the remaining data, the condenser was bypassed 


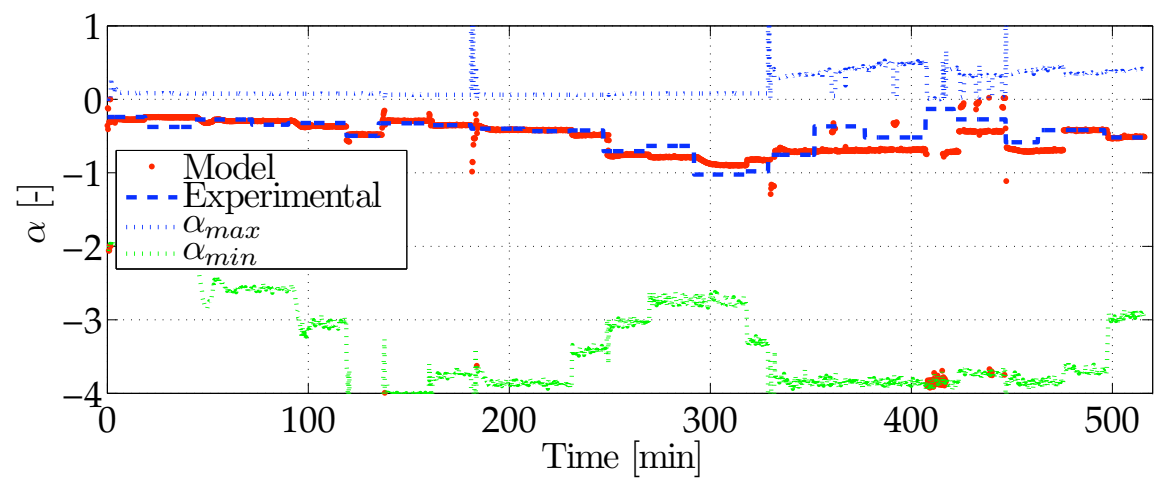

(a) Experimental and modelled water transfer coefficient $\alpha$

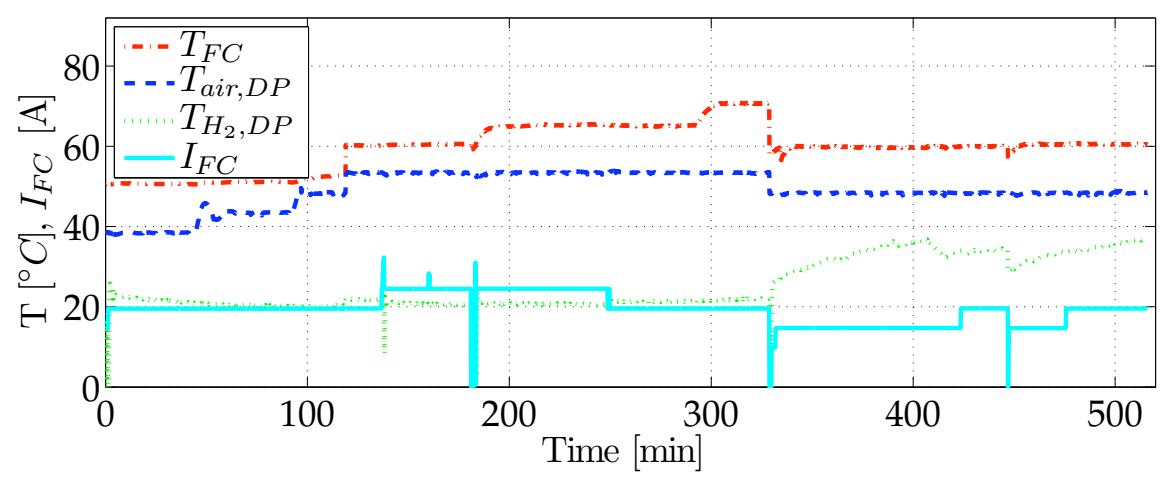

(b) Experimental gas humidities, FC temperature and FC current

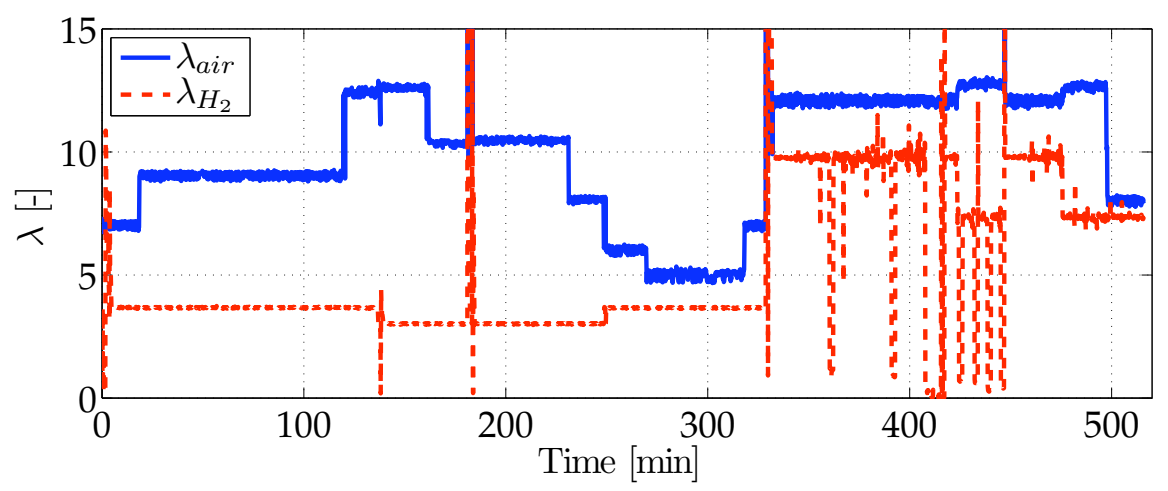

(c) Experimental stoichiometric flow rates

Figure 5.5: Experimental and modelled water transfer coefficients $\alpha$ used for identification of the water transport relevant model parameters. 
to get more humid hydrogen to the anode compartment. The resulting high humidity led to condensation in the gas stream which caused frequent malfunctioning of the humidity and flow meter with its integrated temperature sensor. This can be observed on the erratic measurements for $\lambda_{H_{2}}$ in Figure 5.5(c).

The values identified for $\gamma, d_{1}$ and $k_{p}$ are shown in Table 5.2. For $\gamma$ and $k_{p}$, reasonable agreement with literature data is found. The identified value for $d_{1}$ is - depending on the data source - between two and three orders of magnitude larger than the values found in literature. This is believed to be due to two reasons:

- An overestimation of the effective water transfer through electroosmotic drag by the current model formulation.

- The assumption of a linear water sorption characteristic of the membrane phase. This is one of the major assumptions that allow an analytical description of overall water transport. At the same time, it is a strong simplification of experimental data (see Figure 2.2) where a steep gradient is observed in the vicinity of a water activity $a=1$.

The modelled water transfer coefficient $\alpha$ shows very good agreement with experimental data in the part where the stack was run on dry hydrogen. When running on humid hydrogen, the prediction error is larger. It cannot be ascertained at this point whether this is due to badly identified parameters, modelling problems or simply to faulty humidity and temperature readouts.

\section{Cell Voltage Relevant Parameter Identification}

The remaining modelling parameters describing reactant gas transport (i.e $k_{O_{2}, w}, k_{H_{2}, w}$ and $H_{0}$ ), activation overpotential (i.e. $\zeta$ ), ohmic overpotential (i.e. $R_{e q}$ ) and ionic overpotential (i.e. $k_{\sigma}$ ) were curve fitted based on data shown in Figure 5.6(a) to Figure 5.6(b). Their respective values can be found in Table 5.2.

The reduced values of $k_{O_{2}, w}$ and $k_{H_{2}, w}$ with respect to the ones identified from official PSI data (see chapter 5.2.1) are consistent with the observed decrease in maximum current density of the fuel cell stack.

A modification in the gas diffusion layer properties can also be seen from the increase in pressure drop across the cathode compartment (see Figure 5.10) with respect to design data. 


\begin{tabular}{lcccc}
\hline Parameter & Units & Literature Value & PSI Stack & LEI Stack \\
\hline$H_{0}$ & $\frac{P a \cdot m^{3}}{m o l}$ & 4000 & 4000 & 4000 \\
$k_{O_{2}, w}$ & - & $3.5 \cdot 10^{-7}$ & $9 \cdot 10^{-8}$ & $1.7 \cdot 10^{-8}$ \\
$k_{H_{2}, w}$ & - & $2.1 \cdot 10^{-6}$ & $2.3 \cdot 10^{-8}$ & $6.1 \cdot 10^{-9}$ \\
$\gamma$ & $\frac{m}{s}$ & $5.6 \cdot 10^{-6}$ & $7 \cdot 10^{-6}$ & $2 \cdot 10^{-5}$ \\
$\zeta$ & - & $\epsilon[33.938 .4]$ & 36.20 & 35.15 \\
$R_{e q}$ & $\Omega$ & $\epsilon[2.255 .4] \cdot 10^{-4}$ & $4.4 \cdot 10^{-4}$ & $6 \cdot 10^{-4}$ \\
$d_{1}$ & $\frac{m^{2}}{s}$ & $4.96 \cdot 10^{-7}$ & $5 \cdot 10^{-7}$ & $2.2 \cdot 10^{-4}$ \\
$k_{p}$ & $\frac{m^{2}}{s}$ & $1.8 \cdot 10^{-18}$ & $1.8 \cdot 10^{-18}$ & $3 \cdot 10^{-19}$ \\
$k_{\sigma}$ & $\frac{S}{m}$ & 0.51 & 0.24 & 0.06 \\
\hline
\end{tabular}

Table 5.2: Literature fuel cell stack model parameters compared to values identified for experimental data from Büchi et al. [56] and experimental data from the $L E I$ test bench.

The increase in activation overpotential is reflected by a decrease of $\zeta$. The equivalent electronic resistance $R_{e q}$ is mainly composed of the resistance of the GDL media which depends strongly on the clamping pressure applied to stack. Accounting for a reduced clamping force of the $L E I$ stack with respect to the ones assembled at PSI, the equivalent resistance $R_{e q}$ was increased slightly. This is merely an educated guess since - with the limited data available - an increase in $R_{e q}$ can be compensated by increasing the ionic conductivity $k_{\sigma}$ to obtain a very similar IV curve.

Finally, the dramatic reduction in ionic conductivity with respect to literature data (see chapter 5.2) is represented in the decrease of $k_{\sigma}$.

\subsubsection{Fuel Cell Stack Model Validation}

The model was then run with the identified parameters on the experimental data shown in Figure 5.6(c) to (h). All IV curves were recorded at different temperatures, humidities and stoichiometric flow rates. To illustrate the disproportional degradation of cell 7 , its voltage is shown as a dotted line on the respective IV curves.

Note that for all data, the model does not correctly describe the behaviour at low current densities. This is the region where the activation overpotential dominates. It is believed that the degradation of the fuel cell 

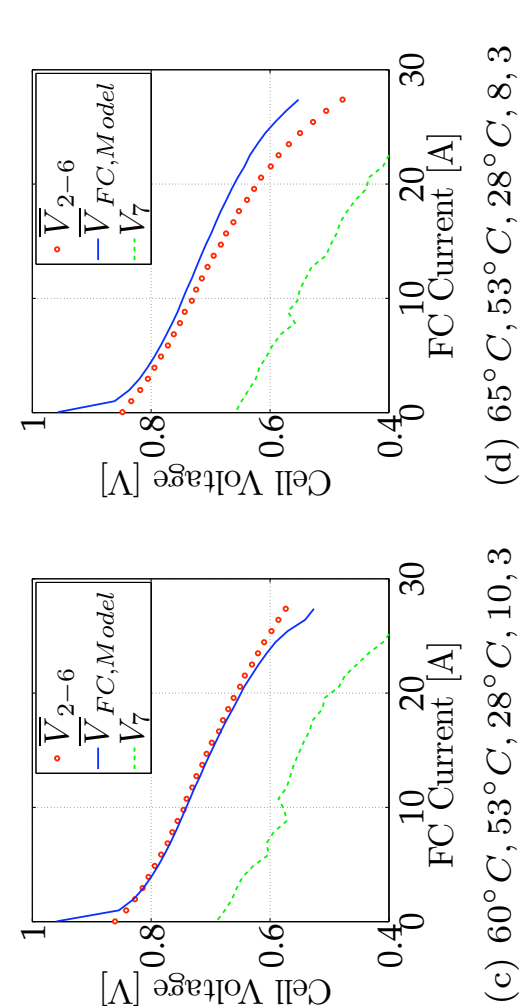

$[\Lambda]$ ә.вет[о $\Lambda$ ІІәО
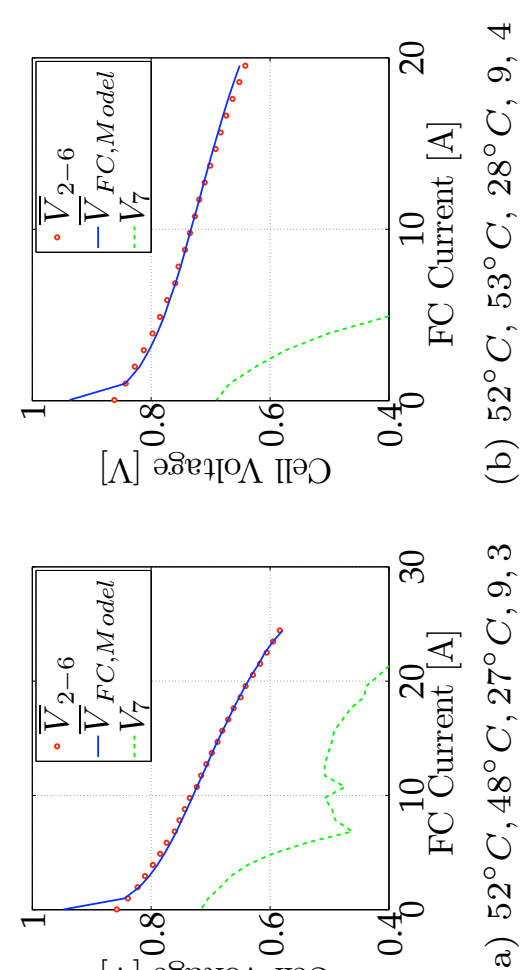

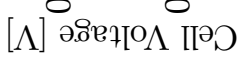
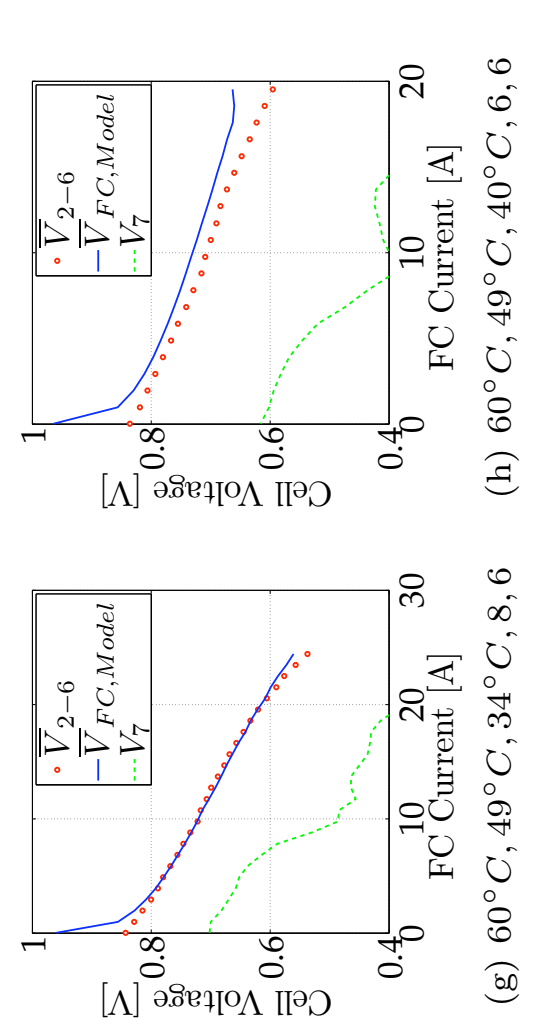

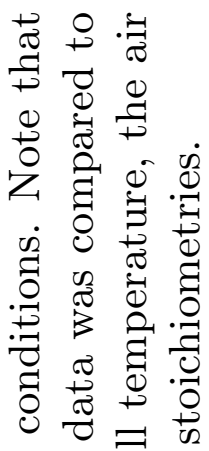

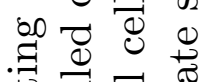

击

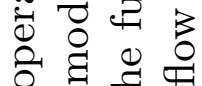

๖ 0 E

.

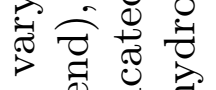

-

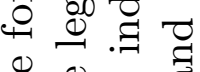

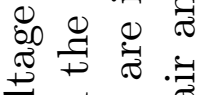

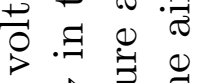

$\Rightarrow N$ 万人

0 च

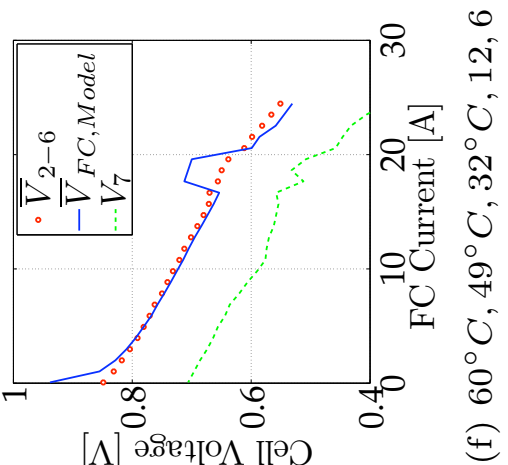

ฮిం

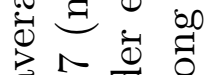

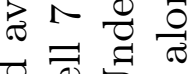

ठ

苟

리

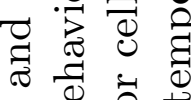

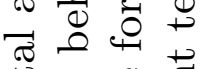

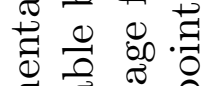

घี

氙焉

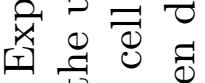

$\because \Psi 08$

అ艹

$\begin{array}{llll}10 & 0 & 0 & 0 \\ 0 & 0 & 0\end{array}$

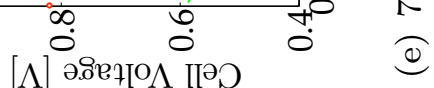

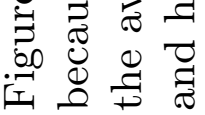


stack (see chapter 5.2) has modified the structure of the membrane-catalyst layer interface in such a way that the initial modelling assumption (i.e. considering the catalyst layer an interface where the space dependent and interlinked description of gas transport, ionic and ohmic overpotential can be neglected ${ }^{4}$ ) for the catalyst layer does not hold any more. This is most likely combined with a thinned or pin-holed membrane that leads to a lowered open circuit potential.

Good agreement between modelled and experimental data is observed for the data used for parameter identification (i.e. Figure 5.6(a) to (c)) where the fuel cell temperature and air humidity were varied.

The data shown in Figure 5.6(d) and (e) was recorded with increased fuel cell temperatures of 65 and $70^{\circ} \mathrm{C}$. At an air dew point of $53^{\circ} \mathrm{C}$ and a stoichiometric air flow rate between 7 and 8 , fuel cell dry-out occurs. The dry-out is so severe, that the modelling assumption of a well functioning three-phase boundary catalyst layer no longer holds and the model is unable to describe the experimentally observed performance.

Figures Figure 5.6(f) to (h) were recorded with various degrees of hydrogen humidification. Unfortunately (see chapter 5.2.2), this led to problems with the hydrogen mass flow, humidity and temperature. This is evident on Figure 5.6(f) where faulty hydrogen mass flow readout causes the modelled voltage to peak just below $20 \mathrm{~A}$.

Despite this, good agreement between experimental and modelled data is shown in Figure 5.6(f) and (g). The condensation related malfunctioning of the hydrogen mass flow and humidity sensors are most likely at the origin of the discrepancy between modelled and experimental data shown in Figure 5.6(h).

\subsubsection{Summary}

Excellent agreement was shown between modelled and experimental data from literature. This gives an idea of the model's performance, but cannot be generalised since not enough experimental data was available to validate the model over wide ranges of operating conditions.

As noted in chapter 5.1, the pronounced degradation of the fuel cell stack made it impossible to get data over a wide range of operating conditions. The degradation of the stack prevented the model from correctly predicting the open circuit voltage.

\footnotetext{
${ }^{4}$ See chapter 2.3 .1 for more details.
} 
Steady state measurements with water collection were carried out and allowed identification of the water transport relevant modelling parameters and detection of problems with the humidification system. Further modelling parameters were identified on a set of pseudo-steady state IV curves.

Model validation showed good agreement between experimental and modelled data while operating the stack under healthy conditions. Under dry-out conditions, initial modelling assumptions no longer hold and the model is unable to predict stack performance.

\subsection{Air Supply Subsystem}

The air supply system model described in chapter 3.3 describes the evolution of the compressor current, the compressor speed and the air pressure in the inlet manifold as a function of the applied compressor voltage and the ambient pressure.

Chapter 5.3.1 outlines how the parameters characterising the model were identified for the given experimental set-up. The obtained model is then compared to further experimental results in chapter 5.3.2.

\subsubsection{Parameter Identification}

This section outlines the techniques and data used for parameter identification of the air supply subsystem model. Since there are a substantial number of parameters to be identified, certain parameters have been identified from dedicated measurements.

\section{Identification of $R_{C O}$ and $L_{C O}$}

The electrical resistance and inductance of the air compressor were directly obtained from the manufacturer. Their respective values can be found in Table 5.3.

\section{Identification of $k_{t, C O}$}

For the torque constant $k_{t}$ of the air compressor's electric motor, steady state measurements under different compressor loads have been carried 
out. The resulting current, voltage and rotational speed measurements then allow to determine the motor's torque constant ${ }^{5}$.

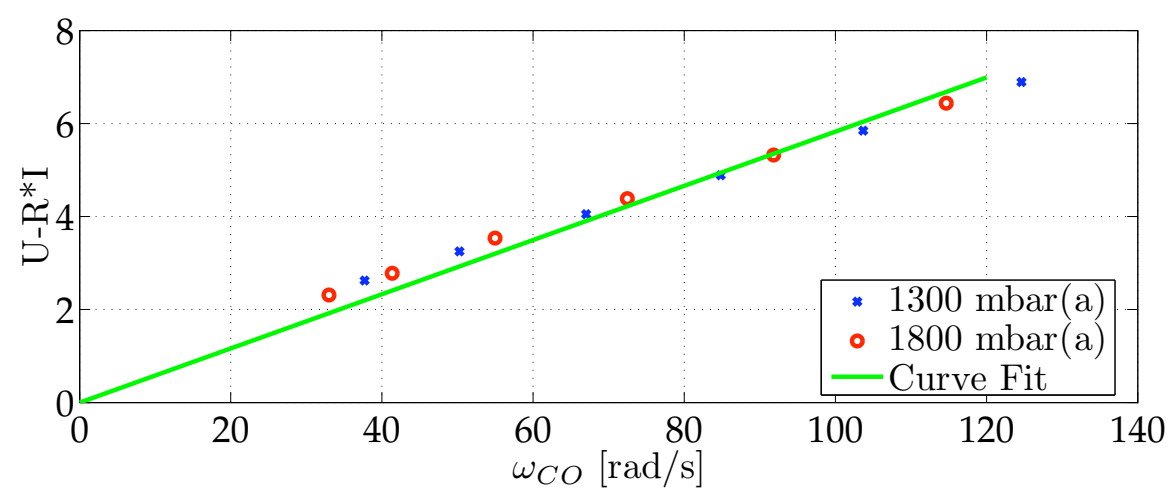

Figure 5.7: Identification of the air compressor motor's torque constant $k_{t}$.

The measurements are represented graphically in Figure 5.7. They correspond to a compressor load of roughly $30 \%$ (i.e. a compressor outlet pressure of $1.3 \operatorname{bar}(a))$ along with a second measurement series recorded at a compressor load of about $80 \%$. An overall linear interpolation is used to determine the torque constant. The resulting value of $k_{t, C O}=5.2 \cdot 10^{-2} \frac{\mathrm{N} \cdot \mathrm{m}}{\mathrm{A}}$ is roughly $20 \%$ larger than the manufacturer specification.

\section{Identification of $k_{m, C O}$}

The speed-flow rate coefficient $k_{m}$ can be identified from the same steady state measurements as the torque constant in the previous section - Figure 5.8 represents that data graphically.

Note that the data suggests a dependence of $k_{m, C O}$ on the compressor load. Neglecting this dependence and doing an overall linear interpolation yields $k_{m, C O}=0.38 \frac{\mathrm{slpm} \cdot \mathrm{s}}{\mathrm{rad}}$. This value is about $30 \%$ higher than the one obtained through a batch identification of several air supply subsystem parameters through a non-linear least squares algorithm (see Table 5.3).

\footnotetext{
${ }^{5}$ Motor voltage and current were recorded by the test bench while speed measurements were undertaken manually with a handheld device
} 


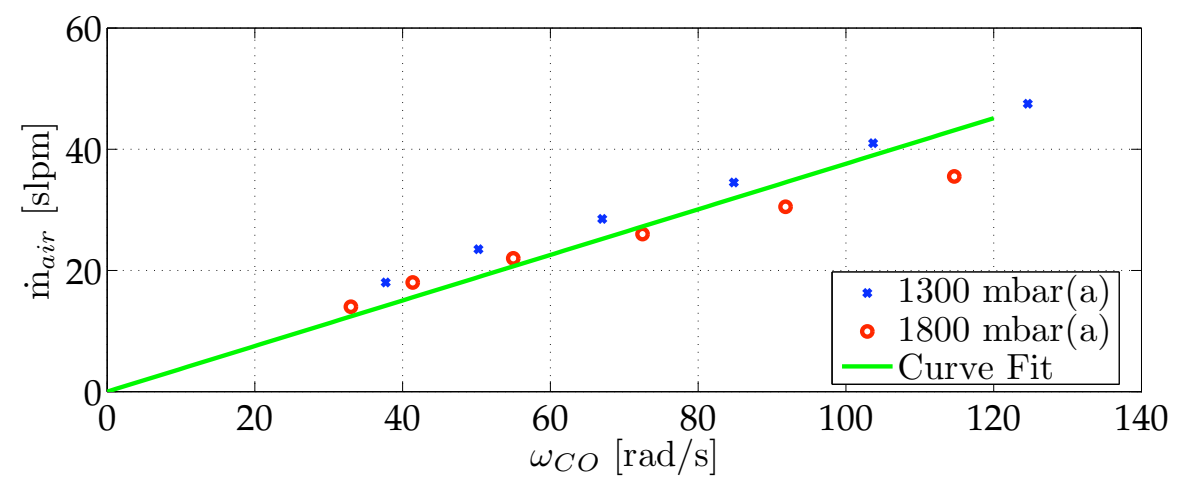

Figure 5.8: Identification of the air compressor's speed-mass flow coefficient $k_{m}$.

Identification of $J_{C O}, k_{p, C O}$ and $k_{f, C O}$

The parameters relating to the air compressor were identified on the reduced model outlined in chapter 3.3.1 where the actual air inlet pressure is considered a system input.

The experimental data used in this chapter was obtained on a set-up with an equivalent hydraulic circuit, where the fuel cell was replaced by a flow resistance consisting of an adjustable valve. This allowed verification of most parameters while minimising the risk of damaging the membrane through pressure transients without counter balancing on the anode side. This risk was heightened by the degradation of the fuel cell stack that led to a important increase in pressure drop across the cathode gas channel.

Additionally, by varying the actual flow resistance of the valve during the experiments, more modes of the system could be excited which is particularly useful in determining the pressure coefficient $k_{p, C O}$ of the air compressor.

Identification was carried out by a least squares algorithm for the prediction error on the air mass flow. The measurements and corresponding modelled data are shown on Figure 5.9, the resulting parameters are presented in Table 5.3.

Identification of $k_{h}$ and $V_{a d m}$

The actual hydraulic flow resistance of the cathode compartment was identified from steady state experimental data on the running fuel cell. 


\subsection{AIR SUPPLY SUBSYSTEM}

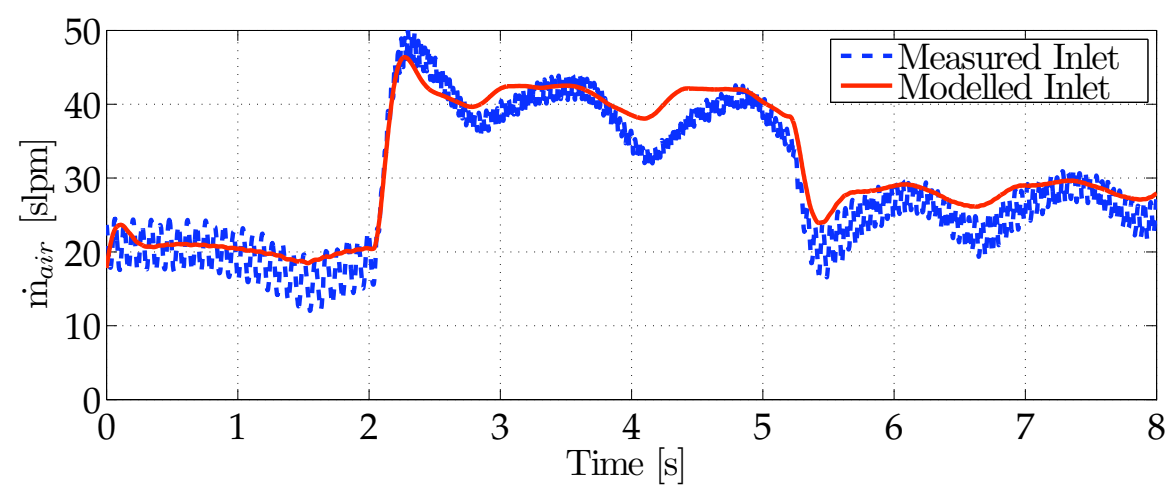

(a) Modelled and measured air mass flow at air compressor outlet

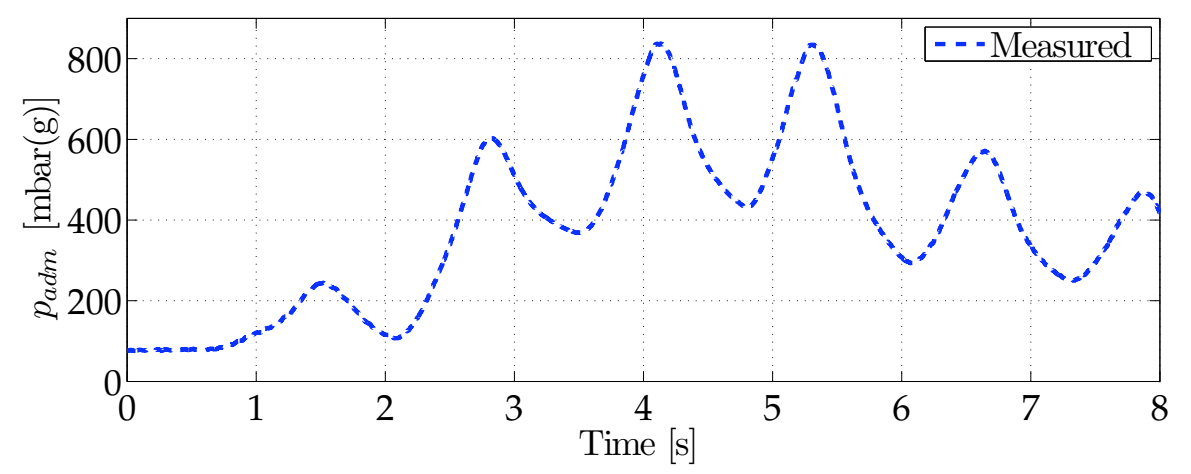

(b) Measured air pressure in the inlet manifold

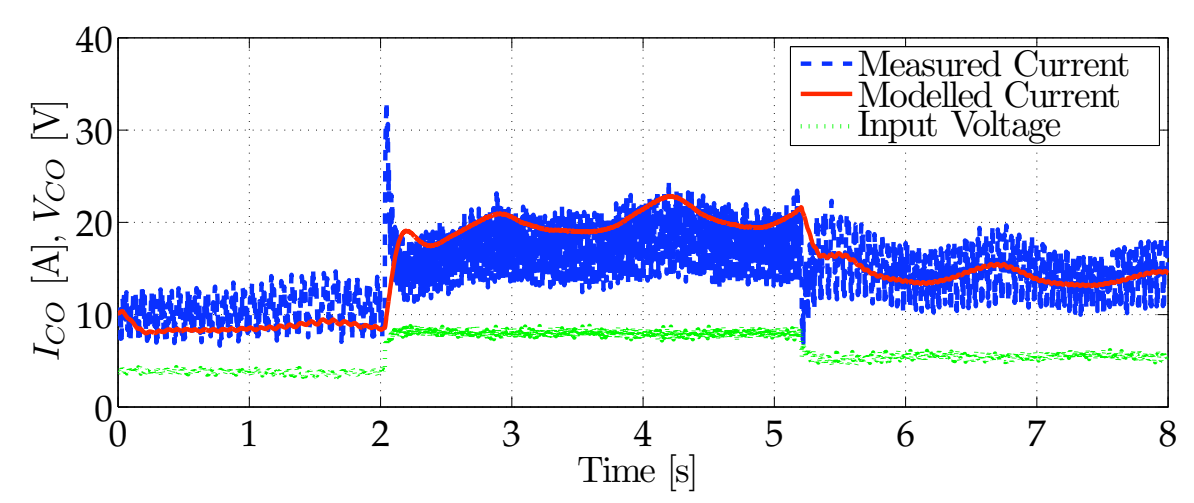

(c) Air compressor voltage and modelled and measured air compressor current

Figure 5.9: Experimental data used for parameter identification of the air supply subsystem. The fuel cell was simulated in the experimental set-up through a valve with a similar flow resistance which was varied during the experiment. The model corresponds to the one outlined in 3.3.1 where the air inlet pressure is considered a system input. 
The data is represented in Figure 5.10 along with the stack's designed pressure drop characteristic [34]. The corresponding value for $k_{h}$ is shown in Table 5.3.

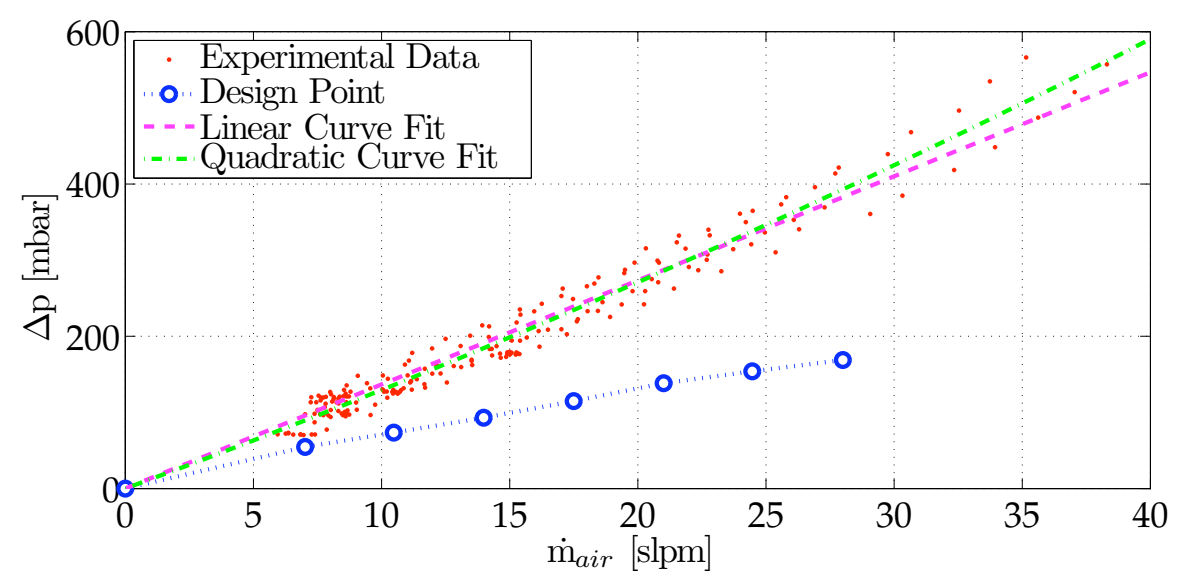

Figure 5.10: Pressure drop across the fuel cell cathode gas channel. For reference purposes, the design characteristic [34] of the stack is also shown.

Despite the observed two to two and a half-fold increase in pressure drop compared to the design point, the pressure drop remains largely linear with flow rate - indicating a laminar flow regime.

The equivalent hydraulic flow resistance of the restriction valve used in lieu of the fuel cell stack and the inlet chamber volume were obtained from measurements on the full air supply subsystem. A least squares prediction error algorithm was run on modelled and experimental pressure evolution data. The resulting value for the inlet chamber volume is presented in Table 5.3 along with the other air supply subsystem parameters.

\subsubsection{Model Validation}

The data used for model validation is shown in Figure 5.11 along with the modelled variables.

Note that, as shown in chapter 3.3.2 the product of hydraulic fuel cell resistance and inlet chamber volume determines the pressure variation time constant of the air supply manifold. This in turn represents the time constant with which pressure and mass flow changes are reflected to the fuel cell stack. 


\subsection{AIR SUPPLY SUBSYSTEM}

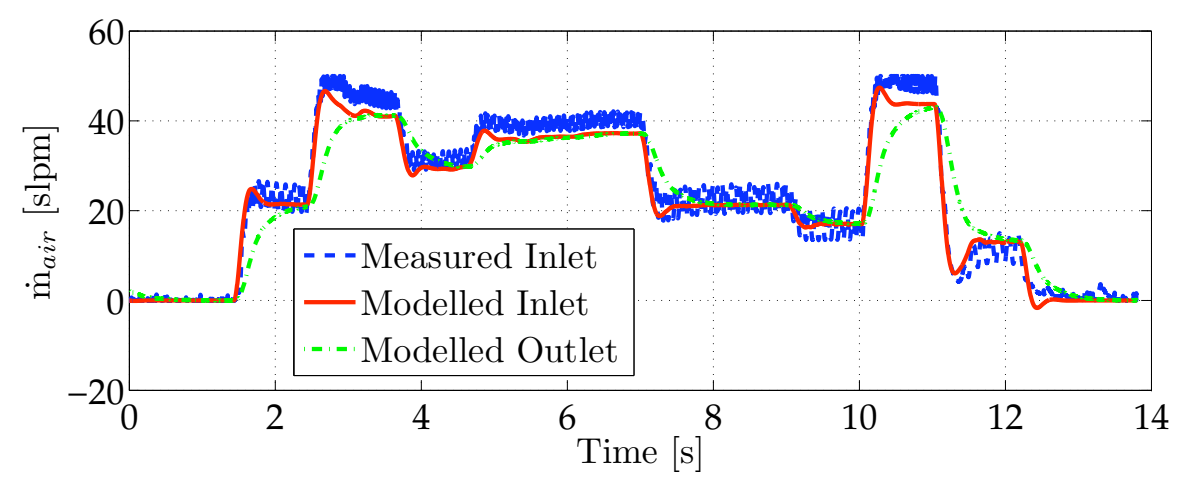

(a) Modelled and measured air mass flow at the inlet manifold inlet and modelled mass flow at the inlet manifold outlet

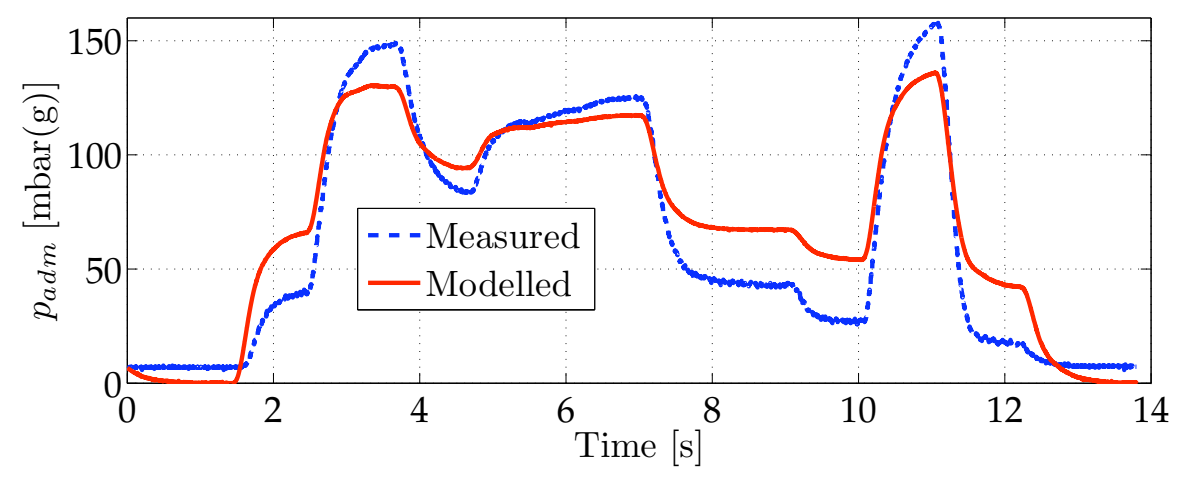

(b) Modelled and measured air pressure in the inlet manifold

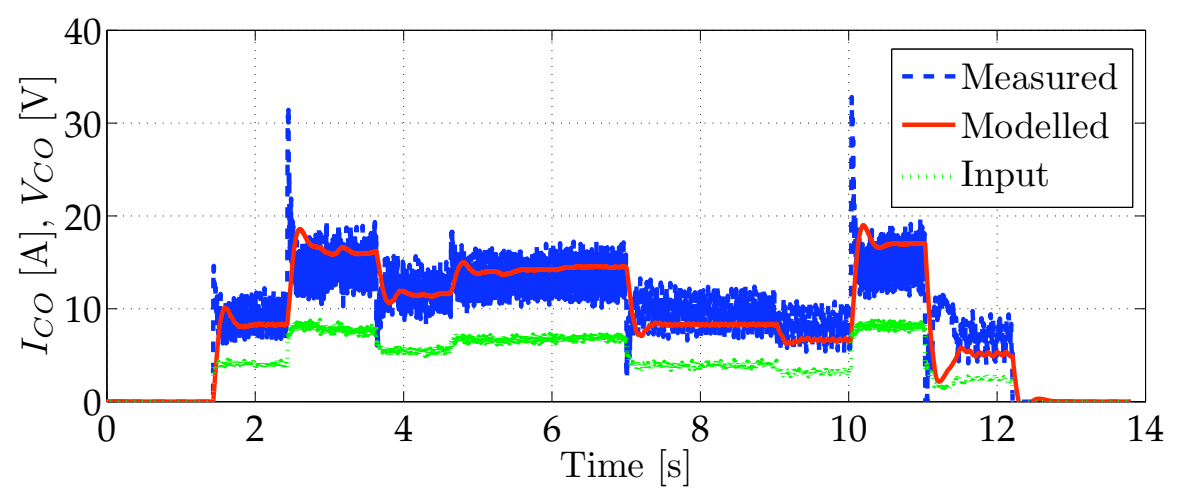

(c) Air compressor voltage and modelled and measured air compressor current

Figure 5.11: Experimental results compared to modelled data for the air supply subsystem. The fuel cell was simulated in the experimental set-up through a valve with a similar flow resistance. 


\begin{tabular}{|c|c|c|c|}
\hline Description & Parameter & Value & Units \\
\hline Motor resistance & $R_{C O}$ & 0.0818 & $\Omega$ \\
\hline Motor inductance & $L_{C O}$ & 0.0283 & $H$ \\
\hline Motor torque constant & $k_{t, C O}$ & 0.053 & $\frac{N m}{4}$ \\
\hline Equivalent inertia & $J_{C O}$ & $2.90 \cdot 10^{-4}$ & $\mathrm{~kg} \cdot \mathrm{m}^{2}$ \\
\hline Friction coefficient & $k_{f, C O}$ & $4.4 \cdot 10^{-3}$ & $N m \frac{s}{r a d}$ \\
\hline Pressure coefficient & $k_{p, C O}$ & $4.5 \cdot 10^{-6}$ & $m^{3^{a}}$ \\
\hline Speed-flow rate coefficient & $k_{m, C O}$ & 0.284 & $\frac{s l p m \cdot s}{r a d}$ \\
\hline Hydraulic FC resistance & $k_{h}$ & 1370 & $\frac{P a}{\text { slpm }}$ \\
\hline Inlet chamber volume & $V_{a d m}$ & 0.0014 & $m^{3}$ \\
\hline
\end{tabular}

Table 5.3: Experimentally identified air supply subsystem parameters.

The effect of this is not negligible, as can be seen on Figure 5.11(a) where the modelled outlet flow rate (corresponds to the air flow rate through the fuel cell stack) exhibits a time constant of around $0.3 \mathrm{~s}$. This is the main factor limiting fuel cell dynamics under changing load conditions.

The prediction error on the inlet flow rate is around $10 \%$ for flow rates above $15 \mathrm{slpm}$. For lower flow rates, the prediction error increases and is found to be around $20 \%$ for a flow rate of $10 \mathrm{slpm}$. Note the fluctuation of the measured flow rate which is a direct consequence of the use of a membrane compressor. The frequency of fluctuation is proportional to the compressor speed.

The same fluctuations are also found on the compressor current measurements where they are related to a variation in required motor torque over one cycle. The maximum average prediction errors for the motor current are in the vicinity of $20 \%$. Note the large current spikes on positive step changes in supply voltage that are not reproduced by the model.

The modelled pressure evolution correctly reproduces the time constants of the system. The actual pressure predictions however, do not follow the experimentally observed values. Upon closer inspection, this can be traced to the usage of an "equivalent" flow resistance element in lieu of the fuel cell.

While the air supply subsystem model supposes the linear flow ratepressure drop characteristics typical for laminar flow conditions, the actual flow resistance is a discharge valve. The pressure drop across such a valve 
is typically described by the nozzle equation ${ }^{6}$ which leads to a quadratic pressure drop-flow rate characteristic. This difference is exhibited by the data shown in Figure 5.11(b) where the model under-predicts the actual pressure drop at high flow rates and over-estimates the actual pressure drop at low flow rates.

The state space model corresponding to the data shown in Figure 5.11 is characterised by electro-mechanical time constants of roughly $60 \mathrm{~ms}$ and a hydraulic time constant of around $0.26 \mathrm{~s}$. The state space matrices are given by:

$$
\begin{aligned}
& {\left[\begin{array}{l}
\frac{d i_{C O}}{d t} \\
\frac{d \omega_{C O}}{d t} \\
\frac{d p_{a i r}}{d t}
\end{array}\right]=\left[\begin{array}{ccc}
-2.89 & -1.53 & 0 \\
150 & -15.2 & -0.0155 \\
0 & -8.94 \cdot 10^{6} & 5.25 \cdot 10^{4}
\end{array}\right]\left[\begin{array}{c}
i_{C O} \\
\omega_{C O} \\
p_{a i r}
\end{array}\right]} \\
& +\left[\begin{array}{cc}
35.3 & 0 \\
0 & -0.0155 \\
0 & 5.25 \cdot 10^{4}
\end{array}\right]\left[\begin{array}{c}
V_{C O} \\
p_{a m b}
\end{array}\right] \\
& {\left[\begin{array}{l}
\dot{m}_{\text {air }} \\
p_{\text {adm }}
\end{array}\right]=\left[\begin{array}{ccc}
0 & 0.284 & 0 \\
0 & 0 & 1
\end{array}\right]\left[\begin{array}{c}
i_{C O} \\
\omega_{C O} \\
p_{a i r}
\end{array}\right]}
\end{aligned}
$$

\subsubsection{Summary}

The air supply subsystem parameters have been identified based on a multitude of steady state and dynamic measurements. Because of an increased pressure drop across the fuel cell with ageing, dynamic measurements and model validation were carried out using a restriction valve in place of the fuel cell cathode compartment.

The model shows excellent performance with respect to air flow rate, particularly at higher flow rates. At flow rates below $10 \mathrm{slpm}$, prediction errors above $20 \%$ result.

The dynamic behaviour of the air supply subsystem is correctly modelled for both the air flow rate through the fuel cell and the pressure evolution in the supply manifold. Typical hydraulic time constants of around $0.3 \mathrm{~s}$ were found.

\footnotetext{
${ }^{6}$ See chapter 3.3 .5 for more details.
} 


\subsection{Hydrogen Supply Subsystem}

The hydrogen supply subsystem model described in chapter 3.4 describes the evolution of the hydrogen flow rate as a function of the applied pump current.

This chapter presents the experimental results used for parameter identification followed by a validation of the obtained model on additional experimental data.

\subsubsection{Parameter Identification}

Because of the built-in current controller, the order of the hydrogen pump model is reduced with respect to the air compressor model. This also reduces the number of parameters to be identified. This allowed direct identification of the system parameters through a non-linear least squares algorithm on experimental data.

Prior to that, the assumption of a linear pressure drop evolution with an increase in flow rate was examined based on the data shown in Figure 5.12.

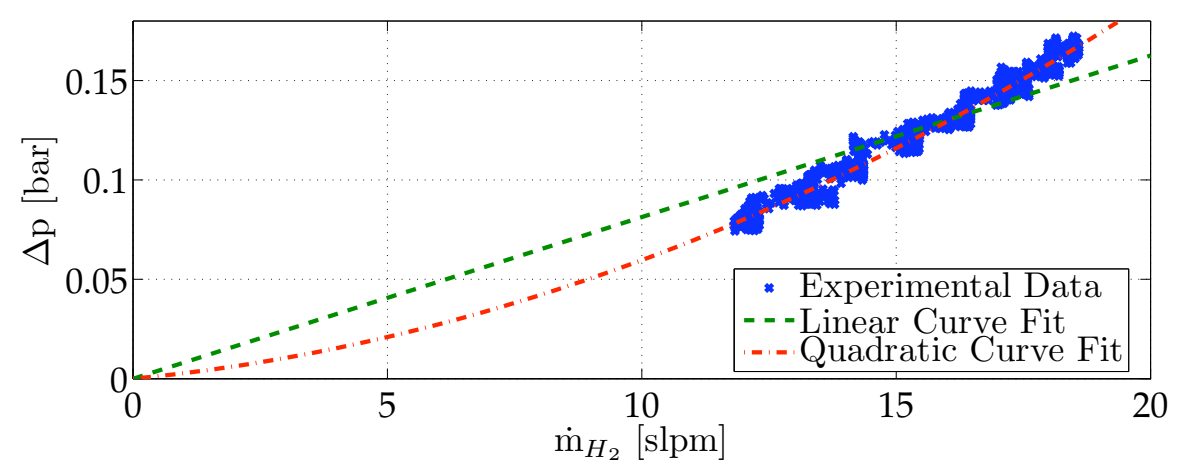

Figure 5.12: Pressure drop across the anode compartment of the fuel cell stack.

While data interpolation actually suggests a quadratic pressure drop characteristic, it can be shown from current measurements under no-load conditions that the difference between a linear and quadratic curve fit is negligible with respect to the overall friction coefficient $k_{f, H P}$.

Figure 5.13 shows the measurements used for parameter identification of the hydrogen supply subsystem. Note that as with the air compressor, the membrane pump causes a strongly oscillating flow rate. As a consequence, 
the flow rate measurements depicted in Figure 5.13 correspond to filtered experimental data.

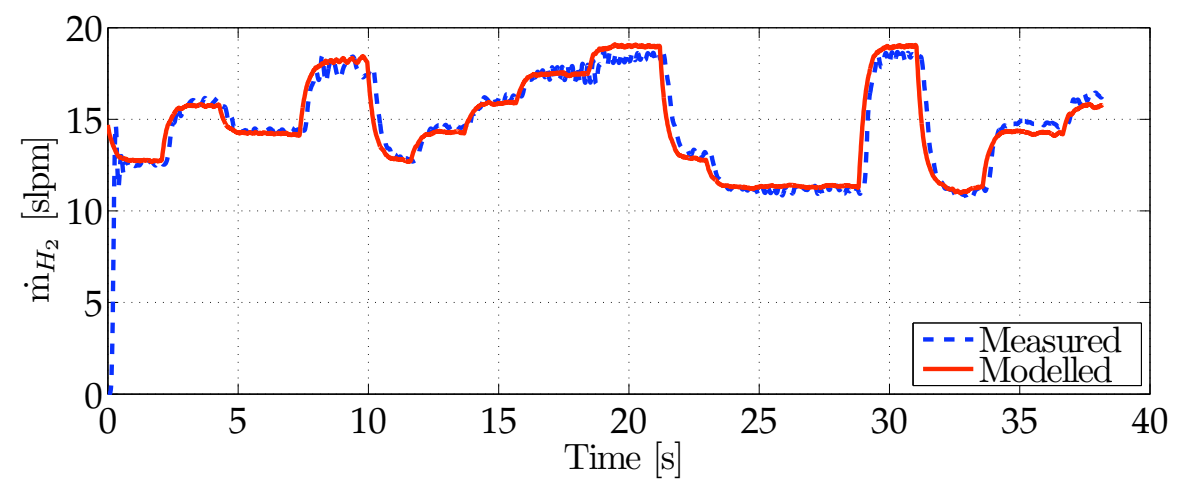

(a) Modelled and measured hydrogen mass flow through the fuel cell

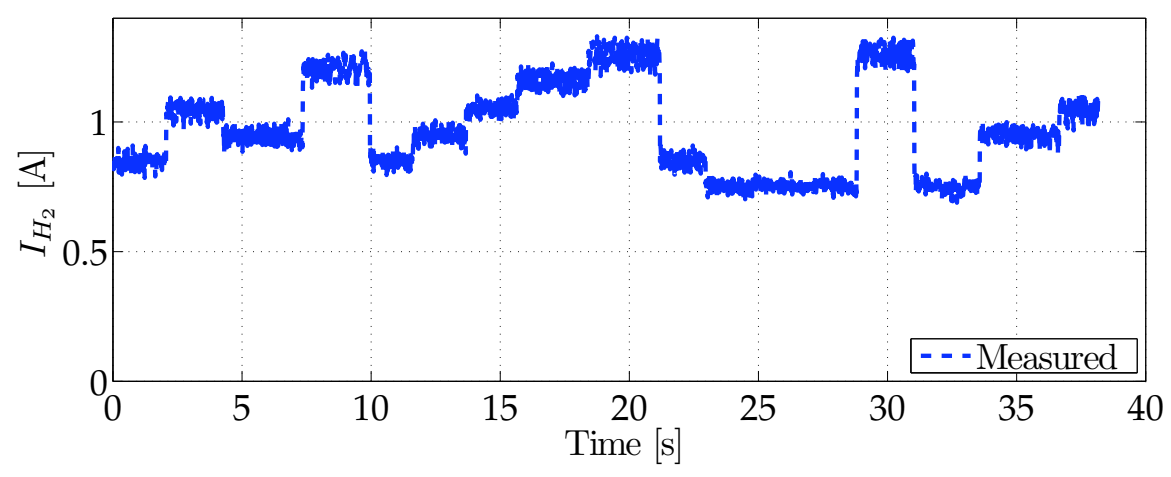

(b) Measured hydrogen pump current

Figure 5.13: Experimental results used for identification of the hydrogen supply subsystem model.

A non-linear least squares algorithm was employed to determine the characteristic hydrogen supply subsystem parameters. Their respective values are represented in Table 5.4.

\subsubsection{Model Validation}

The data used for model validation is shown in Figure 5.14 along with the modelled variables.

Excellent agreement between modelled and experimental hydrogen flow rates is observed. The average prediction error is below $5 \%$. Note that 


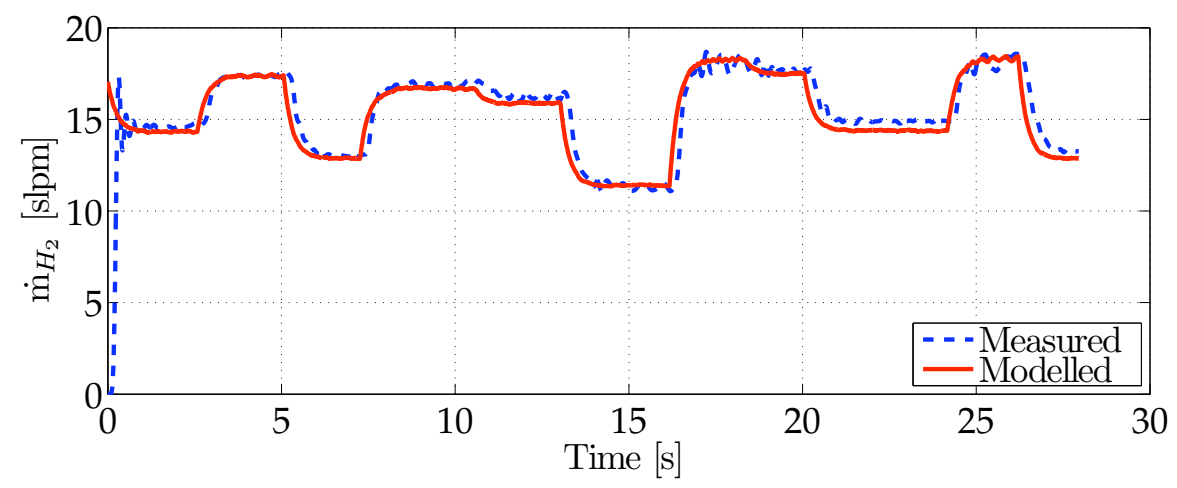

(a) Modelled and measured hydrogen mass flow through the fuel cell

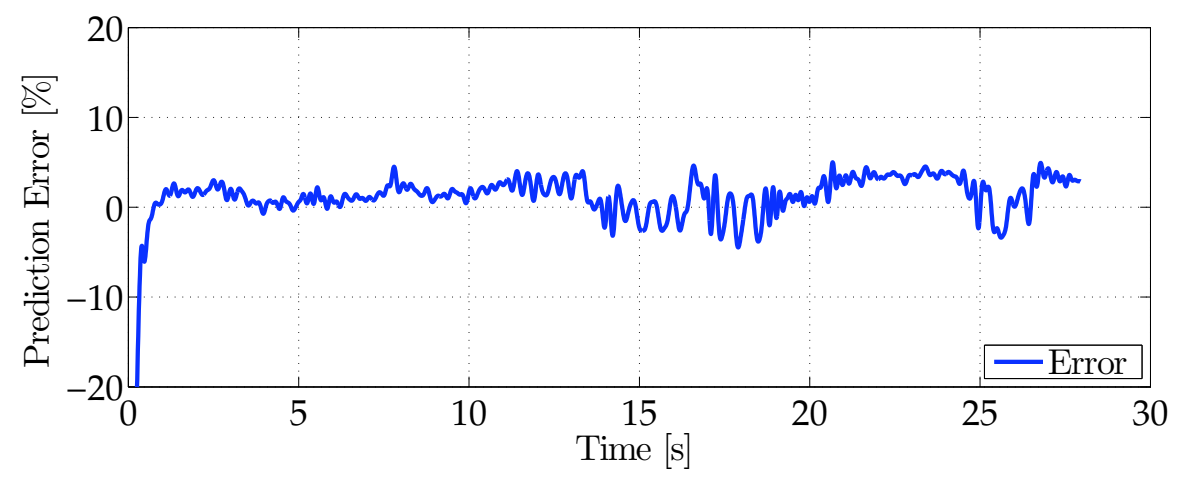

(b) Prediction error for the hydrogen mass flow

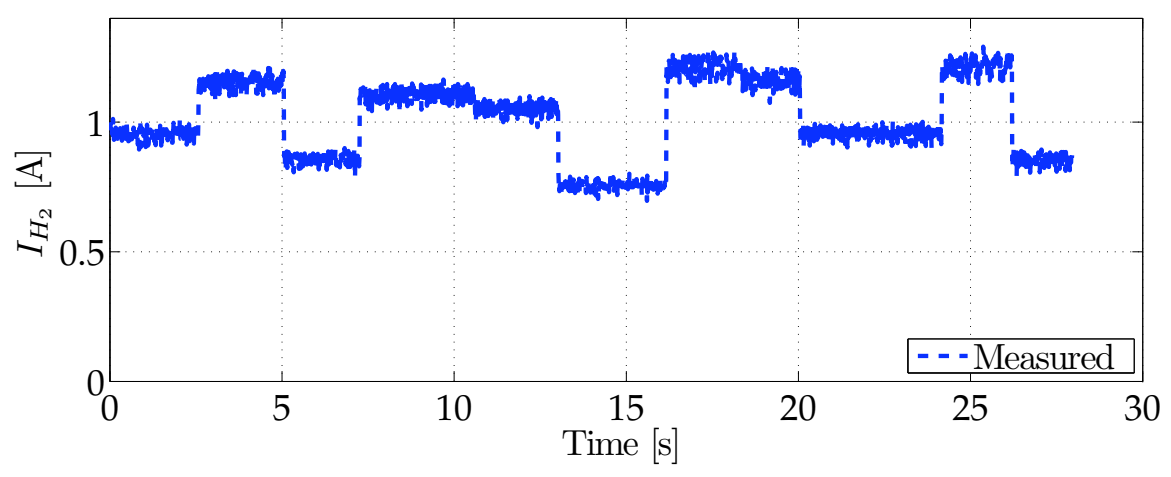

(c) Measured hydrogen pump current

Figure 5.14: Experimental results used for the verification of the hydrogen supply subsystem model. 


\begin{tabular}{lccc}
\hline Description & Parameter & Value & Units \\
\hline Motor torque constant & $k_{t, H P}$ & $6 \cdot 10^{-2}$ & $\frac{\mathrm{Nm}}{A}$ \\
Equivalent inertia & $J_{H P}$ & $1.26 \cdot 10^{-4}$ & $\mathrm{~kg} \cdot \mathrm{m}^{2}$ \\
Friction coefficient & $k_{f, H P}$ & $5.25 \cdot 10^{-4}$ & $\mathrm{Nm} \frac{\mathrm{s}}{\mathrm{rad}}$ \\
Speed-flow rate coefficient & $k_{m, H P}$ & 0.132 & $\frac{\mathrm{slpm} \cdot \mathrm{s}}{\mathrm{rad}}$ \\
\hline
\end{tabular}

Table 5.4: Experimentally identified hydrogen supply subsystem parameters.

because of a limitation in open loop performance of the current controller, the dynamic model could only be validated for hydrogen flow rates above 10 slpm.

The observed mechanical time constant of around $0.3 s$ is confirmed by the model corresponding to the data shown in Figure 5.14. The state space matrices are given by:

$$
\begin{gathered}
{\left[\frac{d \omega_{H P}}{d t}\right]=[-1.99]\left[\omega_{H P}\right]+[227]\left[i_{H P}\right]} \\
{\left[\dot{m}_{H_{2}}\right]=[0.132]\left[\omega_{H P}\right]}
\end{gathered}
$$

\subsubsection{Pressure Dynamics in the Hydrogen Supply Manifold}

The hydrogen pressure dynamics in the supply manifold have been outlined and simulated in chapter 3.4.3. This was experimentally verified on the running fuel cell through a step change in fuel cell current as shown in Figure 5.15.

In order not to mask the pressure readouts in the pressure fluctuations caused by the hydrogen recirculation pump, the fuel cell was run in deadend mode during this experiment - i.e. no hydrogen recirculation took place.

Note that the slope of the pressure reduction after the current step change corresponds to the ratio of the fuel cell current to the total hydrogen supply manifold volume. A similar steady state pressure error to the one found in chapter 3.4.3 is observed. 


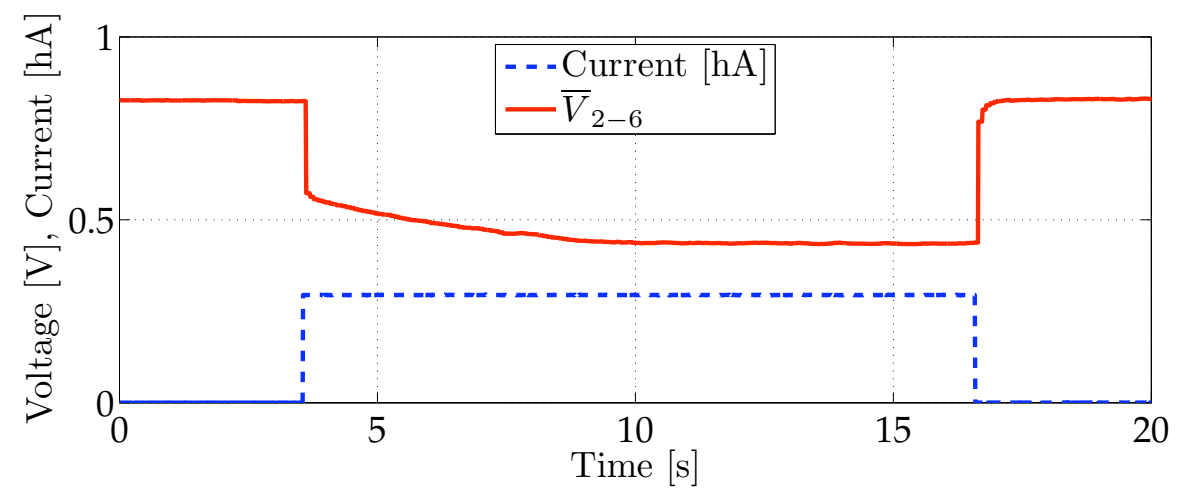

(a) Fuel cell current and voltage

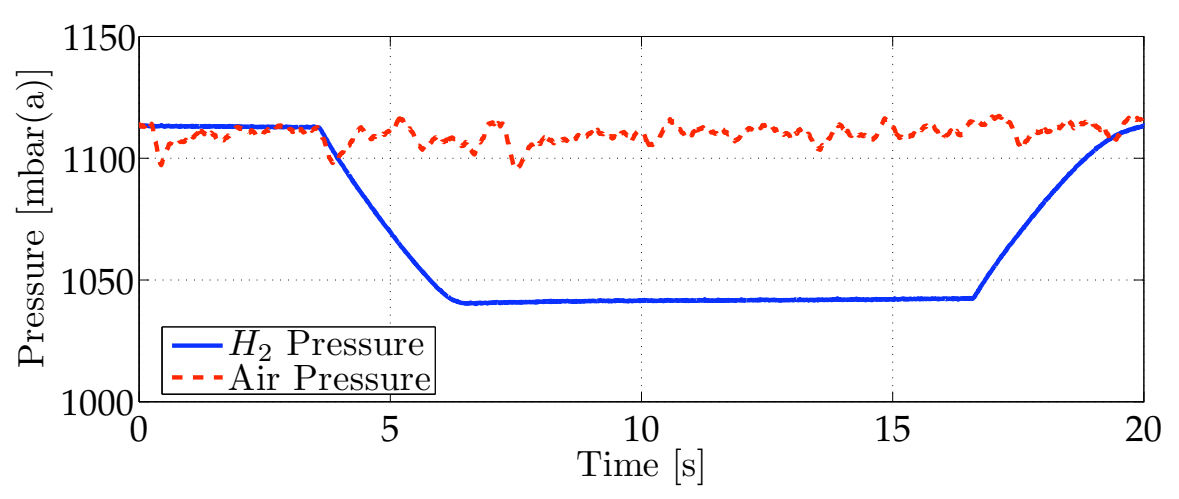

(b) Average anode and cathode pressures

Figure 5.15: Experimental demonstration of the dynamics of the pressure regulation system in the hydrogen supply manifold. 


\subsubsection{Summary}

The hydrogen supply subsystem parameters have been identified based on dynamic experimental measurements.

The model has been found to accurately predict the hydrogen mass flow evolution as a function of the applied pump current. The prediction error in the upper half of the flow rate range has been shown to be inferior to $5 \%$. Mechanical time constants around $0.3 s$ were correctly predicted by the model.

Additional experimental data confirmed the dynamics of the hydrogen pressure within the supply manifold when subjected to varying fuel cell currents. The mechanical pressure regulation valve cannot maintain constant hydrogen pressure under such operating conditions.

\subsection{Thermal Management Subsystem}

The model for the thermal management subsystem established in chapter 3.5 describes the evolution of the cooling liquid reservoir and fuel cell stack temperature as a function of the fuel cell losses, the heating power supplied to the heating cartridge, the voltage applied to the CL pump, the chill water and ambient temperatures and the chill water valve duty cycle ratio.

The equations describing the system are strongly non-linear and have been linearised around a given operating point to allow representation of the system in matrix form.

This chapter presents the experimental results used for parameter identification followed by a section on model validation based on additional experimental data.

\subsubsection{Parameter Identification}

This section outlines the techniques and data used for parameter identification of the thermal management subsystem. Where possible, dedicated measurements were used to identify a given parameter as opposed to using data to batch-identify a set of parameters.

\section{Cooling Liquid Pump Parameter Identification}

The turbine flow meter used to measure the cooling liquid flow rate cannot reliably measure flow rate dynamics. Therefore, steady state flow rate, 
current and voltage measurements combined with manual pump speed data collection was used to identify most of the cooling liquid pump parameters.

The resulting measurements are represented graphically in Figure 5.16. The torque constant $k_{t, C P}$ of the cooling liquid pump's electric motor is the fitting parameter for the left hand side graph, showing excellent agreement between modelled and experimental data. The corresponding value for $k_{t, C P}$ is indicated in Table 5.5.
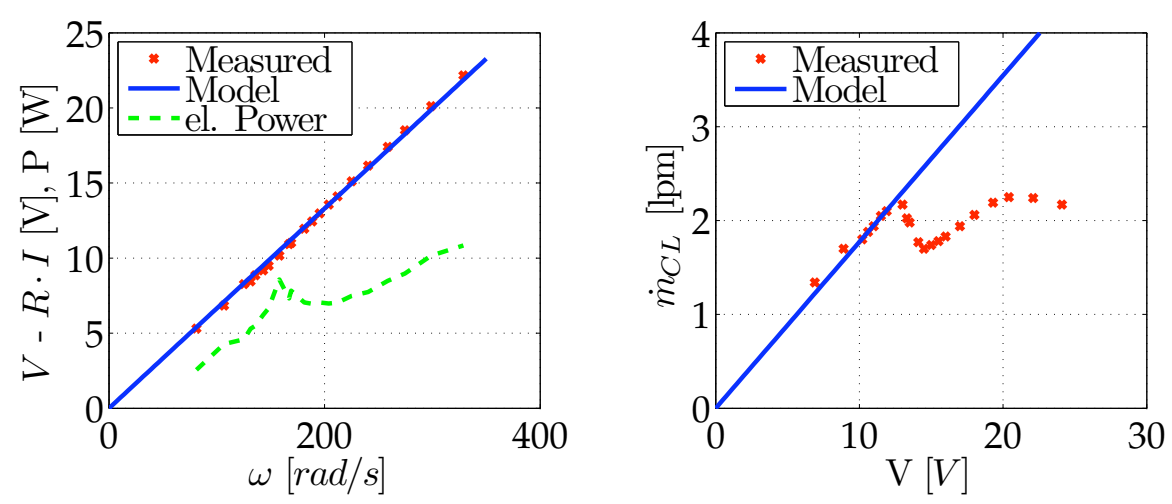

Figure 5.16: Identification of the cooling liquid pump motor's torque constant $k_{t, C P}$ and speed-mass flow coefficient $k_{m, C P}$.

The right hand side of Figure 5.16 shows the CL flow rate against the applied pump voltage. Note that there is a pronounced non-linearity in the flow regime. However, since the linear part from $0-160 \mathrm{rad} / \mathrm{s}$ (corresponding to an applied pump voltage between 0 and $12 \mathrm{~V}$ ) already reaches the maximum flow rate, there is no point in running the pump beyond that regime where the power consumed by the pump will increase further.

Additional experimental data was used in conjunction with the data represented in Figure 5.16 to determine the speed-flow rate coefficient $k_{m, C P}$, the voltage-flow rate coefficient $k_{C P}$ and the friction coefficient $k_{f, C P}$. Their respective values are indicated in Table 5.5.

\section{Thermal Fuel Cell Parameter Identification}

The cooling channel surface can be directly calculated from the fuel cell geometry. 14 cooling channels - each $281 \mathrm{~mm}$ long with $2.6 \mathrm{~mm}$ crosssectional circumference - in every of the 8 bipolar plates result in an overall 


\begin{tabular}{lccc}
\hline Description & Parameter & Value & Units \\
\hline Motor resistance & $R_{C P}$ & 0.6 & $\Omega$ \\
Motor torque constant & $k_{t, C P}$ & 0.067 & $\frac{N m}{A}$ \\
Friction coefficient & $k_{f, C P}$ & $2.2 \cdot 10^{-4}$ & $N m \frac{s}{r a d}$ \\
Speed-flow rate coefficient & $k_{m, C P}$ & 0.014 & $\frac{l p m \cdot s}{r_{p m}^{a d}}$ \\
Voltage-flow rate coefficient & $k_{C P}$ & 0.206 & $\frac{l_{p}}{V}$ \\
\hline
\end{tabular}

Table 5.5: Experimentally identified cooling liquid pump parameters.

cooling surface of $0.082 \mathrm{~m}^{2}$. The internal convection coefficient is given by the channel geometry and flow conditions. It can be shown that for the cooling liquid flow rates considered, laminar flow conditions exist in the cooling channels. This translates into constant Nusselt numbers [57] for the given channel geometry and thus constant ${ }^{7}$ convection coefficients around $h_{F C}=4000 \frac{W}{K \cdot m^{2}}$.

The equivalent specific heat and the fuel cell mass is determined through the mass and specific heat of the bipolar plates for an optimised stack. The stack used on the LEI test stand was assembled with solid aluminium end plates ${ }^{8}$ since stack weight is not an issue in a laboratory environment. With only seven cells, this results in an end plate mass substantially larger than the contribution of the bipolar plates. Averaging the contributions, a total fuel cell mass of $m_{F C}=9 \mathrm{~kg}$ and overall heat capacity of $c_{p, F C}=920 \frac{\mathrm{J}}{\mathrm{kg} \cdot \mathrm{K}}$ are found.

In the fuel cell configuration with solid end plates, the bulk of the heat transfer through natural convection occurs through the end plates. Because the heat transfer into the end plates takes place through the adjacent bipolar plate, this leads to non-uniform temperature distributions across the stack. This fact is not accounted for in the model. The end plates themselves with a Biot number of around 0.001 can be considered isothermal [57].

If the system can be approximated by a lumped capacitance description, the thermal time constant with respect to convective heat transfer can be

\footnotetext{
${ }^{7}$ The convection coefficient depends also on the thermal cooling liquid conductivity. However, the variation over the considered temperature range is below $3 \%$ and has been neglected.

${ }^{8}$ They can be produced substantially cheaper than the weight optimised end plates designed at ETHZ [34].
} 
expressed $\mathrm{as}^{9}$ :

$$
\tau_{\text {conv }}=\frac{m \cdot c_{p}}{h \cdot A_{s}}
$$

If the end plate mass is assumed to be negligible with respect to the overall stack mass, a time constant (with respect to forced internal convection) of $\tau_{\text {conv }} \approx 5 \mathrm{~s}$ result. If the end plate mass is included, the time constant becomes $\tau_{\text {conv }} \approx 25 \mathrm{~s}$. Considering only external convection, a time constant of $\tau_{\text {conv }} \approx 3 h$ is found for the stack configuration installed at LEI - i.e. with the heavy aluminium end plates.

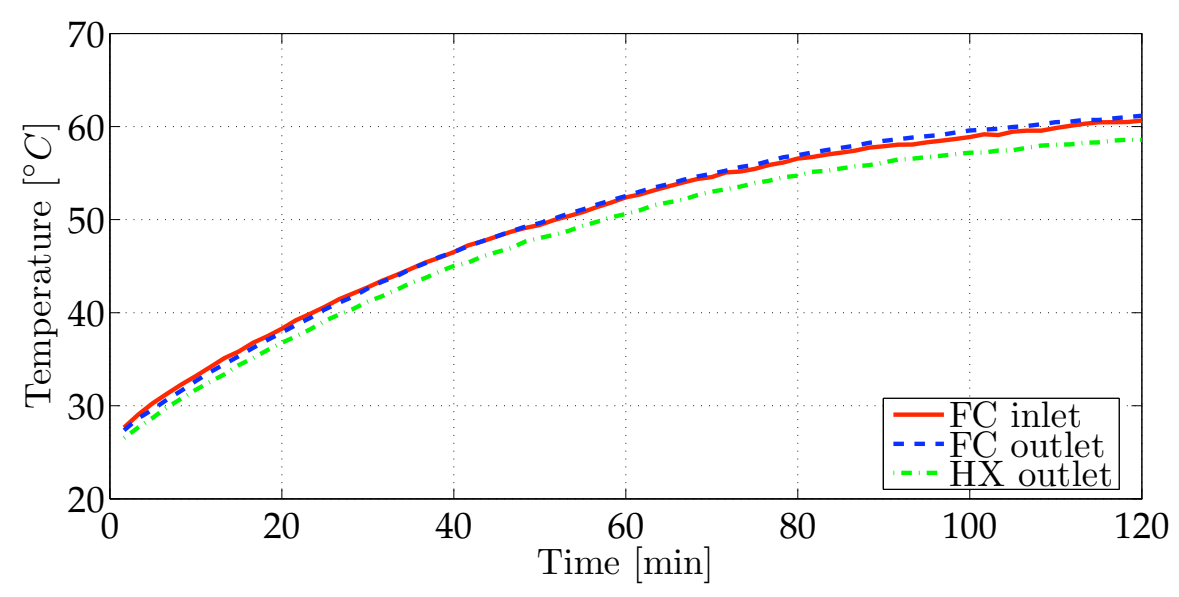

Figure 5.17: Experimental temperature evolution within the cooling circuit when subjected to a constant heating power of $200 \mathrm{~W}$. The cooling liquid flow rate is $\dot{m}_{C L}=1.1 \mathrm{lpm}$. The corresponding heat transferred from the CL is shown in Figure 5.19.

Figure 5.17 depicts the temperature evolution in the system when subjected to a constant heating power of $200 \mathrm{~W}$. The natural convection coefficients $k_{F C}$ and $k_{R V}$ can be identified through an energy balance from the steady state response.

The overall natural convection coefficient $k_{R V}$ - regrouping the contributions of the tubing, heat exchanger and reservoir - is around $5.8 \mathrm{~W} / \mathrm{K}$. This is obtained from the observed temperature drop of roughly $2.5^{\circ} \mathrm{C}$ at a CL flow rate of $\dot{m}_{C L}=1.1 \mathrm{lpm}$ corresponding to a convective heat transfer of $180 \mathrm{~W}$.

\footnotetext{
${ }^{9}$ See section 3.5.2 for more details.
} 
The temperature sensor precision is not sufficient to determine the natural convection heat transfer coefficient $k_{F C}$ for the fuel cell. However, the power difference between the electrical heating power and the one dissipated to the environment by the other components confirms the theoretically determined heat transfer coefficient (see chapter 3.5.2) of $k_{F C}=0.7 \mathrm{~W} / \mathrm{K}$.

Table 5.6 contains the characteristic thermal fuel cell parameters along with their values obtained through parameter identification or direct calculation.

\begin{tabular}{lccc}
\hline Description & Parameter & Value & Units \\
\hline Natural convection coefficient & $k_{F C}$ & 0.7 & $\frac{W}{K}$ \\
Overall fuel cell mass & $m_{F C}$ & 8.8 & $k g$ \\
Equivalent specific heat & $c_{p, F C}$ & 920 & $\frac{J}{k \cdot \cdot K}$ \\
Internal convection coefficient & $h_{F C}$ & 3990 & $\frac{W}{m^{2} \cdot K}$ \\
Cooling channel surface & $A_{s, F C}$ & 0.082 & $\mathrm{~m}^{2}$ \\
\hline
\end{tabular}

Table 5.6: Experimentally identified bipolar plate parameters.

\section{Cooling Liquid Reservoir Parameter Identification}

Direct calculation allows to determine the cooling liquid reservoir mass (steel part) $m_{R V}=1.41 \mathrm{~kg}$ along with the total cooling liquid mass $m_{C L}=0.5 \mathrm{~kg}$. However, since the CL reservoir parameters regroup the contributions from the tubing and heat exchanger ${ }^{10}$, experimental identification is required to obtain the overall parameters.

The natural convection coefficient for the CL reservoir only is obtained by heating the system to a certain temperature and then letting it cool down with no cooling liquid flow. The results are shown in Figure 5.18 and yield a value of $k_{R V \text {, only }}=0.52 \mathrm{~W} / \mathrm{K}$.

However, the model assumes that the natural convection coefficient $k_{R V}$ regroups the natural convection contributions of the tubing, the heat exchanger and the CL reservoir. It was shown from measurements represented in Figure 5.17 that the overall convective heat transfer coefficient is roughly ten times larger than the one identified for the CL reservoir alone. Further measurements (see Figure 5.20) were used with a non-linear

\footnotetext{
${ }^{10}$ This applies only to natural convection and thermal inertia terms.
} 


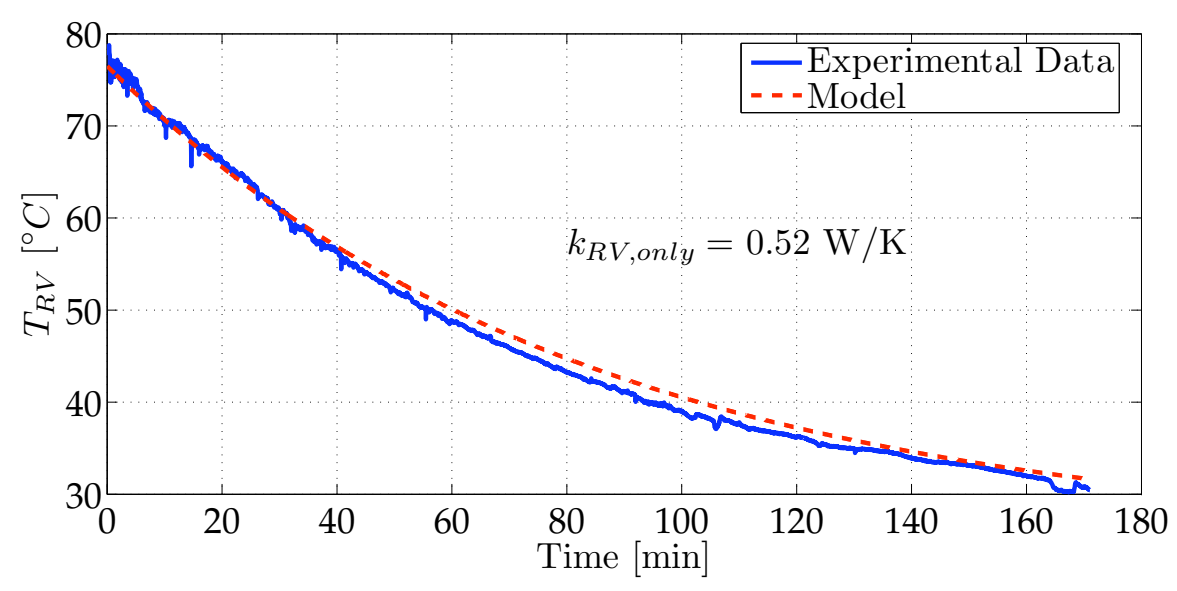

Figure 5.18: Temperature evolution of the CL reservoir when cooling from an initial temperature with no cooling liquid flow.

least squares algorithm to determine the equivalent overall convective heat transfer coefficient $k_{R V}$.

The same data as used in Figure 5.17 can be rearranged to show the heat withdrawn from the cooling liquid from reservoir exit to re-entry as a function of the temperature difference between ambient and CL reservoir temperature.

Note the initial heat transfer of around $50 \mathrm{~W}$. It corresponds to the heat extracted from the CL to heat the components mass. On approaching steady state conditions, heat is transferred through natural convection to the environment. The corresponding characteristic CL reservoir parameters are shown in Table 5.7.

\begin{tabular}{lccc}
\hline Description & Parameter & Value & Units \\
\hline Cooling liquid mass & $m_{C L}$ & 0.475 & $\mathrm{~kg}$ \\
Cooling liquid reservoir mass & $m_{R V}$ & 1.41 & $\mathrm{~kg}$ \\
Cooling liquid specific heat & $c_{p, C L}$ & 4180 & $\frac{\mathrm{J}}{\mathrm{kg} \cdot \mathrm{K}}$ \\
Cooling liquid reservoir specific heat & $c_{p, R V}$ & 450 & $\frac{\mathrm{J}}{\mathrm{kgKK}}$ \\
Natural convection coefficient & $k_{R V}$ & 4.8 & $\frac{\mathrm{W}}{K}$ \\
\hline
\end{tabular}

Table 5.7: Experimentally identified cooling liquid reservoir parameters. 


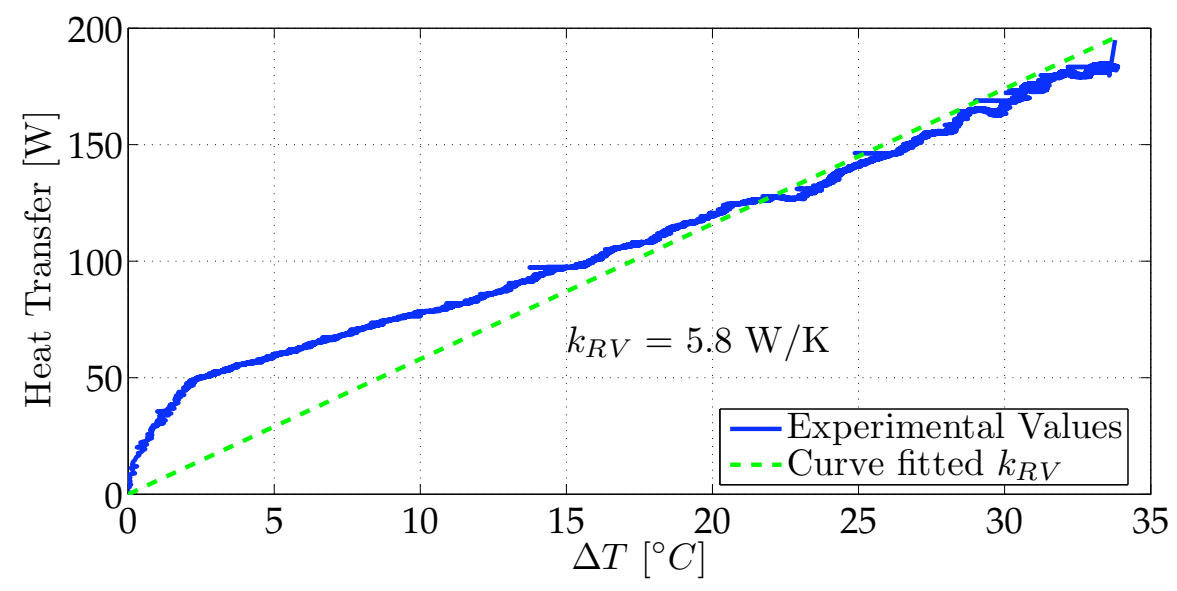

Figure 5.19: Heat transfer from the cooling liquid when subjected to a constant heating power of $200 \mathrm{~W}$. The cooling liquid flow rate is $\dot{m}_{C L}=1.1 \mathrm{lpm}$. The corresponding temperature evolution is shown in Figure 5.17.

\section{Heat Exchanger Parameter Identification}

The liquid-liquid heat exchanger rejects heat from the cooling liquid to a secondary chill water circuit which is fed through a controllable electromagnetic valve $(\mathrm{CWV})$. The valve can be switched on and off with a variable duty cycle at a user adjustable frequency.

Experimental data shown in Figure 5.20 were used to determine the equivalent natural convection coefficient $k_{R V}$ and the heat exchanger efficiency-flow rate coefficient $k_{x}$ through a non-linear least squares algorithm.

During the identification procedure, an averaged state space system was recalculated every 50 seconds to take changing average temperatures, chill water valve duty cycles and CL flow rates. The resulting heat exchanger efficiency-flow rate coefficient $k_{x}$ is shown in Table 5.8.

\begin{tabular}{lccc}
\hline Description & Parameter & Value & Units \\
\hline Efficiency-flow rate coefficient & $k_{x}$ & 0.04 & $s / k g$ \\
\hline
\end{tabular}

Table 5.8: Experimentally identified cooling liquid reservoir parameters.

It was found that for the considered operating conditions, the model is quite insensitive to the actual heat exchanger efficiency. Both changing the 


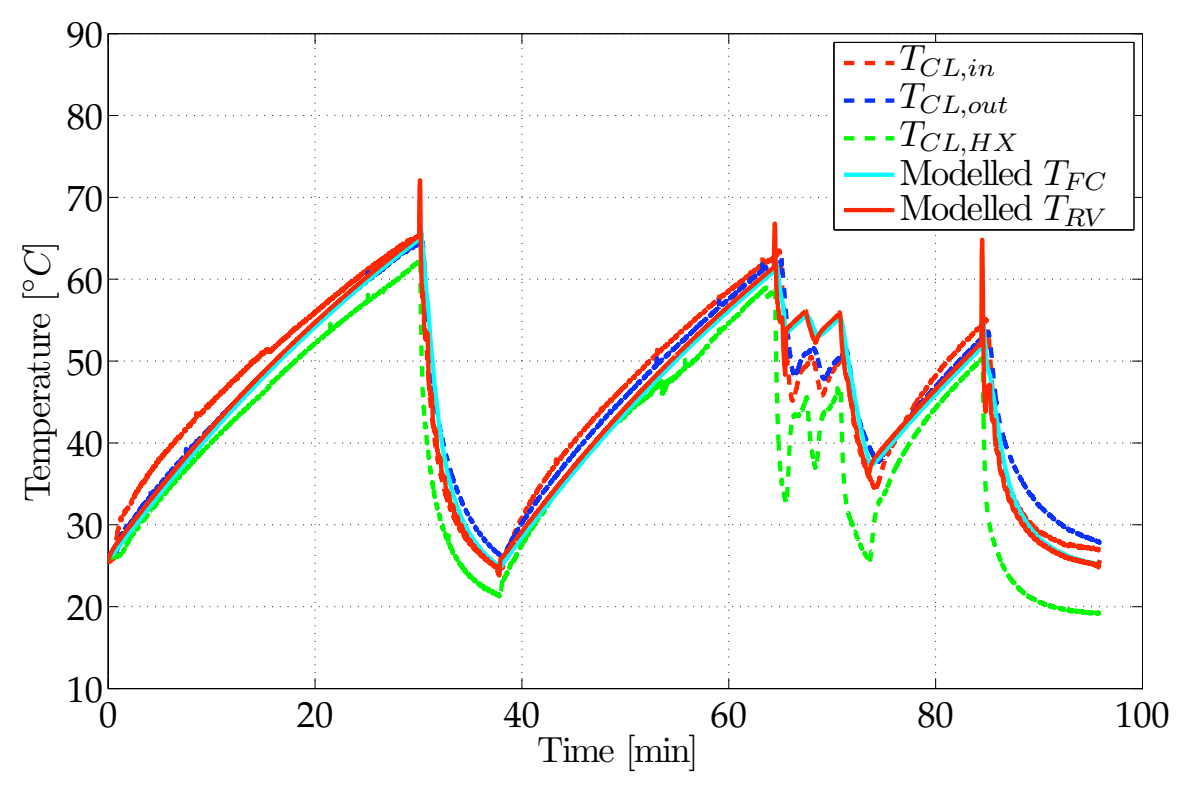

(a) Modelled and measured temperatures in the cooling circuit

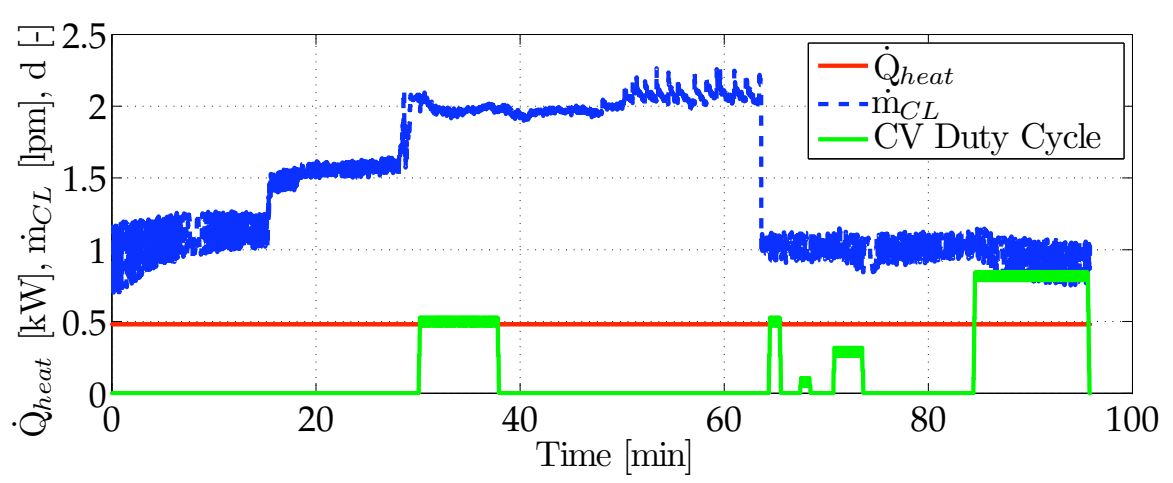

(b) Measured inputs to the cooling circuit model

Figure 5.20: Experimental results used for the identification of the heat exchanger parameters. 
base efficiency $\epsilon_{0}$ and the efficiency flow-rate coefficient $k_{x}$ only produced minor changes in the predicted temperatures.

\subsubsection{Model Validation}

The experimental data used for validation of the cooling liquid pump is shown in Figure 5.21. The time delay observed between measured and experimental flow rate in Figure 5.21(a) is due to the readout procedure from the mass flow sensor.

Discounting for that time delay, excellent model performance with prediction errors inferior to $10 \%$ is typically encountered. Prediction of the power consumption of the cooling liquid pump also shows very good agreement with experimental data.

Figure 5.22 shows the experimental data used for validation of the overall cooling circuit model presented in chapter 3.5.8. Good agreement between experimental and modelled data is observed. Typical prediction errors are $10 \%$ when expressed in degrees Celsius.

Because the overall system model is a linearised description of a nonlinear system, the state space equations need to be adjusted to the actual average operating conditions. It was found that model accuracy depends strongly on the average duty cycle ratio being close to the actual value - the dependency on average temperatures and CL flow rate is far less pronounced.

This is also represented in the state space equations for a given operating point. If the chill water valve is closed, the cross coupling between the fuel cell and RV temperature is less pronounced. Additionally, the time constants are dominated by convective heat transfer. This translates into time constants of around $\tau_{1} \approx 3^{\prime} 000 \mathrm{~s}$ and $\tau_{2} \approx 13 \mathrm{~s}$. For an average temperature of $\bar{T}_{F C}=\bar{T}_{R V}=60^{\circ} \mathrm{C}$ and a CV duty cycle ratio of $d=0$, 


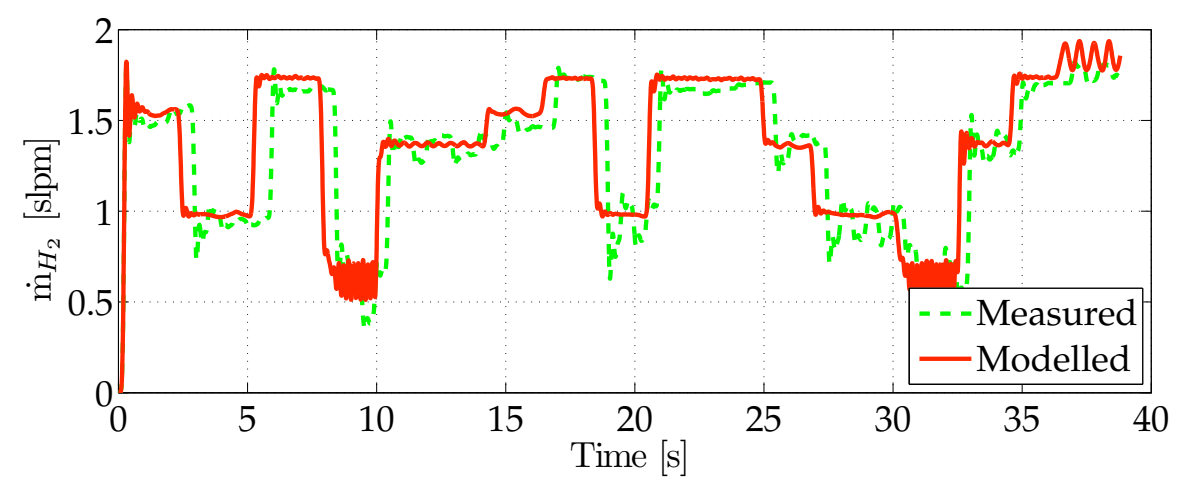

(a) Modelled and measured cooling liquid mass flow

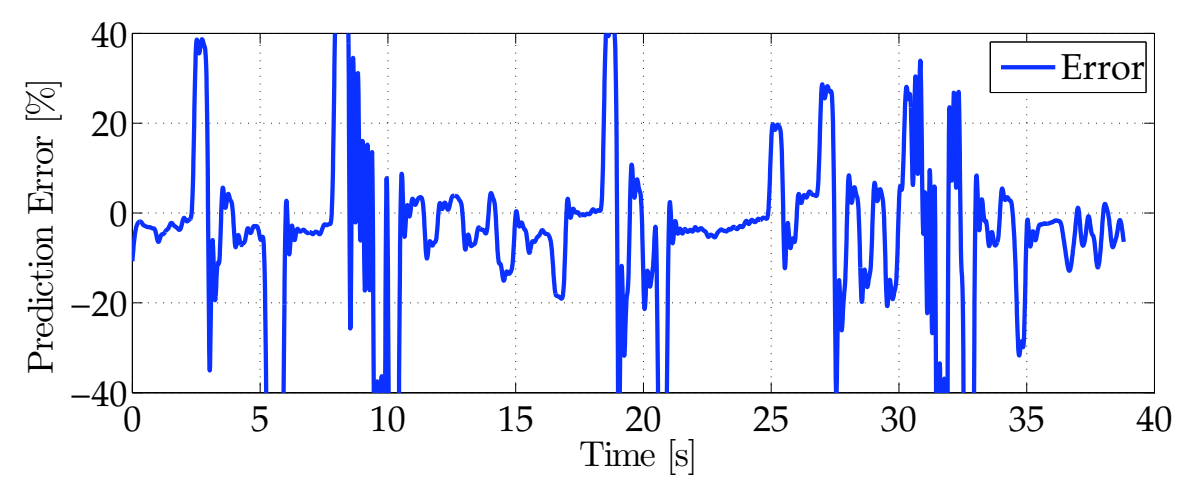

(b) Prediction error for the cooling liquid mass flow

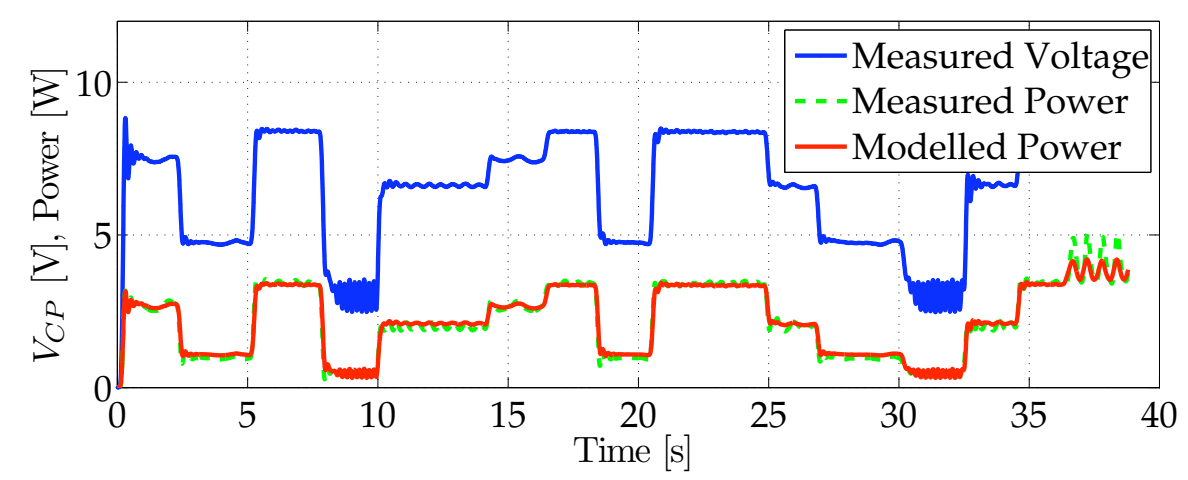

(c) Modelled and measured CL pump power consumption and measured CL pump voltage

Figure 5.21: Experimental results used for the validation of the cooling liquid pump model. 


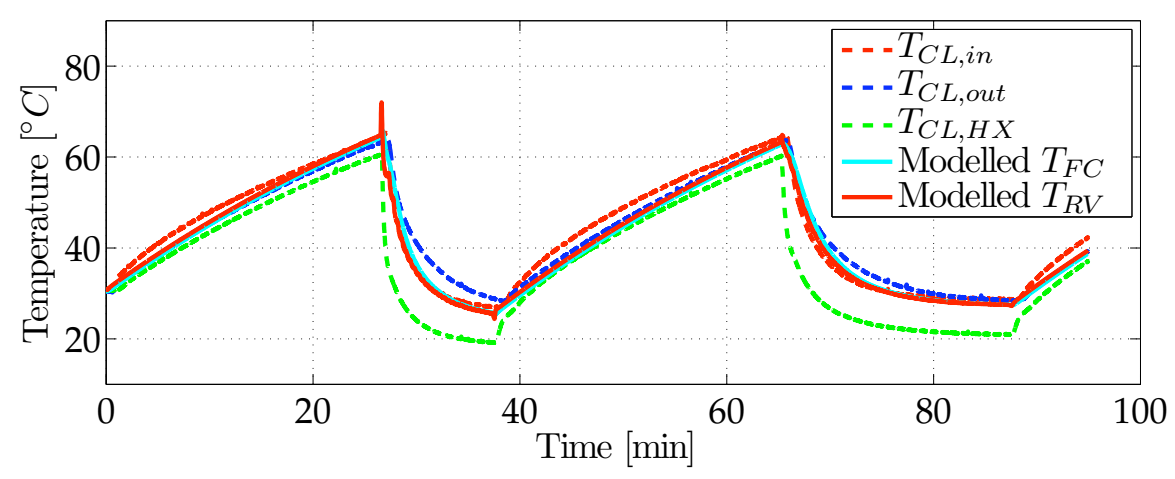

(a) Modelled and measured temperatures in the cooling circuit

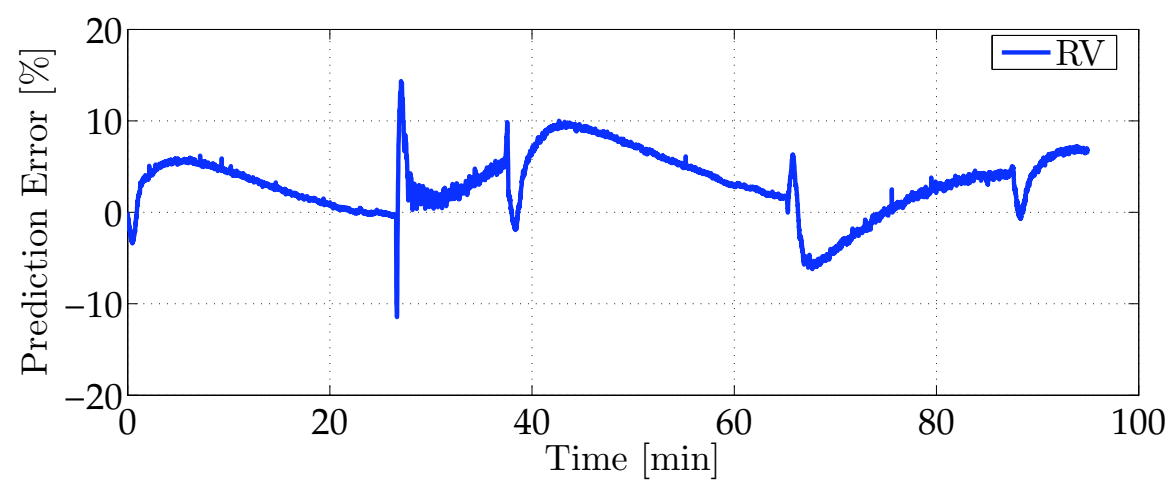

(b) Prediction error for the cooling liquid reservoir temperature

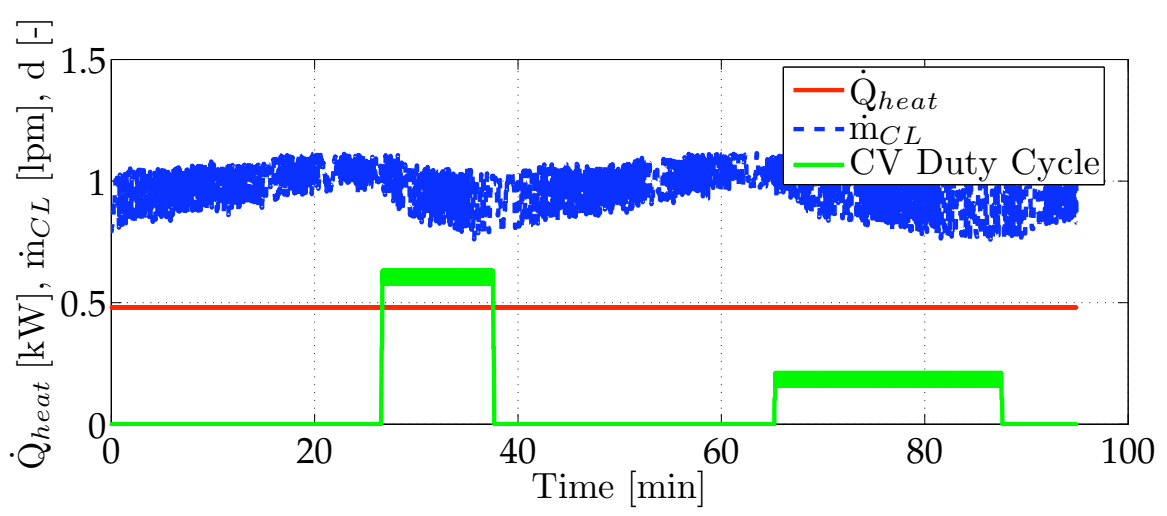

(c) Measured inputs to the cooling circuit model

Figure 5.22: Experimental results used for the validation of the heat exchanger parameters. 
the corresponding state space equations are given by:

$$
\begin{aligned}
{\left[\begin{array}{c}
\frac{d \tilde{T}_{F C}}{d t} \\
\frac{d T_{R V}}{d t}
\end{array}\right]=} & {\left[\begin{array}{cc}
-0.04 & 0.04 \\
0.04 & -0.04
\end{array}\right]\left[\begin{array}{c}
\tilde{T}_{F C} \\
\tilde{T}_{R V}
\end{array}\right] } \\
& +\left[\begin{array}{cccccc}
0.0001 & 0 & 0 & 0 & 0.00007 & 0 \\
0 & 0.0001 & 0 & 0 & 0.0006 & 0
\end{array}\right]\left[\begin{array}{c}
\tilde{\dot{Q}}_{\text {loss }} \\
\tilde{Q}_{\text {heat }} \\
\tilde{V}_{C P} \\
\tilde{T}_{C W} \\
\tilde{T}_{a m b} \\
\tilde{d}
\end{array}\right]
\end{aligned}
$$

If the chill water valve duty cycle is non-zero, forced internal convection dominates and the resulting time constants become $\tau_{1} \approx 30 \mathrm{~s}$ and $\tau_{2} \approx 4 \mathrm{~s}$. Additionally, a strong coupling between the fuel cell and RV temperature exists as seen in the increased value of $\mathbf{A}_{(2,2)}$. The state space equations corresponding to an average temperature of $\bar{T}_{F C}=\bar{T}_{R V}=50^{\circ} \mathrm{C}$ and a $\mathrm{CV}$ duty cycle ratio of $d=0.5$ are given by:

$$
\begin{aligned}
{\left[\begin{array}{c}
\frac{d \tilde{T}_{F C}}{d t} \\
\frac{d T_{R V}}{d t}
\end{array}\right]=} & {\left[\begin{array}{cc}
-0.04 & 0.04 \\
0.03 & -0.25
\end{array}\right]\left[\begin{array}{c}
\tilde{T}_{F C} \\
\tilde{T}_{R V}
\end{array}\right] } \\
& +\left[\begin{array}{ccccccc}
0.0001 & 0 & 0 & 0 & 0.00007 & 0 \\
0 & 0.0001 & -3 & 0.2 & 0.0006 & 13
\end{array}\right]\left[\begin{array}{c}
\tilde{Q}_{\text {loss }} \\
\tilde{\hat{Q}}_{\text {heat }} \\
\tilde{V}_{C P} \\
\tilde{T}_{C W} \\
\tilde{T}_{a m b} \\
\tilde{d}
\end{array}\right]
\end{aligned}
$$

Note the dominance of the heat exchanger on the temperature evolution as seen in the value of $\mathbf{B}_{(2,6)}$ which reflects a change in duty cycle ratio to the observed RV temperature.

\subsubsection{Summary}

The thermal management subsystem's parameters have been identified based on different steady state and dynamic measurements.

The cooling liquid flow rate prediction of the cooling pump was found to be in very good agreement with experimental data. Prediction errors below $10 \%$ were typically observed. 
The overall thermal management subsystem model accurately predicts the evolution of the RV and FC temperatures. The linearised state space model was found to be sensitive to accurate average operating conditions, particularly for the chill water valve duty cycle ratio.

Experimentally observed time constants of around $50 \mathrm{~min}$ when heating and of around $30 \mathrm{~s}$ when cooling with elevated duty cycle ratios were correctly reproduced by the model.

With a readjustment of the average operating conditions every 50 seconds, prediction errors of less than $10 \%$ were observed. Shortening the prediction horizon further by evaluating the average operating conditions more frequently is likely to further reduce the prediction errors.

\subsection{Conclusion}

This chapter has presented experimental results to validate the fuel cell stack and balance of plant models developed in chapters 2 and 3.

The first section focused on the fuel cell stack model. Because of a pronounced degradation of the fuel cell stack installed at $L E I$, the model was first validated on experimental data from literature. Very good agreement between experimental and modelled data was found. However, the small sample size did not allow the validation of the model for varying operating conditions.

Steady-state data obtained from the LEI test bench was used to determine water transport relevant parameters. Pseudo steady state IV curve data was used to determine the remaining performance parameters. Model validation showed reasonable agreement between modelled and experimental data while operating under healthy conditions. Due to the stack's degradation, open circuit potential conditions were not predicted correctly. Dry-out conditions where the model is not valid were experimentally identified.

The remaining sections focused on parameter identification and model validation for the balance of plant systems.

The air supply subsystem model was found to correctly describe the pressure and air flow rate dynamics in the inlet manifold. Typical hydraulic time constants of around $0.3 s$ were correctly predicted by the model. Flow rate prediction showed prediction errors below $10 \%$ for flow rates above 15 slpm.

The hydrogen subsystem model correctly predicts the hydrogen mass flow as a function of the applied pump current with prediction errors inferior to 
$5 \%$. The associated mechanical time constant of around $0.3 s$ is reproduced by the model. Additional experimental data confirmed ${ }^{11}$ the behaviour of the pressure regulation valve when subjected to sudden variations in cell current.

Finally, the thermal management subsystem describes the temperature dynamics in the fuel cell system with prediction errors below $10 \%$. Because the state space model has been obtained from linearisation of the governing equations, shortening the prediction horizon of the model increases its accuracy. The performance of the installed heat exchanger is such that the model becomes sensitive to variations in the average chill water valve duty cycle ratio.

\footnotetext{
$\overline{{ }^{11} \text { See chapter } 3.4 .3 \text { for more details. }}$
} 



\section{Conclusions and Future Work}




\subsection{Summary}

The goal of this thesis is to establish a control-oriented PEM fuel cell system model that describes the dynamic effects of a user interaction with any of the balance of plant actuators on overall system performance.

The first chapter introduces the subject of fuel cells. The motivation behind the present work, a detailed literature overview and a summary of the contributions of this work are presented.

In chapter 2, a control-oriented, analytical, cell-averaged, steady state fuel cell stack model is developed. The model predicts the cell voltage along with the average membrane water content as a function of the fuel cell's operating conditions.

In order to obtain a cell-averaged description of the occurring processes, an approach for determining the mean species' concentrations in the system is developed. The novelty of the calculations consists in taking the water transfer across the system into account. This forms the basis for a one-dimensional description of water and gas transport in the direction perpendicular to the membrane.

A novel approach for analytically determining the overall water transport within the fuel cell based on the operating conditions is introduced. This eliminates the traditionally required iterative procedures and constitutes an important step in enabling the model to be used in real-time control applications.

Chapter 3, focuses on modelling the auxiliary systems that supply the fuel cell with reactant gases at appropriate flow rates, pressures and humidities and control the temperature of the fuel cell. Their formal description forms the missing link between a user's action on the system and the resulting change in fuel cell performance.

Dynamic, state-space models are developed for each of the auxiliary systems - i.e. the air supply subsystem, the hydrogen supply subsystem and the thermal management subsystem. Because the corresponding governing equations are non-linear in the case of the thermal management subsystem, a linearised description around a given operating point is developed.

Chapter 4 gives an overview of the fuel cell stack used in the experimental setup. The fuel cell test bench developed at LEI is described in detail. The functions and performance of the different balance of plant components are explained and the functions of the data acquisition and control softand hardware are discussed. 
The developed fuel cell stack and balance of plant models are then compared to experimental data in chapter 5. A general conclusion and an outlook over future work are given in sections 6.2 and 6.3 .

\subsection{Discussion}

The analytical, control-oriented state space model for a PEM fuel cell system developed in this thesis describes the evolution of system performance as a function of the user's interaction with the actuators.

In order to provide such an overall description, the model was elaborated in two distinct parts:

- Dynamic, balance of plant models describe the effect of a user interaction with the balance of plant systems on the resulting fuel cell operating conditions.

- A steady state, cell averaged fuel cell stack model describes the effects of the operating conditions on stack performance.

\section{Balance of Plant Models}

The balance of plant models have been developed based on the configuration installed on the $L E I$ test bench. For the air supply subsystem, this involves the description of a non-pressurised cathode compartment that is fed with ambient air from a volumetric air compressor.

The hydrogen supply subsystem is composed of a pressurised - through a mechanical pressure regulator - supply manifold where hydrogen is recirculated by a volumetric pump. For the thermal management subsystem, the model is based on a liquid cooled stack configuration where the external interface is constituted by either a liquid-liquid or liquid-air heat exchanger.

$$
* * *
$$

The proposed models have shown excellent agreement with experimental data. Experimental and modelled data have clearly exposed how the dynamic limitations of the balance of plant systems are reflected to the fuel cell stack. 
The dynamics of the mechanical pressure regulators used to impose the anode compartment pressure level have not been modelled in the context of this thesis. However, its performance limitations have been outlined and experimentally confirmed. It was shown that the pressure level is maintained with a steady state error under load conditions. The dynamics of the pressure variation are given by the ratio of the fuel cell current over the anode manifold volume.

Many fuel cell systems work with a pressurised cathode compartment. This is achieved by installing a backpressure regulating valve at the fuel cell exit. While such a system configuration has not been modelled in this thesis, problems with the description of the backpressure regulators have been investigated, outlined and experimentally verified.

\section{Fuel Cell Stack Model}

The gas transport and electrochemical reaction dynamics within the fuel cell can be shown to be noticeably faster than those of the associated balance of plant systems. Therefore, the fuel cell stack model, that relates the operating conditions to the resulting cell voltage, represents steady state behaviour.

While this steady state description is fine for relatively fast gas transport phenomena - where the balance of plant system are actually the dynamically limiting factor - it is not correct for water transport where the polymer membrane acts like a storage element and introduces slow dynamic behaviour. The time constants are typically in the order of several minutes. One of the consequences is the typically observed difference between current voltage curves recorded with positive current steps and those recorded with negative current steps. As has been shown by Opekar et al. [65], the water uptake by Nafion is much faster than its release from the membrane, adding further complexity to a dynamic description of the water transfer mechanism.

The description of the not well-understood water uptake dynamics is one of the key elements to describe the dynamics of a fuel cell stack due to a change in operating conditions. Note however, that the experimentally observed variations in cell voltage due to those dynamics, are generally small $(<5 \%)$. The steady state model thus gives a reasonable appreciation of the system performance. 
In order to obtain an analytical expression of the occurring species transport processes within the fuel cell stack, the across-the-cell transport was described in terms of average species concentrations. These were obtained by assuming a uniform current density and therefore taking the arithmetic average along the gas channel between the cell inlet and outlet.

While the assumption of an average condition, upon which the transport equations are based, is most certainly not correct in reality, a cell designer will try to achieve as uniform a current density distribution as possible to obtain the best achievable performance. In this light, for a well-built fuel cell stack, the assumption of a uniform current density is justified.

One of the key contributions of this thesis was the development of an analytical expression describing water transport within the fuel cell stack. The main assumption in its development concerns the consideration of a linear membrane water sorption characteristic while literature data presents important variations in slope. However, the linear description correctly depicts the qualitative evolution of the membrane water content over the whole operating range. This is an essential property if the representation is to be used for model-predictive control applications.

The model has been fitted to experimental literature data. A very good fit was found, but the sample size was too small to confirm a model validity over wide operating ranges.

Due to a severe degradation of the fuel cell stack installed at LEI, only few experimental data could be collected to validate the fuel cell stack model. The range of covered operating conditions was limited and not representative of the conditions a healthy stack would operate under.

The degradation of the stack was such that the modelling assumptions for the three phase boundary do not seem realistic. Therefore, the model was unable to correctly predict the cell voltage at very low current densities.

For the remaining current densities, the model has shown good agreement with a set of limited experimental data. Under severe dry-out conditions due to high fuel cell temperatures combined with high stoichiometric air flow rates and reduced dew point temperatures - the model was found to be unable to predict experimental data. This is believed to be due to excessive dry-out of not only the membrane but also the catalyst layer, resulting in reduction in three phase boundary reaction sites. 
While encouraging model performance was identified, further experimental model validation on a healthy fuel cell stack is necessary to ascertain the model's capability to predict changes in cell voltage due to a change in operating conditions and to identify the boundaries of model validity.

The model can be adapted to different PEM fuel cell systems by applying the outlined modelling methodology for any balance of plant systems that differ from the ones detailed herein. The fuel cell stack model is adjusted to the new cell configuration by fitting the model parameters.

\subsection{Future Work}

An analytical, control-oriented state space PEM fuel cell model has been elaborated in this thesis. The model provides dynamic descriptions of the auxiliary systems to determine the effects of a user's interaction with the balance of plant systems on the fuel cell stack's operating conditions.

An analytic, computationally non-intensive model was developed to resolve the dependency of the fuel cell stack performance on the operating conditions.

This work forms the basis for developing advanced model-based control strategies for PEM fuel cell systems. It is considered as a starting point for work in this area. A need for future contributions has been identified in four areas:

- Addition of dynamic descriptions of auxiliary systems not considered here. This concerns primarily the electro-mechanical pressure regulators used in fuel cell systems with pressurised cathode compartments.

While current literature models describe the pressure evolution as a function of the opening area in the pressure reducer, no description for linking the opening area to a user interaction exists. Problems with such a description have been outlined in this thesis.

Furthermore, the description of any humidification system's performance will constitute an important part in linking all fuel cell operating conditions to a balance of plant actuator.

- Extensive fuel cell stack model validation. The fuel cell stack model presented in this thesis relies on a certain number of simplifying assumptions. While the model has shown good agreement with 
limited experimental data, further validation with a healthy fuel cell stack over wide ranges of operating conditions is required.

This would best be combined with model comparison with more detailed, numerical modelling approaches.

- Extension of the stack model to cover dynamics. The dynamic evolution of the water content of both the membrane and catalyst layer are clearly observed on experimental cell voltage data. Therefore, it is important that these interactions can be described by a model. The complexity of the underlying mechanisms presents important challenges to the establishing of a simple model.

- Use of the developed model for the control of fuel cell systems. The developed fuel cell system models can be used for either model-based control of a given PEM fuel cell system or for implementing real time maximum efficiency point tracking algorithms. 



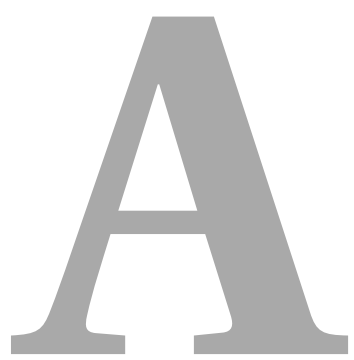

Derivation of Fuel Cell Performance Equations 


\section{A.1. Derivation of the Nernst equation}

Thermodynamics state that the maximum obtainable energy from a chemical reaction corresponds to the Gibbs free enthalpy of reaction $\Delta \mathcal{G}$. Conservation of energy directly relates the molar Gibbs free enthalpy of reaction to the maximum obtainable fuel cell voltage $E_{0}$ :

$$
\Delta \mathcal{G}=-n F E_{0}
$$

Since $\Delta \mathcal{G}$ is dependent on reactant pressure and temperature, the obtainable voltage for a given set of reaction parameters is given as a standard equilibrium voltage $E_{00}$ corrected by the deviation from those standard conditions where the deviation from standard conditions can be written as:

$$
d E_{0}=\left(\frac{\partial E_{0}}{\partial T}\right)_{p} d T+\left(\frac{\partial E_{0}}{\partial p}\right)_{T} d p
$$

\section{Temperature Dependence of the Equilibrium Voltage}

The temperature dependence of the equilibrium voltage can be derived from (2.2) and (A.1) and yields:

$$
\left(\frac{\partial E_{0}}{\partial T}\right)_{p}=-\frac{1}{n F}\left(\frac{\partial \Delta \mathcal{G}}{\partial T}\right)_{p}=\frac{\Delta \mathcal{S}}{n F}
$$

\section{Pressure Dependence of the Equilibrium Voltage}

Employing basic thermodynamic properties, (A.1) can be rewritten as:

$$
\left(\frac{\partial E_{0}}{\partial p}\right)_{T}=-\frac{1}{n F}\left(\frac{\partial \Delta \mathcal{G}}{\partial p}\right)_{T}=-\frac{\Delta V}{n F}
$$

Assuming ideal gas behaviour, the change in volume for a reaction can be written as:

$$
\Delta V=\sum_{i} \gamma_{i} \frac{\mathcal{R} T}{p_{i}}
$$

$\gamma_{i}$ is the stoichiometric factor for reactant i (i.e. $\gamma_{H_{2}} H_{2}+\gamma_{O_{2}} O_{2} \rightarrow$ $\left.\gamma_{\mathrm{H}_{2} \mathrm{O}} \mathrm{H}_{2} \mathrm{O}\right)$. It is important to note, that in this context, $\gamma_{i}$ is defined 
positively for educts and negatively for products in order to account for the way they influence the volume change during the reaction.

Including (A.5) in (A.4) yields:

$$
\left(\frac{\partial E_{0}}{\partial p}\right)_{T}=-\frac{\mathcal{R} T}{n F} \sum_{i} \frac{\gamma_{i}}{p_{i}}
$$

\section{Nernst Equation}

(A.3) and (A.6) can now be integrated from the standard temperature and pressure to the operating temperature and pressure respectively to yield:

$$
\begin{aligned}
& E_{0}=E_{00}+\frac{\Delta \mathcal{S}_{0}}{n F}\left(T-T_{0}\right)+\frac{\mathcal{R} T}{n F} \ln \left(\frac{\prod_{i}\left(\frac{p_{i}}{p_{0}}\right)^{\gamma_{i}}}{\prod_{j}\left(\frac{p_{j}}{p_{0}}\right)^{\gamma_{j}}}\right) \\
& \text { where: } E_{00}=\text { equilibrium potential at stand- } \\
& \text { ard conditions (i.e. } p_{i}=p_{0}= \\
& \left.101^{\prime} 325 \mathrm{~Pa} \text { and } T=298 \mathrm{~K}\right) \\
& p_{i}=\text { partial pressure of educt } \mathrm{i} \\
& p_{j}=\text { partial pressure of product } \mathrm{j}^{1} \quad[\mathrm{~Pa}]
\end{aligned}
$$

(2.4) is known as the Nernst equation and gives the equilibrium voltage $E_{0}$ for a given reaction as a function of temperature and reactant pressures if no current is drawn from the fuel cell. As soon as a net current is produced by the fuel cell, equilibrium conditions no longer apply and voltage losses occur in the cell.

\section{A.2. Derivation of the Butler-Volmer Equation}

An activation overpotential is associated with activation energy required to initiate a charge transfer. The losses (overpotential) will be larger the higher the rate of charge transfer (i.e. the higher the current drawn from

\footnotetext{
${ }^{1}$ For clarity and in order to free the reader from having to remember the sign change of $\gamma_{i}$ for educts and products, products are directly listed in the denominator of the logarithm.
} 
the fuel cell). This concept can be illustrated by writing a general redox reaction:

$$
\operatorname{Red} \underset{k_{b}}{\stackrel{k_{f}}{\rightleftarrows}} O x+n e^{-}
$$

For the case of the oxygen reduction reaction at the cathode, this corresponds to (A.9) and at the anode, the hydrogen oxidation reaction can be expressed as in (A.10).

$$
\begin{gathered}
O^{2-} \underset{k_{b}}{\stackrel{k_{f}}{\rightleftarrows}} \frac{1}{2} O_{2}+2 e^{-} \\
H_{2} \underset{k_{b}}{\stackrel{k_{f}}{\rightleftarrows}} 2 H^{+}+2 e^{-}
\end{gathered}
$$

$k_{f}$ and $k_{b}[\mathrm{~m} / \mathrm{s}]$ are the forward and backward reaction rates respectively. From activation energy considerations, it can be shown that

$$
k_{f}, k_{b} \propto \exp \left(\frac{-\Delta \mathcal{G}_{a c t, f, b}}{\mathcal{R} T}\right)
$$

Because the reaction occurs in an electric field, the activation energy required for a charge transfer (i.e. a charged species moving in an electric field), will be higher or lower than the chemical activation energy depending on which direction the charge transfer takes place in:

$$
\begin{gathered}
\Delta \mathcal{G}_{a c t, f}=\Delta \mathcal{G}_{\text {chem }}-(1-\beta) n F \Delta \Phi \\
\Delta \mathcal{G}_{a c t, b}=\Delta \mathcal{G}_{\text {chem }}+\beta n F \Delta \Phi
\end{gathered}
$$

Where $\Delta \Phi$ denotes the potential difference between the two states of the reaction. When no net charge transfer is occurring (i.e. at equilibrium where the forward and backward reactions balance), that potential difference is denoted $\Delta \Phi_{0}$. Defining an overpotential $\eta$ as the difference between the actual potential and the potential at equilibrium yields:

$$
\eta=\Delta \Phi-\Delta \Phi_{0}
$$

Multiplying the reaction rates (see (A.11)) by the reactant concentrations yields the molar charge flux across the interface which can be transformed 
into a current density by applying Faraday's law (2.24). This then yields the forward and backward current densities where $\Delta \mathcal{G}_{a c t, f, b}$ can be expressed by substituting (A.14) into (A.12) and (A.13):

$$
\begin{aligned}
& I_{f}^{\prime \prime}=n F c_{\text {Red }} \text { const }_{f} \exp \left(-\frac{\Delta \mathcal{G}_{\text {chem }}}{\mathcal{R} T}\right) \exp \left(+\frac{(1-\beta) n F\left(\Delta \Phi_{0}+\eta\right)}{\mathcal{R} T}\right) \\
& I_{b}^{\prime \prime}=n F c_{O x} \text { const }_{b} \exp \left(-\frac{\Delta \mathcal{G}_{\text {chem }}}{\mathcal{R} T}\right) \exp \left(-\frac{\beta n F\left(\Delta \Phi_{0}+\eta\right)}{\mathcal{R} T}\right)
\end{aligned}
$$

For equilibrium conditions (i.e. $\eta=0$ ), no net current $I^{\prime \prime}=I_{f}^{\prime \prime}-I_{b}^{\prime \prime}$ is flowing - the forward and backwards current densities have to be equal. Equalling (A.15) and (A.16) in those conditions defines an exchange current density $I_{0}^{\prime \prime}$, characteristic of the given cell:

$$
I_{0}^{\prime \prime} \equiv I_{0, f}^{\prime \prime}=I_{0, b}^{\prime \prime}
$$

While a net current is drawn from the fuel cell, the forward and backward contributions no longer cancel out. In such conditions, the net current density $I^{\prime \prime}$ is given by the difference between the forward and the backward current density ( $I_{f}^{\prime \prime}$ and $I_{b}^{\prime \prime}$ respectively). Subtracting (A.15) from (A.16) therefore yields the net current density $I^{\prime \prime}$. The resulting equation (2.7) is generally termed the Butler-Volmer (BV) equation for a redox overpotential, relating net current density in a redox reaction to the corresponding overpotential at a given electrode.

$$
I^{\prime \prime}=I_{f}^{\prime \prime}-I_{b}^{\prime \prime}=I_{0}^{\prime \prime}\left[\exp \left(-\frac{\beta n F \eta}{\mathcal{R} T}\right)-\exp \left(+\frac{(1-\beta) n F \eta}{\mathcal{R} T}\right)\right]
$$

If the overpotential of the considered electrode is positive (i.e. the actual potential is higher than the reversible potential), the forward reaction (oxidation) becomes large. The net current density is negative (electrons leave the electrode (like on the fuel cell anode). At the fuel cell cathode (oxygen reduction), the left hand (backward, reduction) term dominates.

For large overpotential values (i.e. $\left|\eta_{a c t}\right| \gg \frac{\mathcal{R} T}{\beta n F}$ or $\left|\eta_{a c t}\right| \gg \frac{\mathcal{R} T}{(1-\beta) n F}$ ), one of the exponential terms in 2.7 tends to zero. Neglecting the term approaching zero yields the Tafel equation for the cathode and anode respectively. 


\section{Anode Activation Overpotential}

For the anode reaction (1.3), the equilibrium potential $\Phi_{0}$ has been standardised at $0 \mathrm{~V}$. The effective electrode potential is above that value indicating that the anode overpotential $\eta_{a}$ is positive. The net current density is negative (i.e. electrons leaving the electrode).

\section{Cathode Activation Overpotential}

For the cathode reaction (1.4), the equilibrium potential is given by the Nernst equation (2.4) and is roughly equal to $1.23 \mathrm{~V}$ at $25^{\circ} \mathrm{C}$. The effective electrode potential is below that value indicating that the cathode overpotential $\eta_{c}$ is negative. Since oxygen is reduced to $O^{2-}$ at the cathode, the backward current density dominates and the net current density is positive (i.e. electrons entering the electrode).

\section{A.3. Limiting Current Density}

The rate of gas diffusion can be related to the current density through Faraday's law (2.24). The resulting equation can then be inserted into Fick's law (B.9) to yield:

$$
I^{\prime \prime}=n F D_{e q}\left(\frac{\partial c}{\partial z}\right)_{z=0} \approx n F D_{e q} \frac{c_{G C}-c_{S}}{\delta_{G D L}}
$$

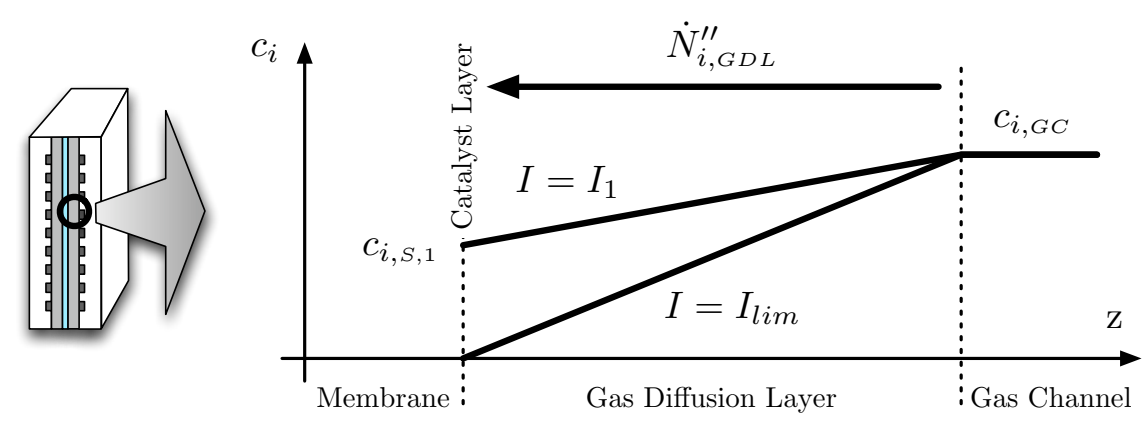

Figure A.1: Schematic representation of the limiting current density on a graph showing the reactant concentration $c_{i}$ as a function of the distance across the gas diffusion layer. 
The limiting current density $I_{\text {lim }}^{\prime \prime}$ is defined as the current density for which the reactant concentration at the reaction surface drops to zero (see Fig. A.1). (A.19) can therefore be rewritten as:

$$
I_{\text {limit }}^{\prime \prime}=n F D_{e q} \frac{c_{G C}}{\delta_{G D L}}
$$

Dividing (A.19) by (A.20) and substituting partial pressures for the gas concentrations yields:

$$
\frac{I^{\prime \prime}}{I_{\text {limit }}^{\prime \prime}}=1-\frac{p_{i, S}}{p_{i, G C}}
$$

Since the only reaction product is liquid water whose partial pressure remains unchanged, (A.21) can be substituted into (2.12).

$$
\eta_{d}=-\frac{\mathcal{R} T}{n F} \ln \left(1-\frac{I^{\prime \prime}}{I_{\text {limit }}^{\prime \prime}}\right)
$$

This represents a simple expression for the diffusion overpotential as a function of the limiting current density of the considered cell. However, since the limiting current density is not a constant for a given cell, but depends on the reactant concentration in the gas channel, such a description is only accurate for a given set of operating parameters and is therefore less useful for modelling a cell under varying operating conditions.

\section{A.4. Derivation of the Theoretical lonic Membrane Conductivity}

Ion transport along with water transport in the membrane can be described from basic transport equations. However, certain assumptions and simplifications have to be made for those equations to be applicable. These are: (a) pseudo two-phase flow of protons and water (b) hydrated membrane (c) no chemical or electrochemical reactions (d) phase interaction limited to friction forces (e) constant pressure gradient for water (f) protons and water in solution in the membrane (assumes no interaction between the two phases which is not correct - has been corrected by [12] by adding a drag term) (g) uniform temperature distribution (h) homogeneous $\mathrm{SO}_{3}^{-}$ distribution

The overall flux of charged species can then be described by a form of the Nernst-Planck equation. As shown in [37], they state that protons can 
move by migration, diffusion and convection. Adapting their equation to one-dimensional proton transport in the z-direction yields:

$$
\dot{N}_{i}^{\prime \prime}=-z_{i} \frac{F}{R T} D_{i} c_{i} \frac{\partial \Phi}{\partial z}-D_{i} \frac{\partial c_{i}}{\partial z}+c_{i} v
$$

where current density is related to proton migration by Faraday's Law (2.24) as stated below:

$$
I_{i}^{\prime \prime}=F \sum_{i} z_{i} \dot{N}_{i}^{\prime \prime}
$$

Denoting the membrane species with the subscript letter $m$ and assuming no adsorption of pore-fluid ions on the membrane structure, electroneutrality can be expressed as:

$$
z_{m} c_{m}+\sum_{i} z_{i} c_{i}=0
$$

In the case of a PEM membrane, the only mobile ions are hydrogen ions (charge number $z_{H^{+}}=-1$ ) and (A.25) can be written as:

$$
c_{H^{+}}=\sum_{i} z_{i} c_{i}=z_{m} c_{m}
$$

Proton concentration in the membrane can thus be considered constant and equal to the fixed charge site $\left(\mathrm{SO}_{3}^{-}\right)$concentration (i.e. no ion transfer by diffusion), the second term of (A.23) vanishes and, including (A.24), it can be rewritten to yield:

$$
I_{i o n}^{\prime \prime}=-D_{H^{+}} c_{H^{+}} \frac{F^{2}}{R T} \frac{\partial \Phi}{\partial z}+c_{H^{+}} F v
$$

To simplify notations, the notation of [37] is adopted and a membrane conductivity $\kappa_{m}$ is defined as:

$$
\kappa_{m} \equiv \frac{F^{2}}{R T} D_{H^{+}} c_{H^{+}}
$$

Including (A.28), (A.27) can be solved for the potential gradient.

$$
\frac{\partial \Phi}{\partial z}=-\frac{I_{i o n}^{\prime \prime}}{\kappa_{m}}+\frac{F}{\kappa_{m}} c_{H^{+}} v
$$


The average speed of motion (i.e. convection term in (A.23)) of the charged pore fluid is governed by electric potential and pressure gradients and can be described by a form of Schlögl's equation [37]:

$$
v=\frac{k_{\Phi}}{\mu} z_{m} c_{m} F \frac{\partial \Phi}{\partial z}-\frac{k_{p}}{\mu}\left[\frac{\partial p}{\partial z}-\rho_{H}+g\right]
$$

As shown by [48], proton speed due to pressure forces and buoyancy (second term of (A.30)) can typically be neglected with respect to the electrical force. Substituting (A.25), (A.30) can be rewritten to yield ${ }^{2}$ :

$$
v=\frac{k_{\Phi}}{\mu} c_{H^{+}} F \frac{\partial \Phi}{\partial z}
$$

Substituting (A.31) into (A.29) then yields for the membrane phase potential variation:

$$
\frac{\partial \Phi}{\partial z}=-\frac{I_{i o n}^{\prime \prime}}{\kappa_{m}\left(1-\frac{F^{2} c_{H^{+}}^{2} k_{\Phi}}{\mu \kappa_{m}}\right)}
$$

The ionic membrane overpotential is the potential difference between the membrane-anode and membrane-cathode interface. For reasons of simplicity, a linear potential variation $\left(\frac{d \Phi}{d z}=\frac{\eta_{m}}{\delta_{m}}\right)$ in the membrane shall be assumed where the parameters in (A.32) are considered as effective average membrane properties ${ }^{3}$. This can then simply be written as:

$$
\eta_{m}=-\frac{I_{i o n}^{\prime \prime} \delta_{m}}{\kappa_{m}\left(1-\frac{F^{2} c_{H^{+}}^{2} k_{\Phi}}{\mu \kappa_{m}}\right)}=-\frac{I_{i o n}^{\prime \prime} \delta_{m}}{\sigma_{m}}
$$

Where $\sigma_{m}$ is defined as the overall membrane conductivity. Apart from physical constants or membrane specific parameters $\left(c_{H^{+}}^{2}\right)$, it depends on the following two variables:

\footnotetext{
${ }^{2}$ Also neglected here is the influence of the water molecules' motion on proton flux. In the case of water flux, this influence will be accounted for by a drag term [12].

${ }^{3}$ The evaluation of those effective average membrane parameters constitutes an important and difficult part in order for this simplification to be representative of real behaviour.
} 
Ionic Membrane Conductivity As can be seen in (A.28), the ionic membrane conductivity $\kappa_{m}$ is directly proportional to the proton diffusion coefficient $D_{H^{+}}{ }^{4}$. Values typically proposed for the ionic membrane conductivity are shown in Table A.1.

Electrokinetic Permeability The electrokinetic permeability is the governing parameter for electroosmotic processes and is used in (A.30) to describe the proton speed due to electrical forces. Typical values for $k_{\Phi}$ are reported in Table A.1.

\begin{tabular}{ccccc}
\hline $\begin{array}{c}D_{H^{+}} \\
{\left[\mathrm{m}^{2} / \mathrm{s}\right]}\end{array}$ & $\begin{array}{c}\kappa_{m} \\
{[S / m]}\end{array}$ & $\begin{array}{c}k_{\Phi} \\
{\left[\mathrm{m}^{2}\right]}\end{array}$ & $\begin{array}{c}T \\
{[\circ \mathrm{o}]}\end{array}$ & Source \\
\hline $4.5 \cdot 10^{-9}$ & 17 & $7.18 \cdot 10^{-20}$ & 80 & Bernardi et al. [13] \\
$5.6 \cdot 10^{-9}$ & 21 & $7.18 \cdot 10^{-20}$ & 95 & Bernardi et al. [13] \\
$4.5 \cdot 10^{-9}$ & 17 & $1.13 \cdot 10^{-19}$ & 80 & Bernardi et al. [37] \\
$4.5 \cdot 10^{-9}$ & $7^{5}$ & $1.13 \cdot 10^{-19}$ & 80 & Bernardi et al. [37] \\
$1.4 \cdot 10^{-9}$ & - & $1.20 \cdot 10^{-19}$ & 22 & Verbrugge et al. [66] \\
$1.4 \cdot 10^{-9}$ & - & $1.13 \cdot 10^{-19}$ & 22 & Verbrugge et al. [67] \\
$3.5 \cdot 10^{-10}$ & - & - & - & Verbrugge et al. [68] \\
\hline
\end{tabular}

Table A.1: Values reported in literature for ionic membrane conductivity, proton diffusion coefficient and electrokinetic permeability.

\footnotetext{
${ }^{4} F, R$ and $c_{H^{+}}$are assumed constant.
}

${ }^{5}$ Used to fit experimental data. 


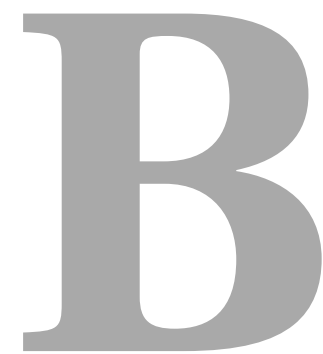

Definition of Mass Transfer Processes and Parameters 


\section{B.1. Air Composition}

Air is a mixture of several gases, the main components being Oxygen and Nitrogen. The contents of each for a standard atmosphere (i.e. $T_{0}=25^{\circ} \mathrm{C}$ and $p_{0}=101^{\prime} 325 \mathrm{~Pa}$ ) are given in Tab. B.1 [69]:

\begin{tabular}{lcc}
\hline Substance & $\begin{array}{c}p_{i} \\
{[\text { mbar }]}\end{array}$ & $\begin{array}{c}\chi_{i} \\
{[\text { mol i/mol } \mathrm{L}]}\end{array}$ \\
\hline $\mathrm{N}_{2}$ & 766.5 & 0.7565 \\
$\mathrm{O}_{2}$ & 205.6 & 0.2030 \\
$\mathrm{Ar}$ & 0.91 & 0.009 \\
$\mathrm{H}_{2} \mathrm{O}$ & 3.16 & 0.0312 \\
$\mathrm{CO}_{2}$ & 0.03 & 0.0003 \\
\hline
\end{tabular}

Table B.1: Partial pressures and molar content of air at standard conditions (i.e. $T_{0}=25^{\circ} \mathrm{C}$ and $p_{0}=101^{\prime} 325 \mathrm{~Pa}$ ).

In the context of this work a dry air composition of $80 \%$ Nitrogen and $20 \%$ Oxygen shall be assumed, thus neglecting any other constituents. Water content will be accounted for depending on the actual humidity conditions.

\section{B.2. Gas Solubility in Water}

Gas solubility in water is typically described by Henry's law [37] where the concentration of the dissolved gas is given as a function of partial gas pressure above the water surface.

$$
c_{d i s}=\frac{p_{A}}{H_{A}}
$$

For oxygen, values tabulated in [70] lead to the graph shown in Fig. B.1. Unfortunately, those values do not match with other references. Values from [71] show better coherence and have been curve-fitted (Fig. B.1 to yield:

$$
\ln \left(H_{\mathrm{O}_{2}-\mathrm{H}_{2} \mathrm{O}}\right)=-4.329 \cdot T^{2}+3847 \cdot T-6.821 \cdot 10^{5}
$$


For the membrane phase, Henry's constant $\left[\mathrm{atm} \cdot \mathrm{cm}^{3} / \mathrm{mol}\right]$ can be expressed as a function of temperature [37]:

$$
\ln \left(H_{O_{2}-M E M}\right)=-\frac{666}{T}+14.1
$$

Plotting the results of (B.3) over a temperature range between 0 and $100^{\circ} \mathrm{C}$ results in the Graph shown in Fig. B.1

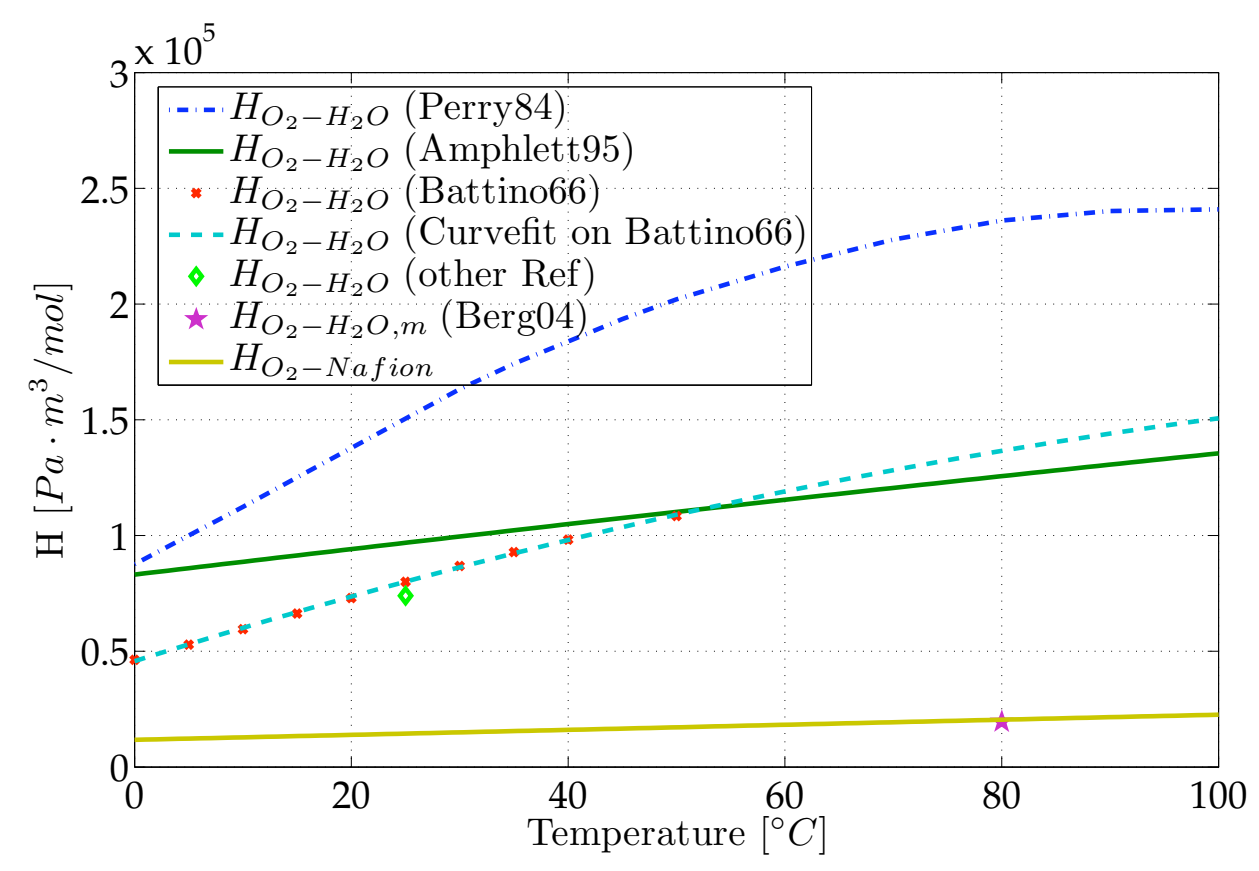

Figure B.1: Henry's constant as a function of temperature.

\section{B.3. Sherwood Type Mass Transfer}

In analogy to the Nusselt number in heat transfer (i.e ratio of total heat transfer to conductive heat transfer), the Sherwood number [72] (mass diffusivity/molar diffusivity) can be defined as:

$$
S h \equiv \frac{v_{d i f} L}{D_{A G}}
$$


where: $\quad D_{A G}=$ binary diffusion coefficient or dif- $\left[\mathrm{m}^{2} / \mathrm{s}\right]$ fusivity of component A with respect to gas mixture $\mathrm{G}$

$\begin{aligned} L & =\text { characteristic length } \\ v_{\text {dif }} & =\text { diffusion rate }\end{aligned}$

where $v_{d i f}[\mathrm{~m} / \mathrm{s}]$ is defined as a mass transfer coefficient. Multiplying $v_{d i f}$ by the corresponding surface area and the equivalent molar concentration difference of component A $\Delta c_{e q}\left[\mathrm{~mol} / \mathrm{m}^{3}\right]$ yields the effective molar mass transfer rate which can be written as:

$$
\dot{N}_{A}=S h \frac{D_{A G}}{L} A_{c} \Delta c_{e q}
$$

The equivalent molar concentration difference (or equivalent temperature difference for heat transfer problems) can be expressed as an arithmetic mean (B.6) for a constant molar flux $\dot{N}_{A}^{\prime \prime}(x, y)=$ const and for a constant exchange surface concentration $c_{e x}(x, y)=$ const as a logarithmic mean (B.7).

$$
\begin{aligned}
\Delta c_{\text {ar }} & =\frac{\Delta c_{\text {out }}-\Delta c_{\text {in }}}{2} \\
\Delta c_{\text {lm }} & =\frac{\Delta c_{\text {out }}-\Delta c_{\text {in }}}{\ln \frac{\Delta c_{\text {out }}}{\Delta c_{\text {in }}}}
\end{aligned}
$$

where $\Delta c$ is defined as the difference between the mean stream concentration and the concentration at the exchange surface.

\section{B.4. Diffusion}

Diffusion processes are concentration gradient driven phenomena. By their nature, they occur in multicomponent mixtures and do not contribute to an averaged overall movement of the fluid. If the individual species velocity due to diffusion is denoted by $v_{i}^{d i f}$ and its overall concentration (with respect to the total volume) by $\rho_{i}$, then it can be shown that:

$$
\sum_{i} v_{i}^{d i f} \rho_{i}=0
$$


(B.8) can be shown do result directly from the ideal gas law for a stagnant fluid. In fact, for a stagnant fluid, Darcy's Law (Schlögl's equation (A.30) for uncharged species) states that the pressure distribution is uniform. Therfore, overall gas concentration is invariable and the flow rates due to diffusion need to balance out.

Diffusional processes in one dimensional form, can be shown to obey a law of the form:

$$
\dot{N}_{A}^{\prime \prime}=c_{G} D_{A B} \frac{\partial \chi_{A}}{\partial z}=\left.D_{e q} \frac{\partial c_{A}}{\partial z}\right|_{\text {for } c_{G}=\text { const }}
$$

that is generally known as Fick's law. Note that expression B.9 is only valid in a reference frame moving with the average fluid velocity. For a stationary reference frame, appropriate corrections can be applied [57] to yield:

$$
\dot{N}_{A}^{\prime \prime}=-c D_{A B} \frac{\partial \chi_{A}}{\partial z}+\chi_{A}\left(\dot{N}_{A}^{\prime \prime}+\dot{N}_{B}^{\prime \prime}\right)
$$

For a stationary diffusion media (i.e. no bulk gas flow in the diffusion direction), (B.10) reduces to (B.9).

For multicomponent diffusion (n components), (B.9) can be expressed in the form of the Stefan Maxwell equation:

$$
\nabla \chi_{i}=\sum_{j=1}^{n} \frac{\mathcal{R} T}{p D_{i, j}}\left(\chi_{i} \dot{N}_{j}^{\prime \prime}-\chi_{j} \dot{N}_{i}^{\prime \prime}\right)
$$

\section{B.4.1. Binary Diffusion Coefficients}

The binary diffusion coefficients $D_{\mathrm{O}_{2}-\mathrm{N}_{2}}$ and $\mathrm{D}_{\mathrm{O}_{2}-\mathrm{H}_{2} \mathrm{O}}$ are determined from the Slattery-Bird equation (for binary diffusion coefficients of components $\mathrm{A}$ and $\mathrm{B})$ as outlined in [37]:

$$
\begin{aligned}
p D_{A B}= & a\left(\frac{T}{\sqrt{T_{c A} T_{c B}}}\right)^{b}\left(p_{c A} p_{c B}\right)^{1 / 3} \\
& \left(T_{c A} T_{c B}\right)^{5 / 12}\left(\frac{1}{\mathcal{M}_{A}}+\frac{1}{\mathcal{M}_{B}}\right)^{1 / 2}
\end{aligned}
$$




$$
\begin{aligned}
& \text { where: } \quad D_{A B}=\text { binary diffusion coefficient com- }\left[\mathrm{cm}^{2} / \mathrm{s}\right] \\
& \text { ponent A with respect to compon- } \\
& \text { ent B } \\
& p=\text { pressure } \\
& a, b=\mathrm{a}=0.0002745, \quad \mathrm{~b}=1.832 \quad \text { for } \\
& \mathrm{N}_{2}, \mathrm{O}_{2}, \mathrm{H}_{2} \\
& a, b=\mathrm{a}=0.000364, \mathrm{~b}=2.334 \text { for water va- } \quad[-] \\
& \text { pour } \\
& T_{c A}, T_{c B}=\text { critical temperatures of compon- } \quad[K] \\
& \text { ents A and B } \\
& p_{c A}, p_{c B}=\text { critical pressures of components } \quad[\mathrm{atm}] \\
& \mathrm{A} \text { and } \mathrm{B} \\
& \mathcal{M}_{a}, \mathcal{M}_{b}=\text { molecular weight of components }[\mathrm{g} / \mathrm{mol}] \\
& \mathrm{A} \text { and } \mathrm{B}
\end{aligned}
$$

This can be rewritten much simpler by regrouping all constants to yield:

$$
D_{A B}=k_{A B} \frac{T^{b_{A B}}}{p}
$$

\section{B.4.2. Mixture Diffusion Coefficients}

Based on the molar fractions of the constituents, an overall Diffusion coefficient of a species A within the gas mixture $\mathrm{G}$ of species $\mathrm{A}, \mathrm{B}$ and $\mathrm{C}$ can be derived according to:

$$
D_{A G}=\frac{1-\chi_{A}}{\frac{\chi_{B}}{D_{A B}}+\frac{\chi_{C}}{D_{A C}}}
$$

Introducing (B.13) yields:

$$
D_{A G}=\frac{1}{p} \frac{1-\chi_{A}}{\frac{\chi_{B}}{k_{A B} T^{b_{A B}}}+\frac{\chi_{C}}{k_{A C} T^{b_{A C}}}}
$$

The oxygen diffusivity - pressure product $p \cdot D_{\mathrm{O}_{2} L}$ has been plotted in Figure B.2 for humid air. It is shown as a function of the inlet water molar fraction $\chi_{\mathrm{H}_{2} \mathrm{O}, \mathrm{c}}^{i n}$, the water transfer coefficient $\alpha$ and the stoichiometric air flow rate $\lambda_{\mathrm{O}_{2}}$.

Note the almost constant pressure - diffusivity product apart from when $\alpha$ tends to large negative values and $\chi_{H_{2} O, c}^{i n}$ tends to zero, when a drop in 


$$
\lambda_{O_{2}}=1[-]
$$
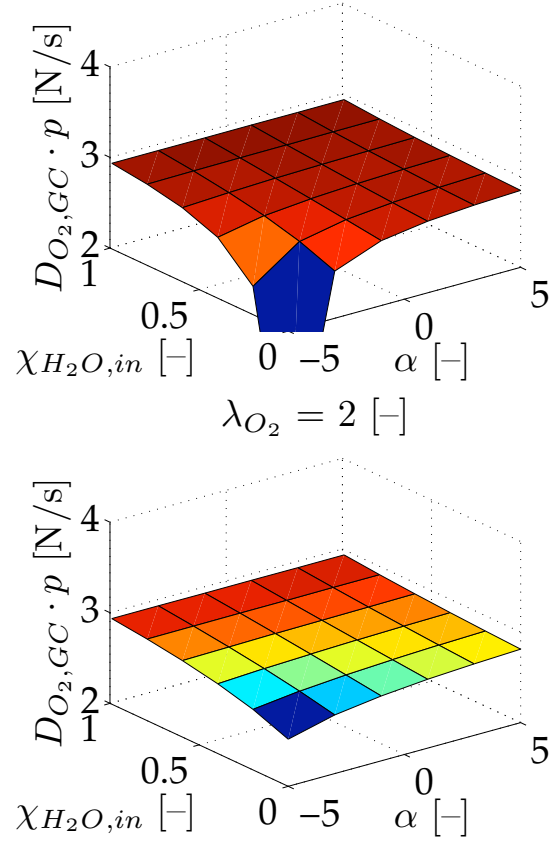

$$
\lambda_{O_{2}}=1.5[-]
$$
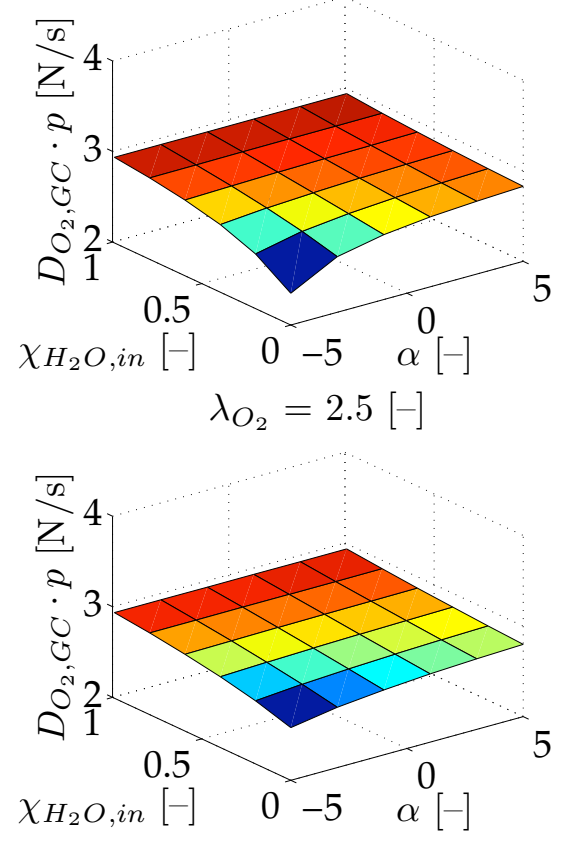

Figure B.2: Oxygen Diffusivity - Pressure product $p \cdot D_{O_{2} L}$ for oxygen diffusion in humid air as a function of inlet water molar fraction, water transfer coefficient and stoichiometric air ratio at a temperature of $70^{\circ} \mathrm{C}$.

the pressure - diffusivity product is observed. This drop is more pronounced the lower the stoichiometric air flow rate $\lambda_{O_{2}}$ and the higher the operating temperature.

\section{B.4.3. Diffusion in Porous Media}

The effective diffusion coefficient needs to be adjusted with a Bruggemann correction factor to account for the porous nature of the diffusion media REG(typically oxygen or water vapour diffusion in the porous GDL):

$$
D_{A G}^{R E G}=\phi^{3 / 2} D_{A G}
$$


where:

$$
\begin{aligned}
& \begin{array}{rlr}
\phi & =\text { porous media void fraction } & {[-]} \\
D_{A G}^{R E G} & =\text { effective diffusion coefficient of } & {\left[\mathrm{m}^{2} / \mathrm{s}\right]}
\end{array} \\
& \text { component A } \\
& D_{A G}=\text { diffusion coefficient of component }\left[\mathrm{m}^{2} / \mathrm{s}\right] \\
& \text { A within gas mixture } \mathrm{G}
\end{aligned}
$$


Analytical Solution for Water Transfer 


\section{C.1. Analytical Solution for Water Transport in a Fuel Cell}

The governing equations for water transport in a fuel cell (see section 2.5) can be simplified by introducing the following substitutions.

$$
\begin{gathered}
a=0 \\
b=14 \\
d_{1}=\frac{\left.D_{H_{2} O, M E M}\right|_{T=303 K}}{\exp \left(\frac{d_{5}}{303}\right)} \\
d_{5}=2436 \\
d_{6}=d_{1} \cdot \exp \left(\frac{d_{5}}{T}\right) \\
e=\frac{2.5}{22} \frac{I^{\prime \prime}}{F} \\
\frac{\rho_{M E M}}{E W_{M E M}} \frac{k_{p}}{\mu_{H_{2} O} O} \frac{\Delta p}{\delta_{M E M}} \\
k=\frac{1}{2} \frac{I^{\prime \prime}}{2 F} \frac{E W}{\gamma \rho} \\
m=\frac{\chi_{H_{2} O, c}^{i n}}{1-\chi_{H_{2} O, c}^{i n}} 5 \lambda_{O_{2}} \\
\chi_{H_{2} O, a}^{i n} \lambda_{H_{2}} \\
1-\chi_{H_{2} O, a}^{i n} \\
p=5 \lambda_{O_{2}} \\
q=\lambda_{H_{2}}
\end{gathered}
$$


The average molar water fraction in the cathode 2.79 and anode 2.80 gas channel then become:

$$
\begin{gathered}
\bar{\chi}_{H_{2} O, C G C}=\frac{m+\alpha+1}{m+p+\alpha+0.5} \\
\bar{\chi}_{H_{2} O, A G C}=\frac{n-0.5 \alpha}{n+q-0.5 \alpha-0.5}
\end{gathered}
$$

The governing equation for the overall water transport in the membrane (2.103) can be rewritten to yield:

$$
\alpha \frac{I^{\prime \prime}}{2 F}\left(1+\frac{d_{6}}{\delta_{m}} \frac{E W_{m}}{\gamma \rho_{m}}\right)=\left(e+f-\frac{2 d_{6}}{\delta_{m}}\right) \bar{\xi}+\frac{D_{H_{2} O, M E M}}{\delta_{m}} \xi_{a}^{*}
$$

In another round of substitutions, the following are introduced:

$$
\begin{gathered}
s=\frac{I^{\prime \prime}}{2 F}\left(1+\frac{d_{6}}{\delta_{m}} \frac{E W_{m}}{\gamma \rho_{m}}\right) \\
t=\left(e+f-\frac{2 d_{6}}{\delta_{m}}\right) \\
u=\frac{D_{H_{2} O, M E M}}{\delta_{m}}
\end{gathered}
$$

All the above substitutions and simplifications then yield a system of three equations for the unknowns $\xi_{a}^{*}, \bar{\xi}$ and $\alpha$ :

$$
\begin{gathered}
\xi_{a}^{*}=a+b \frac{n-0.5 \alpha}{q+n-0.5 \alpha-0.5} \\
\bar{\xi}=k+a+\frac{1}{2}\left[\frac{m+\alpha+1}{m+p+\alpha+0.5}+\frac{n-0.5 \alpha}{n+q-0.5 \alpha-0.5}\right] \\
\alpha=\frac{1}{s}\left(t \bar{\xi}+u \xi_{a}^{*}\right)
\end{gathered}
$$


Substituting (C.19) and (C.20) into (C.21) yields:

$$
\begin{aligned}
\alpha= & \frac{t}{s}\left(k+a+0.5 \frac{b(n-0.5 \alpha)}{n+q-0.5 \alpha-0.5}+0.5 \frac{b(m+\alpha+1)}{m+p+\alpha+0.5}\right) \\
& +\frac{u}{s}\left(a+\frac{b(n-0.5 \alpha)}{n+q-0.5 \alpha-0.5}\right)
\end{aligned}
$$

This can then be expanded to a cubic equation for the water transfer coefficient $\alpha$ as a function of the system parameters and operating conditions.

$$
0=a_{0}+a_{1} \alpha+a_{2} \alpha^{2}+a_{3} \alpha^{3}
$$

The coefficients in (2.109) are then given by the expressions below:

$$
\begin{aligned}
a_{0}= & -\frac{1}{2} t a p+\frac{1}{2} t k n+\frac{1}{2} u b n-\frac{1}{4} t b m-\frac{1}{4} t k-\frac{1}{4} t a-\frac{1}{4} u a \\
& -\frac{1}{2} t a m-\frac{1}{2} t k p-\frac{1}{2} t k m+\frac{1}{2} t k q+\frac{1}{2} t a q+\frac{1}{2} t b q \\
& +\frac{1}{2} t a n-\frac{1}{2} u a p-\frac{1}{2} u a m+\frac{1}{2} u a q+\frac{1}{2} u a n-\frac{1}{4} t b \\
& +\frac{3}{4} t b n+t k n m+\frac{1}{2} t b q m+\frac{1}{2} t b n p+t b n m+t a q p \\
& + \text { taqm }+ \text { tanp }+ \text { tanm }+ \text { tkqp }+t k q m+t k n p+u a n m \\
& +u a n p+u a q m+u a q p+u b n m+u b n p \\
a_{1}= & -\frac{1}{4} t b p-\frac{1}{2} u b m-\frac{1}{2} u b p-\frac{1}{2} s q+\frac{1}{2} s m-s n m-s q m \\
& +\frac{1}{4} s-\frac{1}{2} t a p+t k n+u b n-\frac{1}{2} t b m+\frac{1}{2} s p-s n p-\frac{1}{2} s n \\
& -s q p-\frac{1}{4} u b-\frac{3}{4} t k-\frac{3}{4} t a-\frac{3}{4} u a-\frac{1}{2} t a m-\frac{1}{2} t k p \\
& -\frac{1}{2} t k m+t k q+t a q+\frac{1}{2} t b q+t a n-\frac{1}{2} u a p-\frac{1}{2} u a m \\
& +u a q+u a n-\frac{5}{8} t b+t b n
\end{aligned}
$$




$$
\begin{aligned}
a_{2}= & \frac{3}{4} s-s q-s n-\frac{1}{2} t a-\frac{1}{2} t k-\frac{1}{2} u b-\frac{1}{2} u a+\frac{1}{2} s m \\
& +\frac{1}{2} s p-\frac{1}{2} t b
\end{aligned}
$$

\section{C.2. Cubic Formula}

This section outlines the analytical solution for the equation governing the water transport across the cell (C.23). The material presented is a résumé of the corresponding article in [73]. It outlines some of the general solutions to the standard cubic equation. Demonstrations and proofs have been neglected and only the solutions are presented - refer to [73] for more $\operatorname{details}^{1}$.

\section{C.2.1. General Solution}

A general cubic equation can be expressed as:

$$
z^{3}+a_{2} z^{2}+a_{1} z+a_{0}=0
$$

This can be transformed into

$$
x^{3}+3 Q x-2 R=0
$$

by substituting

$$
\begin{gathered}
x \equiv z+\frac{1}{3} a_{2} \\
Q \equiv \frac{3 a_{1}-a_{2}^{2}}{9} \\
R \equiv \frac{9 a_{2} a_{1}-27 a_{0}-2 a_{2}^{3}}{54}
\end{gathered}
$$

\footnotetext{
${ }^{1}$ There seems to be a problem with the way $T$ is defined in [73] - the formula does not produce correct results. The definition of $T$ presented in this section corrects the problem.
} 
Also defining

$$
\begin{aligned}
D & \equiv Q^{3}+R^{2} \\
S & \equiv \sqrt[3]{R+\sqrt{D}} \\
T & \equiv \frac{-Q}{\sqrt[3]{R+\sqrt{D}}}
\end{aligned}
$$

allows to express a general solution to (C.28) known as Cardano's formula:

$$
\begin{gathered}
z_{1}=-\frac{1}{3} a_{2}+(S+T) \\
z_{2}=-\frac{1}{3} a_{2}-\frac{1}{2}(S+T)+\frac{1}{2} i \sqrt{3}(S-T) \\
z_{3}=-\frac{1}{3} a_{2}-\frac{1}{2}(S+T)-\frac{1}{2} i \sqrt{3}(S-T)
\end{gathered}
$$

The number of complex roots is determined by the value of the polynomial discriminant $D$. If $D>0$, one root is real and two are complex conjugates, if $D=0$, all roots are real and at least two are equal and if $D<0$, all roots are real and unequal.

\section{C.2.2. Solution for Real Roots}

Alternative approaches are possible if the goal is to just determine the real roots of the equation.

\section{Real Roots for $\mathrm{D}<0$}

If the polynomial discriminant $D<0$, the solutions are given by:

$$
\begin{gathered}
z_{1}=2 \sqrt{-Q} \cos \left(\frac{\Theta}{3}\right)-\frac{1}{3} a_{2} \\
z_{2}=2 \sqrt{-Q} \cos \left(\frac{\Theta+2 \pi}{3}\right)-\frac{1}{3} a_{2} \\
z_{3}=2 \sqrt{-Q} \cos \left(\frac{\Theta+4 \pi}{3}\right)-\frac{1}{3} a_{2}
\end{gathered}
$$


where $\Theta$ is defined as

$$
\Theta \equiv \cos ^{-1}\left(\frac{R}{\sqrt{-Q^{3}}}\right) f
$$

\section{Real Roots for any value of $D$}

In this approach, the cubic equation (C.28) is transformed in to the standard form of Vieta (similar to (C.29))

$$
x^{3}+p x=q
$$

where $p=3 Q$ and $q=2 R$. The solutions are then given by:

$$
z_{i}=x_{i}-\frac{1}{3} a_{2}
$$

where $x_{i}$ and $y_{i}$ are given by

$$
\begin{gathered}
\qquad x_{i}=2 \sqrt{\frac{|p|}{3}} y_{i}-\frac{1}{3} a_{2} \\
y= \begin{cases}\cosh \left(\frac{1}{3} \cosh ^{-1} C\right) & \text { for } C \geq 1 \\
-\cosh \left(\frac{1}{3} \cosh ^{-1}|C|\right) & \text { for } C \leq-1 \\
\cos \left(\frac{1}{3} \cos ^{-1} C\right)[\text { three solutions }] & \text { for }|C|<1\end{cases}
\end{gathered}
$$

and $C$ is defined as

$$
C \equiv \frac{1}{2} q\left(\frac{3}{|p|}\right)^{\frac{3}{2}}
$$





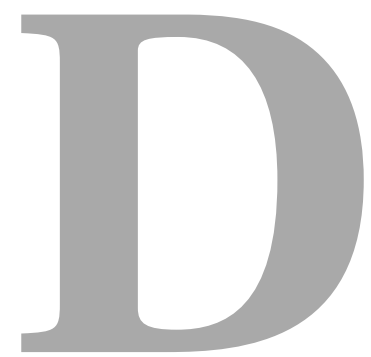

State Space Notation 


\section{D.1. Introduction}

This chapter will give a brief introduction to state space notation. Additionally, the notational conventions are explained and illustrated.

\section{D.2. Representation}

Most physical systems can be described as a combination of differential and algebraic equations that can be expressed as [74]:

$$
\begin{gathered}
\dot{\mathbf{x}}(t)=\left[\begin{array}{c}
f_{1}[\mathbf{x}(t), \mathbf{u}(t), t] \\
f_{2}[\mathbf{x}(t), \mathbf{u}(t), t] \\
\vdots \\
f_{n}[\mathbf{x}(t), \mathbf{u}(t), t]
\end{array}\right] \\
\mathbf{y}(t)=\left[\begin{array}{c}
g_{1}[\mathbf{x}(t), \mathbf{u}(t), t] \\
g_{2}[\mathbf{x}(t), \mathbf{u}(t), t] \\
\vdots \\
g_{p}[\mathbf{x}(t), \mathbf{u}(t), t]
\end{array}\right]
\end{gathered}
$$

where $\mathbf{x}(t), \dot{\mathbf{x}}(t), \mathbf{u}(t)$ and $\mathbf{y}(t)$ are the state vector, the state vector's time derivative, the input vector and the output vector respectively. They can also be written as:

$$
\begin{gathered}
\mathbf{x}(t)=\left[\begin{array}{c}
x_{1}(t) \\
x_{2}(t) \\
\vdots \\
x_{n}(t)
\end{array}\right] ; \quad \dot{\mathbf{x}}(t)=\left[\begin{array}{c}
\dot{x}_{1}(t) \\
\dot{x}_{2}(t) \\
\vdots \\
\dot{x}_{n}(t)
\end{array}\right] \\
\mathbf{u}(t)=\left[\begin{array}{c}
u_{1}(t) \\
u_{2}(t) \\
\vdots \\
u_{r}(t)
\end{array}\right] ; \quad \mathbf{y}(t)=\left[\begin{array}{c}
y_{1}(t) \\
y_{2}(t) \\
\vdots \\
y_{p}(t)
\end{array}\right]
\end{gathered}
$$


If the system is linear, the above equations can be represented in matrix form which yields a simple and convenient expression. For an analog system, this is typically written $a^{1}$ :

$$
\begin{aligned}
& \dot{\mathbf{x}}(t)=[\mathbf{A}]_{n \times n} \mathbf{x}(t)+[\mathbf{B}]_{n \times r} \mathbf{u}(t) \\
& \mathbf{y}(t)=[\mathbf{C}]_{p \times n} \mathbf{x}(t)+[\mathbf{D}]_{p \times r} \mathbf{u}(t)
\end{aligned}
$$

For the case of a discrete time system, this is usually expressed as:

$$
\begin{gathered}
\mathbf{x}(k+1)=[\boldsymbol{\Phi}]_{n \times n} \mathbf{x}(k)+[\boldsymbol{\Gamma}]_{n \times r} \mathbf{u}(k) \\
\mathbf{y}(k)=[\mathbf{C}]_{p \times n} \mathbf{x}(k)+[\mathbf{D}]_{p \times r} \mathbf{u}(k)
\end{gathered}
$$

\section{D.3. Discretisation}

In order to transform an analog system into discrete notation (typically to design a microcontroller or DSP based control system), $\boldsymbol{\Phi}$ and $\boldsymbol{\Gamma}$ are given as a function of the analog state space matrices $\mathbf{A}$ and $\mathbf{B}$ with $h$ as the sampling period:

$$
\begin{gathered}
\mathbf{\Phi}=e^{\mathbf{A} h} \\
\boldsymbol{\Gamma}=\int_{0}^{h} e^{\mathbf{A} \eta} d \eta \mathbf{B}
\end{gathered}
$$

\footnotetext{
${ }^{1}$ Shown here is a time invariant system where the matrices $\mathbf{A}, \mathbf{B}, \mathbf{C}$ and $\mathbf{D}$ do not depend on time.
} 


\section{D.4. LINEARISATION}

Since (D.9) and (D.10) are difficult to evaluate directly through the above equations, they can be approximated through the following set of equations:

$$
\begin{aligned}
\boldsymbol{\Phi} & =\mathbf{I}+\mathbf{A} h \boldsymbol{\Psi} \\
\boldsymbol{\Gamma} & =\mathbf{\Psi} h \mathbf{B}=\mathbf{A}^{-1}(\boldsymbol{\Phi}-\mathbf{I}) \mathbf{B} \\
\boldsymbol{\Psi} & =\sum_{i=0}^{\inf } \frac{\mathbf{A}^{i} h^{i}}{(i+1) !}
\end{aligned}
$$

\section{D.4. Linearisation}

A system that is described through a set of nonlinear state space equations (D.1) and (D.2) can be linearised for small variations around an operating point. In such a case, the actual value of the state-, input- or output vector is described as $\mathbf{x}, \mathbf{u}$ and $\mathbf{y}$. The nominal operating point is noted as $\overline{\mathbf{x}}$, $\overline{\mathbf{u}}$ and $\overline{\mathbf{y}}$ and deviations from that operating point are indicated by the

character such that:

$$
\begin{aligned}
\tilde{\mathbf{x}} & =\mathbf{x}-\overline{\mathbf{x}} \\
\tilde{\mathbf{u}} & =\mathbf{u}-\overline{\mathbf{u}} \\
\tilde{\mathbf{y}} & =\mathbf{y}-\overline{\mathbf{y}}
\end{aligned}
$$

Deriving (D.12) yields:

$$
\dot{\mathbf{x}}(t)=\dot{\overline{\mathbf{x}}}(t)+\dot{\tilde{\mathbf{x}}}(t)
$$

The linearised state space equation for the system can then be written as:

$$
\begin{aligned}
& \dot{\tilde{\mathbf{x}}}(t)=\mathbf{A}(t) \tilde{\mathbf{x}}(t)+\mathbf{B}(t) \tilde{\mathbf{u}}(t) \\
& \tilde{\mathbf{y}}(t)=\mathbf{C}(t) \tilde{\mathbf{x}}(t)+\mathbf{D}(t) \tilde{\mathbf{u}}(t)
\end{aligned}
$$


where $\mathbf{A}, \mathbf{B}, \mathbf{C}$ and $\mathbf{D}$ are the Jacobian matrices of the system with respect to the states and system inputs. They are given by:

$$
\begin{aligned}
\mathbf{A}(t)= & {\left.\left[\begin{array}{cccc}
\frac{\partial f_{1}}{\partial x_{1}} & \frac{\partial f_{1}}{\partial x_{2}} & \ldots & \frac{\partial f_{1}}{\partial x_{n}} \\
\frac{\partial f_{2}}{\partial x_{1}} & \frac{\partial f_{2}}{\partial x_{2}} & \cdots & \frac{\partial f_{2}}{\partial x_{n}} \\
\vdots & \vdots & \ddots & \vdots \\
\frac{\partial f_{n}}{\partial x_{1}} & \frac{\partial f_{n}}{\partial x_{2}} & \cdots & \frac{\partial f_{n}}{\partial x_{n}}
\end{array}\right]\right|_{\overline{\mathbf{x}}(t), \overline{\mathbf{u}}(t)} } \\
\mathbf{B}(t)= & {\left.\left[\begin{array}{cccc}
\frac{\partial f_{1}}{\partial u_{1}} & \frac{\partial f_{1}}{\partial u_{2}} & \cdots & \frac{\partial f_{1}}{\partial u_{r}} \\
\frac{\partial f_{2}}{\partial u_{1}} & \frac{\partial f_{2}}{\partial u_{2}} & \cdots & \frac{\partial f_{2}}{\partial u_{r}} \\
\vdots & \vdots & \ddots & \vdots \\
\frac{\partial f_{n}}{\partial u_{1}} & \frac{\partial f_{n}}{\partial u_{2}} & \cdots & \frac{\partial f_{n}}{\partial u_{r}}
\end{array}\right]\right|_{\overline{\mathbf{x}}(t), \overline{\mathbf{u}}(t)} } \\
\mathbf{C}(t)= & {\left.\left[\begin{array}{cccc}
\frac{\partial g_{1}}{\partial x_{1}} & \frac{\partial g_{1}}{\partial x_{2}} & \cdots & \frac{\partial g_{1}}{\partial x_{n}} \\
\frac{\partial g_{2}}{\partial x_{1}} & \frac{\partial g_{2}}{\partial x_{2}} & \cdots & \frac{\partial g_{2}}{\partial x_{n}} \\
\vdots & \vdots & \ddots & \vdots \\
\frac{\partial g_{p}}{\partial x_{1}} & \frac{\partial g_{p}}{\partial x_{2}} & \cdots & \frac{\partial g_{p}}{\partial x_{n}}
\end{array}\right]\right|_{\overline{\mathbf{x}}(t), \overline{\mathbf{u}}(t)} } \\
\mathbf{D}(t)= & {\left.\left[\begin{array}{cccc}
\frac{\partial g_{1}}{\partial u_{1}} & \frac{\partial g_{1}}{\partial u_{2}} & \cdots & \frac{\partial g_{1}}{\partial u_{r}} \\
\frac{\partial g_{2}}{\partial u_{1}} & \frac{\partial g_{2}}{\partial u_{2}} & \cdots & \frac{\partial g_{2}}{\partial u_{r}} \\
\vdots & \vdots & \ddots & \vdots \\
\frac{\partial g_{p}}{\partial u_{1}} & \frac{\partial g_{p}}{\partial u_{2}} & \cdots & \frac{\partial g_{p}}{\partial u_{r}}
\end{array}\right]\right|_{\overline{\mathbf{x}}(t), \overline{\mathbf{u}}(t)} }
\end{aligned}
$$

The same procedure holds of course for a system in discrete representation where $\mathbf{A}$ and $\mathbf{B}$ will simply be replaced by $\boldsymbol{\Phi}$ and $\boldsymbol{\Gamma}$. 


\section{D.5. Controllability and Observability}

A state space system is said to be controllable iff ${ }^{2}$ the controllability matrix $\mathbf{G}$ has full rank $n$, i.e.

$$
\operatorname{rank}(\mathbf{G})=\operatorname{rank}\left[\begin{array}{llll}
\mathbf{B} & \mathbf{A B} & \cdots & \mathbf{A}^{n-1} \mathbf{B}
\end{array}\right]_{n \times n r}=n
$$

A state space system is said to be observable iff ${ }^{2}$ the observabiltiy matrix $\mathbf{Q}$ has full rank $n$, i.e.

$$
\operatorname{rank}(\mathbf{Q})=\operatorname{rank}\left[\begin{array}{c}
\mathbf{C} \\
\mathbf{C A} \\
\vdots \\
\mathbf{C A}^{n-1}
\end{array}\right]_{n p \times n}=n
$$

\section{D.6. State Space Averaging}

State space averaging is a technique developed for modelling power electronic converters. Typically, in such converters power is transferred by periodically (at a duty cycle $d$ ) switching a set of power electronic switches from position 1 to position 2. While the switches are in position 1 , the system is described through a state space model $\left(\mathbf{A}_{1}, \mathbf{B}_{1}, \mathbf{C}_{1}, \mathbf{D}_{1}\right)$ and while they are in position 2 , the characteristic equations are given by $\left(\mathbf{A}_{2}, \mathbf{B}_{2}, \mathbf{C}_{2}, \mathbf{D}_{2}\right)$.

The state space averaging approach can be extended to any system that is characterised by a first state space model during $d \cdot T_{s}$ and by a second state space model during $(1-d) \cdot T_{s}\left(\right.$ where $T_{s}=\frac{1}{f}$ is the switching period), provided that:

1. The natural frequencies of the subsystems $\mathbf{A}_{1}$ and $\mathbf{A}_{2}$ are much smaller than the switching frequency $f$.

If such is the case, the state space equations can be written as:

$$
\dot{\tilde{\mathbf{x}}}(t)=\mathbf{A} \tilde{\mathbf{x}}(t)+\mathbf{B} \tilde{\mathbf{u}}(t)+\left[\left(\mathbf{A}_{1}-\mathbf{A}_{2}\right) \overline{\mathbf{x}}(t)+\left(\mathbf{B}_{1}-\mathbf{B}_{2}\right) \overline{\mathbf{u}}(t)\right] \tilde{d}(t)
$$

\footnotetext{
${ }^{2}$ Short form for "if and only if".
} 


$$
\tilde{\mathbf{y}}(t)=\mathbf{C} \tilde{\mathbf{x}}(t)+\mathbf{D} \tilde{\mathbf{u}}(t)+\left[\left(\mathbf{C}_{1}-\mathbf{C}_{2}\right) \overline{\mathbf{x}}(t)+\left(\mathbf{D}_{1}-\mathbf{D}_{2}\right) \overline{\mathbf{u}}(t)\right] \tilde{d}(t)
$$

where the averaged state space matrices are given by:

$$
\begin{aligned}
& \mathbf{A}=d \mathbf{A}_{1}+(1-d) \mathbf{A}_{2} \\
& \mathbf{B}=d \mathbf{B}_{1}+(1-d) \mathbf{B}_{2} \\
& \mathbf{C}=d \mathbf{C}_{1}+(1-d) \mathbf{C}_{2} \\
& \mathbf{D}=d \mathbf{D}_{1}+(1-d) \mathbf{D}_{2}
\end{aligned}
$$

This can be rewritten in the form of a standard state space equation with the variation from the nominal duty cycle $\tilde{\mathbf{d}}$ being added to the input vector $\tilde{\mathbf{u}}$.

$$
\begin{gathered}
\dot{\tilde{\mathbf{x}}}(t)=\mathbf{A} \tilde{\mathbf{x}}(t)+\left[\begin{array}{ccccc}
\mathbf{B}_{11} & \mathbf{B}_{12} & \ldots & \mathbf{B}_{1 r} & \mathbf{B}_{1(r+1)} \\
\mathbf{B}_{21} & \mathbf{B}_{22} & \ldots & \mathbf{B}_{2 r} & \mathbf{B}_{2(r+1)} \\
\vdots & \vdots & \ddots & \vdots & \vdots \\
\mathbf{B}_{n 1} & \mathbf{B}_{n 2} & \ldots & \mathbf{B}_{n r} & \mathbf{B}_{n(r+1)}
\end{array}\right]\left[\begin{array}{c}
\tilde{\mathbf{u}}_{1} \\
\tilde{\mathbf{u}}_{2} \\
\vdots \\
\tilde{\mathbf{u}}_{r} \\
\tilde{d}(t)
\end{array}\right] \\
\tilde{\mathbf{y}}(t)=\mathbf{C} \tilde{\mathbf{x}}(t)+\left[\begin{array}{ccccc}
\mathbf{D}_{11} & \mathbf{D}_{12} & \ldots & \mathbf{D}_{1 r} & \mathbf{D}_{1(r+1)} \\
\mathbf{D}_{21} & \mathbf{D}_{22} & \ldots & \mathbf{D}_{2 r} & \mathbf{D}_{2(r+1)} \\
\vdots & \vdots & \ddots & \vdots & \vdots \\
\mathbf{D}_{n 1} & \mathbf{D}_{p 2} & \ldots & \mathbf{D}_{p r} & \mathbf{D}_{p(r+1)}
\end{array}\right]\left[\begin{array}{c}
\tilde{\mathbf{u}}_{1} \\
\tilde{\mathbf{u}}_{2} \\
\vdots \\
\tilde{\mathbf{u}}_{r} \\
\tilde{d}(t)
\end{array}\right]
\end{gathered}
$$

The last columns of the extended $\mathbf{B}$ and $\mathbf{D}$ matrices are given by:

$$
\left[\begin{array}{c}
\mathbf{B}_{1(r+1)} \\
\mathbf{B}_{2(r+1)} \\
\vdots \\
\mathbf{B}_{n(r+1)}
\end{array}\right]=\left[\left(\mathbf{A}_{1}-\mathbf{A}_{2}\right) \overline{\mathbf{x}}(t)+\left(\mathbf{B}_{1}-\mathbf{B}_{2}\right) \overline{\mathbf{u}}(t)\right]
$$




$$
\left[\begin{array}{c}
\mathbf{D}_{1(r+1)} \\
\mathbf{D}_{2(r+1)} \\
\vdots \\
\mathbf{D}_{p(r+1)}
\end{array}\right]=\left[\left(\mathbf{C}_{1}-\mathbf{C}_{2}\right) \overline{\mathbf{x}}(t)+\left(\mathbf{D}_{1}-\mathbf{D}_{2}\right) \overline{\mathbf{u}}(t)\right]
$$




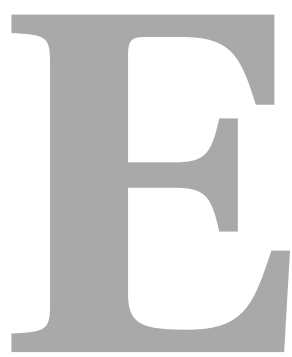

Matlab Implementation 


\section{E.1. Introduction}

The data structure in Appendix E.2 shows the variables that are available in Matlab after analysing the data from the fuel cell test bench.

Appendix E.3 lists the fuel cell specific parameters and universal constants that can be called by the modelling routines in Matlab.

For each variable, its values, units and labels can be called by the appropriate Matlab functions value, units and label respectively (see help on those functions for more details).

In order to update the tables in this document, run the m-files (in the listings directory of this document) data_structure.m and help_fc.m that have to be in the Listings directory for the main document (the LaTeX source for this document is in the chapters folder).

data_structure.m will generate two text files containing all the variables and constants used. Those text files are polled in order to generate the tables in chapter E.2 and E.3 when compiling this document.

\section{E.2. Data Structure}

\begin{tabular}{|c|c|c|}
\hline Variable & Label & Units \\
\hline year & Year & - \\
\hline month & Month & - \\
\hline day & Day & $d$ \\
\hline hour & Hour & $h$ \\
\hline minute & Minute & $\min$ \\
\hline second & Second & $s$ \\
\hline frequency & DAQ Frequency & $H z$ \\
\hline filter_time_const & Filter Time Constant & $s$ \\
\hline timestep & IV Timestep & $s$ \\
\hline points & Number of Samples & - \\
\hline time & Time & $s$ \\
\hline mes_hour & Hour $_{m e s}$ & $h$ \\
\hline mes_min & Minute $_{m e s}$ & $\min$ \\
\hline mes_sec & Second $_{m e s}$ & $s$ \\
\hline$v_{-} f \bar{c}$ & FC Voltage & $V$ \\
\hline $\mathrm{v}_{-}^{-}$air & Air Compressor Voltage & $V$ \\
\hline
\end{tabular}


continued from previous page...

\begin{tabular}{|c|c|c|}
\hline Variable & Label & Units \\
\hline $\mathrm{V} \quad \mathrm{cl}$ & CL Pump Voltage & $V$ \\
\hline$v_{-}^{-} \mathrm{h} 2$ & $H_{2}$ Pump Voltage & $V$ \\
\hline$i_{-}$fc & FC Current & $A$ \\
\hline$i^{-}$air & Air Compressor Current & $A$ \\
\hline i_cl & CL Pump Current & $A$ \\
\hline $\mathrm{i}^{-} \mathrm{h} 2$ & $H_{2}$ Pump Current & $A$ \\
\hline$\overline{\mathrm{m}}$-air & Air Massflow & slpm \\
\hline $\mathrm{h} \_$air_amb & Ambient Air Humidity & $\% R H$ \\
\hline $\mathrm{m} \_\mathrm{h} 2$ & $\mathrm{H}_{2}$ Massflow & slpm \\
\hline $\mathrm{p}$ _air & Air Pressure & $\operatorname{mbar}(g)$ \\
\hline $\mathrm{p} \_\mathrm{h} 2$ & $H_{2}$ Pressure & $\operatorname{mbar}(g)$ \\
\hline $\mathrm{p} \_\mathrm{amb}$ & Ambient Pressure & $\operatorname{mbar}(a)$ \\
\hline h_air_out & Air Humidity out & $\% R H$ \\
\hline $\mathrm{h}$ h $2^{-}$in & $H_{2}$ Humidity $_{i n}$ & $\% R H$ \\
\hline $\mathrm{a}-2 \_\overline{0} 0$ & Undefined & $V$ \\
\hline t_air_cond & Air $\mathrm{T}_{\text {cond }}$ & ${ }^{\circ} \mathrm{C}$ \\
\hline $\mathrm{t}$ _air_in & Air $\mathrm{T}_{i n}$ & ${ }^{\circ} \mathrm{C}$ \\
\hline t_air_out & Air $\mathrm{T}_{\text {out }}$ & ${ }^{\circ} \mathrm{C}$ \\
\hline t_air_dp & $\operatorname{Air} \mathrm{T}_{d p}$ & ${ }^{\circ} \mathrm{C}$ \\
\hline $\mathrm{t}$-h2_out & $H_{2} \mathrm{~T}_{\text {out }}$ & ${ }^{\circ} \mathrm{C}$ \\
\hline $\mathrm{t}$ - $\mathrm{h} 2$ - cond & $H_{2} \mathrm{~T}_{\text {cond }}$ & ${ }^{\circ} \mathrm{C}$ \\
\hline $\mathrm{t}_{-} \mathrm{cl}_{-}$in & $\mathrm{CL} \mathrm{T}_{i n}$ & ${ }^{\circ} \mathrm{C}$ \\
\hline t_cl_out & $\mathrm{CL} \mathrm{T}_{\text {out }}$ & ${ }^{\circ} \mathrm{C}$ \\
\hline t_cl_hx & $\mathrm{CL} \mathrm{T}_{H X}$ & ${ }^{\circ} \mathrm{C}$ \\
\hline $\mathrm{t}_{-} \mathrm{c} \overline{\mathrm{w}}_{-}$in & $\mathrm{CW} \mathrm{T}_{i n}$ & ${ }^{\circ} \mathrm{C}$ \\
\hline $\mathrm{t} \_\mathrm{h} 2$ in & $H_{2} \mathrm{~T}_{i n}$ & ${ }^{\circ} \mathrm{C}$ \\
\hline t_air_comp & Air $\mathrm{T}_{c o m p}$ & ${ }^{\circ} \mathrm{C}$ \\
\hline$t_{\text {_air_amb }}$ & Air $\mathrm{T}_{a m b}$ & ${ }^{\circ} \mathrm{C}$ \\
\hline$t_{-} 14$ & Undefined $\mathrm{T}_{14}$ & ${ }^{\circ} \mathrm{C}$ \\
\hline$t-15$ & Undefined $\mathrm{T}_{15}$ & ${ }^{\circ} \mathrm{C}$ \\
\hline $\mathrm{m}_{-} \mathrm{cl}$ & CL Massflow & slpm \\
\hline m_air_control & Setpoint Air Massflow & slpm \\
\hline $\mathrm{m}$-h2_control & Setpoint $H_{2}$ Massflow & slpm \\
\hline $\mathrm{m} \_\mathrm{cl} \_$control & Setpoint CL Massflow & slpm \\
\hline
\end{tabular}

continued on next page... 
continued from previous page...

\begin{tabular}{|c|c|c|}
\hline Variable & Label & Units \\
\hline $\mathrm{da} 4$ & Undefined $\mathrm{DA}_{4}$ & $V$ \\
\hline r_h2_cbp & $H_{2}$ Condenser Bypass & $1_{o n}, 0_{o f f}$ \\
\hline $\mathrm{r}$ h 2 - purge & $H_{2}$ Purge & $1_{o n}, 0_{o f f}$ \\
\hline r_cw_valve & CW Valve & $1_{\text {closed }}, 0_{\text {open }}$ \\
\hline r_cw_pump & CW Pump & $1_{\text {on }}, 0_{\text {off }}$ \\
\hline $\mathrm{r} \overline{5}$ & $\mathrm{R}_{5}$ & $1_{\text {on }}, 0_{\text {off }}$ \\
\hline r6 & $\mathrm{R}_{6}$ & $1_{\text {on }}, 0_{\text {off }}$ \\
\hline $\mathrm{v} \_$cell_1 & Cell Voltage 1 & $m V$ \\
\hline $\mathrm{v}$-cell_-2 & Cell Voltage 2 & $m V$ \\
\hline $\mathrm{v}$ - cell_ 3 & Cell Voltage 3 & $m V$ \\
\hline $\mathrm{v}$ _cell- 4 & Cell Voltage 4 & $m V$ \\
\hline $\mathrm{v}_{-}{ }_{-}$cell 5 & Cell Voltage 5 & $m V$ \\
\hline $\mathrm{v}$ _cell-6 6 & Cell Voltage 6 & $m V$ \\
\hline $\mathrm{v}$ - cell- 7 & Cell Voltage 7 & $m V$ \\
\hline power_fc & FC Power & $W$ \\
\hline power_air & Air Compressor Power & $W$ \\
\hline power_cl & CL Pump Power & $W$ \\
\hline power ${ }^{-}$h2 & $H_{2}$ Pump Power & $W$ \\
\hline lambda_air & $\lambda_{A i r}$ & - \\
\hline lambda_h2 & $\lambda_{H 2}$ & - \\
\hline eta_gross_hhv & $\eta_{H H V}$ (gross) & $\%$ \\
\hline eta_gross_lhv & $\eta_{L H V}$ (gross) & $\%$ \\
\hline eta_net_hhv & $\eta_{H H V}$ (net) & $\%$ \\
\hline eta_net_lhv & $\eta_{L H V}($ net $)$ & $\%$ \\
\hline spec power fc & Specific FC Power & $W / m^{2}$ \\
\hline spec $^{-} \mathrm{i}$ fc & Specific FC Current & $A / m^{2}$ \\
\hline v_cell__avg & Average Cell Voltage & $V$ \\
\hline $\mathrm{t}$ _air_avg & Average Air Temperature & ${ }^{\circ} \mathrm{C}$ \\
\hline $\mathrm{t}^{-} \mathrm{h} 2{ }^{-}$avg & Average Hydrogen Temperature & ${ }^{\circ} \mathrm{C}$ \\
\hline t_air_adm_avg & $\begin{array}{l}\text { Average Inlet Chamber Air } \\
\text { Temperature }\end{array}$ & ${ }^{\circ} \mathrm{C}$ \\
\hline$t_{\text {_ }} \mathrm{fc} \_$est & $\begin{array}{l}\text { Estimated Fuel Cell Temperat- } \\
\text { ure }\end{array}$ & ${ }^{\circ} \mathrm{C}$ \\
\hline t_cell_avg & $\begin{array}{l}\text { Estimated Average Fuel Cell } \\
\text { Temperature }\end{array}$ & ${ }^{\circ} \mathrm{C}$ \\
\hline
\end{tabular}

continued on next page... 
continued from previous page...

\begin{tabular}{llc}
\hline Variable & Label & Units \\
\hline p_air_avg & Average Cathode Pressure & $P a(a)$ \\
p_h2_avg & Average Anode Pressure & $P a(a)$ \\
t_h2_dp & Anode Inlet Dew Point Temper- & ${ }^{\circ} C$ \\
mol_frac_h2o_a_in & $\begin{array}{l}\text { Ature } \\
\text { tion }\end{array}$ & - \\
mol_frac_h2o_c_in & $\begin{array}{l}\text { Cathode Inlet Molar Water } \\
\text { Fraction } \\
\text { dc_cv }\end{array}$ & \begin{tabular}{l} 
Chill Water Valve Duty Cycle \\
\hline
\end{tabular} \\
\hline
\end{tabular}

\section{E.3. Constants}




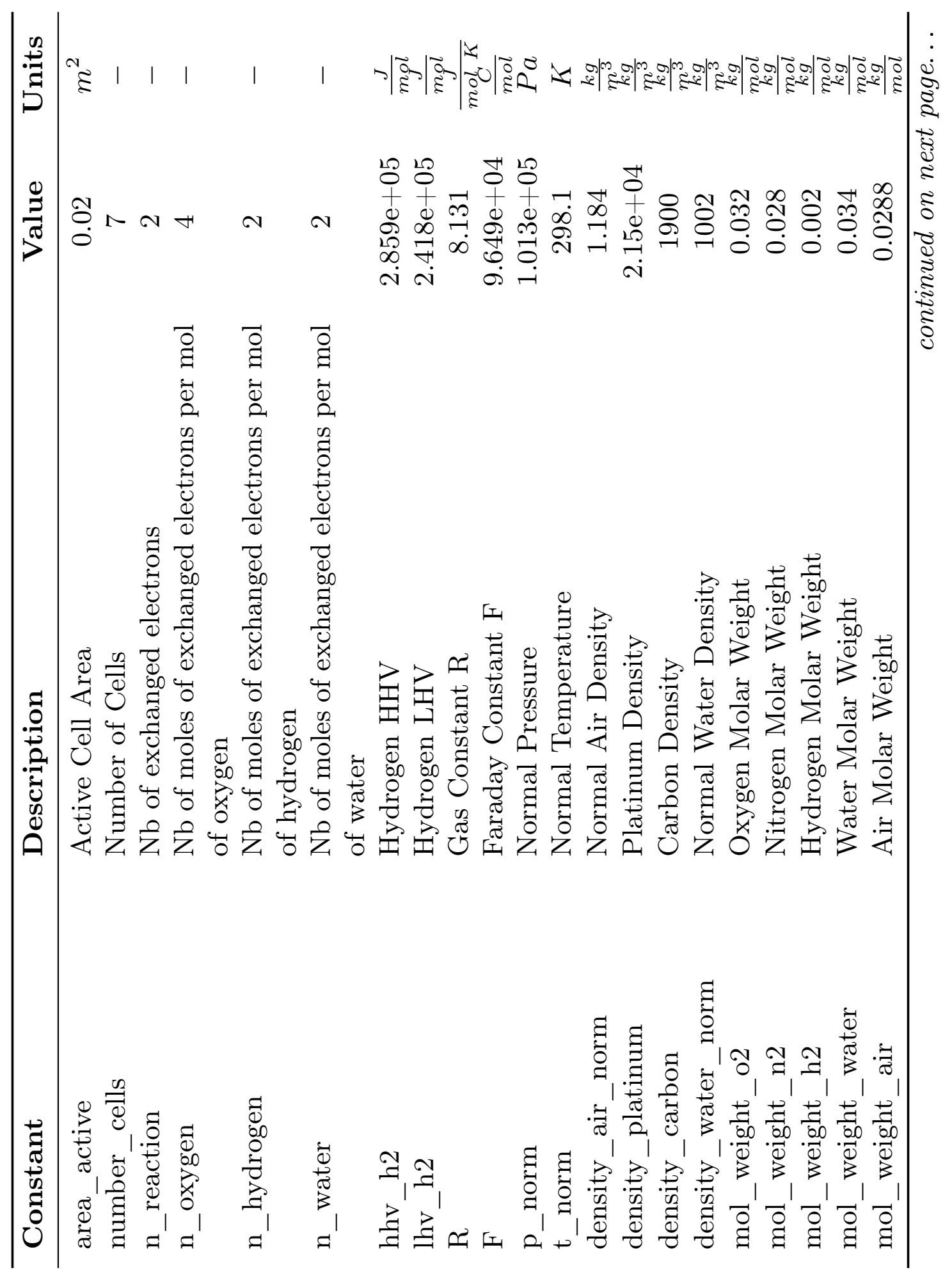




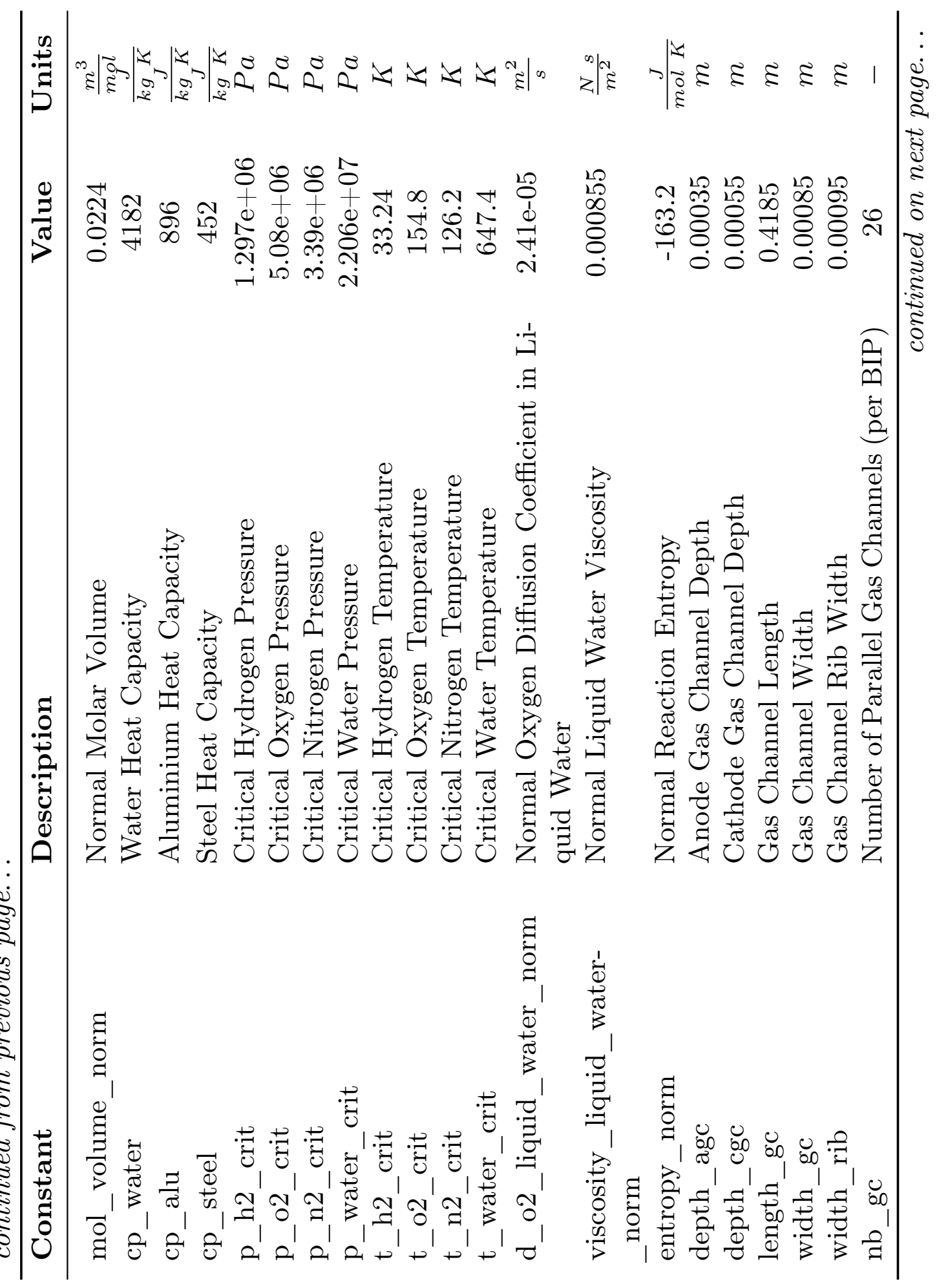




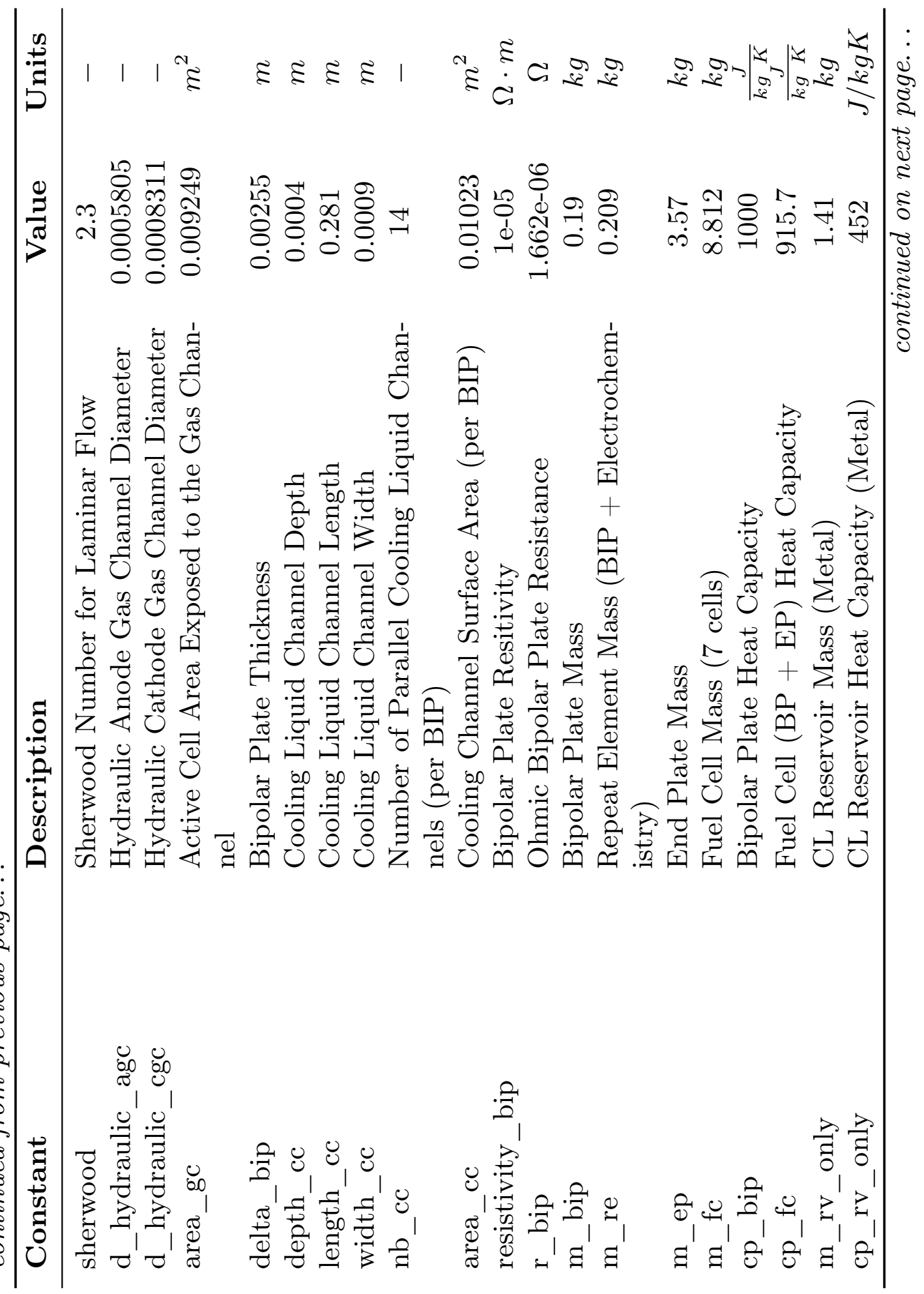




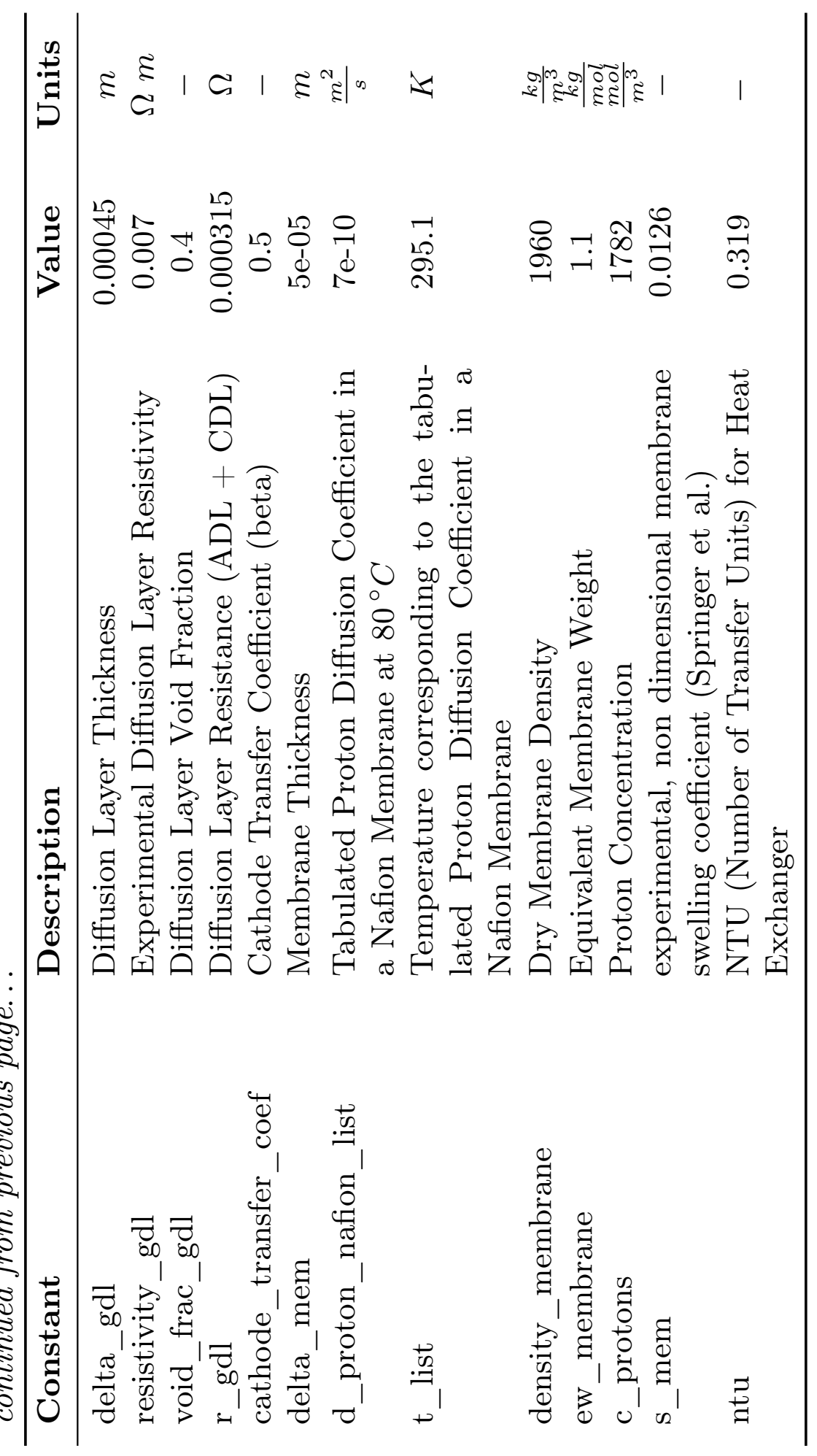





\section{List of Figures}

1.1. Schematic representation of different energy (battery, fuel cell and internal combustion engine) converters in the form of black-boxes. . . . . . . . . . . . . . 3

1.2. Typical Internal Combustion Engine and Fuel Cell efficiencies as a function of engine load. The bar-graph depicts the percentage at which the corresponding engine load occurs in the European ECE-15 driving cycle. Data from [4]. . . . 4

1.3. Schematic representation of a PEM fuel cell. The drawing is not to scale, but typical dimensions have been indicated on the drawing. . . . . . . . . . . . . . . 7

2.1. Chemical structure of Nafion. The long main chain is the base PTFE material upon which the carrying $\mathrm{SO}_{3}^{-}$side chains have been grafted. . . . . . . . . . . . . . . 27

2.2. Membrane water content as a function of water activity for Nafion 117. . . . . . . . . . . . . . . . .

2.3. Membrane conductivity dependence on membrane water content based on Springer et al. (2.20), on Mann et al. (2.21) and on a theoretical Schlögl/Nernst approach (A.33) (parameter values for (A.33) are taken from Bernardi et al. (see Table A.1)). . . . . . . . . . . . . . . 30

2.4. Schematic representation of species transport within a PEM fuel cell repeat element. Nitrogen flow is not represented on the cathode side since it does not participate in the electrochemical reactions. . . . . . . . . . . . 32

2.5. Molar inlet and outlet flow rates for the anode gas channel. 34

2.6. Molar inlet and outlet flow rates for the cathode gas channel. 34 
2.7. Molar water fraction in a gas mixture as a function of relative humidity $\psi$, gas pressure and temperature. See comments on page 35 for the motivation behind running fuel cells at higher gas pressures. . . . . . . . . . . . . 35

2.8. Schematic representation of the reaction gas transport in the z-direction perpendicular to the membrane, shown here for the case of oxygen. The same mass transport mechanisms are responsible for hydrogen transport in the anode compartment. 40

2.9. Water transport across the GDL-membrane-GDL sandwich within a fuel cell. . . . . . . . . . . . . . . . . . 44

2.10. Average molar water fraction in the cathode gas channel for varying operating parameters. The blueish, upper horizontal surface represents the saturation level while the grayish, lower horizontal surface shows the minimum average water content. . . . . . . . . . . . . . . . 46

2.11. Average molar water fraction in the anode gas channel for varying operating parameters. The blueish, upper horizontal surface represents the saturation level while the grayish, lower horizontal surface shows the minimum average water content. . . . . . . . . . . . . .

2.12. Maximum and minimum water transfer coefficients depending on the inlet molar water fraction and the stoichiometric flow rate in the cathode and anode GC respectively. . . . .

2.13. Water diffusivity in the membrane region at $30^{\circ} \mathrm{C}$ as a function of membrane water content (Experimental values as determined by [12] and interpolated values according to (2.91) to $(2.95)) . \ldots \ldots \ldots \ldots \ldots$

2.14. Overview of the different contributions (drag, diffusive and convective) to water transport across the membrane. The water flux rates $\left[\frac{m o l}{s \cdot m^{2}}\right]$ have been calculated for standard literature values for the parameters and are shown as a function of the driving force(s). For the case of diffusive transport, the flux rate is also shown as a function of the water diffusivity in the membrane since there are large differences between the values proposed in literature. . . . . . . . . .

2.15. Flowchart outlining the fuel cell stack model elaborated in chapter 2. . . . . . . . . . . . . . . 
3.1. Polymer Electrolyte Fuel Cell System. . . . . . . . . . . . 65

3.2. Model schematic for the air supply subsystem. . . . . . . 66

3.3. Schematic of the air compressor. . . . . . . . . . . 67

3.4. Schematic of the backpressure regulator. . . . . . . . . . 71

3.5. Experimental backpressure regulation valve characteristics. The lines for a valve voltage ${ }^{1}$ of 1,2 and (partially) $3 \mathrm{~V}$ (large valve openings) correspond to the model - at smaller valve openings, the valve characteristics are inverted. . . . . 72

3.6. Model schematic for the hydrogen supply subsystem. . . . . 73

3.7. Schematic drawing of a spring loaded pressure regulator with one sensing volume. . . . . . . . . . . . . 75

3.8. Pressure evolution in the hydrogen supply manifold following a sudden opening of a discharge valve. The results are shown with and without pressure regulator. . . . . . . . 76

3.9. Model schematic for the thermal management subsystem. . 77

3.10. Heat transfer through free convection along the fuel cell that is considered as a vertical flat plate. . . . . . . . . . 81

3.11. Heat exchanger efficiency $\epsilon$ for various hot and cold water flow rate conditions. . . . . . . . . . . . 83

4.1. Picture of the BRESA FC stack installed at LEI. . . . . . 97

4.2. IV curve of the stack's first test run with the operating conditions $T_{\text {stack }}=60^{\circ} \mathrm{C}, T_{\text {air }}=70^{\circ} \mathrm{C}$, air dew-point $T_{D P}=60^{\circ} \mathrm{C}$ and non-controlled stoichiometries. . . . . . 98

4.3. Description of the experimental system. . . . . . . . . . 99

4.4. Picture of the fuel cell test stand installed at LEI. . . . . . 100

4.5. Characteristics of the installed ASF Thomas 2907CDC22 double head membrane compressor (manufacturer data). . . 101

4.6. Schematic representation of the Arbin DHP humidification system. . . . . . . . . . . . . . . . 103

4.7. Experimental temperature evolution of the dew point $\left(T_{D P}\right)$ and exit heater $\left(T_{E H}\right)$ temperatures. . . . . . . . . 103

4.8. Characteristics of the Air Liquide HBS 315/1 spring loaded pressure regulator (manufacturer supplied data). . . . . . 104

4.9. Characteristics of the installed KNF $N 828 K N D C$ single head membrane pump (manufacturer data) . . . . . . . 106

4.10. Characteristics of the installed KNF N 300 KPDC single head membrane pump (manufacturer data). . . . . . . . 107 
4.11. Characteristics of the Gardena 4000/4i water pump (manufacturer data). . . . . . . . . . . . . . . 108

4.12. User interface for the data acquisition and control system. . 118

5.1. Schematic representation of the fuel cell stack model. . . . . 122

5.2. Performance evolution of the BRESA fuel cell stack installed at $L E I$. For reference purposes, the current-voltage characteristic [56] of a new stack running under design operating conditions is also shown. Note that the reference system is operated at a pressure of $2 \operatorname{bar}(a)$ whereas the LEI system runs at ambient pressure. . . . . . . . . . . . . . . 123

5.3. Comparison between experimental and modelled fuel cell voltage. The experimental data [56] was recorded at an average cell temperature of $T_{F C}=70^{\circ} \mathrm{C}$, stoichiometric flow rates of $\lambda_{\text {air }}=\lambda_{H_{2}}=2$ and dew point temperatures of $T_{D P}=50^{\circ} \mathrm{C}$ and $T_{D P}=65{ }^{\circ} \mathrm{C}$ respectively. . . . . . . 125

5.4. Modelled water transfer coefficient $\alpha$ and partial reactant pressures for the performance data shown in Figure 5.3. . . 126

5.5. Experimental and modelled water transfer coefficients $\alpha$ used for identification of the water transport relevant model parameters. . . . . . . . . . . . . . . . 130

5.6. Experimental and modelled average cell voltage for varying operating conditions. Note that because of the unstable behaviour of cell 7 (noted $V_{7}$ in the legend), the modelled data was compared to the average cell voltage for cells 2-6. Under each figure are indicated: The fuel cell temperature, the air and hydrogen dew point temperatures along with the air and hydrogen flow rate stoichiometries. . . . . . . 133

5.7. Identification of the air compressor motor's torque constant $k_{t} \ldots \ldots \ldots \ldots \ldots \ldots \ldots \ldots \ldots$

5.8. Identification of the air compressor's speed-mass flow coefficient $k_{m} \ldots \ldots \ldots \ldots \ldots \ldots$. . . . . . . . 137

5.9. Experimental data used for parameter identification of the air supply subsystem. The fuel cell was simulated in the experimental set-up through a valve with a similar flow resistance which was varied during the experiment. The model corresponds to the one outlined in 3.3.1 where the air inlet pressure is considered a system input. 
5.10. Pressure drop across the fuel cell cathode gas channel. For reference purposes, the design characteristic [34] of the stack is also shown. . . . . . . . . . . . . . . . . 139

5.11. Experimental results compared to modelled data for the air supply subsystem. The fuel cell was simulated in the experimental set-up through a valve with a similar flow resistance. . . . . . . . . . . . . . . . . 140

5.12. Pressure drop across the anode compartment of the fuel cell stack. . . . . . . . . . . . . . . . . . 143

5.13. Experimental results used for identification of the hydrogen supply subsystem model. . . . . . . . . . . . . . . . . . 144

5.14. Experimental results used for the verification of the hydrogen supply subsystem model. . . . . . . . . . . . . . . . 145

5.15. Experimental demonstration of the dynamics of the pressure regulation system in the hydrogen supply manifold. . . . . . 147

5.16. Identification of the cooling liquid pump motor's torque constant $k_{t, C P}$ and speed-mass flow coefficient $k_{m, C P} \ldots 149$

5.17. Experimental temperature evolution within the cooling circuit when subjected to a constant heating power of $200 \mathrm{~W}$. The cooling liquid flow rate is $\dot{m}_{C L}=1.1 \mathrm{lpm}$. The corresponding heat transferred from the CL is shown in Figure 5.19.151

5.18. Temperature evolution of the CL reservoir when cooling from an initial temperature with no cooling liquid flow. . . 153

5.19. Heat transfer from the cooling liquid when subjected to a constant heating power of $200 \mathrm{~W}$. The cooling liquid flow rate is $\dot{m}_{C L}=1.1 \mathrm{lpm}$. The corresponding temperature evolution is shown in Figure 5.17. . . . . . . . . . . . . 154

5.20. Experimental results used for the identification of the heat exchanger parameters. . . . . . . . . . . . . 155

5.21. Experimental results used for the validation of the cooling liquid pump model. . . . . . . . . . . . . . . . 157

5.22. Experimental results used for the validation of the heat exchanger parameters. . . . . . . . . . . . . 158

A.1. Schematic representation of the limiting current density on a graph showing the reactant concentration $c_{i}$ as a function of the distance across the gas diffusion layer. . . . . . . 176 
B.1. Henry's constant as a function of temperature. . . . . . . 183

B.2. Oxygen Diffusivity - Pressure product $p \cdot D_{O_{2} L}$ for oxygen diffusion in humid air as a function of inlet water molar fraction, water transfer coefficient and stoichiometric air ratio at a temperature of $70^{\circ} \mathrm{C} \ldots \ldots \ldots \ldots$ 


\section{List of Tables}

1.1. Comparison of different fuel cells and their operating characteristics. Data from $[6] . \ldots \ldots$. . . . . . . . .

2.1. FC model inputs and FC operating parameters. . . . . . . . 18

2.2. Molar reaction enthalpies and equilibrium voltage $E_{00}$ for the fuel cell reaction $\mathrm{H}_{2}+\frac{1}{2} \mathrm{O}_{2} \rightarrow \mathrm{H}_{2} \mathrm{O}$ for standard reaction conditions (i.e. $T_{0}=25^{\circ} \mathrm{C}$ and $p_{0}=101^{\prime} 325 \mathrm{~Pa}$ ). Data from [33]. . . . . . . . . . . . . . . . . . . . 19

2.3. Typical exchange current densities for PEFCs (Data from [35]) . . . . . . . . . . . . . . .

2.4. Coefficients for the general expression of the water diffusion coefficient in the membrane phase according to different experimental data and curve fits. . . . . . . . . . . . 52

2.5. Typical literature values for the hydraulic membrane permeability $k_{p} \ldots \ldots \ldots \ldots \ldots \ldots$

2.6. Operation condition dependency matrix for the fuel cell stack model. - denotes a direct dependence of the variable on the considered operating condition. 0 denotes an indirect dependence where the variable depends on another, previously calculated variable that is itself dependent on the

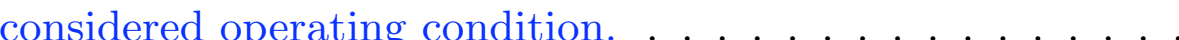

5.1. Parameter dependency matrix for the fuel cell stack model. - denotes a direct dependence of the variable on the considered parameter. $\bigcirc$ denotes an indirect dependence where the variable depends on another, previously calculated variable that is itself dependent on the considered parameter.129

5.2. Literature fuel cell stack model parameters compared to values identified for experimental data from Büchi et al. [56] and experimental data from the LEI test bench. . . . . . . 132 
5.3. Experimentally identified air supply subsystem parameters. 141

5.4. Experimentally identified hydrogen supply subsystem parameters. . . . . . . . . . . . . . . 146

5.5. Experimentally identified cooling liquid pump parameters. . 150

5.6. Experimentally identified bipolar plate parameters. . . . . . 152

5.7. Experimentally identified cooling liquid reservoir parameters. 153

5.8. Experimentally identified cooling liquid reservoir parameters. 154

A.1. Values reported in literature for ionic membrane conductivity, proton diffusion coefficient and electrokinetic permeability. 180

B.1. Partial pressures and molar content of air at standard conditions (i.e. $T_{0}=25^{\circ} \mathrm{C}$ and $p_{0}=101^{\prime} 325 \mathrm{~Pa}$ ). . . . . 182 


\section{Bibliography}

[1] A. Wokaun, U. Baltensprerger, K. Boulochos, F. Gassmann, W. Hoffelner, P. Jansohn, R. Palumbo, G. Scherer, A. Steinfeld, and S. Stucki, "The role of hydrogen in a future sustainable energy system - under which circumstances does a hydrogen economy make sense?," Executive summary of a seminar series held at PSI during the first semester 2004, PSI and ETH, 2004.

[2] U. Bossel, B. Eliasson, and G. Taylor, Final Report: The Future of the Hydrogen Economy: Bright or Bleak? www.efcf.com, April 2003.

[3] R. Shinnar, "The hydrogen economy, fuel cells, and electric cars," Technology in Society, vol. 25, no. 4, pp. 455-476, 2003.

[4] G. Pede, A. Iacobazzi, S. Passerini, A. Bobbio, and G. Botto, "FC vehicle hybridisation: An affordable solution for an energy-efficient FC powered drive train," Journal of Power Sources, vol. 125, pp. 280 291, 2004.

[5] W. Grove, "On the gas voltaic battery - voltaic action of phosphorus, sulphur and hydrocarbons," Phil. Transactions, vol. 1, p. 351, 1845.

[6] G. Hoogers, ed., Fuel Cell Technology Handbook. CRC Press, 2003.

[7] U. Pasaogullari and C.-Y. Wang, "Two-phase modeling and flooding prediction of polymer electrolyte fuel cells," J. Electrochem. Soc., vol. 152, no. 2, pp. A380-A390, 2005.

[8] C. Rivas, Etude, modélisation et propriétés d'un onduleur de courant à pulsation associé à une pile à combustible. $\mathrm{PhD}$ thesis, Ecole Polytechnique Fédérale de Lausanne, 2003.

[9] T. Berning, D. M. Lu, and N. Djilali, "Three-dimensional computational analysis of transport phenomena in a pem fuel cell," Journal of Power Sources, vol. 106, no. 1-2, pp. 284-294, 2002. 
[10] N. P. Siegel, M. W. Ellis, D. J. Nelson, and M. R. von Spakovsky, "A two-dimensional computational model of a PEMFC with liquid water transport," Journal of Power Sources, vol. 128, no. 2, pp. 173-184, 2004 .

[11] S. Freunberger, A. Tsukada, G. Fafilek, and F. Büchi, "1+1 dimensional model of a PE fuel cell of technical size," PSI Scientific Report 2002, vol. V, pp. $94-95,2003$.

[12] T. Springer, T. Zawodzinski, and S. Gottesfeld, "Polymer electrolyte fuel cell model," J. Electrochem. Soc., vol. 138, no. 8, pp. $2334-2342$, 1991.

[13] D. Bernardi and M. Verbrugge, "A mathematical model of the solidpolymer-electrolyte fuel cell," J. Electrochem. Soc., vol. 139, no. 9, pp. $2477-2491,1992$.

[14] T. Nguyen and R. White, "A water and heat management model for proton-exchange-membrane fuel cells," J. Electrochem. Soc., vol. 140, no. 8, pp. $2178-2186,1993$.

[15] T. Fuller and J. Newman, "Water and thermal management in solidpolymer-electrolyte fuel cells," J. Electrochem. Soc., vol. 140, no. 5, pp. $1218-1225,1993$.

[16] C. Marr and X. Li, "Composition and performance optimization of catalyst layer in a proton exchange membrane fuel cell," Proceedings of the ASME Asia Congress and Exhibition, 1997.

[17] A. Rowe and X. Li, "Mathematical modeling of proton exchange membrane fuel cells," Journal of Power Sources, vol. 102, pp. 82 - 96, 2001.

[18] V. Gurau, F. Barbir, and H. Liu, "Two-dimensional model for the entire sandwich of a PEM fuel cell," Electrochemical Society Proceedings, vol. 98 - 27, pp. $479-503,1999$.

[19] S. Dutta, S. Shimpalee, and J. V. Zee, "Three-dimensional numerical simulation of straight channel pem fuel cells," Journal of Applied Electrochemistry, vol. 30, pp. 135 - 146, 2000. 
[20] S. Um and C. Y. Wang, "Three-dimensional analysis of transport and electrochemical reactions in polymer electrolyte fuel cells," Journal of Power Sources, vol. 125, no. 1, pp. 40-51, 2004.

[21] B. R. Sivertsen and N. Djilali, "CFD-based modelling of proton exchange membrane fuel cells," Journal of Power Sources, vol. 141, no. 1, pp. 65-78, 2005.

[22] J. Amphlett, R. Mann, B. Peppley, P. Roberge, and A. Rodrigues, "A practical PEM fuel cell model for simulating vehicle power sources," unknown, pp. 221 - 226, 1995.

[23] J. Amphlett, R. Baumert, R. Mann, B. Peppley, P.R.Roberge, and T. Harris, "Performance modeling of the Ballard MarkIV solid polymer electrolyte fuel cell," J. Electrochem. Soc., vol. 142, no. 1, pp. 1 - 8, 1995. I. Mechanistic Model Development.

[24] J. Kim, S. Lee, and S.Srinivasan, "Modeling of proton exchange membrane fuel cell performance with an empirical equation," J. Electrochem. Soc., vol. 142, no. 8, pp. 2670 - 2674, 1995.

[25] J. H. Lee, T. R. Lalk, and A. J. Appleby, "Modeling electrochemical performance in large scale proton exchange membrane fuel cell stacks," Journal of Power Sources, vol. 70, pp. 258-268, 1998.

[26] R. Mann, J. Amphlett, M. Hooper, H. Jensen, B. Peppley, and P. Roberge, "Development and application of a generalised steadystate electrochemical model for a PEM fuel cell," J. of Power Sources, vol. 86, pp. $173-180,2000$.

[27] P. H. Rodatz, Dynamics of the Polymer Electrolyte Fuel Cell: Experiments and Model-Based Analysis. PhD thesis, Eidgenössische Technische Hochschule Zürich, 2004.

[28] F. Grasser and A. Rufer, "A fully analytical PEM fuel cell system model for control applications," in Proceedings of the IEEE Industry Applications Society Meeting, Submitted to the editor, (Tampa, Florida), 2006.

[29] J. Pukrushpan, A. Stefanopoulou, and H. Peng, Control of Fuel Cell Power Systems. Advances in Industrial Control, London: Springer, 1 ed., 2004. 
[30] A. Miotti, A. D. Domenico, Y. Guezennec, and S. Rajagopalan, "Control-oriented model for an automotive PEM fuel cell system with imbedded 1+1D membrane water transport," in Proceedings of the 2005 IEEE Vehicle Power and Propulsion Conference, pp. 611-618, 2005 .

[31] A. Hernandez, D. Hissel, and R. Outbib, "Non linear state space modelling of a pemfc," in Proceedings of the 2nd France-Deutschland Fuel Cell Conference, pp. 433-438, 2004.

[32] F. Grasser and A. Rufer, "PEM fuel cell system efficiency optimisation through model based control strategies," To be published, 2006.

[33] J. V. Herle and A. Rufer, Electrochemical Technology, Fuel Cells and Batteries. Lausanne, Switzerland: EPFL, 2002.

[34] M. Ruge, Entwicklung Eines Flüssigkeitsgekühlten Polymer-ElektrolytMembran-Brennstoffzellenstapels mit Einer Leistung Von $6.5 \mathrm{~kW}$. $\mathrm{PhD}$ thesis, ETHZ, 2003.

[35] B. Andreaus, Die Polymer-Elektrolyt Brennstoffzelle: Charakterisierung Ausgewählter Phänomene Durch Elektrochemische Impedanzspektroskopie. PhD thesis, Ecole Polytechnique Fédérale de Lausanne, 2002.

[36] A. Parthasarathy, S. Srinivasan, A. Appleby, and C. Martin, "Temperature dependence of the electrode kinetics of oxygen reduction at the Platinum/Nafion interface - a microelectrode investigation," $J$. Electrochem. Soc., vol. 139, pp. 2530 - 2537, 1992.

[37] D. Bernardi and M. Verbrugge, "Mathematical model of a gas diffusion electrode bonded to a polymer electrolyte," AIChE Journal, vol. 37, pp. 1151 - 1163, August 1991.

[38] P. v. Schroeder, "Ueber erstarrungs- und quellungserscheinungen von gelatine," Journal of Power Sources, vol. 45, no. 2, p. 75, 1903.

[39] J. Hinatsu, M. Mizuhata, and H. Takenaka, "Water uptake of perfluorosulfonic acid membranes from liquid water and water vapor," Journal of the Electrochemical Society, vol. 141, pp. 1493 - 1497, 1994. 
[40] F. Büchi and G. Scherer, "In-situ resistance measurements of nafion 117 membranes in polymer electrolyte fuel cells," Journal of Electroanalytical Chemistry, vol. 404, pp. 37 - 43, 1996.

[41] T. Springer, T. Zawodzinski, M. Wilson, and S. Gottesfeld, "Characterization of polymer electrolyte fuel cells using AC impedance spectroscopy," J. Electrochem. Soc., vol. 143, no. 2, pp. 587 - 599, 1996.

[42] P. Rieke and N. Vanderborgh, "Temperature dependence of water content and proton conductivity in polyperfluorosulfonic acid membranes," Journal of Membrane Science, vol. 32, pp. 313 - 328, 1987.

[43] L. Gerbaux, Modélisation D'une Pile À Combustible de Type Hydrogene/Air et Validation Experimentale. PhD thesis, Institut National Polytechnique de Grenoble, 1996.

[44] D. Nield and A. Bejan, Convection in Porous Media. New York: Springer Verlag, 1992. BC: DA808.

[45] P. Berg, K. Promislow, J. S. Pierre, J. Stumper, and B. Wetton, "Water management in pem fuel cells," Journal of The Electrochemical Society, vol. 151, 2004.

[46] S. Motupally, A. Becker, and J. Weidner, "Diffusion of water in Nafion 115 membranes," Journal of the Electrochemical Society, vol. 147, no. 9, pp. $3171-3177,2000$.

[47] T. Zawodzinski, M. Neeman, L. Sillerud, and S. Gottesfeld, "Determination of water diffusion coefficients in perfluorosulfonat ionomeric membranes," Journal of Physical Chemistry, vol. 95, pp. 6040 - 6044, 1991.

[48] B. Olsommer, "Mathematical model for a proton exchange membrane fuel cell," tech. rep., Virginia Tech, 1999.

[49] J. Fales and N. Vanderborgh, "The influence of ionomer channel geometry on ionic transport," Proceedings of the Symposium on Diaphragms, Separators, and Ion-Exchange Membranes, vol. 86-13, pp. 179 - 191, 1986. 
[50] P. Dietrich, F. Büchi, A. Tsukada, M. Bärtschi, R. Kötz, G. Scherer, P. Rodatz, O. Garcia, M. Ruge, M. Wollenberg, P. Lück, A. Wiartalla, C. Schönfelder, A. Schneuwly, and P. Barrade, Hy.Power - A Technology Platform Combining a Fuel Cell System and a Supercapacitor, vol. 4, ch. 11, pp. 1184 - 1198. John Wiley \& Sons, Ltd, 2003.

[51] D. Schmid, Entwicklung eines Brennstoffzellenstapels für portable Aggregate unterschiedlicher Leistungsbereiche. PhD thesis, ETHZ, 2003.

[52] B. Munson, D. Young, and T. Okiishi, Fundamentals of Fluid Mechanics. New York: John Wiley \& Sons, 3 ed., 1998.

[53] A. Iberall, "Static-flow characteristics of single and two-stage springloaded gas-pressure regulators," Transactions of the ASME, vol. 76, p. 363, 1954.

[54] D. Tsai and E. Cassidy, "Dynamic behavior of simple pneumatic pressure reducer," ASME J. Basic Eng., vol. 83, pp. 253-264, 1961.

[55] S. Freunberger and F. Büchi, "Model approach for the description of lateral effects in large scale PEFC," in Proceedings of the Fuel Cell Research Symposium, (Zürich), March 2004.

[56] F. Büchi and M. Ruge, "Development of a bipolar element for PE fuel cells demonstration in a $6 \mathrm{~kW}$ stack," PSI Scientific Report 2000 (General Energy), vol. V, pp. 84-85, 2001.

[57] F. Incropera and D. DeWitt, Introduction to Heat Transfer. New York: John Wiley and Sons, 1996.

[58] W. Kays and M. Crawford, Convection Heat and Mass Transfer. New York: McGraw-Hill, 1980.

[59] R. Gemmen, "Analysis for the effect of inverter ripple current on fuel cell operating condition," Journal of Fluids Engineering, vol. 125, no. 3, pp. 576-585, 2003.

[60] F. Büchi, P. Rodatz, A. Tsukada, and P. Dietrich, "Fuel cell system for the HY.POWER hybrid electric vehicle," PSI Scientific Report 2002, vol. V, pp. $102-103,2003$. 
[61] F. de Bruijn, "The current status of fuel cell technology for mobile and stationary applications," Green Chemistry, vol. 7, pp. 132-150, 2005.

[62] A. Panchenko, H. Dilger, J. Kerres, M. Hein, A. Ullrich, T. Kaz, and E. Roduner, "In-situ spin trap electron paramagnetic resonance study of fuel cell processes," Phys. Chem. Chem. Phys., vol. 6, no. 2891-2894, 2004 .

[63] B. Mattsson, H. Ericson, L. M. Torell, and F. Sundholm, "Degradation of a fuel cell membrane, as revealed by micro-raman spectroscopy," Electrochimica Acta, vol. 45, no. 8-9, pp. 1405-1408, 2000.

[64] S. D. Knights, K. M. Colbow, J. St-Pierre, and D. P. Wilkinson, "Aging mechanisms and lifetime of PEFC and DMFC," Journal of Power Sources, vol. 127, no. 1-2, pp. 127-134, 2004.

[65] F. Opekar and D. Svozil, "Electric resistance in a Nafion membrane exposed to air after a step change in the relative humitity," Journal of Electroanalytical Chemistry, vol. 385, pp. 269 - 271, 1995.

[66] M. Verbrugge and R. Hill, "Analyisis of promising perfluorosulfonic acid membranes for fuel-cell electrolytes," Journal of the Electrochemical Society, vol. 137, pp. 3770 - 3777, December 1990.

[67] M. Verbrugge and R. Hill, "Transport phenomena in perfluorosulfonic acid membranes during the passage of current," Journal of the Electrochemical Society, vol. 137, pp. 1131 - 1138, April 1990.

[68] M. Verbrugge and R. Hill, "Ion and solvent transport in ion-exchange membranes," Journal of the Electrochemical Society, vol. 137, pp. 886 - 899, March 1990.

[69] L. Borel, Thermodynamique et Energétique, vol. I. Lausanne: PPUR, 3ème ed., 1991.

[70] R. Perry and D. Green, Perry's Chemical Engineers' Handbook. Singapore: McGraw-Hill Book Co., 6th ed., 1984.

[71] R. Battino, "The solubility of gases in liquids," Chemical Reviews, vol. 66, p. 395, 1966. 
[72] C. Bennet and J. Myers, Momentum, Heat, and Mass Transfer. Singapore: McGraw-Hill Book Co., 3rd ed., 1983.

[73] E. W. Weisstein, "Cubic formula."

[74] D. Gillet, Commande avancée II: Systèmes multivariables. Lausanne: EPFL, 1998. 
Curriculum Vitae 


\title{
Education \& Experience
}

\author{
2000 to date Research assistant \& PhD student \\ Laboratoire d'Electronique Industrielle, Ecole \\ Polytechnique Fédérale de Lausanne, Lausanne, \\ Switzerland \\ 1998 - $2000 \quad$ MS Mechanical Engineering \\ Ecole Polytechnique Fédérale de Lausanne, Lausanne, \\ Switzerland \\ 1995 - 1998 BS Mechanical Engineering \\ Ecole Polytechnique Fédérale de Lausanne, Lausanne, \\ Switzerland and Virginia Polytechnic Institute and State \\ University, Blacksburg, USA \\ $1994-1995 \quad$ Corrugated cardboard designer \\ Corrugated Products Ltd., Bracknell, UK \\ $1987-1994 \quad$ High school \\ Kantonsschule, Sursee, Switzerland
}

Languages

German Native language

English Fluent (spoken and written)

French Fluent (spoken and written)

\section{Selected Publications}

F. Grasser and A. Rufer. Advanced fuel cell system control through state space modeling. In Proceedings of IFAC 2005: 16th International Federation of Automatic Control World Congress, Prag, July 2005.

F. Grasser and A. Rufer. A simple PEFC system model for control system development. In Proceedings of the France Deutschland Fuel Cell Conference, Belfort, December 2004. 
F. Grasser and A. Rufer. Advances in fuel cell modeling for control system development. In Proceedings of the Fuel Cell Reserach Symposium on Modelling and Experimental Validation, Zürich, 2004.

S. Lemofouet, A. Rufer, I. Cyphelly, P. Barrade, and F. Grasser. Principle of a hybrid compressed air and supercapacitors energy storage system with maximum efficiency point tracking. In Proceedings of STORE: Storage for Renewable Energies, Aix-en-Provence, France, October 2003.

S. Lemofouet, A. Rufer, I. Cyphelly, P. Barrade, and F. Grasser. Principle of a hybrid compressed air and supercapacitors energy storage system with maximum efficiency point tracking. In Proceedings of EESAT: Electric Energy Storage Applications 83 Technologies, San Francisco, USA, October 2003.

F. Grasser and A. Rufer. Fuel cell system modeling for control system development. In Proceedings of the 1st Swiss Fuel Cell Seminar, Yverdon, 2003.

F. Grasser, A. D'Arrigo, S. Colombi, and A. Rufer. JOE: A mobile, inverted pendulum. IEEE Transactions on Industrial Electronics, 49:107-114, 2002.

F. Grasser, A. D'Arrigo, S. Colombi and A. Rufer. JOE: A mobile, inverted pendulum. In Proceedings of the IASTED International Conference Modelling, Identification, and Control, Innsbruck, Austria, 2001. 